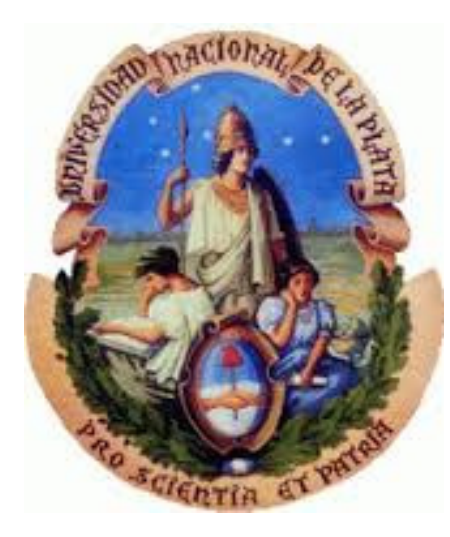

\title{
Caracterización de guías de onda ópticas generadas con láser de femtosegundos en Niobato de Litio
}

\section{Matías Rubén Tejerina}

\author{
Universidad Nacional de La Plata
}

Facultad de Ingeniería, Departamento de Mecánica

La Plata, Argentina

2014 



\title{
Caracterización de guías de onda ópticas generadas con láser de femtosegundos en Niobato de Litio \\ Matías Rubén Tejerina
}

\author{
Tesis doctoral presentada \\ ante la \\ Facultad de Ingeniería de la \\ Universidad Nacional de La Plata \\ para acceder al grado de: \\ DOCTOR EN INGENIERIA
}

Director:

Dr. Gustavo Adrián Torchia

Co-director:

Dr. Gabriel Mario Bilmes

Línea de Investigación:

Micro-estructuración de Materiales para Fotónica Integrada

Centro de Investigaciones Ópticas de La Plata (CONICET-CIC)

La Plata, Argentina

2014 

"...la obsesión por los métodos cuantitativos encubre, en la ilusión de la libertad de investigación, un mecanismo que garantiza la sujeción del científico a las estrategias de expansión del capital y las leyes del mercado." O. Varsavsky (Ciencia, Política y Cientificismo, Buenos Aires: CEAL, 1969). 



\section{Resumen}

En esta tesis se estudian algunos aspectos de la interacción de láser de femto-segundos con cristales de niobato de litio aplicada a la fabricación de guías de onda. Se analizan los campos de deformaciones residuales de la interacción que permiten generar estas guías de onda. Los principales métodos experimentales de caracterización utilizados en este análisis son: mapeos espectroscópicos $\mu$-Raman y medidas de campo cercano de modos guiados. Las medidas experimentales se ajustaron y/ó compararon con resultados de un modelo numérico que representa una expansión arbitraria de una elipse en el seno de un material elástico. A partir de este proceso, fue posible encontrar interesantes resultados relacionados con las características de la expansión producida por la interacción.

El modelo numérico utilizado se generó en entorno MATLAB y resuelve tanto el problema elástico bidimensional para una expansión arbitraria, como así también, los modos guiados originados por su deformación residual.

A su vez, se presentó y aplicó un método denominado "directo" que permite obtener el campo de índice de refracción aproximado a partir de mapas $\mu$-Raman, en guías de onda originadas por deformaciones. Este método no requiere un modelo numérico ni iteraciones.

En el presente trabajo de tesis se profundiza sobre el origen de guías de onda fabricadas con láser de femtosegundos, evaluando, a su vez, distintos métodos para estudiar los campos de deformaciones y de índice de refracción. Esto tiene potencial aplicación en el diseño de dispositivos fotónicos, como así también, en el estudio del comportamiento mecánico de materiales ópticos.

Palabras claves: Guías de onda fabricadas con laser de femtosegundos, mapeos micro-Raman, Campo micrométrico de deformaciones residuales. 



\section{Contenido}

Pág.

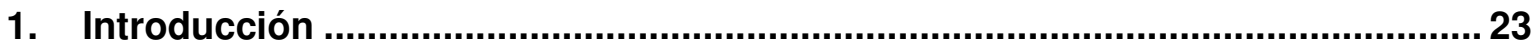

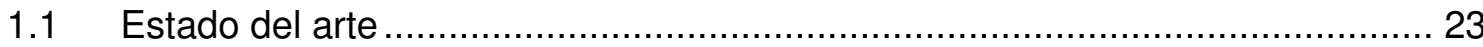

1.1.1 Guías de onda fabricadas con láser de pulsos ultra-cortos ...........................22

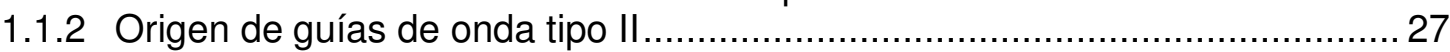

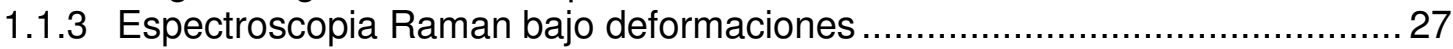

1.1.4 Modelos numéricos aplicados al estudio de guías de onda fabricadas con

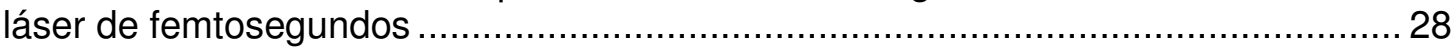
1.1.5 Indentación instrumentada en estructuras fabricadas con láser de

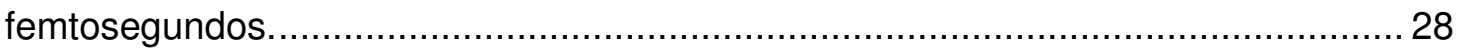

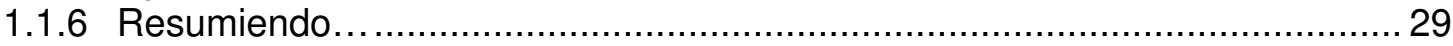

1.2 Interrogantes y problemas a resolver …………….................................... 30

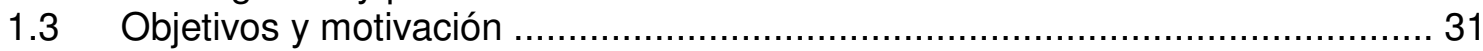

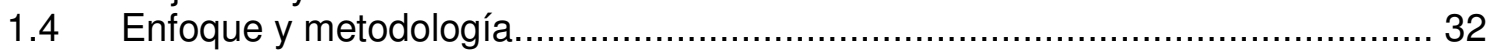

1.5 Estructura y organización de la Tesis ........................................................ 34

2. Marco Teórico: Guías de onda, deformaciones y espectroscopia Raman..........37

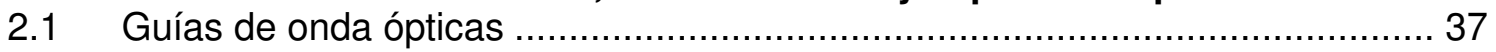

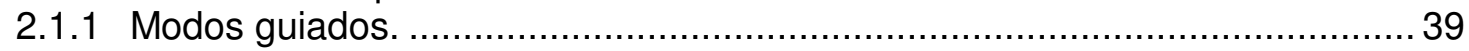

2.1.2 Origen de guías generadas con láser de femtosegundos .......................... 41

2.2 Fenómenos generados durante y después de la interacción ultra-rápida .......... 42

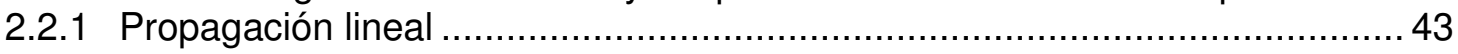

- Aberración esférica y refracción................................................................. 44

2.2.2 Propagación no-lineal. .......................................................................... 45

- Autoenfoque no-lineal y filamentación ..................................................... 46

2.2.3 Absorción de energía................................................................................. 4 4

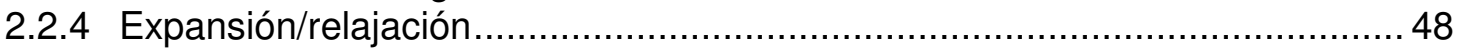

2.3 Deformaciones y tensiones en sólidos deformables. Efecto piezo-óptico .......... 49

2.3.1 Deformaciones y tensiones en sólidos deformables ...................................... 49

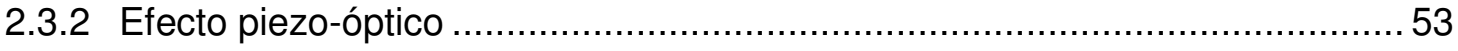

3. Instrumental, técnicas experimentales y materiales empleados ........................55

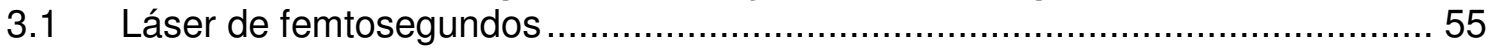

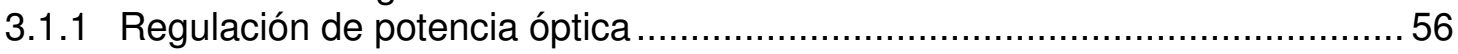

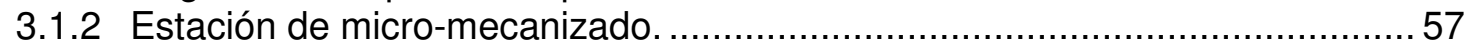

3.2 Acople de luz en guías de onda. ........................................................... 58

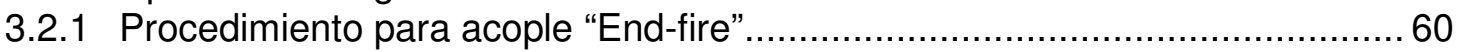

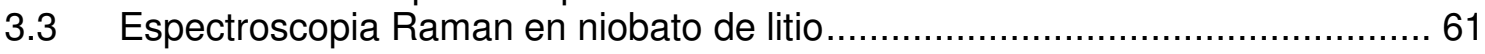

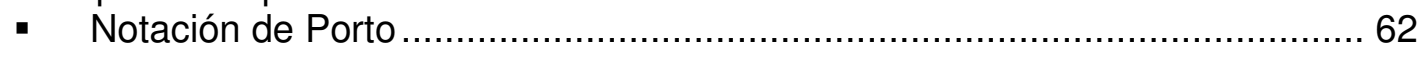

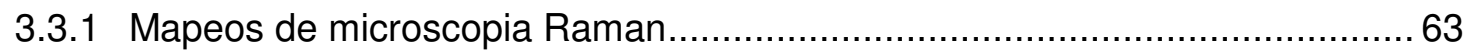

3.4 Espectro Raman de cristales deformados: Teoría de Deformación Potencial

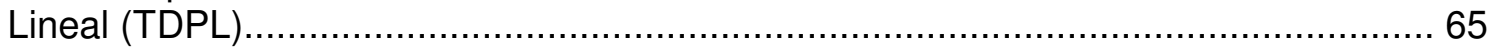

3.5 Sistema de celda de diamantes para compresión hidrostática y sistema de

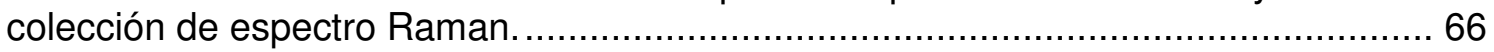

3.5.1 Sistema de aplicación de presiones.......................................................6

3.5.2 Equipamiento Raman para estudio bajo presión hidrostática.........................67

3.5.3 Estudios previos de Raman en niobato de litio bajo presión hidrostática ........67 
X Caracterización de guías de onda ópticas generadas con láser de femtosegundos en Niobato de Litio

3.6 Materiales: niobato de litio puro $\left(\mathrm{LiNbO}_{3}\right)$ y dopado con magnesio y neodimio

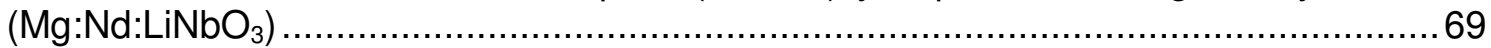

3.7 Preparación de muestras...........................................................................71

3.7.1 Preparación de muestra para ensayo bajo presión hidrostática.......................72

4. Modelos elástico-ópticos de elementos finitos .....................................................75

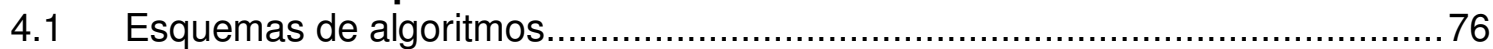

4.2 Método de Elementos Finitos (MEF) para problemas elásticos .........................77

4.2.1 Formulación débil de MEF.....................................................................79

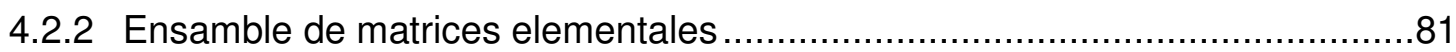

4.2.3 Deformaciones planas: aproximación ortotrópica para niobato de litio. ...........81

4.2.4 Parámetros y condición de simetría.............................................................85

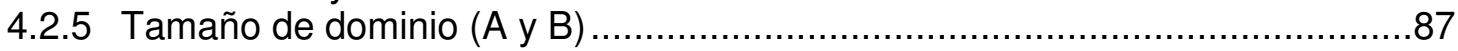

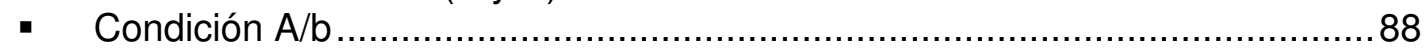

4.2.6 Modelos utilizados y generados en entorno MATLAB .................................90

- Mallado A: elementos cuadriláteros de 9 nodos ............................................91

- Mallado B: elementos triangulares lineales.............................................93

4.2.7 Comparación de modelos en MATLAB con programa comercial ABAQUS ....94

4.3 Cálculo de variación de índice de refracción .....................................................96

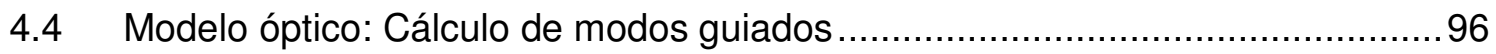

4.4.1 Cálculo de modos guiados en guía plana ...................................................99

4.4.2 Modos guiados en campo de índice de refracción generado por una

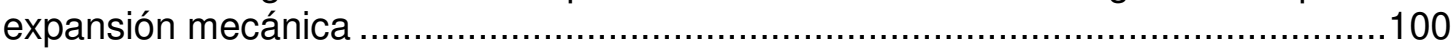

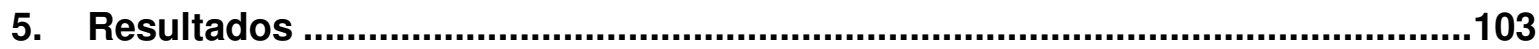

5.1 Estudio $\mu$-Raman bajo presión hidrostática de cristal de $\mathrm{Nd}: \mathrm{Mg}: \mathrm{LiNbO}_{3}$ (niobato de litio dopado con magnesio) ..........................................................................103

5.1.1 Rango completo de presión aplicada (hasta 6.3 GPa) ...............................103

5.1.2 Comportamiento de fonones $A_{1}(T O)$ en rango completo y reducido (hasta 2

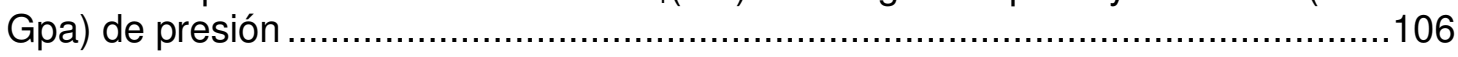

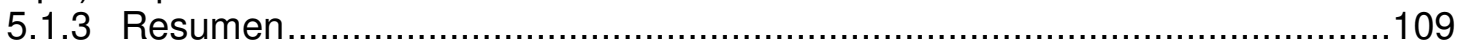

5.2 Estudio micro-Raman y de intensidad guiada de guias de onda fabricadas con laser de femtosegundos .................................................................................110

5.2.1 Guías de onda estudiadas.............................................................110

5.2.2 Análisis unidimensional de guía de onda................................................111

- Resumen de análisis unidimensional....................................................120

5.2.3 Análisis bidimensional de guía de onda (modificación B) ............................120

- Comparación con parámetros calculados en estudio unidimensional...........122

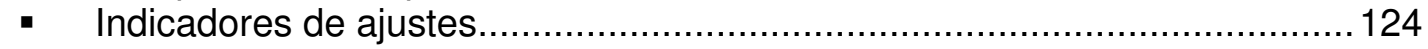

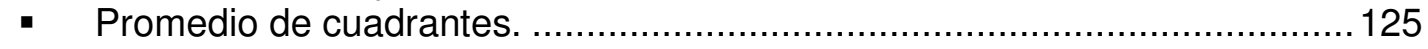

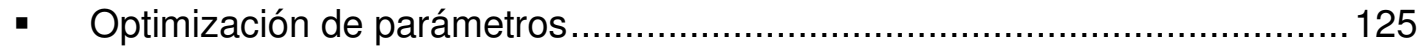

5.3 Cálculo directo de deformaciones, índice de refracción e intensidad guiada

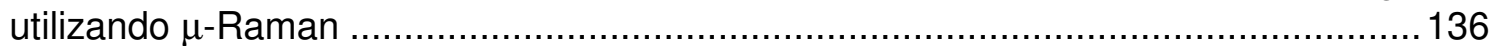

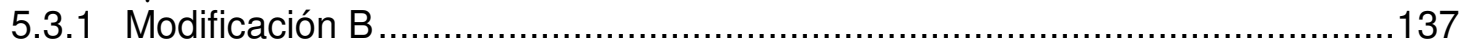

5.3.2 Cálculo directo de modos en guías de onda de doble modificación...............138

5.4 Polarización de luz guiada en modificaciones fabricadas con láser de femtosegundos de diferentes energías de pulso ...................................................143

5.4.1 Comparación cualitativa del modelo numérico con medidas experimentales

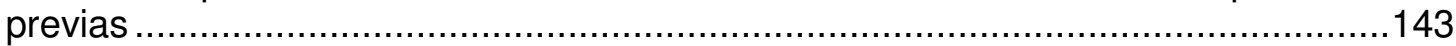

5.4.2 Procedimientos experimentales y resultados............................................145 
6. Conclusiones y trabajo futuro .................................................................. 153

Anexo A: Exploración de guías de onda fabricadas con láser fs utilizando Indentación Instrumentada y $\mu$-Raman ................................................................ 159

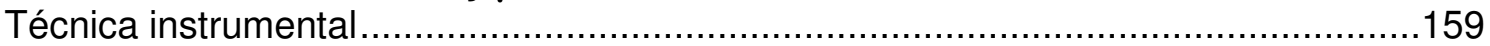

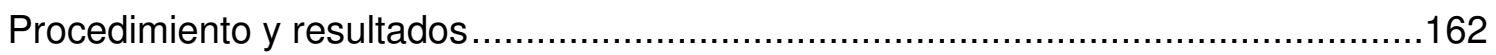

Anexo B: Códigos generados y/ó utilizados para aplicar el Método de Elementos Finitos en entorno MATLAB .................................................................................... 167 



\section{Índice de figuras}

Nombre resumido de Figura / Página

Figura 1. Esquema de guía de onda plana (corte tranversal) / 38

Figura 2. Esquema de guías de onda tridimensionales / 38

Figura 3. Esquema de ondas propagadas en configuración TM y en configuración TE / 39

Figura 4. Guía acanalada que soporta tres modos de propagación / 40

Figura 5. Esquema de enfoque de láser de femto-segundos en material transparente / 42

Figura 6. Corrimiento del foco por la refracción sin aberración. Elongación del volumen focal por aberración esférica / 44

Figura 7. Simulación numérica de propagación no-lineal en el foco en vidrios de fosfato / 46

Figura 8. Esquema del autoenfoque y posterior filamentación de un haz gaussiano / 47

Figura 9.Esquema de autoenfoque y desenfoque / 47

Figura 10. Esquema de tensiones mecánicas en un infinitesimal de sólido / 50

Figura 11.Sistema de generación de láser de femtosegundos / 56

Figura 12. Esquema de sistema láser y estación de micro-mecanizado / 58

Figura 13. Imagen del sistema de micro-mecanizado / 58

Figura 14. Esquema de arreglo experimental para acoplar luz láser a guías de onda / 59

Figura 15. Imagen de sub-sistema acoplador-cristal-objetivo / 60

Figura 16. Imagen de láser acoplado en una guía de onda fabricada con láser de femtosegundos / 61

Figura 17. Esquema de fenómeno de dispersión Raman / 62

Figura 18. Espectro Raman de niobato de litio dopado con magnesio / 63

Figura 19. Esquema de Sistema de Microscopía Raman / 64

Figura 20. Esquema de sistema sistema de compresión de diamantes / 67

Figura 21. Esquema de celda unidad de niobato de litio $\left(\mathrm{LiNbO}_{3}\right) / 70$

Figura 22. Esquema de disposición de sistema crital-vidrios soporte y topes de acero / 72

Figura 23. Diagrama de principales algoritmos utilizados y su vinculación: modelo elástico y modelo óptico / 76

Figura 24. Diagrama de etapas numéricas implementadas en MATLAB / 77

Figura 25. Diferencial de sólido deformable de dos dimensiones / 78

Figura 26. Esquema tridimensional de superposición de volúmenes focales / 82

Figura 27. Mallado tridimensional y mallado bidimensional / 84

Figura 28. Deformaciones para el modelo bidimensional y para el modelo tridimensional sobre la trayectoria b'b" / 84

Figura 29. Esquema de cuarto de dominio / 85 
XIV Caracterización de guías de onda ópticas generadas con láser de femtosegundos en Niobato de Litio

Figura 30. Esquema de parámetros a, b, $\alpha$ y $\beta$ / 86

Figura 31. $K^{\mathrm{d}}$ vs $\varepsilon_{\text {tol }} / 88$

Figura 32. Deformaciones $\varepsilon_{z}$ para distintas expansiones / 89

Figura 33. Mallado de elementos triangulares generado en ABAQUS $®$ / 89

Figura 34. Elemento cuadrado de nueve nodos y elemento triangular de tres nodos / 90

Figura 35. Esquema del mallado $A$ (elementos de nueve nodos) / 92

Figura 36. Resultado de deformaciones $\varepsilon_{\mathrm{zc}}$ utilizando mallado $\mathrm{A} / 92$

Figura 37. Zona central de mallado $B$ para $b / a=2 / 93$

Figura 38. Resultado de deformaciones $\varepsilon_{z c}$ utilizando mallado B / 94

Figura 39. Comparación entre los resultados de los códigos auto-contenidos generados en MATLAB y aquel resultante de utilizar el programa ABAQUS / 95

Figura 40 Mallado de región de guiado y adyacencias en una guía escalón / 97

Figura 41. Cálculo de modos guiados en guía de onda plana / 100

Figura 42. Dominio para cálculo de intensidad guiada / 101

Figura 43. Resultados de expansión mecánica dada por $\alpha=\beta=0.003$ de elipse de radios $a=2 \mu \mathrm{m}$ y $b=9 \mu \mathrm{m} / 102$

Figura 44. (a) Espectro Raman y ajuste de funciones Lorentzianas para la Configuración A / 104

Figura 45. Posición espectral de los fonones Raman medidos en una muestra de $\mathrm{Nd}: \mathrm{Mg}: \mathrm{LiNbO}_{3}$ en función de presión / 106

Figura 46. Posición espectral de fonones $A_{1}(T O)_{i}$ en función de presión hidrostática / 108 Figura 47. Ajuste lineal de la posición de los fonones para rango reducido de presiones (hasta 2 Gpa) / 109

Figura 48. Imágenes microscópicas de la sección transversal de estructuras estudiadas / 111

Figura 49. Esquema de trayectoria b' b" analizada / 112

Figura 50. Espectro Raman en un punto arbitrario de la trayectoria b'b" en configuración $\mathrm{Y}(\mathrm{ZZ}) \mathrm{Y}$ en programa comercial LabSpec® / 112

Figura 51. Ajuste de los corrimientos en número de onda de los fonones $A_{1}(T O)_{1}$ a $A_{1}(\mathrm{TO})_{4}$ a lo largo de la trayectoria $b^{\prime} b$ ", las medidas experimentales se presentan en círculos negros y los ajustes con línea azul / 115

Figura 52. Resultado de ajuste iterativo de los fonones $A_{1}(T O)_{i}$ a lo largo de la trayectoria b'b" $(\mu \mathrm{m}) / 119$

Figura 53. Imagen óptica de modificación estructural analizada / 121

Figura 54. Resultado del modelo numérico con parámetros obtenidos del análisis unidimensional y medidas experimentales / 123

Figura 55. Mapa de corrimiento Raman de fonón $\mathrm{A}_{1}(\mathrm{TO})_{4}$ promediado / 125

Figura 56. Suma de error cuadrático ETC como función de las dimensiones a y b de la elipse / 126

Figura 57. Error Total (ETC) en función de $\beta / \alpha / 128$

Figura 58. Distribuciones de intensidad para distintos parámetros de expansión, (b) Distribución de intensidad experimental / 129

Figura 59. Mapa de corrimiento espectral del fonón $\mathrm{A}_{1} \mathrm{TO}_{4} / 130$ 
Figura 60. Medida experimental de corrimiento Raman en modificación B / 131

Figura 61. ETC vs a vs b para el cuadrante $\mathrm{C}_{2} / 132$

Figura 62. $d \omega / d p$ vs. $\alpha(\beta=0) / 134$

Figura 63. Mapa $\mu$-Raman experimental en cuadrante 2 de fonón $A_{1}(T O)_{1} / 135$

Figura 64. Campos de deformaciones calculados de los mapas Raman de forma directa / 137

Figura 65. Modos de propagación para $\lambda=650 \mathrm{~nm}$ : calculados a partir de los mapas Raman utilizando el método directo / 138

Figura 66. Imagen óptica de guías de modificaciones estructurales del tipo C, modos guiados medidos para ambas polarizaciones / 139

Figura 67. Corrimientos espectrales Raman: fonón $A_{1} T O_{1}$, fonón $A_{1} T O_{2}$ (c) fonón $A_{1} T O_{4}$. $/$ 140

Figura 68. Campo de índice de refracción para los tres pares de fonones: (a), (b) y (c). Modos guiados numéricos para los diferentes pares de fonones / 142

Figura 69. Campo de índice de refracción numérico, componentes $\Delta \mathrm{n}_{\mathrm{xc}}$ y $\Delta \mathrm{n}_{\mathrm{zc}}$ para distintas expansiones representativas / 144

Figura 70. Modos guiados experimentales para diferentes tiempos de pulso en polarización z y polarización x / 144

Figura 71.. Imagen óptica microscópica en transmisión de las estructuras fabricadas con el láser de femto-segundos (canto del cristal). El láser incide desde la parte superior / 146 Figura 72. Esquema experimental para la medición de modos guiados en polarización $\mathrm{x}_{\mathrm{c}}$ / 147

Figura 73. Intensidad guiada de polarización $x_{c} y z_{c}$ en guías generadas a ambos lados de cada modificación estructural (ME) / 149

Figura 74. Modos numéricos y experimentales para ambas polarizciones en los casos 3,4 y $5 / 151$

Figura 75. Campos de índice de refracción numérico $\left(\Delta \mathrm{n}_{\mathrm{xc}}\right.$ y $\left.\Delta \mathrm{n}_{\mathrm{zc}}\right)$ para Caso $3 / 152$

Figura 76. Parámetro de expansión $(\alpha)$ vs energía de pulso $(\mu \downarrow) / 152$

Figura 77. Imagen de sistema de posicionamiento y transductor fuerza desplazamiento / 160

Figura 78. Esquema de transductor fuerza-desplazamiento: placa fija, placa móvil y muestra / 160

Figura 79. Curva típica de carga vs desplazamiento en muestra de dióxido de silicio / 161

Figura 80. Mapas $\mu$-Raman, imagen de modificaciones estructurales / 163

Figura 81. Variación de volumen estimada utilizando $\mu$-Raman / 164

Figura 82. $\log (E / E o)$ vs $\log (\rho / \rho o)$, representado por puntos, y su ajuste lineal representado por la curva roja / 165

Figura 83. Imagen de modificaciones estructurales e improntas / 166

Figura 84. Funciones asociadas a la matriz constitutiva del material / 168

Figura 85.Subfunciones de Codigo1.m y fajuste1.m / 168

Figura 86. Funciones de mallador2.m, codigo2.m y fajuste2.m / 168

Figura 87. Funciones del modelo óptico / 168 



\section{Índice de Tablas}

Tabla i. Valores de constantes piezo-ópticas de niobato de litio.

Pág.

53

Tabla ii. Corrimiento en número de onda de los fonones del niobato de litio respecto de su posición característica en función de presión hidrostática......................................... 68

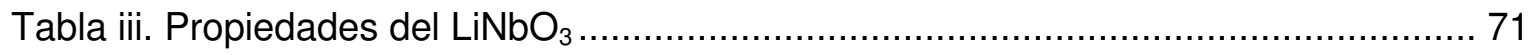

Tabla iv. Coeficientes de presión $d \omega / d p$ para $\mathrm{Nd}: \mathrm{Mg}: \mathrm{LiNbO}_{3}$ y $\mathrm{LiNbO}_{3} \ldots \ldots \ldots \ldots \ldots \ldots \ldots . . . \ldots 105$

Tabla v. Valores de d $\omega / \mathrm{dp}$ para dominio completo y dominio reducido de presiones.....109 Tabla vi.Valores obtenidos del ajuste: constantes de deformación potencial (e y f), errores cuadráticos medios de los distintos ajustes (ECM) y coeficiente numérico bajo presión

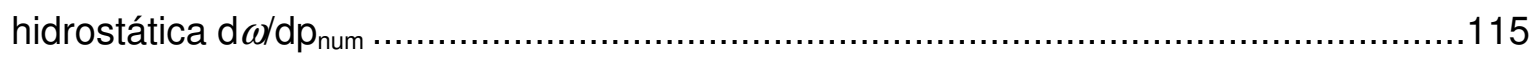
Tabla vii. Concordancia entre las desigualdades asociadas a los distintos valores de

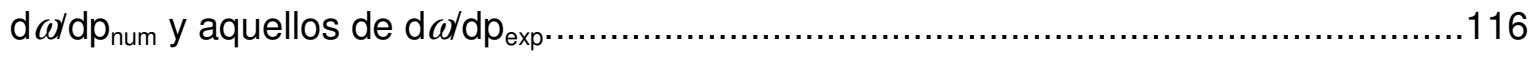

Tabla viii. Resultado del ajuste iterativo unidimensional. .........................................119

Tabla ix. Valores de $e, f$, f/e y $d \omega / d p$ obtenidas en el estudio bidimensional. ..................134

Tabla x. Energía de pulso (e) utilizada para las estructuras generadas ........................146

Tabla xi. Parámetros utilizando en el modelo numérico .........................................150 


\section{Lista de Símbolos y abreviaturas}

\section{Símbolo Término Unidad SI Definición}

\begin{tabular}{|c|c|c|c|}
\hline$a, b$ & $\begin{array}{l}\text { Radio horizontal y vertical de elipse en } \\
\text { modelo numérico }\end{array}$ & $\mu \mathrm{m}$ & \\
\hline$x_{c}, y_{c} y z_{c}$ & $\begin{array}{l}\text { Ejes de estructura cristalina de niobato de } \\
\text { litio }\end{array}$ & - & - \\
\hline \multicolumn{4}{|c|}{ 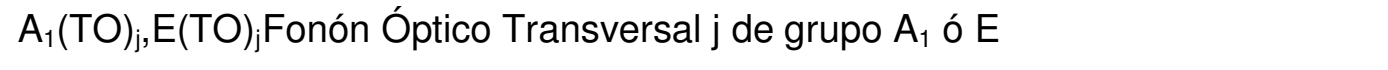 } \\
\hline \multicolumn{4}{|l|}{2} \\
\hline$\alpha, \beta$ & $\begin{array}{l}\text { Expansiones de la elipse en el modelo } \\
\text { numérico horizontal y vertical } \\
\text { respectivamente }\end{array}$ & 1 & $\Delta \mathrm{a} / \mathrm{a}, \Delta \mathrm{b} / \mathrm{b}$ \\
\hline$\beta_{p}$ & Constante de propagación & 1 & \\
\hline$d \omega / d p$ & $\begin{array}{l}\text { Variación de número de onda respecto a } \\
\text { presión hidrostática }\end{array}$ & $\mathrm{cm}^{-1} / \mathrm{Gpa}$ & \\
\hline $\begin{array}{l}d \omega / d p_{\text {num }} \\
d \omega / d p_{\text {exp }}\end{array}$ & $\begin{array}{l}\text { Variación de número de onda respecto a } \\
\text { presión hidrostática numérica y } \\
\text { experimental }\end{array}$ & $\mathrm{cm}^{-1} / \mathrm{Gpa}$ & \\
\hline$\varepsilon_{\mathrm{xc}}, \varepsilon_{\mathrm{yc}} \mathrm{y} \varepsilon_{\mathrm{zc}}$ & $\begin{array}{l}\text { Componentes normales del tensor de } \\
\text { deformación }\end{array}$ & 1 & $\Delta / / 1$ \\
\hline$\sigma_{\mathrm{xc}}, \sigma_{\mathrm{yc}}$ y $\sigma_{\mathrm{zc}}$ & $\begin{array}{l}\text { Componentes normales del tensor de } \\
\text { tensiones mecánicas }\end{array}$ & Gpa & \\
\hline$\Delta \omega\left(\mathrm{Z}_{\mathrm{c}}, \mathrm{x}_{\mathrm{c}}\right)$ & $\begin{array}{l}\text { Corrimiento en número de onda de un } \\
\text { fonón respecto de su posición nominal }\end{array}$ & $\mathrm{cm}^{-1}$ & \\
\hline$n, n_{1}, n_{0}$ & $\begin{array}{l}\text { Índice de refracción, del núcleo, del } \\
\text { sustrato de una guía de onda, } \\
\text { respectivamente }\end{array}$ & 1 & Pág. 18 \\
\hline$d$ & Ancho de guía de onda plana & - & Pág. 18 \\
\hline
\end{tabular}


Símbolo Término

$\frac{\Delta V}{V} \quad$ Variación de Volumen

$e_{j}, f_{j} \quad \begin{aligned} & \text { Constantes de Deformación Potencial del } \\ & \text { fonón } A_{1}(T O)_{j}\end{aligned}$

$e_{i}^{\prime}, f_{i}^{\prime}$

$\lambda \quad$ Longitud de onda

E,B Vectores de campo eléctrico y magnético

k Vector de Poynting

$\phi(x, y)$ Distribución de amplitud de campo 1

V Dirección de traslación de muestra

$\omega_{\mathrm{h}} \quad$ Cintura del haz

$F_{0}, F_{1}, F_{2} \quad$ Posición de Foco

$\mathrm{x}_{\mathrm{cf}} \quad$ Profundidad de enfoque

$c_{g} \quad$ Dirección de guía de onda

C Velocidad de la luz

$\chi^{(\mathrm{j})} \quad$ Suceptibilidad de orden j

$\boldsymbol{r} \quad$ Vector posición

$C_{e}, C_{i}$

$T_{e}, T_{i}$

$\gamma \quad$ Parámetro que cuenta del intercambio de

$\gamma \quad$ energía entre iones y electrones

$Q(z) \quad$ Flujo de calor

Calor específico de electrones y iones,
respectivamente

respectivamente

Temperatura de electrones y iones, respectivamente

\section{Unidad SI Definición}

$$
\frac{\Delta a}{a}+\frac{\Delta b}{b}
$$

$\mathrm{cm}^{-1} \quad \Delta \omega=\mathrm{e}\left(\varepsilon_{\mathrm{x}}+\varepsilon_{\mathrm{y}}\right)+\mathrm{f} \varepsilon_{\mathrm{z}}$

$\mathrm{cm}^{-1} / \mathrm{Gpa}$

Nm Sección 2.1

$-\quad$ ExB

1

Pág 22

$\mu \mathrm{m}$

Pág 24

Pág 24

Pág 25 
20 Caracterización de guías de onda ópticas generadas con láser de femtosegundos en Niobato de Litio

\section{Símbolo Término}

\section{Unidad SI Definición}

$S \quad$ Fuente de calor láser u Desplazamiento de puntos de un sólido

$u_{x}, u_{y}$ y $u_{z} \quad$ Desplazamientos en las direcciones $x, y$ y $z$

dl diferencial de distancia entre dos puntos de
sólido deformable

$\sigma_{i j} \quad$ Tensor de tensiones mecánicas

Pa Pág 30

$\varepsilon_{i j} \quad$ Tensor de deformaciones mecánicas

$1 \quad$ Pag 30

$\left[C_{i j}\right] \quad$ Matriz constitutiva

Pa Pág 31

$\left[S_{i j}\right] \quad$ Inversa de matriz constitutiva

$1 \quad$ Pág 31

$\lambda_{L} y \mu_{L} \quad$ Constantes de Lamé

G Módulo de sizalladura Ga

$X \quad$ Fuerzas de volumen N

$p_{i j k l} \quad$ Tensor de coeficientes piezo-ópticos

e Energía

ev Pág 34

h $\quad$ Constante de Planck

$v \quad$ Frecuencia de vibración de estructura $\mathrm{Hz}$ molecular

$\omega \quad$ Número de onda $\mathrm{cm}^{-1}$

$D_{1}, D_{2}, D_{3}, D_{4}$ Direcciones según estructura cristalina

$\mathrm{e}_{\mathrm{p}} \quad$ Energía de pulso $\quad \mu \mathrm{J} \quad$ Pág 44.

$\bar{P} \quad$ Potencia óptica promedio de tren de pulsos $\quad$ W

$n_{\mathrm{p}} \quad$ Número de pulsos $\quad 1$

$\sigma_{l m} \quad$ Tensiones mecánicas tangenciales en un
plano $I m$, normal a / y paralela a $m$ 
Símbolo Término

\begin{tabular}{|c|c|c|}
\hline$\varepsilon_{l m}$ & Deformaciones tangenciales en plano Im & Pág 58 \\
\hline$H_{i}(x, y)$ & $\begin{array}{l}\text { Funciónes de forma correspondiente al } \\
\text { nodo i. }\end{array}$ & \\
\hline$u_{x i} u_{y i}$ & $\begin{array}{l}\text { Desplazamientos nodales ( } \mathrm{x} \text { e } \mathrm{y} \text { ) para el } \\
\text { nodo } i\end{array}$ & \\
\hline$[B]$ & Matriz cinemática del modelo elástico & \\
\hline$\left[\mathrm{K}^{\mathrm{e}}\right],[\mathrm{K}]$ & $\begin{array}{l}\text { Matriz de rigidez elemental de modelo } \\
\text { elástico y matriz de rigidez global del } \\
\text { mismo, respectivante }\end{array}$ & \\
\hline$A_{e}$ & Área de elemento & \\
\hline$\{f\}$ & $\begin{array}{l}\text { Vector de fuerzas externas en modelo } \\
\text { elástico }\end{array}$ & \\
\hline$A$ y $B$ & $\begin{array}{l}\text { Tamaño de dominio horizontal y vertical } \\
\text { respectivamente }\end{array}$ & Pág 63 \\
\hline$\Delta z_{c i}, \Delta x_{c i}$ & $\begin{array}{l}\text { Desplazamiento nodales del cuarto de } \\
\text { elipse }\end{array}$ & Pág 64 \\
\hline$\varepsilon_{t o l}$ & $\begin{array}{l}\text { Diferencia de deformación entre el modelo } \\
\text { reducido y el modelo ideal. }\end{array}$ & Pág 65 \\
\hline$E_{k}$ & Módulo de elasticidad & \\
\hline$v^{p}$ & Coeficiente Poisson & \\
\hline
\end{tabular}

\section{Abreviatura Término}

\begin{tabular}{ll}
\hline$N A$ & Apertura Numérica \\
$E C M$ & Error Cuadrático Medio \\
$E T C$ & Error Total Cuadrático, Pág. 93 \\
$T P D L$ & Teoría Potencial de Deformación Lineal \\
$M E$ & Modificación Estructural
\end{tabular}





\section{Introducción}

\subsection{Estado del arte}

\subsubsection{Guías de onda fabricadas con láser de pulsos ultra- cortos}

$\mathrm{E}$ I desarrollo de los láseres de pulsos ultra-cortos controlados (de cientos de femtosegundos de duración) ha abierto nuevos horizontes de estudio en física, química e ingeniería de materiales. En particular, la interacción de éstos pulsos con materiales sólidos transparentes ha motivado, desde 1996 [DA96], la fabricación de guías de onda de una forma relativamente rápida, mediante un solo paso y en estructuras tridimensionales. El método que comúnmente se utiliza, consiste en desplazar el láser, focalizándolo mediante un objetivo microscópico, dentro del material. Generando así, una modificación estructural localizada que cambia el índice de refracción y permite "inscribir" dichas guías. Durante el mencionado proceso de fabricación, el desplazamiento del foco se debe realizar a una velocidad de algunas decenas de micrones por segundo, de modo de lograr, a medida que este se desplaza, la superposición de los volúmenes focales del láser para obtener una modificación apropiada.

Desde que se comenzó a desarrollar la técnica de escritura de guías de onda con láser de femtosegundos, tomó relevancia el estudio empírico de este proceso de fabricación y varios autores [MI06] han propuesto que ésta es la mejor estrategia para optimizarlo. Ya que debido a los diversos procesos no-lineales involucrados, es muy complejo desarrollar modelos adecuados para predecir el estado final del material por primeros principios [MI06,GA12,GA06,YAN08,RI04].

Aplicando este método de fabricación de guías de onda y utilizando como base materiales ópticos apropiados, es posible fabricar diversos circuitos "fotónicos" como por ejemplo: láseres, amplificadores ópticos y sensores, entre otros. Uno de los materiales más utilizados para este fin es el cristal de niobato de litio. El mismo, es crecido artificialmente bajo atmósfera controlada y posee una estructura cristalina de celda unidad trigonal. La misma se compone de un átomo de $\mathrm{Li}$, un átomo de $\mathrm{Nb}$ y tres de oxigeno $\left(\mathrm{LiNbO}_{3}\right)$. Debido a dicha estructura, este cristal tiene propiedades anisotrópicas. 
Esto implica que las diferentes propiedades físicas que lo caracterizan, dependen en general, de la orientación en que se estudian.

Por otro lado, es posible doparlo con tierras raras $(\mathrm{Nd}, \mathrm{Er}, \mathrm{Yb})$ para transformarlo en un material activo y luminiscente. Esto, permite utilizarlo como medio activo en láseres de estado sólido. También es dopado con metales de transición ( $\mathrm{Mg}, \mathrm{Ti}, \mathrm{Cr}$ ), para disminuir el daño por efecto foto-refractivo cuando se requiere propagar en su seno altas intensidades de luz[LEN07].

Además, el niobato de litio se clasifica entre los materiales "electro-ópticos". Esta propiedad permite fabricar en su seno dispositivos fotónicos de "control activo", como por ejemplo, moduladores de luz (MIOC), componentes que resultan de gran utilidad en sensores fotónicos y comunicaciones ópticas [OKA05].

Por todas estas razones, este es uno de los materiales más utilizado para la fabricación de guías de onda.

Hasta la actualidad, se han generado dos tipos de guías de onda utilizando escritura láser con pulsos de femtosegundos de baja tasa de repetición (1KHz) [WILL05]. Por un lado, las que presentan una zona de aumento de índice de refracción en la región donde se enfocó el láser (Guías Tipo I). Y por otro lado, las que presentan, en la región donde se enfocó el láser, una modificación estructural y disminución muy pronunciada del índice de refracción, mientras que en la región lindante a la misma, presentan un aumento del índice de refracción (Guías Tipo II).

Lo que diferencia la generación de cada tipo de guía, para un mismo material y tiempo de pulso (cientos de femtosegundos), es principalmente la energía de pulso que se utilice. Las guías tipo I se fabrican con energía de pulso relativamente baja (en general menores a $0.5 \mu \mathrm{J}$ ) y las guías tipo II con energía de pulso relativamente altas (mayores a $0.5 \mu \mathrm{J}$ ).

Algunos aspectos relevantes de las guías tipo I son los siguientes [GA06]:

- no todos los materiales transparentes admiten su fabricación;

- en general, no soportan temperaturas mayores a $400{ }^{\circ} \mathrm{C}$, lo que implica que si son sometidas a temperaturas de este orden, se desvanecen;

- tienen la ventaja de que se han reportados bajos valores de perdidas ópticas en ellas (aproximadamente $0.01 \mathrm{db} / \mathrm{cm}$ ).

En cuanto a las guías tipo II, se pueden mencionar las siguientes características [SCHA01]:

- en general, se pueden fabricar en cualquier material transparente, observándose, para los distintos materiales, similares características morfológicas de la zona del material que interactúa directamente con el láser. Esta zona presenta un bajo índice de refracción, mientras que en la zona adyacente se registra, en general, un incremento del mismo;

- no se desvanecen a altas temperaturas $(>500 \stackrel{\circ}{\circ} \mathrm{C})$. 
- tienen la desventaja que se midieron en ellas valores de perdidas mayores a las tipo I $(0.2 \mathrm{a} 1 \mathrm{db} / \mathrm{cm})$.

En las guías Tipo II, se han realizado estudios tanto de la modificación estructural como de la zona de guiado, utilizando los más diversos métodos de análisis. De la zona estructuralmente modificada, es de interés, en general, la nueva estructura formada bajo condiciones de temperatura y presión no convencionales. En cambio, de la zona de guiado de luz adyacente a dicha modificación, es de interés el campo de índice de refracción, como así también las pérdidas ópticas de guiado. Aunque estas dos últimas propiedades se encuentran vinculadas tienen distinto origen y consecuencias en el diseño de guías de onda[TO14].

Los métodos que se han utilizado para estudiar la región de modificación estructural son los siguientes.

AFM. Este método se utilizó para mapear la zona de modificación estructural generada en dióxido de silicio [VU08]. El mismo permitió detectar, dentro de esta región, pequeñas burbujas ("voids") de algunos micrones de diámetro que se ubican centradas respecto a las modificaciones. Esta modificación se observa con microscopía óptica y posee forma elíptica alargada de aproximadamente 30/50 $\mu \mathrm{m}$ de extensión.

A su vez, se midió la geometría de la zona modificada, con alta resolución espacial (20 $\mathrm{nm}$ ) en estructuras fabricadas en $\mathrm{SiO}_{2}$. Para ello, se utilizó una combinación de procesos de deposición química y AFM [TAY03].

Microscopía de dispersión de electrones (SEM). Utilizando esta técnica se relevaron cambios en la topografía de la zona modificada estructuralmente por pulsos láser de femtosegundos en $\mathrm{SiO}_{2}[\mathrm{POU} 03]$.

$\boldsymbol{\mu}$-Luminiscencia y $\boldsymbol{\mu}$-Raman. Estas técnicas se utilizaron para determinar la estructuración y densidad en la zona modificada por el láser de femtosegundos en diferentes materiales: $\mathrm{SiO}_{2}, \mathrm{GeO}_{2}$ y $\mathrm{LiNbO}_{3}$ [BRESS11, vU08, BEN09].

Los métodos utilizados para al estudio de la región de guiado de luz en las estructuras en cuestión, son los empleados en general para estimar la variación de índice de refracción, en cualquier guía de onda (aunque la aplicabilidad de un determinado método depende, en general, del índice de refracción del material). Estos métodos y sus principales fundamentos, se mencionan a continuación (para evitar una extensión innecesaria, no se mencionan todas las variantes de los mismos).

Microscopía interferométrica de corrimiento de fase. Las regiones de guiado de luz, lindantes a la modificación estructural, fueron estudiadas utilizando esta técnica en $\mathrm{SiO}_{2}$ [POU03]. Esto reveló la clara existencia de campos de deformaciones en dichas regiones. A su vez, se observaron distintas características de dichos campos para distintas 
energías de pulso. En guías de femtosegundos fabricadas en $\mathrm{LiNbO}_{3}$ también se midió el campo de índice de refracción utilizando esta técnica [BUR07].

Interferometría y holografía digital. Utilizando un interferómetro y holografía digital, se ha medido el perfil de índice de refracción en guías de onda fabricadas con láser de femtosegundos en vidrios convencionales [FERR05]. En este novedoso método pueden destacarse las siguientes ventajas: una alta resolución espacial, reducidos tiempos de adquisición, permite una medición de varias componentes de índice de refracción con una buena resolución, y no requiere desplazamientos sistematizados.

Medición de Campo Cercano Refractado (RNF, Refracted Near Field Measurements). Esta técnica fue desarrollada, en sus comienzos, para medir el campo de índice de refracción de fibra óptica y requiere una disposición relativamente compleja de la muestra. La misma, se utilizó para medir el campo de índice de refracción principalmente en guías fabricadas en $\mathrm{SiO}_{2}(n \sim 1.4)$ [OBER98]. Para materiales de mayor índice de refracción, como por ejemplo niobato de litio (n 2.2) no se reportaron medidas. Se estima que esto se debe a que el método se basa en la medición del ángulo de refracción y cuando aumenta el índice de refracción del material se dificulta la medida del mismo.

$\mu$-Luminiscencia y $\mu$-Raman. Estas técnicas se han utilizado para explorar, mediante mapeos, la zona de guiado generada con láser de femtosegundos [RO09_2, BRESS11, vU08], pero no se ha establecido una relación cuantitativa que permita estimar el campo de índice de refracción a partir de las mismas.

$\mu$-Reflectividad. Este método consiste en medir el cambio de la reflexión de Fresnel (en una dirección perpendicular a la superficie) en el extremo de la guía de onda. Para ello, es necesario medir la intensidad incidente y la reflejada en esta superficie para obtener la variación del índice de refracción. Tiene una resolución en el índice de refracción de $10^{-5}$ y es relativamente sencillo de aplicar. Existen trabajos donde se aplica a guías de onda fabricadas con láser de femtosegundos en vidrios [BHAR05].

Difracción de Rayos X. En zonas de guiado tipo II generadas en YAG se detectaron cambios en la difracción de Rayos $\mathrm{X}$, lo que es consistente con la existencia de una deformación de la estructura cristalina [HENO9].

Métodos de cálculo numérico inverso de Helmholtz. Este método consiste en resolver por métodos numéricos, la ecuación de Helmholtz en forma inversa. Se puede aplicar a guías monomodo y se ha utilizado para estimar el campo de índice de refracción en guías de onda fabricadas con láser de femtosegundos en $\mathrm{SiO}_{2}$ [SZA06]. 


\subsubsection{Origen de guías de onda tipo II}

En la literatura existente, se ha propuesto que las guías de onda tipo II tienen su origen, principalmente, en una deformación mecánica del cristal, producida por una expansión originada como consecuencia de la interacción láser pulsado-material [BUGRE07, NOBUR04]. Es decir que, se asume que dicha deformación residual genera, por fotoelasticidad, un aumento localizado de índice de refracción en las zonas lindantes, lo que permite el guiado de luz. Esta hipótesis se ha sustentado fundamentalmente en tres factores:

- La luz propagada en guías de onda tipo II está mayormente polarizada en dirección vertical [WILL05].

- Utilizando un modelo de elementos finitos, en donde, una elipse se expande en forma estática en un medio elástico, se concluyó que la deformación residual genera, por foto-elasticidad, un aumento de índice de refracción en regiones lindantes a la elipse, donde se han medido luz guiada[NOBUR04].

- Se han detectado corrimientos en los fonones Raman en la zona de guiado. Este fenómeno también es consistente con una deformación de la red cristalina [RO09].

A su vez, los estudios realizados con microscopía interferométrica de corrimiento de fase y difracción de Rayos $\mathrm{X}$ citados en los párrafos anteriores, también son consistentes con una deformación de la red cristalina en la zona de guiado.

Por otro lado, se han fabricado otro tipo de guías de onda utilizando una alta tasa de repetición de pulsos de femtosegundos $(25 \mathrm{MHz})$. Las mismas tienen una conformación cualitativamente diferente a las de baja tasa de repetición $(1 \mathrm{KHz})$. Esto se debe a que, para una alta tasa de repetición ocurren fenómenos térmicos y cambios estructurales más extendidos. Como consecuencia, esta interacción forma varias zonas de modificación estructural en la dirección de propagación del láser y el guiado de luz se presenta en su parte superior. En estas modificaciones, se ha reportado guiado de luz de polarización horizontal y se realizaron mapas bidimensionales Raman [RO08], observando, estos últimos, corrimientos espectrales en las regiones de guiado.

\subsubsection{Espectroscopia Raman bajo deformaciones}

En la actualidad, existe escasa información acerca de cómo se comporta el espectro Raman del niobato de litio en presencia de deformaciones. Se publicaron trabajos en la década de 1980 [JAY86, Fl84] donde se analizó la espectroscopía Raman en niobato de litio puro bajo presión hidrostática hasta altas presiones ( 0 a $20 \mathrm{Gpa}$ ) de a intervalos de $0.1 \mathrm{Gpa}$ aproximadamente.

Aunque al comenzar el presente trabajo de investigación no se había reportado el comportamiento Raman del niobato de litio bajo deformaciones generales (nohidrostáticas), sí existía una importante cantidad de estudios experimentales y trabajos 
teóricos, realizados en el marco de la Teoría Potencial de Deformación ${ }^{* 1}$, en otros materiales cristalinos como silicio, sulfato de cadmio y cuarzo [TE71, TE73, BRI76, WO96, A81]. Algunos de estos materiales como el cuarzo tienen una estructura cristalina similar al niobato de litio. Esto permitió analizar de forma análoga, y dentro de un marco teórico apropiado, el comportamiento piezo-espectroscópico de este material (cómo se desplazan los fonones ópticos Raman en función del campo de deformaciones). Por consiguiente, desde un punto de vista experimental, se puede decir que el niobato de litio no se encontraba caracterizado piezo-espectroscópicamente para deformaciones generales (solo se encontraba caracterizado experimentalmente bajo presión hidrostática) al comenzar este trabajo de investigación. Cabe mencionar, que al momento de la redacción de esta tesis se ha publicado un trabajo en el que se estiman las constantes de deformación potencial del niobato de litio puro[PE13].

\subsubsection{Modelos numéricos aplicados al estudio de guías de onda fabricadas con láser de femtosegundos}

Sólo en escasos trabajos [BUR07, BURHA07, WILL05] se ha utilizado un modelo elástico numérico para el estudio cualitativo de guías de onda tipo II en niobato de litio. En este modelo se aplica una expansión mecánica estática producida por un cambio de volumen en la zona de interacción directa. El mismo se ha utilizado principalmente para estudiar el origen de esta clase de guías de onda. Concluyendo que el proceso más importante que interviene en su conformación es una expansión mecánica. Inicialmente propusieron una expansión proporcional (la elipse final es proporcional a la inicial) de acuerdo al cambio de volumen previamente estimado: $\Delta \mathrm{V} / \mathrm{V}=5 \times 10^{-3}$ [BUR07]. Mientras que en el siguiente trabajo [BURHA07], propusieron expandir térmicamente una elipse central (utilizando una temperatura artificial) hasta que la misma alcance el cambio de volumen mencionado. En este último modelo, la elipse se expande bajo la "presión" del medio, y como este último no es isotrópico, la expansión es diferente a la proporcional. Pero, teniendo el medio (cristal de niobato de litio), una diferencia de un $10 \%$ entre la rigidez en la dirección horizontal y la vertical, se puede inferir con un criterio conservador, que la expansión resultante en este caso, no será radicalmente distinta a la expansión proporcional.

\subsubsection{Indentación instrumentada en estructuras fabricadas con láser de femtosegundos.}

La técnica de indentación instrumentada se deriva de la conocida técnica macroscópica de penetración ó indentación utilizada para determinar dureza en materiales. Como consecuencia, el marco teórico básico para analizar el fenómeno es la mecánica de contacto, que fue iniciada por Hertz en la década de 1880 [FI06].

\footnotetext{
${ }^{1}$ Marco teórico general para la dependencia entre la espectroscopía Raman y el campo de deformaciones.
} 
Esta técnica, se basa en penetrar el material con una punta micrométrica de diamante calibrada, midiendo simultáneamente, el desplazamiento y la carga aplicada con una alta resolución $(0.1 \mathrm{~nm}$ y $10 \mu \mathrm{N}$ respectivamente). La punta puede tener diversas geometrías (esférica, piramidal cuadrada, piramidal triangular, etc.). Analizando la curva "carga vs. desplazamiento" generada, se pueden extraer numerosas propiedades mecánicas incluyendo: módulo elástico $(\mathrm{E})$, dureza $(\mathrm{H})$, rigidez a la fractura $(\mathrm{S})$ y comportamiento plástico del material a escala micrométrica, entre otras.

Los cristales, son buenos candidatos para ser estudiados con esta técnica. De hecho, para calibrar los ensayos de nanoindentación se utilizan, en general, muestras de dióxido de silicio.

Actualmente, existen trabajos donde se estudian las propiedades mecánicas del niobato de litio utilizando esta técnica [BHAR05, BA08]. En los mismos, se estima el módulo elástico, la dureza, la rigidez a la fractura y el comportamiento de dislocamiento bajo altas cargas de contacto.

En niobato de litio, no se han reportado a la fecha medidas del módulo elástico en la zona modificada con láser de femtosegundos. En cambio, la misma si se ha estudiado en dióxido de silicio [BELL06] y en otros vidrios [KON10]. En estos casos, se pudo medir con esta técnica, una variación del módulo de elasticidad del material en la zona modificada.

Por otro lado, se han desarrollado distintos métodos para estudiar deformaciones residuales a partir de esta técnica [YA08]. Éstos se pueden clasificar en dos clases: Los que utilizan la imagen de la impronta ó cráter de la indentación/penetración para inferir las deformaciones pre-existentes en el material y los que se basan en los cambios que experimenta la curva de carga-desplazamiento en presencia de deformaciones: la compresión genera un leve aumento de la rigidez del material y la tracción una disminución de la misma. Sin embargo, estas metodologías no se encuentran utilizadas para caracterizar deformaciones producidas con láser de femtosegundos.

\subsubsection{Resumiendo...}

Al comenzar el presente trabajo, los aspectos conocidos de guías de onda tipo II fabricadas con láser de femtosegundos, se pueden resumir en los siguientes ítems:

*Las guías de onda tipo II se generan producto de una expansión mecánica generada por la interacción entre el láser pulsado y el cristal. El orden de magnitud de esta expansión es conocido.

*Esta expansión genera un incremento de índice de refracción en sus adyacencias. 
*Para el estudio cualitativo de este tipo de guías, es posible utilizar un modelo elástico y estático, en donde se expande una elipse.

*En este tipo de estructuras de guiado, se han podido guiar luz polarizada con distinta orientación.

*Se detectaron corrimientos espectrales de los fonones $\mu$-Raman en las guías mencionadas y los mismos poseen una correlación, en ausencia de cambios estructurales, con el estado de deformación de la estructura cristalina.

*Se ha reportado un comportamiento lineal del corrimiento espectral (habitualmente expresado en número de onda) de los fonones del niobato de litio puro bajo presión hidrostática, hasta $10 \mathrm{GPa}$.

*La densidad, el índice de refracción y el módulo de elasticidad disminuyen en la región estructuralmente modificada por la interacción con el láser de femtosegundos.

\subsection{Interrogantes y problemas a resolver}

Al iniciar este trabajo de tesis existían los siguientes interrogantes y problemas a resolver:

i_El espectro Raman del niobato de litio no se encontraba caracterizado piezo espectroscópicamente para deformaciones generalizadas. Es decir, que no se conocía como se desplazan los fonones Raman $\mathrm{A}_{1}(\mathrm{TO})$ en función de una deformación generalizada: en qué proporción afecta cada componente normal de deformación $\left(\varepsilon_{x}, \varepsilon_{y} y\right.$ $\left.\varepsilon_{z}\right)$ a los corrimientos de estos fonones.

ii_ No se habían reportado trabajos donde se relacionen las deformaciones residuales con los corrimientos Raman medidos en guías de onda fabricadas con láser de femtosegundos, en particular, en niobato de litio. Por consiguiente, tampoco se había analizado si es posible utilizar dicho modelo numérico para conocer/ajustar de forma cuantitativa el campo de deformaciones que conforma una guía de onda fabricada por el láser de femtosegundos.

iii_ No se encontraban reportadas, explícitamente, las características geométricas de la expansión mecánica producida por la interacción láser de femtosegundos-material.

iv_ No se encontraba estudiado el comportamiento del espectro Raman del cristal $\mathrm{Nd}: \mathrm{Mg}: \mathrm{LiNbO}_{3}$ bajo presión hidrostática.

$V_{-}$No se habían reportado mapas bidimensionales de los corrimientos Raman en guías de femtosegundos de baja tasa repetición $(1 \mathrm{KHz})$. 


\subsection{Objetivos y motivación}

Los objetivos de este trabajo de investigación han sido:

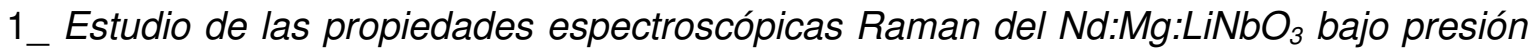
hidrostática y comparación con el $\mathrm{LiNbO}_{3}$ puro.

El Nd:Mg:LiNbO ${ }_{3}$ es un cristal muy utilizado en fotónica integrada [QUI09], principalmente para conformar estructuras guiadas activas como láseres y amplificadores en su seno. Por consiguiente es importante conocer el comportamiento de su espectro Raman bajo presión hidrostática, ya que los coeficientes de presión hidrostática Raman pueden ser utilizados para determinar campos de deformaciones en este cristal. Por estas razones, se propuso estudiar la variación del espectro Raman del $\mathrm{Nd}: \mathrm{Mg}: \mathrm{LiNbO}_{3}$ en función de la presión hidrostática (hasta $6 \mathrm{Gpa}$ ).

2_Caracterizacion de las deformaciones y variaciones en el índice de refracción de guías de onda fabricadas con láser de femtosegundos.

La fabricación de guías de onda con láser de femtosegundos es una buena alternativa a los métodos establecidos (ej. Difusión de Ti, Implantación lónica, etc. [BUL84, JA85]), por ser un método relativamente rápido, de un solo paso y con capacidad de inscribir guías ópticas tridimensionales en el seno de un material transparente [OSE06, MI06] (aunque este último aspecto aún no está totalmente resuelto por la variación del volumen focal con la profundidad de enfoque, entre otros factores [MO11]). Por otro lado es conocido que el campo residual de deformaciones define el campo de índice de refracción de las guías de onda tipo II y que, a su vez, este último representa la principal característica a tener en cuenta en el diseño de circuitos fotónicos (acopladores, filtros, amplificadores, etc.) [OKA05]. Por estos motivos, se propuso estudiar mediante métodos experimentales y computacionales, el campo de deformaciones y su campo de índice de refracción asociado, en estas guías de onda. Para tal fin, se vincularon: un modelado numérico elástico, mapeos espectroscópicos micro-Raman y mapeos de distribución de intensidad de la luz en estas estructuras. Para esto, se utilizaron guías fabricadas en $\mathrm{Nd}: \mathrm{Mg}: \mathrm{LiNbO}_{3}$.

Aunque el presente trabajo se enfoca en estudiar el campo de deformaciones en niobato de litio, la técnica se puede extrapolar a otros materiales transparentes para determinar sus propiedades piezo-espectroscópicas. Las mismas tienen aplicación en diversas áreas como ser micro-electrónica y micro-mecánica cuando se trabaja con cristales como el Cuarzo $\left(\mathrm{SiO}_{2}\right)$ y el Silicio ( $\left.\mathrm{Si}\right)$. Esto se debe a que es posible obtener información, en teoría, absoluta del estado de deformaciones de una estructura cristalina utilizando espectroscopia Raman[A81].

Complementariamente, se propuso determinar las constantes de deformación potencial Raman para el $\mathrm{Nd}: \mathrm{Mg}: \mathrm{LiNbO}_{3}$. 
32 Caracterización de guías de onda ópticas generadas con láser de femtosegundos en Niobato de Litio

3_Desarrollo de un método directo (sin iteraciones) para medir los campos de deformaciones utilizando mapeos $\mu$-Raman, aplicable en guías tipo II, para determinar el campo de índice de refracción.

Una vez estimadas las constantes de deformación potencial, a partir de los mapeos $\mu$-Raman, se propuso aplicar un método directo (sin iteraciones de cálculo) para estimar los campos de deformaciones e índice de refracción en guías de onda tipo II.

4_ Estudio de la polarización de la luz guiada en guías tipo II utilizando diferentes energías de pulso y correlación de resultados experimentales con resultados numéricos para distintas expansiones mecánicas.

Debido a que se ha reportado en distintos trabajos el guiado de polarización horizontal y vertical, se propuso realizar un estudio de polarizaciones guiadas para guías fabricadas con diferentes energías de pulso láser. A su vez, se aplicaron distintas expansiones en el modelo numérico para luego correlacionarlas con los resultados experimentales, considerando distintos valores de energía de pulso.

\subsection{Enfoque y metodología.}

El estudio realizado se puede clasificar como explorativo, ya que existe poca bibliografía enfocada en este tema. A su vez, debido a la alta complejidad de la interacción ultrarápida [GA12], este estudio es de carácter fenomenológico y se orientó principalmente a conocer las características de la expansión que ocurre luego de dicha interacción. Conceptualmente, esto se realizó comparando los resultados de las mediciones experimentales con las leyes de variación predichas por los modelos numéricos.

En el siguiente diagrama, se presenta un esquema de las técnicas experimentales y numéricas que se utilizan en este trabajo de tesis. 


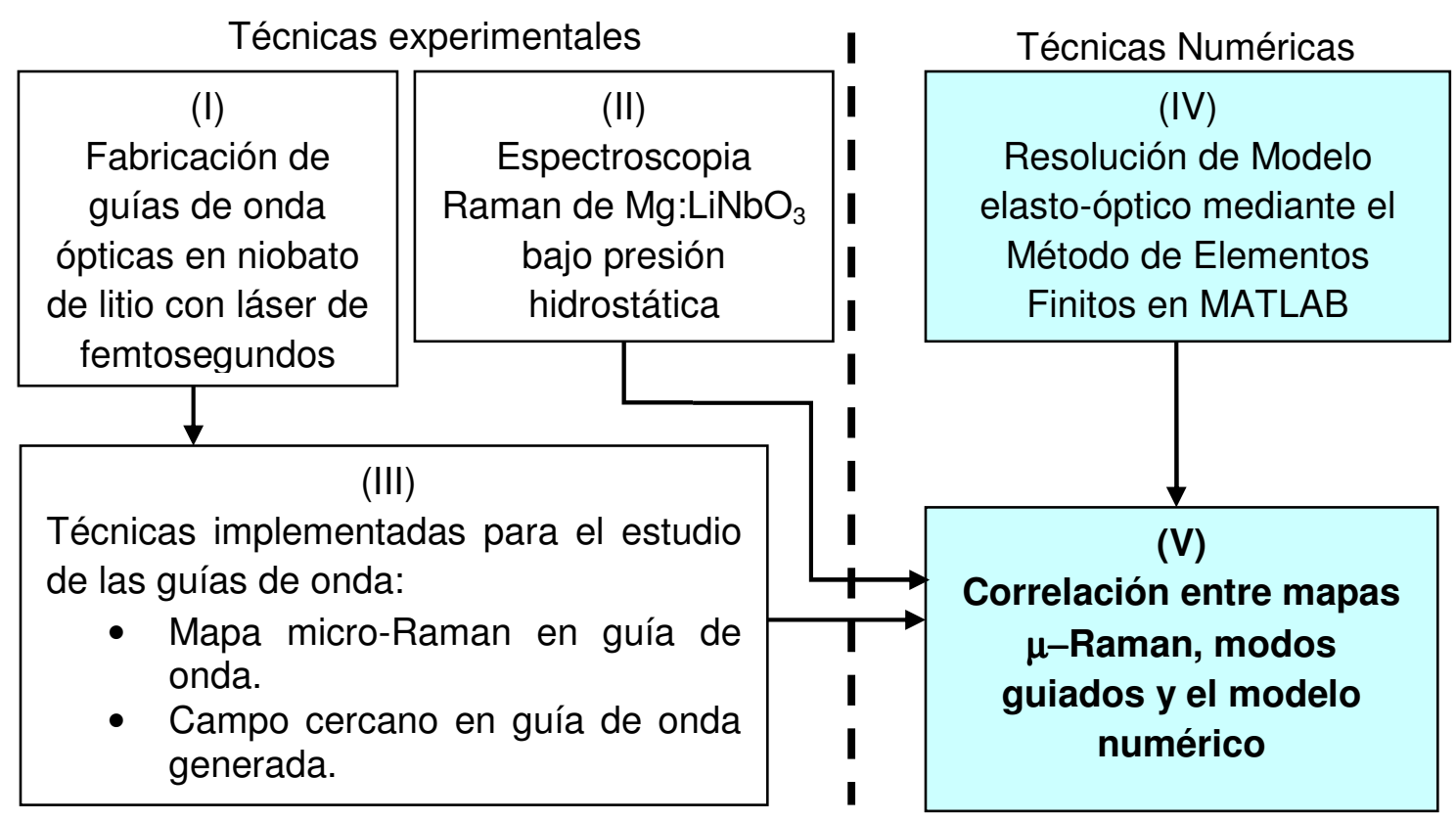

Esquema de técnicas empleadas

\section{Técnicas experimentales}

(I) Las guías de onda estudiadas se fabricaron con un láser de femtosegudos y una estación de posicionamiento micrométrica.

(II) Las medidas de los corrimientos de los fonones Raman bajo presión hidrostática se utilizaron en un primer análisis para calcular el orden de magnitud y característica de la expansión.

(III) Para la caracterización de las estructuras fabricadas se utilizaron técnicas de mapeo $\mu$-Raman y medidas del campo cercano de la guía mediante el método de acople "Endfire".

\section{Técnicas numéricas}

(IV) Se generó un modelo numérico en el cual se aplica una expansión arbitraria obteniéndose como resultado: el campo de deformaciones, el campo de índice de refracción y los modos guiados.

(V) Se ajustaron o correlacionaron los resultados de las técnicas experimentales con los del modelo numérico. Esto permite obtener, bajo determinadas suposiciones: información del campo residual de índice de las guías de onda, las características geométricas de la expansión y la variación del módulo elástico en función de la densidad local. 


\subsection{Estructura y organización de la Tesis}

En el Capítulo 2, se describen los fundamentos físicos y el marco teórico:

- La interacción entre láseres de pulsos ultra-cortos (cientos de femtosegundos) y materiales transparentes en general y algunas particularidades de la interacción con el niobato de litio.

- La definición de deformaciones mecánicas, tensiones y el efecto piezo-óptico

En el capítulo 3 se describen: las técnicas experimentales, los fundamentos de las mismas, los equipos empleados y los materiales utilizados. En particular:

- Se detallan los procedimientos implementados para fabricar guías de onda tipo II en niobato de litio utilizando un láser de pulsos ultra-cortos.

- Se presentan las características de las muestras utilizadas y se describe el procedimiento de pulido de muestras

- Se mencionan los fundamentos de la espectroscopia Raman y su relación con el estado de deformación de la estructura cristalina en el tipo de material estudiado. Se presenta la Teoría de Deformación Potencial (que relaciona la posición de los fonones Raman con el campo de deformaciones).

- Se describe el procedimiento utilizado para realizar mapeos $\mu$-Raman.

- Se describen los sistemas utilizados por el Dr. A. Goñi (UAB) para realizar ensayos de presión hidrostática.

En el capítulo 4 se describen los modelos numéricos que se utilizaron para resolver el problema elástico y de modos guiados y las aproximaciones implementadas (matriz de elasticidad ortotrópica, deformaciones planas, etc.). También se describen los procedimientos asociados a la programación de ambos modelos numéricos mediante el método de elementos finitos y verificaciones de los códigos generados. Entre otros procedimientos, se describe la elección del tamaño de dominio, la elección de tipo de elemento y los procedimientos iterativos. Los algoritmos se programaron en entorno MATLAB y se utilizaron los programas comerciales $A B A Q U S \circledast$ y R-SOFT® para verificar sus resultados.

En el capítulo 5 se presentan los resultados obtenidos y se divide en cuatro subsecciones:

5.1: Estudio $\mu$-Raman bajo presión hidrostática de $\mathrm{Nd}: \mathrm{Mg}: \mathrm{LiNbO}_{3}$. Se presenta el comportamiento de los fonones Raman del niobato de litio dopado con magnesio bajo presión hidrostática.

5.2: Análisis $\mu$-Raman de guías de onda fabricadas con láser de femtosegundos. Se presentan los resultados obtenidos mediante la comparación de los mapas de corrimiento Raman (unidimensional y bidimensional) con los modelos numéricos en diferentes guías de onda y con diferentes valores de parámetros del modelo. Se evalúan las técnicas 
numéricas y experimentales utilizadas para explorar los campos de deformaciones de las guías de onda estudiadas. Primeramente, se realiza un ajuste del mapeo unidimensional de los corrimientos Raman. En el mismo, los resultados numéricos se ajustan a los experimentales utilizando el método de mínimos cuadrados. Luego, se comparan los resultados numéricos con un mapa de corrimiento Raman bidimensional medido en guías de onda. A continuación, se realiza un ajuste entre el mapa de corrimiento Raman bidimensional y el modelo numérico.

5.3: Se presenta y aplica un método directo para calcular el campo de deformaciones y de índice de refracción, a partir de los mapas $\mu$-Raman.

5.4: Análisis de polarizaciones guiadas en guías fabricadas con láser de femtosegundos.

Se presenta un análisis cualitativo y comparativo entre: intensidades guiadas, reportadas en trabajos previos y el campo de índice de refracción obtenido para diferentes expansiones mecánicas en el modelo numérico. Luego, se presenta un análisis de las polarizaciones guiadas, utilizando el modelo numérico desarrollado, en guías de onda fabricadas con láser de femtosegundos para diferentes energías de pulso.

En el capítulo 6 se presentan las conclusiones de este Trabajo de Tesis, se resumen los resultados más relevantes y se mencionan trabajos programados para el futuro.

En el anexo A se presenta una aplicación de los mapeos $\mu$-Raman e indentación instrumentada para determinar la dependencia entre la densidad y el módulo de elasticidad.

En el anexo B se adjuntan los códigos utilizados en entorno MATLAB, en su mayoría de elaboración propia, para calcular los desplazamientos elásticos y modos guiados aplicando el método de elementos finitos. 



\section{Marco Teórico: Guías de onda, deformaciones y espectroscopia Raman}

En este capítulo se presentan los fundamentos de las guías de onda ópticas y generalidades del cálculo de modos guiados. También se mencionan algunos fenómenos físicos considerados relevantes de la interacción de láseres ultra-rápidos con materiales transparentes. A continuación, se definen las deformaciones y tensiones mecánicas en el marco de la Teoría Elástica. Como así también la relación entre el campo de deformaciones y el campo de variación de índice de refracción (efecto piezo-óptico).

\subsection{Guías de onda ópticas}

Teniendo en cuenta el modelo de rayos, las guías de onda ópticas (de aquí en adelante guías de onda) se pueden definir como estructuras que permiten el confinamiento de luz dentro de sus límites por Reflexión Total Interna (RTI). Este fenómeno ocurre cuando un rayo de luz, propagándose en un medio de índice de refracción $\mathrm{n}_{\mathrm{A}}$, incide en la interface entre este y un medio de índice de refracción $n_{B}$. En caso de que $n_{A}$ sea mayor que $n_{B} y$ que el ángulo de incidencia sea mayor al ángulo crítico, se produce una reflexión total de la luz denominada Reflexión Total Interna. Por consiguiente, se requiere un medio de un índice de refracción mayor, rodeado de uno de índice de refracción menor para confinar y guiar luz [LIN03]. El caso más simple de guía de onda lo representa una guía de onda "plana" simétrica. Su plano normal a la propagación de luz se esquematiza en la Figura 1. Tiene una zona con índice de refracción $n=n_{1}$ y dos zonas lindantes donde $n=n_{0}$, siendo $n_{1}>n_{0}$. En caso de que exista una diferencia adecuada entre $n_{1}$ y $n_{0}(\Delta n)$ y una distancia d adecuada, se puede propagar luz en la dirección z confinada en la zona central (donde $\left.n=n_{1}\right)$. En tal caso se dice que está luz es guiada. 


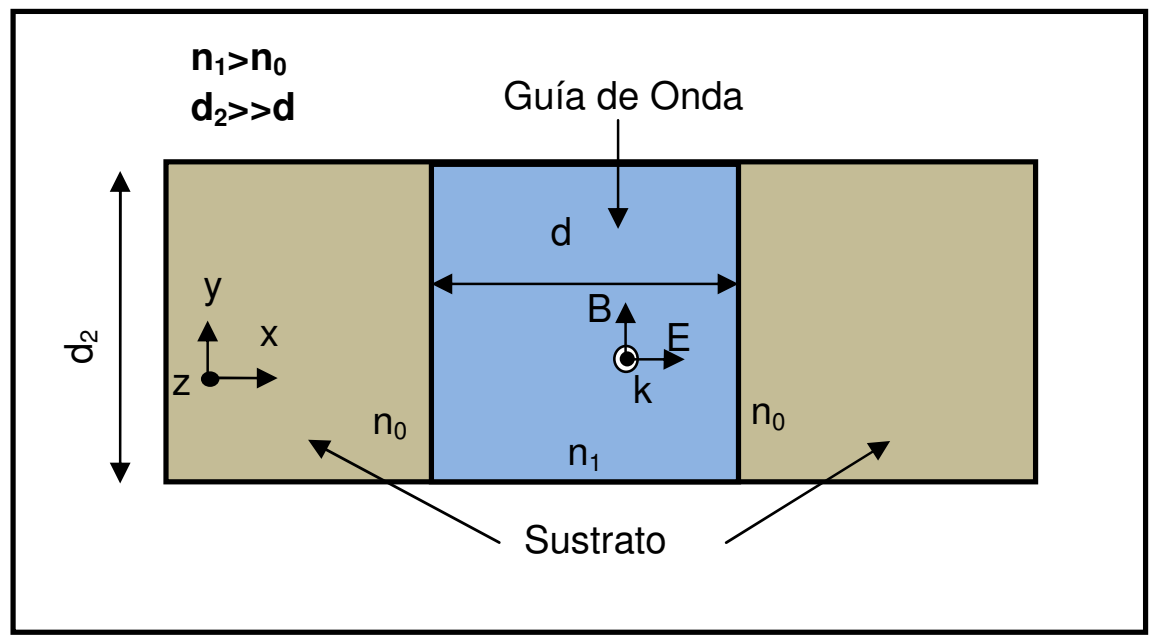

Figura 1. Esquema de guía de onda plana (corte tranversal). La luz se propaga en la dirección $z$, y los campos eléctrico y magnético están orientados en la dirección x e y, respectivamente.

De forma análoga, la luz también puede ser guiada en estructuras tridimensionales, en el seno de un material, a través de zonas donde exista un incremento de índice de refracción (Figura 2). Existiendo restricciones físicas para la magnitud de incremento de índice de refracción y las dimensiones de las guías. Para tener estructuras de guiado apropiadas, como generalidad, se puede mencionar que uno de los lados del área normal a la propagación de la luz debe tener dimensiones del orden de la longitud de onda propagada. Aunque la magnitud del incremento de índice de refracción también juega un papel importante en estas estructuras, como se mencionará más adelante.

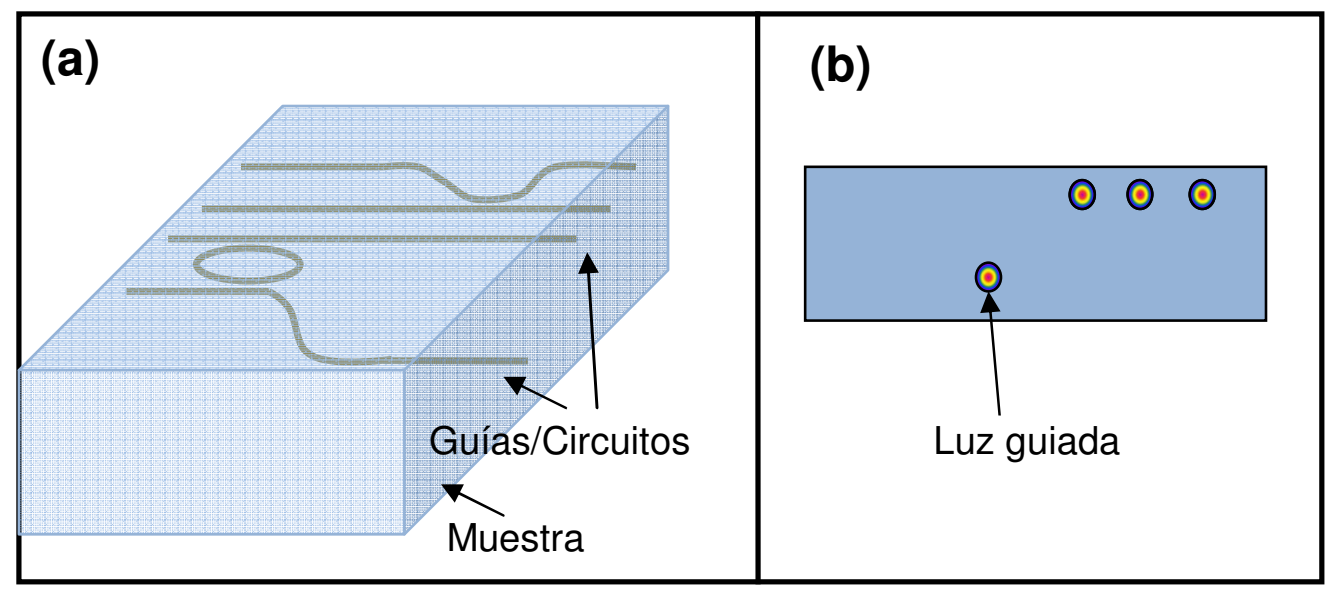

Figura 2. (a) Esquema de guías de onda tridimensionales. (b) corte transversal de la muestra, donde se esquematiza la distribución espacial de luz a la salida de la guía.

Las guías de onda se pueden fabricar con diversas tecnologías: algunas son multi-etapa, por ejemplo: difusión de metales y bombardeo de iones [BUL84,JA85] y otras son de una sola etapa como la escritura láser [OSE12]. Las guías de onda que se estudian en este trabajo fueron fabricadas mediante escritura con láser de femtosegundos. 


\subsubsection{Modos guiados.}

Analizando con mayor rigurosidad el campo electromagnético que se propaga en una guía de onda plana, este puede propagarse de forma Transversal Eléctrica (TE) ó Transversal Magnética (TM). En la Figura 3, se presenta un esquema de cada uno de estos campos propagados.

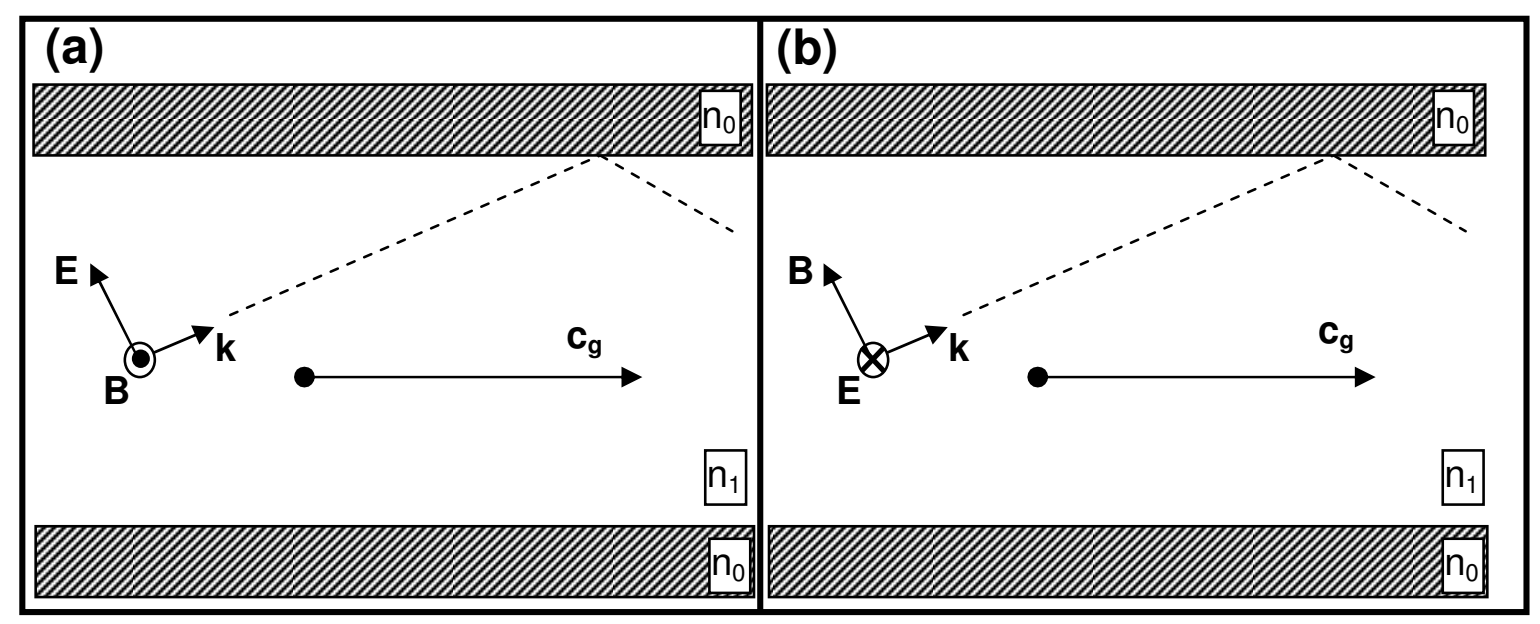

Figura 3. (a) Esquema de ondas propagadas en configuración TM y (b) en configuración TE. $\mathrm{C}_{g}$ representa la dirección paralela a la guía.

La distribución espacial de intensidad de la luz propagada en una guía de onda depende del campo de índice de refracción $(n)$ y de la longitud de onda $(\lambda)$ propagada. Representa el promedio en el tiempo del campo eléctrico elevado al cuadrado.

Para analizar la distribución de campo eléctrico en una guía de onda, se puede utilizar la ecuación de Helmholtz (Ecuación 1), que se obtiene partiendo de las ecuaciones de Maxwell, suponiendo que en el medio no existen cargas y que hay una dependencia del campo eléctrico armónica con el tiempo [OKA05]. Aunque la Ecuación 1, se corresponde a modos TE, para guías de bajo cambio de índice $\left(\Delta n=1 \times 10^{-2}\right)$, que es el caso analizado en el presente trabajo, los modos TE son prácticamente coincidentes con los TM [OKA05,LIN05].

Además, dicha ecuación estudia la distribución del campo eléctrico en forma escalar y no contempla la naturaleza vectorial del mismo, pero se puede utilizar como una buena aproximación para el estudio guías de onda de bajo cambio de índice. Además, tiene la ventaja de que su resolución no genera modos espurios, como ocurre en análisis vectoriales [OKA05]. 
$\nabla^{2} \varphi(x, y)+\left[k^{2} n^{2}(x, y)-\beta^{2}\right] \varphi(x, y)=0$

$\varphi(\mathrm{x}, \mathrm{y})$ es la distribución de campo eléctrico, $k$ es el número de onda $(2 \pi / \lambda)$ y $n(x, y)$ es la distribución de índice de refracción de la guía.

Existe una cantidad discreta de distribuciones de campo electromagnético que se pueden propagar en una guía de onda cada cual a una velocidad particular debido a que poseen un valor distinto de $k$. Las distribuciones de campo eléctrico, son las distintas soluciones de la ecuación de Helmholtz y se denominan modos guiados. Por ejemplo, para una guía acanalada "escalón" como la que se muestra en la Figura 4(a) se pueden obtener, resolviendo la Ecuación 1 por distintos métodos de cálculo, los modos guiados que se presentan en la Figura 4(b).

En cualquier guía de onda, el número de modos que se propague en ella y sus características dependen de: la distribución del índice de refracción; la magnitud del incremento de índice; y la longitud de onda de la radiación propagada. Al respecto, se pueden mencionar las siguientes reglas generales (obviamente, las características no mencionadas en cada ítem siguiente se asumen constantes).

- Cuando aumenta la longitud de onda, disminuye la cantidad de modos guiados

- Cuando aumentan las dimensiones de la guía, aumenta la cantidad de modos guiados

- Cuando aumenta el índice de refracción $n_{1}$ del núcleo de la guía, aumenta la cantidad de modos guiados.

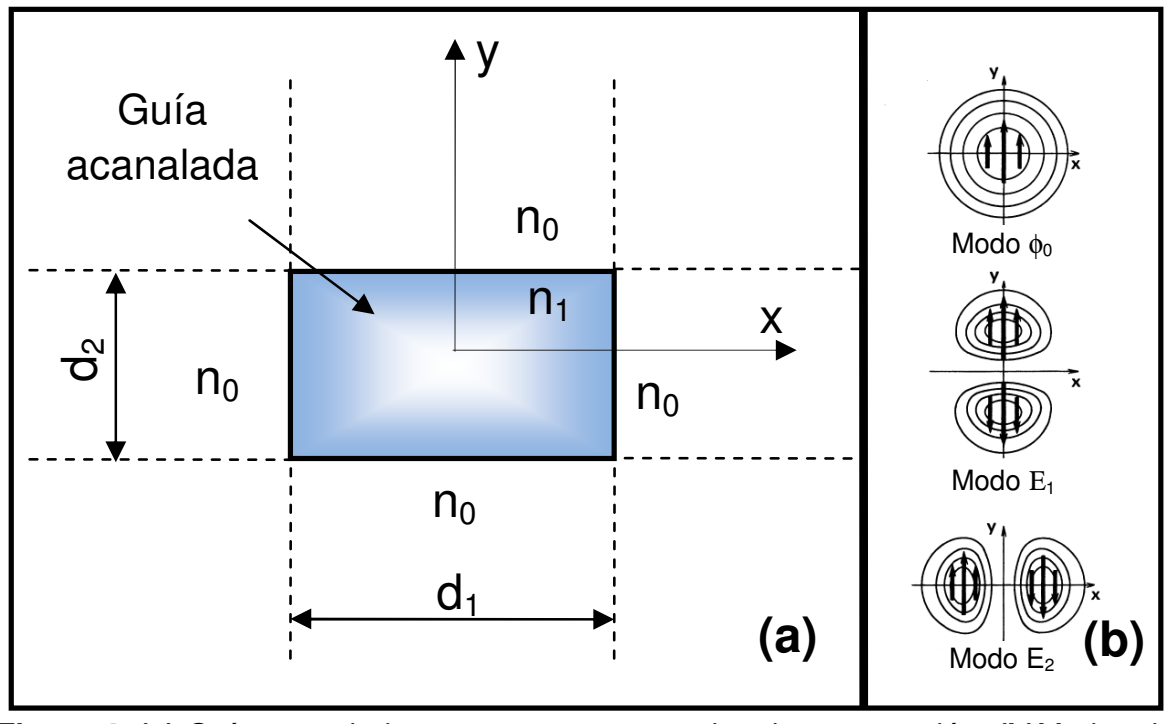

Figura 4. (a) Guía acanalada que soporta tres modos de propagación. (b)Modos de propagación $E_{0}, E_{1}$ y $E_{2}$ esquematizados con curvas de nivel de intensidad. Las flechas indican el signo de los lóbulos: estos son positivos si las flechas apuntan hacia el eje $y$ positivo y negativos en caso contrario. 
Como se mencionó anteriormente, cada uno de los modos de propagación tiene una velocidad distinta a la de los demás, por lo que no se produce interferencia entre ellos. Como consecuencia, a la salida de la guía de onda, mediante un sistema óptico con un aumento apropiado, se puede observar la suma de las distintas distribuciones de intensidad correspondientes a los modos de propagación excitados. Esta temática, está detalladamente explicada en cualquier fuente bibliográfica referida a la misma[LIN03,OKA05].

\subsubsection{Origen de guías generadas con láser de femtosegundos}

El procesado de materiales transparentes utilizando láser de pulsos ultra-cortos se basa en la absorción no-lineal originada en los materiales por la elevada intensidad de los pulsos. En general, la densidad de potencia necesaria para una interacción no-lineal suele alcanzarse solo en el volumen focal y así modificar el material de forma localizada. Por lo tanto, se puede modificar, entre otras propiedades, el índice de refracción de un material, en una región reducida. Cuando la intensidad es suficientemente alta, en general, se obtiene una fuerte disminución del índice de refracción en el volumen focal y un aumento de índice de refracción en sus adyacencias. Por lo tanto, al desplazar la muestra respecto del láser y en forma perpendicular a su propagación (Figura 5(a) y Figura 5(b)) se superponen los sucesivos volúmenes focales. Así es que, gracias a las zonas de aumento de índice (adyacentes al foco) se puede obtener el tipo de guía de onda que se estudia en este trabajo. En determinados materiales, también es posible aumentar el índice de refracción en el volumen focal con energías (intensidades) menores. Este tipo de guías pueden ser generadas en niobato de litio pero son menos estables térmicamente y no se conoce con precisión los fenómenos que la producen [BUR07]. 


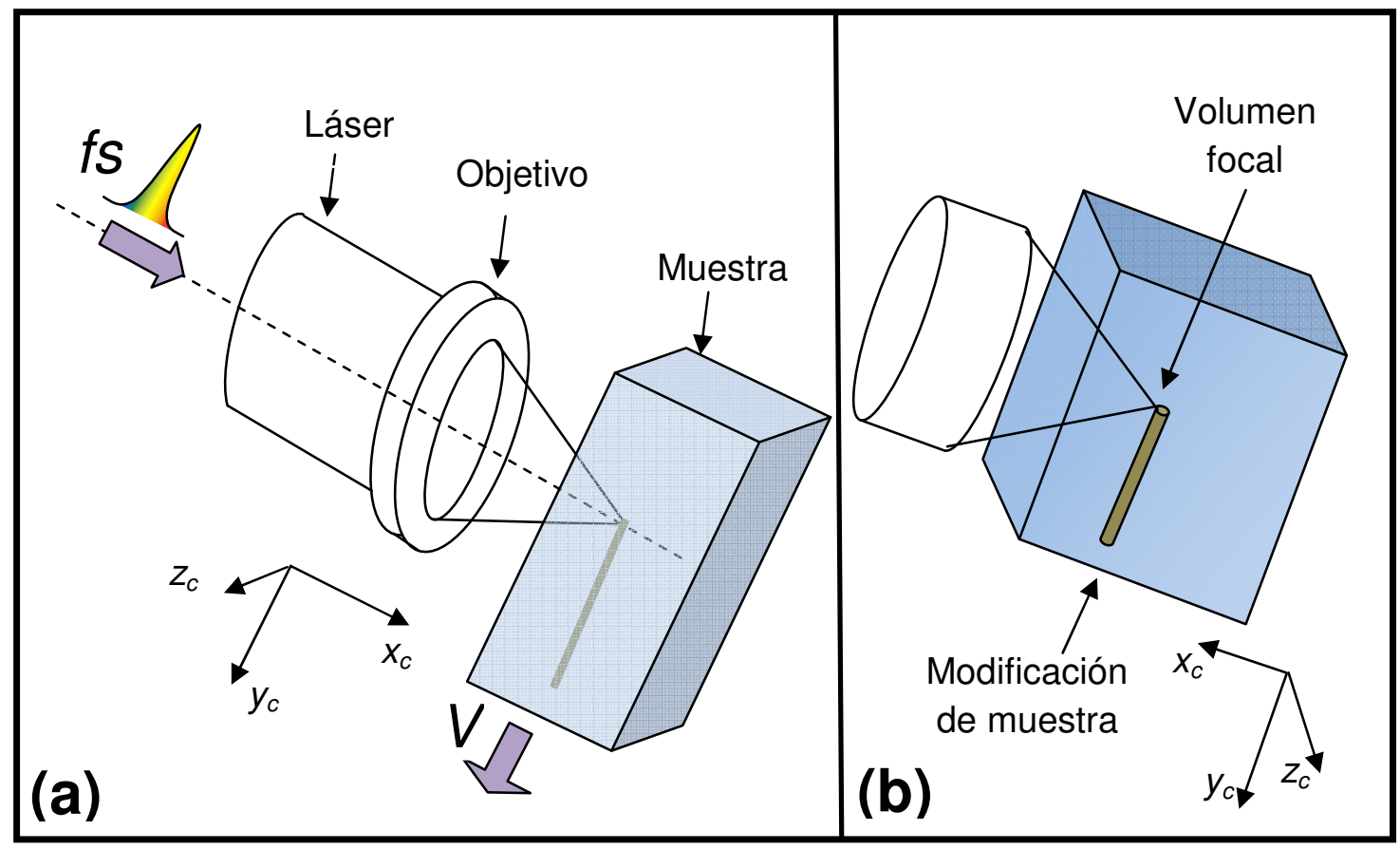

Figura 5. (a) Esquema de enfoque de láser de femto-segundos en material transparente: se indica la velocidad de desplazamiento $(V$ de la muestra. Este desplazamiento suele ser horizontal. (b) Esquema localizado del volumen focal y modificación estructural. Los ejes que se esquematizan corresponden a la estructura cristalina de $\mathrm{LiNbO}_{3}$ en la disposición utilizada para fabricar las guías estudiadas en este trabajo.

\subsection{Fenómenos generados durante y después de la interacción ultra-rápida}

Cuando se enfoca un láser de pulsos ultra-cortos (del orden de cientos de femtosegundos) en un material transparente, alcanzando altas intensidades (típicamente $10^{13}$ $\mathrm{W} / \mathrm{cm}^{2}$ ), se produce: propagación lineal y no-lineal, y, absorción lineal y no-lineal de la energía del láser por parte del material. Simultáneamente, se genera un plasma con temperaturas y presiones extremadamente altas. Luego, se produce un proceso de relajación caracterizado por ondas de choque y una re-solidificación del material [MI06].

Desde un punto de vista teórico, la descripción completa de la interacción láser-material y las modificaciones resultantes en el material, implica la resolución de las ecuaciones auto-consistentes de Maxwell del campo láser acoplado con la materia, teniendo en cuenta la distribución de energía de electrones e iones y las ecuaciones de ionización. Este problema es sumamente complejo incluso para los equipos de cómputo avanzados. De todas formas, se puede realizar un tratamiento simplificado para su comprensión, dividiendo el proceso en los siguientes sub-procesos secuenciales: propagación lineal y no-lineal, absorción lineal y no-lineal, ionización, transferencia de energía desde 
electrones a iones, conducción térmica, y expansión hidrodinámica. Sin embargo aún estudiando el problema considerando estos sub-procesos, desde un punto de vista geométrico, la complejidad del mismo perdura[MI06]. A continuación se describen brevemente los mismos.

\subsubsection{Propagación lineal}

Asumiendo una distribución de intensidad gaussiana del láser, la distribución espacial de intensidad está dada por la Ecuación 2. Las coordenadas utilizadas están en concordancia con los ejes cristalinos de la Figura 5.

$I\left(\rho, x_{c}\right)=\left(\frac{\omega_{h 0}}{\omega_{h}\left(x_{c}\right)}\right)^{2} \exp \left(\frac{-2 \rho}{\omega_{h}^{2}\left(x_{c}\right)}\right)$

Donde, $\rho$ es la coordenada polar $\left(\sqrt{y_{c}{ }^{2}+z_{c}{ }^{2}}\right)$ respecto al eje de propagación $x_{c}$ y $\omega\left(x_{c}\right)$ es el radio del haz. A su vez, $\omega_{h}\left(x_{c}\right)$ se puede definir en función de la longitud de Rayleigh $\left(\mathrm{x}_{\mathrm{c}}\right)$ y del ancho mínimo del haz $\left(\omega_{h 0}\right)$ :

$$
\omega_{h}\left(x_{c}\right)=\omega_{h 0} \sqrt{1+\left(\frac{x_{c}}{x_{c 0}}\right)^{2}}
$$

Donde, la longitud de Rayleigh está dada por:

$$
x_{c 0}=\frac{n \pi \omega_{h 0}^{2}}{\lambda}
$$

En esta ecuación, $\lambda$ es la longitud de onda del láser y $n$ es el índice de refracción del material.

Cuando este tipo de haz está colimado y es enfocado sin aberraciones, mediante una lente de apertura numérica $N A$, se genera un volumen focal elipsoide cuyos radios se definen como sigue:

$$
\begin{aligned}
& \omega_{h 0}=\frac{M^{2} \lambda}{\pi N A} \\
& x_{c 0}=\frac{M^{2} n \lambda}{\pi N A^{2}}
\end{aligned}
$$

Donde $\mathrm{M}^{2}$ es el factor de calidad de haz gaussiano. 
- Aberración esférica y refracción

Es importante tener en cuenta que cuando se enfoca un haz a través de una interface formada por medios con distintos índices de refracción, por ejemplo aire-material, ocurre un aumento de la distancia de enfoque (Figura 6(a)) por refracción, proporcional al índice de refracción del material. A su vez, los rayos que inciden en la interface con distintos ángulos, son refractados también con distintos ángulos. Esto, agrega una aberración esférica al haz y como consecuencia aumenta el radio mayor del volumen focal (Figura $6(b))$. Ambos fenómenos, son generados por la diferencia de índice de refracción entre el aire y el material.

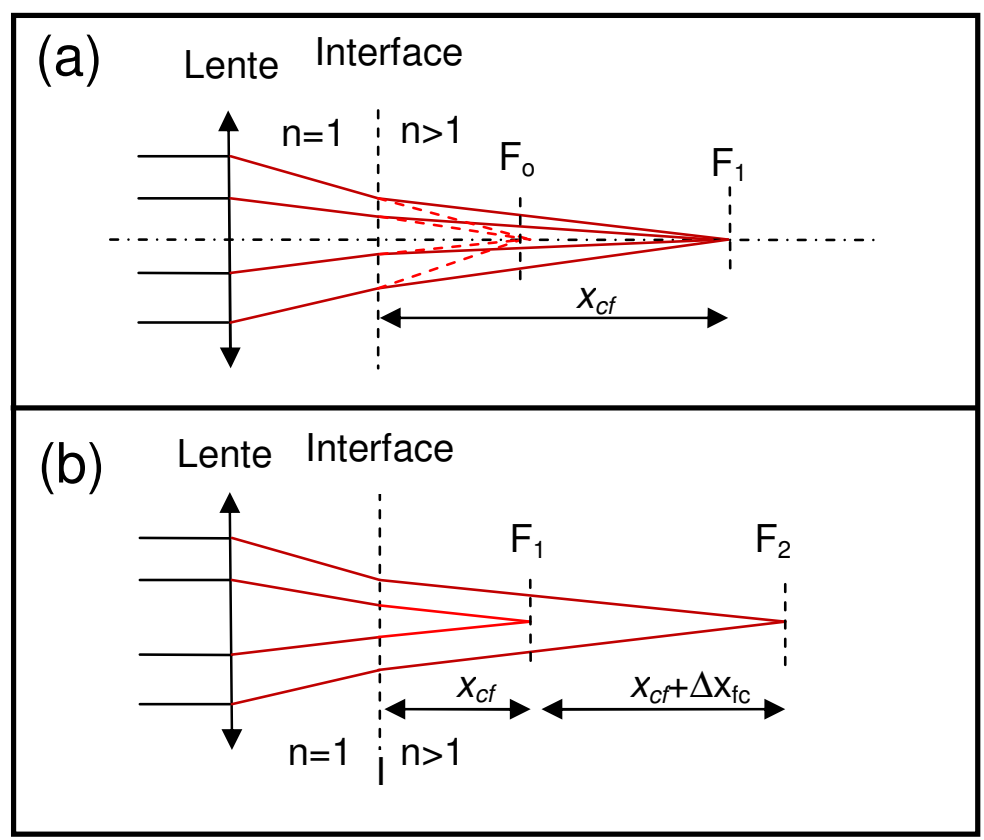

Figura 6. (a)Corrimiento del foco por la refracción sin aberración. (b)Elongación del volumen focal por aberración esférica. I: es la interface entre un material de menor índice de refracción y otro de mayor índice. $x_{c f}$ representa la profundidad del foco. La luz haz se propaga en dirección $x_{c}$.

La elongación por efecto de aberración esférica está dada, bajo aproximación paraxial, por la Ecuación 7 [MO11].

$$
\Delta x_{c f \text { esf }} \approx \frac{x_{c f}}{n} \sqrt{\frac{n^{2}-N A^{2}}{1-N A^{2}}}-n
$$

Con lo cual, al enfocar el láser dentro de un material transparente, el elipsoide determinado por los radios en las Ecuaciones 5 y 6 se ve modificado. A su vez, su radio mayor varía linealmente con la profundidad por refracción, lo que hace difícil generar tamaños de estructuras iguales a diferentes profundidades de enfoque [MO11]. 


\subsubsection{Propagación no-lineal.}

La propagación de una onda electromagnética en un medio sin cargas, está dada por la Ecuación 8.

$$
\nabla^{2} E(r, t)+\frac{n^{2} \partial^{2}}{c^{2} \partial t^{2}} E(r, t)=0
$$

Donde $r$ es el vector posición, $t$ es el tiempo y $n$ es el índice de refracción y está dado por la siguiente ecuación:

$$
n=\sqrt{1+\chi^{(1)}}
$$

Donde $\chi^{(1)}$ es la susceptibilidad de primer orden.

En régimen de propagación lineal, la polarización del medio es proporcional al campo eléctrico. En cambio, a altas intensidades, la respuesta del material deja de ser lineal y presenta anharmonicidades. Éstas, implican una dependencia no-lineal entre la polarización y el campo eléctrico. Asumiendo una dependencia instantánea entre la polarización y el campo eléctrico, la polarización se presenta en la Ecuación 10 [BO03].

$P(t)=\varepsilon_{0}\left(\chi^{(1)} E(t)+\chi^{(2)} E^{2}(t)+\chi^{(3)} E^{3}(t)+\ldots\right)$

Donde $\chi^{(2)}$ y $\chi^{(3)}$ son las susceptibilidades de orden superior y dependen de la estructura cristalina del material. Analizando vectorialmente la interacción, la primera magnitud es un tensor de segundo orden y la segunda es un tensor de tercer orden.

Para la mayoría de los materiales (amorfos o centro-simétricos) $\chi^{(2)}$ es igual a 0 . En cambio, en el caso del niobato de litio, que tiene estructura cristalina no-centrosimétrica, $\chi^{(2)}$ y $\chi^{(3)}$ son distintos de cero. En régimen no lineal, el índice de refracción se comporta como función del campo eléctrico como muestra la Ecuación 11.

$$
n=\sqrt{\frac{\varepsilon}{\varepsilon_{0}}}=\sqrt{\frac{1+\chi(E)}{\varepsilon_{0}}}
$$

Donde $\chi(E)$ recibe el nombre de susceptibilidad del medio y se define con la siguiente expresión:

$$
\chi(E)=1+\chi^{(1)} E+\chi^{(2)} E^{2}+\chi^{(3)} E^{3}+\ldots
$$


Como consecuencia de la propagación no-lineal y las aberraciones, el volumen focal abandona la forma de elipsoide, dificultando la predicción de la distribución de energía en el foco. Existen trabajos enfocados específicamente a estudiar la distribución espacial de intensidad con métodos numéricos para distintos materiales. En la Figura 7 , se presenta, a modo de ejemplo, un resultado obtenido en uno de estos trabajos [MO11] para vidrios de fosfato.

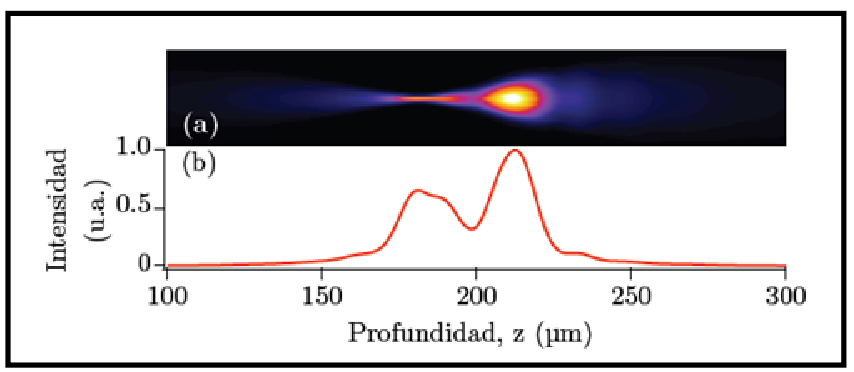

Figura 7. Simulación numérica de propagación nolineal en el foco en vidrios de fosfato. El haz incide por la izquierda. Fuente: [MO11]

- Autoenfoque no-lineal y filamentación

Como se deduce de la Ecuación 11, al propagarse un haz gaussiano de alta intensidad por un medio dieléctrico, este experimenta, (entre otros efectos [BO03]) una variación de índice de refracción que es proporcional a la intensidad de haz (considerando, de los efectos no lineales, únicamente $\chi^{(3)}$ ). De esta forma, es generado el efecto de una lente convergente, que produce el denominado autoenfoque que se esquematiza en la Figura 8.

Cuando se aumenta la potencia del haz, el efecto de autoenfoque no-lineal se vuelve más notorio hasta que alcanza la potencia crítica dada por la Ecuación 13. Es entonces, cuando se produce un equilibrio entre el autoenfoque y el desenfoque por difracción, de forma tal, que se genera un haz perfectamente colimado. Si el haz supera esta potencia, se produciría idealmente un colapso del mismo en una singularidad puntual. Sin embargo, esta teoría no es completa para tal caso, porque el haz cuando se autoenfoca puede ionizar electrones y generar un plasma. En estas condiciones, el índice de refracción es menor en el centro que en los extremos del haz produciendo un efecto de desenfoque que contrasta el efecto de autoenfoque. De esta forma, se produce un régimen de propagación en filamentación produciendo una modificación extremadamente alargada en la dirección de propagación del haz, varias veces mayor a la longitud de Raleigh del régimen lineal. Éste, no es un efecto deseable al fabricar guías de onda en la forma convencional (escritura perpendicular a la guía).

$$
P_{c r i}=\frac{3.77 \lambda^{2}}{8 \pi n n_{2}}
$$


Donde $n_{2}$ corresponde al índice de refracción no-lineal dado por la susceptibilidad de tercer orden $\left(\chi^{(3)}\right)$ [BO03].

Por lo tanto, al aumentar la energía de pulso ó al variar la profundidad de enfoque se generan modificaciones secundarias como las que se muestran en la Figura 9. En las guías estudiadas en este trabajo, se visualizó este fenómeno y se muestra en la Figura 9(b).

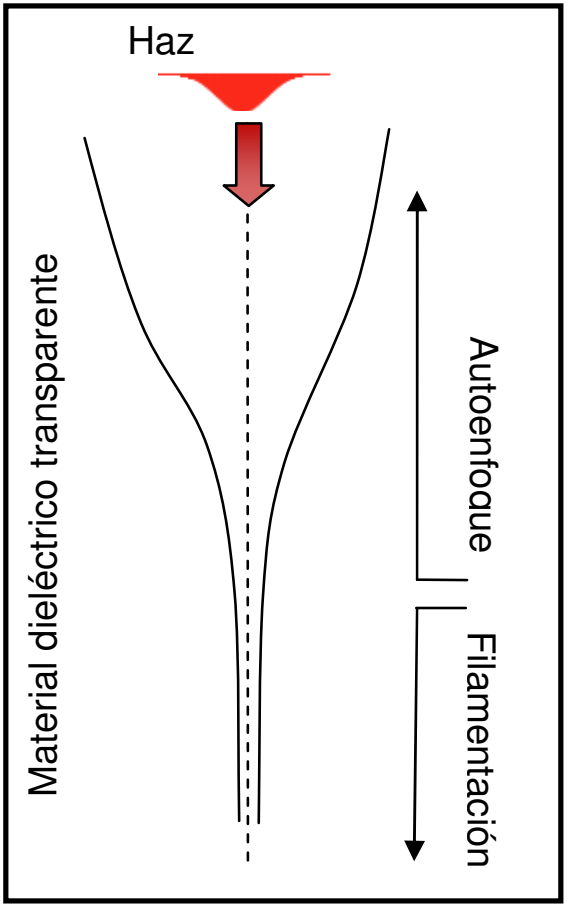

Figura 8. Esquema del autoenfoque y posterior filamentación de un haz gaussiano.

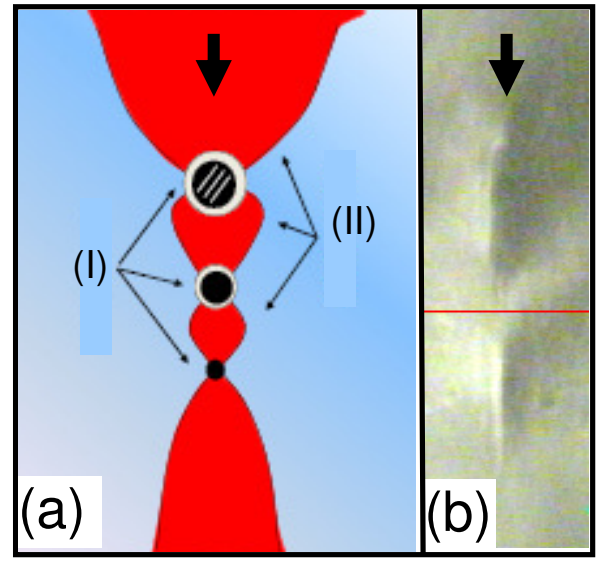

Figura 9. (a) Esquema de autoenfoque y desenfoque: (I) autoenfoque no-lineal, (II) desenfoque por generación de plasma. (b) Imagen de modificación generada con láser de femtosegundos en niobato de litio donde se observan dos zonas de modificación como consecuencia del autoenfoque del haz. La flecha superior indica la dirección de incidencia del láser

\subsubsection{Absorción de energía}

En sólidos transparentes, no se produce absorción lineal porque la energía necesaria para promover un electrón desde la banda de valencia a la de conducción (banda prohibida o bandgap) es mayor que la energía de un fotón. Este proceso, por lo tanto, no puede liberar electrones. En cambio, a intensidades suficientemente altas, un electrón puede absorber energía de múltiples fotones pudiendo así saltar la energía de la banda prohibida. Este proceso se denomina absorción multi-fotónica y depende exponencialmente de la intensidad del láser. Una vez que un electrón es promovido a la banda de conducción, este sirve como germen para promover lo que se denomina ionización por avalancha. En este proceso, este electrón puede absorber la energía del 
láser linealmente y cuando la energía absorbida supera la energía de bandgap puede transferirla a otro electrón por colisión directa para ionizarlo. Estos electrones pueden continuar el proceso dando lugar a un crecimiento exponencial de electrones libres. En estas condiciones, los electrones ionizados conforman un plasma absorbente y denso que facilita la transferencia de energía entre el láser y el material.

Cuando se generan electrones libres, se produce un estado de extremo desequilibrio donde los electrones adquieren una temperatura elevada mientras que los iones permanecen a temperatura ambiente. Esto se debe a que la escala de tiempo en la que se produce la transferencia de calor entre los electrones y la estructura iónica es mucho mayor que la duración del pulso. Por lo tanto, los electrones y los iones tienen que ser descriptos con diferentes temperaturas. Para estudiar y caracterizar este fenómeno, fue propuesto el modelo de dos temperaturas [ANI74] representado en las siguientes ecuaciones:

$$
\begin{aligned}
& C_{e} \cdot \frac{\partial T_{e}}{\partial t}=-\frac{\partial Q(z)}{\partial z}-\gamma \cdot\left(T_{e}-T_{i}\right)+S \\
& C_{i} \cdot \frac{\partial T_{i}}{\partial t}=\gamma \cdot\left(T_{e}-T_{i}\right)
\end{aligned}
$$

Donde $T_{e}$ y $T_{i}$ son las temperaturas de electrones y iones y $z$ es la dirección perpendicular a la superficie de incidencia. $C_{e}$ y $C_{i}$ son las capacidades caloríficas del sistema de electrones y de los iones. El parámetro $\gamma$ caracteriza el acoplamiento electróniones, es decir el intercambio de energía entre ambos sistemas. $Q(z)$ es el flujo de calor y $S$ es el término correspondiente a la fuente de calor del láser.

La transferencia de energía entre electrones e iones en un plasma a alta temperatura ocurre en el orden de picosegundos. La temperatura máxima $T_{e}$ que pueden alcanzar los electrones es cercana a 250 ev ó $3 \mathrm{MK}$, dependiendo del material y del láser, y su correspondiente presión es del orden de 25 Mbar. Por lo tanto, el sólido es transformado en un estado de gas ideal, pero manteniendo la densidad del sólido [MI06]. Esto genera fuertes condiciones de desequilibrio las que hacen dificultoso un análisis detallado teórico debido a la rápida y significativa variación de las distintas propiedades representadas en la Ecuación 15 (capacidad calorífica, conductividad térmica, tiempo de relajación, reflectividad y coeficiente de absorción).

\subsubsection{Expansión/relajación}

Una vez producida la transferencia completa de energía entre electrones e iones, como consecuencia de la absorción de energía por parte del material, comienza un movimiento hidrodinámico. Emerge una onda térmica y una onda de choque que se propaga desde el volumen focal hacia el seno del material. Esta última puede alcanzar una presión del orden de los $100 \mathrm{GPa}$, con lo cual el material es modificado de forma 
permanente, generando una nueva estructura, en general y en particular en niobato de litio, de menor densidad promedio que el material sin modificar. La generación de esta nueva estructura está vinculada con las deformaciones residuales que se generan en los alrededores de la zona modificada, una vez finalizado el proceso de relajación [MI06].

Como resultado de esta interacción, en materiales dieléctricos transparentes, se obtiene una zona central de fuerte modificación estructural claramente delimitada (donde cambian sus propiedades mecánicas y ópticas) y una zona lindante con deformaciones residuales ó modificaciones estructurales de menor envergadura [RO08].

Cabe mencionar que si bien se conoce el orden de magnitud de las variables de estado (presión, temperatura y volumen) durante los procesos mencionados, no se conocen con exactitud las características de los mismos. Estas incluyen, por hacer mención de algunas: la relación entre el láser enfocado y la real distribución de densidad de energía absorbida, la densidad de electrones generada, la distribución de temperatura y presión y las características geométricas de la expansión hidrodinámica. Tampoco se conoce la geometría real de la cavidad que se forma, ni la fase exacta de la misma [MI06, OSE12].

\subsection{Deformaciones y tensiones en sólidos deformables. Efecto piezo-óptico}

\subsubsection{Deformaciones y tensiones en sólidos deformables}

Cuando se aplican fuerzas en un sólido, este se deforma cambiando su forma y volumen. En la Teoría de Elasticidad del Continuo, la posición de cada punto de un sólido es descrito por un vector $r$ de tres componentes $r_{1}, r_{2}, r_{3}$. La posición de cada uno de estos puntos después de deformarse se puede llamar $\mathbf{r}^{\prime}$. Entonces se define el desplazamiento u como la diferencia entre estos vectores:

$u=r^{3}-r$

Los vectores desplazamientos de los distintos puntos del sólido definen completamente el estado mecánico del sólido deformado. Antes de la deformación la distancia entre estos puntos $(d l)$ es:

$$
d l^{2}=d x^{2}+d y^{2}+d z^{2}
$$

Después de la deformación $d x^{\prime}=d x+d u$ (lo mismo ocurre para las direcciones $y$ y $z$ ), entonces la nueva distancia entre estos puntos $(d l)$ es: 


$$
d l^{\prime 2}=d x^{2}+d y^{\prime 2}+d z^{\prime 2}
$$

Reemplazando $d x^{\prime}, d y^{\prime} y d z^{\prime}$ como función de $d u$, reordenando e introduciendo un tensor de segundo orden, se pueden obtener las siguientes ecuaciones [CHA07]:

$$
d l^{2}=d l^{2}+2 \varepsilon_{i k} d x_{i} d x_{k}
$$

$\varepsilon_{i k}=\frac{1}{2}\left(\frac{\partial u_{i}}{\partial x_{k}}+\frac{\partial u_{k}}{\partial x_{i}}+\frac{\partial u_{l}}{\partial x_{i}} \frac{\partial u_{l}}{\partial x_{k}}\right)$

Donde $\varepsilon_{i k}$ se denomina tensor de deformaciones $\mathrm{y}$ es un tensor de segundo orden $\mathrm{y}$ simétrico, y $d x_{i}$ y $d x_{k}$ representan $d x\left(d x_{1}\right), d y\left(d x_{2}\right)$ y $d z\left(d x_{3}\right)$.

Cuando un sólido se deforma por aplicación de fuerzas externas $(F)$ se rompe el equilibrio y aparecen fuerzas internas que intentan devolver al sólido el estado inicial. Estas fuerzas internas por unidad de área se denominan tensiones mecánicas.

Bajo la formulación débil [CHA07], las tensiones son iguales a la fuerza aplicada por unidad de área. Por lo tanto es válida la siguiente igualdad:

$$
\int F d v=\oint \sigma_{i k} d f_{k}
$$

Donde $\sigma_{\mathrm{ik}}$ son las tensiones internas en el material.

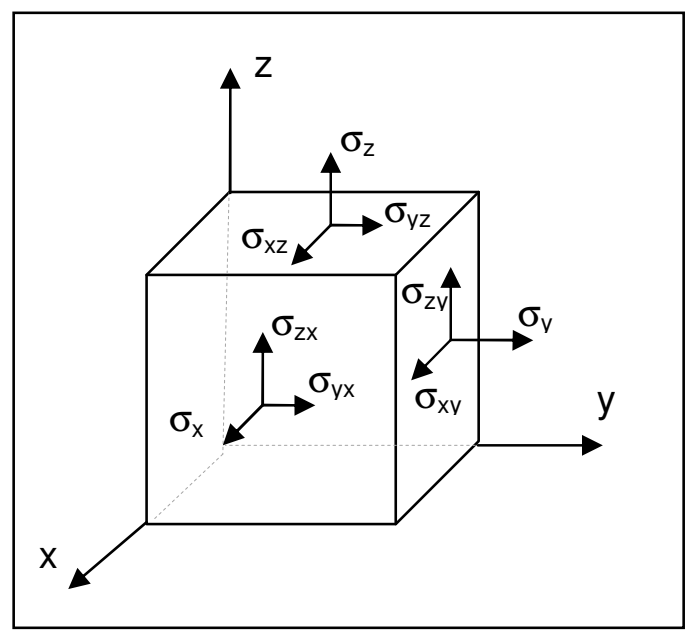

Figura 10. Esquema de tensiones mecánicas en un infinitesimal de sólido.

En consecuencia, se puede asociar un tensor de segundo orden ó una matriz de tensiones a un infinitesimal de sólido deformado como se muestra en la Figura 10. En la teoría de deformación lineal, la relación entre las deformaciones y las tensiones 
originadas en el sólido es lineal. Esta relación se muestra en la Ecuación 22, en su forma matricial.

$$
\left[\begin{array}{c}
\sigma_{x} \\
\sigma_{y} \\
\sigma_{z} \\
\sigma_{y z} \\
\sigma_{x z} \\
\sigma_{x y}
\end{array}\right]=\left[C_{i j}\right] \cdot\left[\begin{array}{c}
\varepsilon_{x} \\
\varepsilon_{y} \\
\varepsilon_{z} \\
\varepsilon_{y z} \\
\varepsilon_{x z} \\
\varepsilon_{x y}
\end{array}\right]
$$

Donde la matriz $\left[C_{i j}\right.$ ] se asume constante para un determinado material y se denomina matriz constitutiva del mismo. Ésta, es una matriz de $6 \times 6$ y describe su comportamiento en régimen lineal. A su vez, se puede demostrar que cumple las condiciones completas de simetría[TI96]. Por lo tanto, contiene 21 elementos independientes como máximo. De acuerdo a los ejes y planos de simetría que posea la estructura de un material, los elementos independientes van reduciendo su número. Los casos extremos son los materiales triclínicos y los isotrópicos. Los denominados triclínicos no poseen ninguna simetría de rotación ni plano de simetría. En cambio, los isotrópicos poseen infinitos planos y ejes de simetría. Como consecuencia, los materiales triclínicos poseen 21 elementos independientes y los isotrópicos poseen 2 elementos independientes en sus matrices constitutivas. Las restantes clases principales de materiales son: Monoclínicos, Ortotrópicos, Trigonales, Tetragonales, Isotrópico Transversal y Cúbicos. Estas poseen, en su matriz [ $\left.C_{\mathrm{ij}}\right], 13,9,6,6,5$ y 3 elementos independientes respectivamente.

A continuación se presenta la forma de la matriz de elasticidad de los materiales trigonales (Ecuación 23). Estos tienen tres planos de simetría: uno a 0ํy los otros dos a $\pm 60^{\circ}$.

$$
[C i j]=\left[\begin{array}{cccccc}
C_{11} & C_{12} & C_{13} & C_{14} & 0 & 0 \\
& C_{11} & C_{13} & -C_{14} & 0 & 0 \\
& & C_{33} & 0 & 0 & 0 \\
& & & C_{44} & 0 & 0 \\
& & & & C_{44} & C_{14} \\
& & & & & \frac{C_{11}-C_{12}}{2}
\end{array}\right]
$$

Para el niobato de litio, los valores de $C_{i j}$ en $\mathrm{Pa}$, son los siguientes [WO02]:

$C_{11}=2.05 \times 10^{11} ; C_{12}=0.57 \times 10^{11} ; C_{13}=0.70 \times 10^{11} ; C_{14}=0.079 \times 10^{11} ; C_{33}=2.49 \times 10^{11}$; 


$$
\mathrm{C}_{44}=0.609 \times 10^{11} ; \mathrm{C}_{66}=(\mathrm{C} 11-\mathrm{C} 12) \times 0.5=0.75 \times 10^{11} ;
$$

Esta relación también se puede expresar en la siguiente forma:

$$
\left[\begin{array}{c}
\varepsilon_{x} \\
\varepsilon_{y} \\
\varepsilon_{z} \\
\varepsilon_{x y} \\
\varepsilon_{x z} \\
\varepsilon_{y z}
\end{array}\right]=\left[S_{i j}\right] \cdot\left[\begin{array}{c}
\sigma_{x} \\
\sigma_{y} \\
\sigma_{z} \\
\sigma_{x y} \\
\sigma_{x z} \\
\sigma_{y z}
\end{array}\right]
$$

Donde $\left[S_{i j}\right]$ es igual a la inversa de la matriz $\left[C_{i j}\right]$.

Asumiendo un comportamiento elástico lineal, las deformaciones y tensiones dentro de un sólido deformable pueden estimarse utilizando la teoría de elasticidad lineal para una determinada condición de carga. La misma propone la combinación de diversas ecuaciones diferenciales en derivadas parciales que incluyen: equilibrio interno del sólido, equilibrio externo y condiciones de borde, ecuación constitutiva del material y ecuaciones cinemáticas [SAN]. Estas ecuaciones se analizan en el Capítulo 3 para el caso bidimensional. Unificando las mismas, se puede obtener la Ecuación de Navier, para materiales isotrópicos presentada en la Ecuación 25.

$$
\left(\lambda_{L}+G\right) \operatorname{grad}(\operatorname{div}(u))+G \nabla^{2}(u)+X=0
$$

Donde $\lambda_{L}$ es el módulo de Lamé y $G$ es el módulo de cizalladora, ambas constantes definidas a partir del comportamiento elástico del material isotrópico [SAN], $u$ es el campo de desplazamientos y $X$ son las fuerzas de volumen.

$\mathrm{Si}$ el campo de desplazamientos interno del sólido satisface la Ecuación 25 y las condiciones de contorno (desplazamientos preestablecidos o cargas aplicadas), entonces ese campo de desplazamiento es solución del problema elástico. A partir del mismo, se pueden obtener, los campos de deformaciones y tensiones dentro del sólido [SAN].

Para resolver por el método de elementos finitos el campo de desplazamientos $u$ se utiliza comúnmente la formulación débil, que es una forma integral de la Ecuación 25. Esta Formulación se muestra en su forma tensorial y para materiales isotrópicos, en la Ecuación 26 [BO12].

$$
\int_{\Omega} \frac{\mu_{L}}{2}\left(\nabla u+\nabla u^{T}\right) \cdot\left(\nabla v+\nabla v^{T}\right) d \Omega+\int_{\Omega} \lambda(\nabla \cdot u)(\nabla \cdot v) d \Omega=\int_{\Omega} v \cdot X d \Omega+\int_{\Gamma_{N}} v \cdot \hat{t} d \Gamma
$$


Donde $\mu_{L}$ y $\lambda_{\mathrm{L}}$ son las constantes de Lamé, que dependen del comportamiento elástico del material isótropo, $v$ es una función de prueba, $X$ representa las fuerzas de volumen y $\boldsymbol{t}$ las condiciones de borde, $\Omega$ es el dominio del sólido elástico y $\Gamma_{\mathrm{N}}$ es el borde del mismo. Esta formulación variacional no exige a la solución (campo de desplazamientos $u$ ) derivadas de orden superior continuas

Aunque la Ecuación 26 corresponde a materiales isotrópicos, se estudiará en el Capítulo 4 como se aplica su análoga a materiales ortotrópicos, después de realizada la reducción denominada deformaciones planas.

\subsubsection{Efecto piezo-óptico}

Cuando en un material se producen deformaciones, éstas generan variaciones en las componentes del tensor de índice de refracción debido al efecto piezo-óptico. Despreciando términos de orden superior, esta variación está dada por la siguiente expresión tensorial [RO09_2]:

$$
\Delta n_{i}=-\frac{1}{2}\left(n_{i}\right)^{3} \cdot \sum_{j} p_{i j} \varepsilon_{j}
$$

Donde $p_{\mathrm{ij}}$, son los coeficientes piezo-ópticos propios del material, $\varepsilon_{j}$ representa las componentes del tensor de deformaciones con nomenclatura reducida de Voigt [TI96] $\left(\varepsilon_{1}=\varepsilon_{\mathrm{xc}}, \varepsilon_{2}=\varepsilon_{\mathrm{yc}}, \varepsilon_{3}=\varepsilon_{\mathrm{zc}}, \varepsilon_{3}=\varepsilon_{\mathrm{zc}}, \varepsilon_{4}=\varepsilon_{y z c}, \varepsilon_{5}=\varepsilon_{x z c}\right.$ y $\left.\varepsilon_{6}=\varepsilon_{x y c}\right)$. Para cristales de niobato de litio, se midieron los valores de $\mathrm{p}_{\mathrm{ij}}$ presentados en la Tabla $\mathrm{i}$ [WO02]. Los mismos fueron utilizados en todos los cálculos del presente trabajo.

\begin{tabular}{|c|c|c|c|c|c|c|c|c|}
\hline & $p_{11}$ & $p_{12}$ & $p_{13}$ & $p_{14}$ & $p_{31}$ & $p_{33}$ & $p_{41}$ & $p_{44}$ \\
\hline $\mathrm{LiNbO}_{3}$ & -0.026 & 0.09 & 0.133 & -0.075 & 0.179 & 0.071 & -0.151 & 0.146 \\
\hline
\end{tabular}

Tabla i. Valores de constantes piezo-ópticas de niobato de litio.

Se ha reportado[MY09], que el dopaje de Mg no afecta de manera relevante a los valores de $p_{\mathrm{ij}}$, por este motivo se utilizaron los valores presentados en la Tabla i para el niobato de litio puro y para aquel dopado con $\mathrm{Nd:Mg.}$ 



\section{Instrumental, técnicas experimentales y materiales empleados}

En este capítulo se describen las técnicas experimentales utilizadas durante el trabajo de tesis. A su vez, se explican los fundamentos de la espectroscopía Raman y su aplicación particular al $\mathrm{LiNbO}_{3}$. Se mencionan también las propiedades y aplicaciones relevantes de este cristal. Finalmente, se presenta la Teoría de Deformación Potencial Lineal y los resultados de estudios Raman bajo deformaciones mecánicas en niobato de litio reportados previamente.

\subsection{Láser de femtosegundos}

Las guías de onda estudiadas en el presente trabajo se fabricaron utilizando un Láser de femtosegundos de Titanio-Zafiro de la firma Spectra-Physics (USA). El mismo, consta de dos subsistemas, uno denominado "oscilador" (Tsunami, Spectra-Physics) y otro denominado "amplificador" (Spitfire, Spectra-Physics). En conjunto, el sistema es capaz de emitir pulsos a una longitud de onda central de $800 \mathrm{~nm}$ con una duración de 150 fs y con una tasa de repetición de $1 \mathrm{KHz}$. La energía máxima disponible es de $0.8 \mathrm{~mJ}$ por pulso. El sistema denominado CPA (Chirped Pulse Amplifier), se muestra en la Figura 11 y su modo de operación es bien conocido desde finales de la década de 1980 [STRI85, MAR86]. 


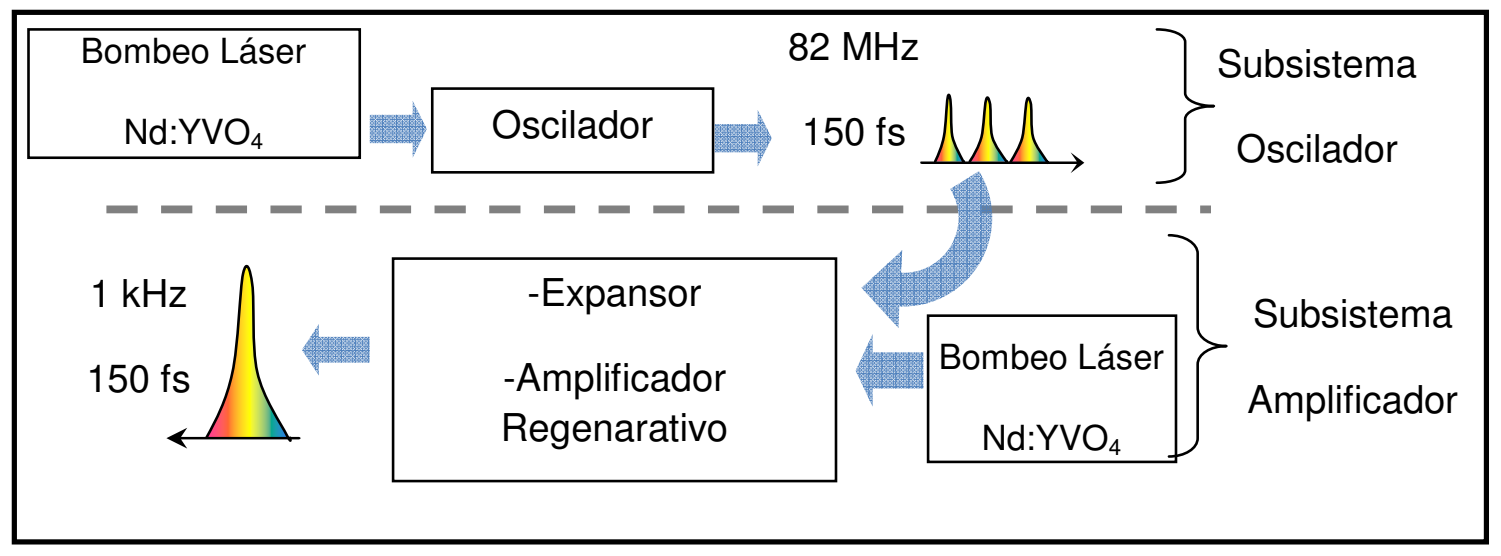

Figura 11. Sistema de generación de láser de femtosegundos

El oscilador se basa en el método de anclado de modos [FLE86,FER03] (Kerr lens mode locking) para generar pulsos láser a partir de un medio activo Ti:Zafiro y el bombeo de un láser continuo de $1064 \mathrm{~nm}$ doblado a $532 \mathrm{~nm}$ y 4W. Este subsistema genera pulsos de una duración de 150 fs con una energía de 9nJ y una tasa de repetición de $82 \mathrm{MHz}$ (Figura 11). Estos pulsos son sintonizables en longitud de onda. En el presente trabajo, la misma se fijó en $800 \mathrm{~nm}$ con un ancho espectral aproximado de $9 \mathrm{~nm}$.

El tren de pulsos obtenido del oscilador se introduce en el amplificador, donde primeramente, los pulsos son expandidos temporalmente mediante un conjunto de redes de difracción para disminuir la potencia pico y no dañar los componentes ópticos al ser amplificados. Luego, el tren de pulsos es introducido en el amplificador regenerativo donde se utiliza un medio activo de Ti:Zafiro bombeado con un láser de estado sólido $\left(\mathrm{Nd}: \mathrm{YVO}_{4}\right)$. En este subsistema, los pulsos expandidos recorren varios ciclos de amplificación hasta que son liberados por acción de una célula pockels en combinación con un polarizador de lámina delgada.

Una vez amplificados los pulsos, el diámetro del haz es aumentado a $7.5 \mathrm{~mm}$ para evitar daños en los componentes ópticos y se introducen en la etapa de compresión. Esta comprende un montaje similar al de la etapa de expansión pero inverso, para obtener pulsos de $150 \mathrm{fs}$ a una tasa de repetición de $1 \mathrm{KHz}$ y a una energía de $0.8 \mathrm{~mJ}$.

\subsubsection{Regulación de potencia óptica}

Para el mecanizado de materiales con un láser de femtosegundos, un parámetro fundamental es la energía de pulso. Las modificaciones estructurales que se originen en el material dependen fuertemente de la misma. Dicha energía es controlada mediante una lámina de media onda $(\lambda / 2)$ y un polarizador. La radiación a la salida del amplificador esta polarizada de forma horizontal. La disposición de esta lámina $\lambda / 2$ y del polarizador se presenta en el esquema de la Figura 12. Con la lámina $\lambda / 2$ se gira la polarización del láser y cuando éste se encuentra con el polarizador, sólo pueden atravesarlo las 
componentes que se encuentran orientadas en dirección vertical. Después de atravesar el polarizador, la potencia del haz es medida utilizando un medidor de potencia óptica Spectra Physics modelo 407 A que posee una resolución 1mW [LAB02]. La potencia media en función de la energía de pulso está dada por la siguiente expresión:

$$
\bar{P}=\frac{\left(e_{p} \cdot n_{p}\right)}{\Delta t}
$$

Donde: $P$ es la potencia media en un intervalo de tiempo $\Delta \mathrm{t}, e_{\mathrm{p}}$ es la energía por pulso y $n_{\mathrm{p}}$ es la cantidad de pulsos en el intervalo $\Delta \mathrm{t}$.

Teniendo en cuenta que la tasa de repetición es de $1 \mathrm{kHz}$ (1000 pulsos por segundo) y considerando $\Delta \mathrm{t}=1 \mathrm{~s}$, podemos obtener la energía de pulso en función de la potencia medida:

$$
e_{p}=\frac{\bar{P}}{1000}
$$

Por lo tanto, la mínima energía detectable en forma directa corresponde a $1 \mu \mathrm{J}$. Como se requiere fijar valores de energía de pulso menores a $1 \mu \mathrm{J}$, se colocó un atenuador óptico neutro de $10 \mathrm{~dB}$ previamente caracterizado (Figura 12).

\subsubsection{Estación de micro-mecanizado.}

Las guías de onda estudiadas en este trabajo, se fabricaron en configuración transversal. En la misma, el haz de femtosegundos permanece fijo e incide en forma perpendicular a la dirección de desplazamiento de la muestra.

Para realizar el proceso de escritura, el láser de femtosegundos es reflejado hasta llegar a una estación de micro-mecanizado mediante una serie de espejos, como se observa en la Figura 12. De esta forma, llega hasta un objetivo de microscopio que lo enfoca en la muestra en dirección perpendicular al plano xy de la estación de micro-mecanizado. En este trabajo, se estudiaron guías fabricadas con un objetivo Edmun de 20X (NA 0.4, DIN 20) a pupila llena y con otro objetivo Olympus de 10 X (NA 0.3). 


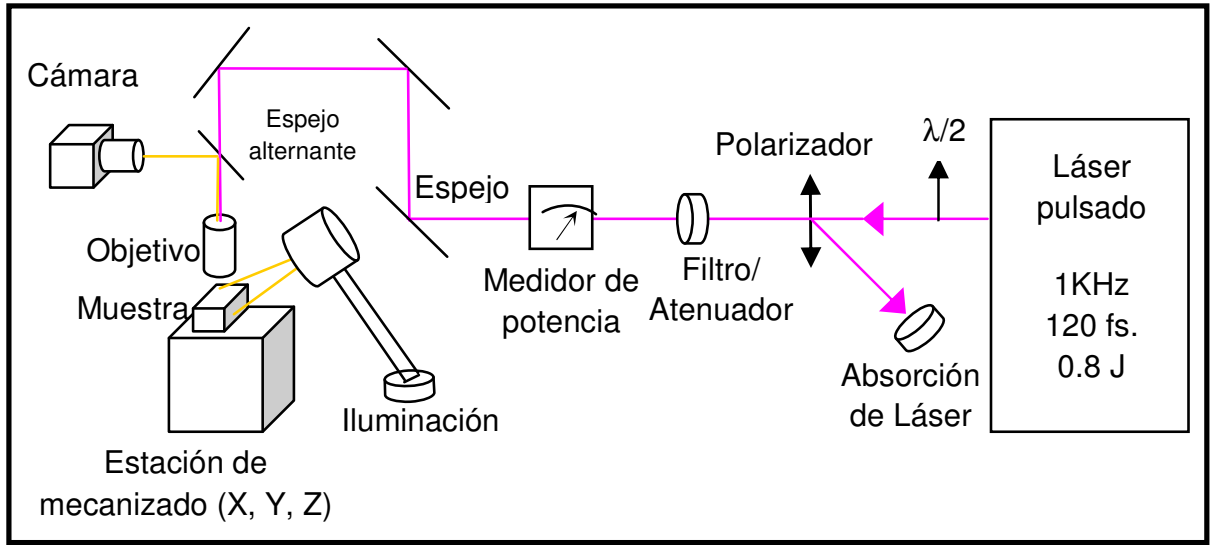

Figura 12. Esquema de sistema láser y estación de micro-mecanizado

A su vez, un espejo alternante, una fuente de luz blanca y una cámara convencional permiten visualizar la superficie e interior de la muestra. Esta última, se coloca sobre un conjunto de posicionadores Newport controlados por software. Estos posicionadores (Figura 13), permiten desplazarla controlando posición y velocidad en tres ejes perpendiculares entre sí (X, Y, Z). La resolución espacial de estos motores es de $0.05 \mu \mathrm{m}$ y el rango es de $5 \mathrm{~cm}$.

La velocidad de desplazamiento utilizada para fabricar las guías es de $25 \mu \mathrm{m} / \mathrm{s}$ en todos los casos del presente trabajo. La dirección de enfoque es la del eje cristalino $x_{c}$, y la dirección de desplazamiento de la muestra coincide con el eje cristalino $\mathrm{y}_{\mathrm{c}}$, para todos los casos estudiados en este trabajo.

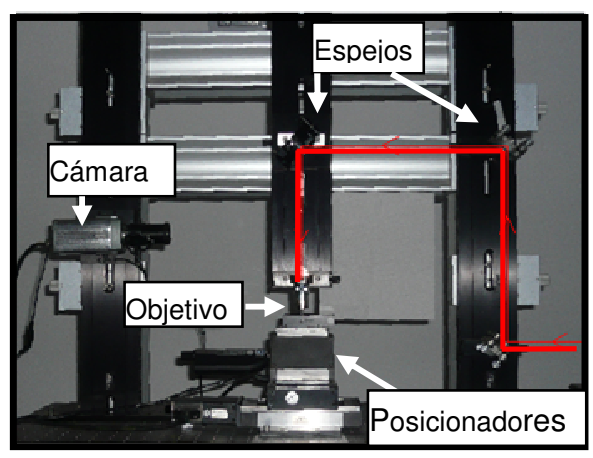

Figura 13. Imagen del sistema de micro-mecanizado

\subsection{Acople de luz en guías de onda.}

Una vez generadas las guías de onda con escritura láser, estas pueden ser acopladas con luz láser convencional para identificarlas y caracterizarlas. Para esto, se utilizó el método conocido como "End-Fire", que permite acoplar luz láser en dichas guías [RO09]. 
En la Figura 14 se muestra un esquema del sistema utilizado. Todos los posicionadores se encuentran solidarios a una mesa óptica.

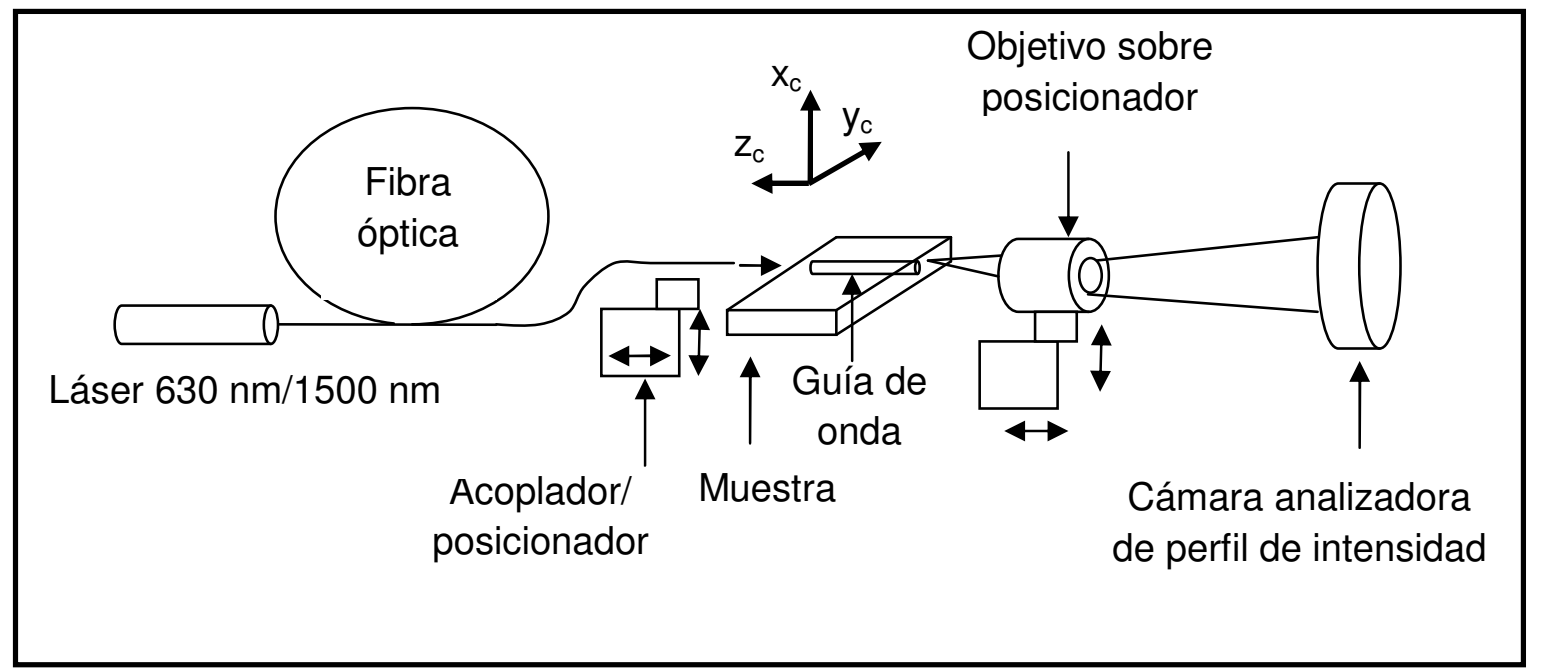

Figura 14. Esquema de arreglo experimental para acoplar luz láser a guías de onda

El sistema está conformado por los siguientes componentes (Figura 14):

- un láser de $630 \mathrm{~nm}$ y otro de $1500 \mathrm{~nm}$ que alternadamente se pueden acoplar a un tramo de fibra óptica

- un tramo de fibra óptica con un extremo conectado a un láser y el otro se encuentra solidario a un acoplador de fibra óptica, que permite desplazar micrométricamente la fibra óptica en tres direcciones ( $X, Y$ y $Z$ )

- un posicionador micrométrico manual sobre el que se coloca la muestra con una sola dirección de posicionamiento (dirección $\mathrm{z}_{\mathrm{c}}$ en Figura 15(a)).

- un objetivo de microscopio (10 $\mathrm{x})$ sobre un posicionador micrométrico manual con tres direcciones de desplazamiento $(X, Y$ y $Z$ ) que se utiliza para hacer imagen de la salida de las guías de onda.

- un dispositivo analizador de perfiles de intensidad (Newport LBP-4 de 8 bits) solidario a la mesa óptica y ubicado a $65 \mathrm{~cm}$ del objetivo. 


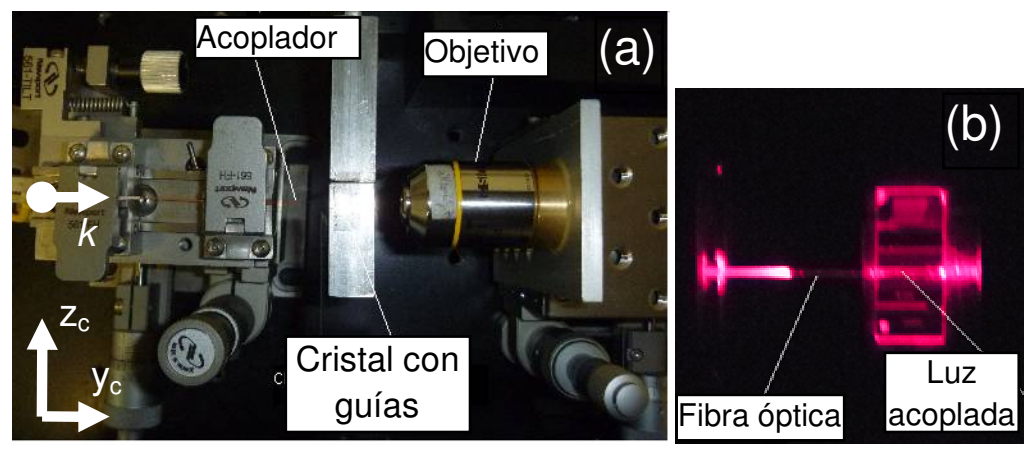

Figura 15. (a) Imagen de sub-sistema acoplador-cristal-objetivo. Se indica la dirección $k$ de la luz incidente, los ejes presentados corresponden a la estructura cristalina del material utilizado, (b) imagen de láser de $650 \mathrm{~nm}$ acoplado en guía de onda.

\subsubsection{Procedimiento para acople "End-fire"}

Para acoplar las guías de onda se alinearon de forma manual los posicionadores de cada componente. Primeramente, se enfoca la cara posterior del cristal desplazando manualmente el posicionador que posee el objetivo de 10x y utilizando como referencia un arista del cristal. Esto se realiza iluminando con el láser de $650 \mathrm{~nm}$ mediante la fibra óptica dispuesta a una distancia aproximada de $2 \mathrm{~mm}$ de la cara anterior del cristal. La imagen de la sección transversal del cristal se visualiza a simple vista sobre una superficie blanca a una distancia lejana del objetivo (1m aproximadamente). Desplazando el cristal mediante su posicionador, se pueden visualizar las modificaciones estructurales generadas con el láser de femtosegundos. Luego, se aproxima la fibra óptica a la cara anterior del cristal hasta observar "puntos" de luz muy intensos en las adyacencias de las modificaciones. Éstos, son formados por la luz guiada en las estructuras.

Una vez acopladas las guías en las adyacencias de las estructuras, se coloca la cámara analizadora de perfiles de intensidad y mediante el software provisto por el fabricante, se puede registrar una imagen de la intensidad guiada en las estructuras estudiadas. En la Figura 16 se muestra un ejemplo de una guía acoplada, la elipse blanca representa la ubicación de la modificación estructural. La escala de la imagen fue obtenida a partir de la visualización de estructuras de longitud conocida. 


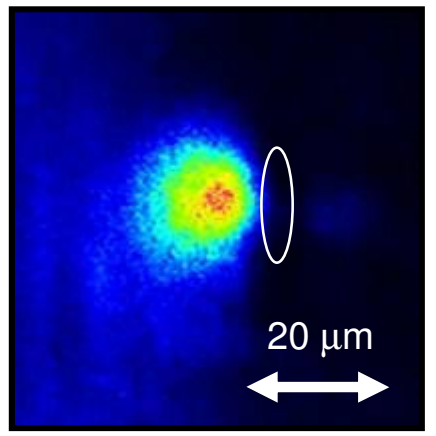

Figura 16. Imagen de láser acoplado en una guía de onda fabricada con láser de femtosegundos. La elipse representa la modificación estructural.

\subsection{Espectroscopia Raman en niobato de litio}

La espectroscopia Raman concierne el estudio de la luz dispersada por interactuar con los modos de vibración propios de la estructura del material a nivel interatómico. En la Figura 17(a) se puede ver un esquema del fenómeno. Cuando la luz impacta en una estructura atómica, ésta sufre una momentánea distorsión en sus electrones. De esta forma, la luz incidente genera un momento dipolar inducido en las moléculas constitutivas del material. Éste, es precedido por una re-emisión al relajarse las moléculas. Entonces, es cuando se emiten solo algunos fotones con frecuencia diferente a la de la luz incidente (Dispersión Raman). La diferencia entre la energía de los fotones emitidos y la de los fotones incidentes se denomina corrimiento Raman. 


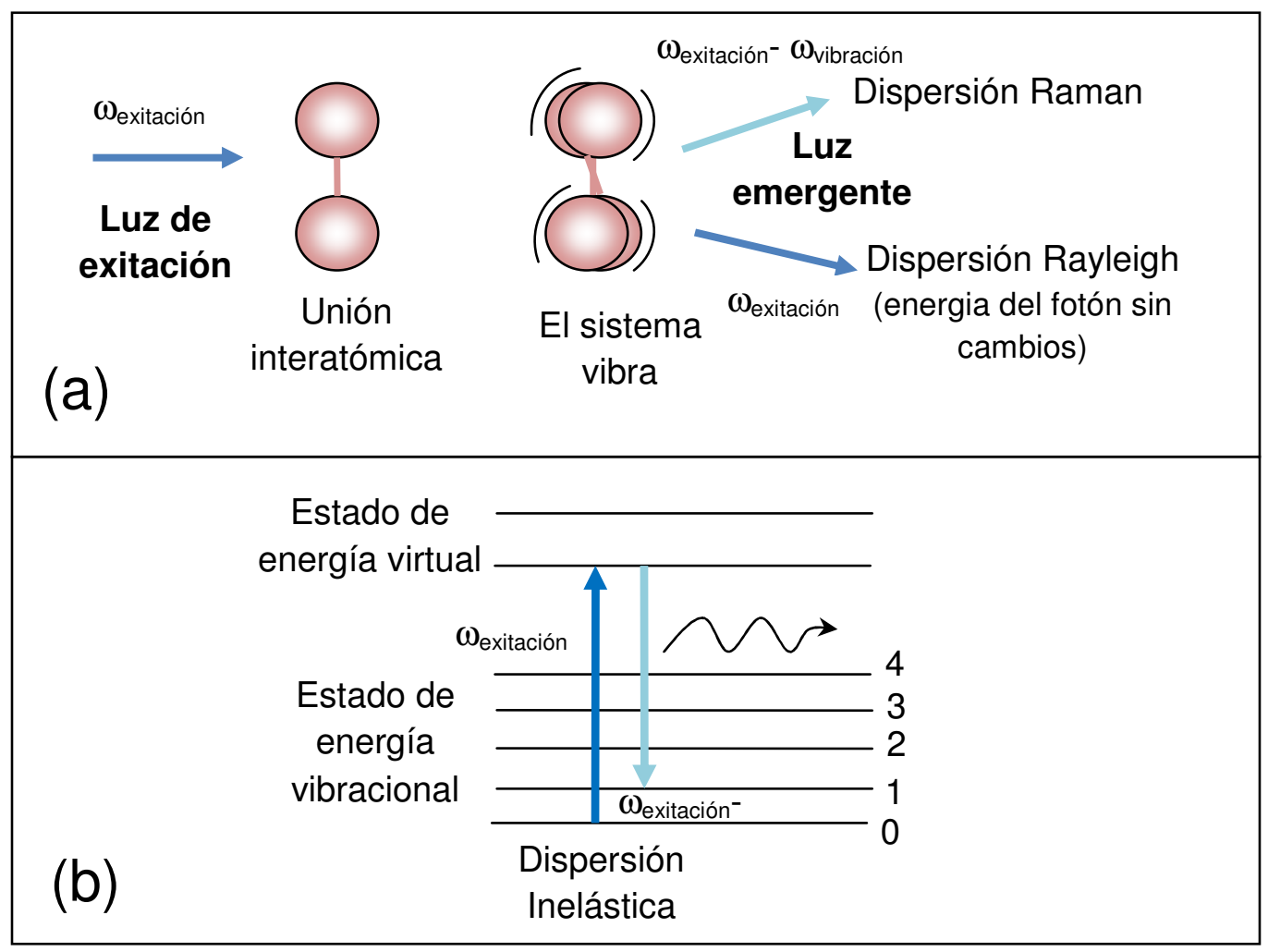

Figura 17. (a) Esquema de fenómeno de dispersión Raman. (b) Estados de energía que transita una estructura atómica durante este fenómeno.

Determinados modos de vibración asociados a una estructura cristalina pueden generar distintos corrimientos Raman. A su vez, la frecuencia de vibración de estos modos es directamente proporcional al corrimiento Raman, definida por la siguiente expresión.

$\Delta e=h v_{f}$

Donde $\Delta e$ es la diferencia de energía entre el fonón Raman y el láser incidente, $v_{f}$ es la frecuencia de vibración propia de la estructura atómica y $h$ es la constante de Planck.

Como consecuencia, en un espectro Raman se ven caracterizadas frecuencias de vibración de la estructura cristalina, con lo cual, conforma una suerte de huella digital de dicha estructura. Los fotones dispersados por efecto Raman se denominan fonones.

En general, se utiliza luz láser para excitar este proceso porque su eficiencia es extremadamente baja $(0.001 \%)$. En la Figura 18, se muestran espectros Raman del niobato de litio.

\section{- Notación de Porto}

Por otro lado, como esta espectroscopia tiene origen en los dipolos inducidos en el material, los fonones excitados, en el caso de materiales de estructuras ordenadas, dependen de la combinación de: $\left(D_{1}\right)$ la dirección incidente del laser respecto de la celda cristalina, $\left(D_{2}\right)$ la dirección de la polarización con que incide el láser, $\left(D_{3}\right)$ la dirección de 
la polarización de la luz que es dispersada y colectada, y $\left(D_{4}\right)$ la dirección en que es medida esta última. Estas direcciones se indican respecto a los ejes de la estructura del material. Comúnmente se utiliza la llamada notación de Porto que se expresa de la forma: $D_{1}\left(D_{2} D_{3}\right) D_{4}$, donde $D_{i}$ son las direcciones mencionadas. Las distintas combinaciones de estas direcciones reciben el nombre de "configuraciones". Por ejemplo, en el niobato de litio $\left(\mathrm{LiNbO}_{3}\right)$ se pueden medir espectros Raman distintos para distintas configuraciones, en la Figura 18 se muestran dos configuraciones $(y(x)) \tilde{y}$ y y $(z$ :)ỹ) de este cristal. El signo ":" indica que la polarización colectada ó de salida, no ha sido seleccionada. En ellas, se pueden observar que los fonones excitados en cada caso son distintos y se ajustan, en general, a funciones lorentzianas.
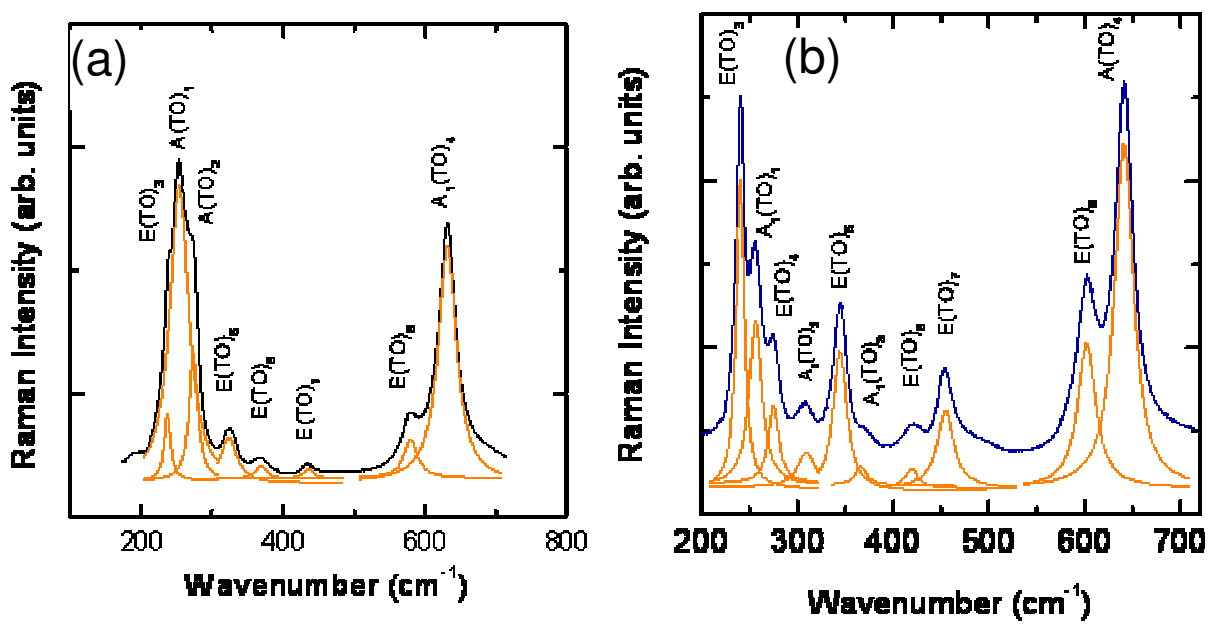

Figura 18. Espectro Raman de niobato de litio dopado con magnesio. (a)Configuración y(zx)ỹy $+y(z z) \tilde{y}$. (b)Configuración y $(x x) \tilde{y}+y(x z) \tilde{y}$.

\subsubsection{Mapeos de microscopia Raman}

En este trabajo se presentan mapeos micro-Raman unidimensionales y bidimensionales de diferentes guías de onda para estudiar las deformaciones residuales que las conforman. Para generar estos mapas, se requiere colectar un espectro en cada punto o pixel del mapa, almacenarlo y luego procesar el conjunto de espectros.

Para la exploración micro-Raman se utilizó un sistema estándar para tal fin disponible en la Universidad Autónoma de Madrid. En la Figura 19 se muestra un esquema del mismo.

El láser utilizado para la excitación es de Argón (514.2 nm) con potencia máxima de 200 mW. Para enfocar el láser, se utilizó un microscopio Confocal Olympus BX-51 con un objetivo de microscopio de 50 X (NA=0.75).

Todos los mapeos se realizaron $10 \mu \mathrm{m}$ por debajo de la superficie en configuración "back-scattering", es decir que, la luz incidente tiene la misma dirección que la luz 
colectada pero distinto sentido. El láser incide en la muestra en la dirección $\mathrm{y}_{\mathrm{c}}$ del cristal y está polarizado en la dirección $z_{c}$ del mismo. La luz colectada atraviesa un filtro estrecho "notch" centrado en $514.2 \mathrm{~nm}$ para remover del espectro, el láser de excitación, $y$, un polarizador orientado en dirección $z_{c}$ para seleccionar los fonones de la configuración Raman requerida: $\mathrm{Y}(\mathrm{ZZ}) \mathrm{Y}$.

Para la colección del espectro Raman, se utilizó un doble Monocromador con una red de difracción de 1800 líneas/mm y una cámara detectora Horiba refrigerada con nitrógeno liquido con una temperatura de trabajo de $-65^{\circ} \mathrm{C}$. También se utilizó un sistema de posicionamiento asistido por computadora con una resolución de $0.1 \mu \mathrm{m}$.

El sistema completo es controlado por el software comercial LabSpec®, que permite realizar espectros en distintos puntos de la muestra en forma automatizada con tiempos de muestro pre-establecidos, promediar varias medidas por punto y almacenarlas. Los tiempos de muestreo de los espectros fue de $3 \mathrm{~s}$ y en cada punto el espectro fue promediado 3 veces. Con estos parámetros, en el espectro obtenido en cada punto, se obtuvo una relación señal-ruido máxima de aproximadamente 10000.

Para realizar el procesamiento de los espectros Raman colectados y obtener los corrimientos espectrales de los fonones ó picos, también se utilizó el software LabSpec®. Este permite filtrar y ajustar los miles de espectros correspondientes a los diferentes puntos del mapeo de forma automatizada. El ajuste de los picos se realizó utilizando una función Lorentziana para los fonones $A_{1}(T O)$.

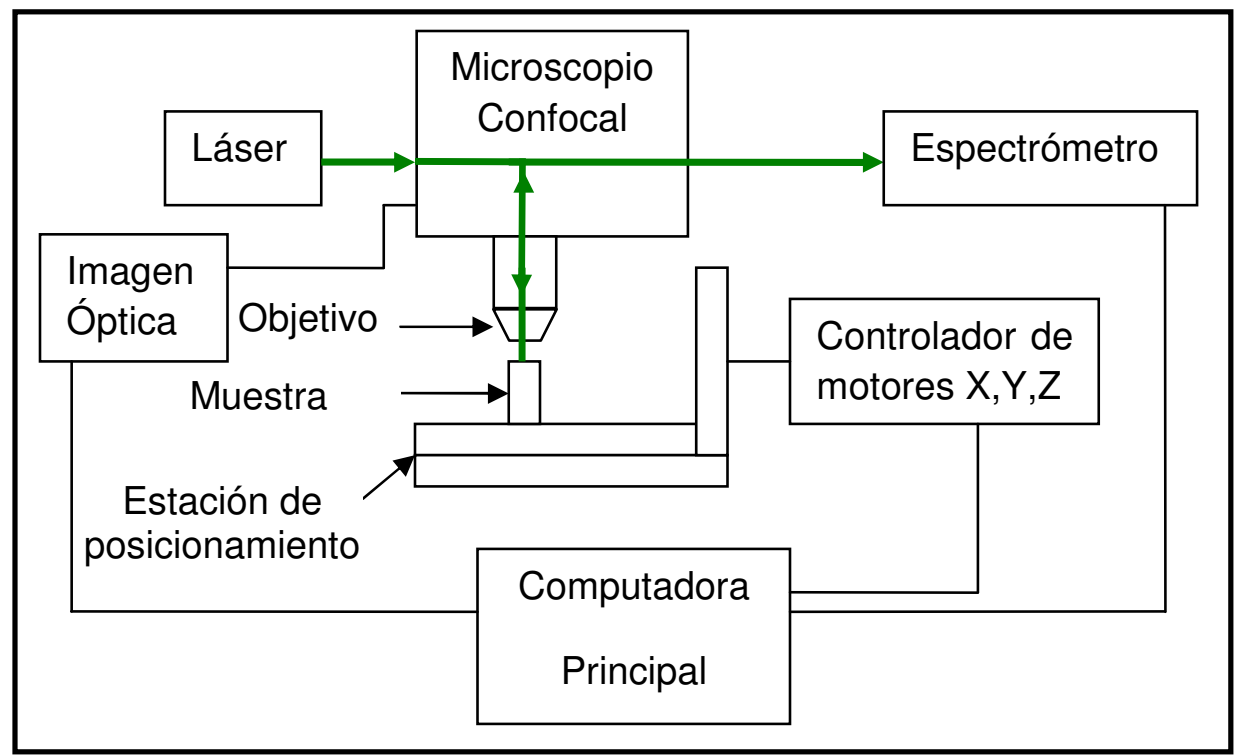

Figura 19. Esquema de Sistema de Microscopía Raman 


\subsection{Espectro Raman de cristales deformados: Teoría de Deformación Potencial Lineal (TDPL)}

La Teoría de Deformación Potencial Lineal relaciona las deformaciones existentes en la red cristalina con las variaciones en el espectro Raman por intermedio de coeficientes lineales. En particular, para el caso de los fonones de simetría $A_{1}$, el corrimiento espectral respecto de su posición característica, en presencia de deformaciones y despreciando términos de orden superior, está dado por:

$\Delta \omega_{A 1(T O) i}=e_{i}\left(\varepsilon_{x c}+\varepsilon_{y c}\right)+f_{i}\left(\varepsilon_{z c}\right)$

Donde $\Delta \omega$ es el corrimiento en número de onda del fonón $A_{1}(T O)_{i}$ respecto de su posición nominal, $e_{i}$ y $f_{i}$ son las constantes de deformación potencial (propias del material) y $\varepsilon_{\mathrm{xc}}, \varepsilon_{\mathrm{yc}}$ y $\varepsilon_{\mathrm{zc}}$ son los elementos diagonales del tensor de deformaciones (ó componentes normales) respecto de los ejes cristalinos. Cada fonón $A_{1}(T O)_{i}$ tiene, en general, distintos valores de $e_{i}$ y $f_{i}$.

Para analizar un caso en el que se aplique presión hidrostática, es conveniente reemplazar en la ecuación anterior, las deformaciones por tensiones, utilizando la relación constitutiva (Ecuación 22) y llegando a la Ecuación 32.

$$
\Delta \omega_{A 1(T O) i}=e_{i}^{\prime}\left(\sigma_{x c}+\sigma_{y c}\right)+{f^{\prime}}_{i}\left(\sigma_{z c}\right)
$$

Donde $e_{i}^{\prime}$ y $f_{i}^{\prime}$ están dadas por:

$$
e_{i}^{\prime}=e_{i}\left(S_{11}+S_{12}\right)+f_{i}\left(S_{12}\right)
$$

$f^{\prime}{ }_{i}=2 e_{i} S_{12}+f_{i}\left(S_{33}\right)$

En el caso de aplicar al material una presión hidrostática $p$ que lo comprima y considerando la Ecuación 21, $\sigma_{\mathrm{xc}}=\sigma_{\mathrm{yc}}=\sigma_{\mathrm{zc}}=-p$. Entonces la Ecuación 32 queda:

$$
\Delta \omega_{A 1(T O) i}=e_{i}^{\prime}(-2 p)+f_{i}^{\prime}(-p)
$$

Dividiendo ambos miembros entre $p$ :

$$
\frac{\Delta \omega_{A 1(T O) i}}{\Delta p}=-\left(2 e_{i}^{\prime}+f_{i}^{\prime}\right)
$$

El cociente de la Ecuación 36 representa el coeficiente de presión hidrostática que se suele medir experimentalmente. De aquí en adelante $d \omega / d p$. 


\subsection{Sistema de celda de diamantes para compresión hidrostática y sistema de colección de espectro Raman.}

En esta tesis se presentan medidas bajo presión hidrostática del espectro Raman del $\mathrm{Nd}: \mathrm{Mg}: \mathrm{LiNbO}_{3}$ realizadas por el Dr. A. R. Goñi y K. Pereira da Silva en el Instituto de Materiales de la Universidad Autónoma de Barcelona. A continuación se describe el equipamiento utilizado.

\subsubsection{Sistema de aplicación de presiones.}

El sistema utilizado para realizar esta experiencia es del tipo "Syassen-Holzapfel" [JAY83], se esquematiza en la Figura 20(a) y está compuesto por los siguientes subsistemas:

- Una celda de diamante para compresión: consiste en dos diamantes de geometría cónica truncada que permiten ejercer altas presiones deformando un anillo de metal (gasket) sin verse afectados por deformaciones permanentes. A su vez, como son transparentes permiten utilizar métodos ópticos para medir características de una muestra colocada en la cámara que se forma entre ellos. Se presenta un esquema en la Figura 20(b).

- Un anillo de metal con un orificio de aproximadamente $200 \mu \mathrm{m}$ de diámetro ("gasket") que genera una cámara donde se debe depositar la muestra que se somete a presión. Además, se coloca una mezcla de metanol etanol (4:1) como medio trasmisor de la presión hidrostática dentro de la cámara. Se presenta un esquema en la Figura 20(b).

- Como elemento testigo, para medir la presión dentro de la cámara se colocó un pequeño trozo de silicio dentro de la misma. La espectroscopia Raman del silicio está bien caracterizada bajo presión hidrostática [QIN01] y no se superpone con el espectro del niobato de litio. Esta metodología, se utilizó en reemplazo de la bien conocida técnica de luminiscencia de rubí para evitar errores espectrales asociados al desplazamiento del monocromador, requerido para medir la posición del pico de luminiscencia del rubí en cada etapa de medición. En la Figura 20(b) se presenta un esquema de la cámara.

- Para comprimir los diamantes se utilizó el mecanismo presentado en la figura Figura 20(a). El pistón superior es comprimido simultáneamente por dos brazos. Estos son girados desde su parte inferior por varillas roscadas accionadas por un conjunto de engranajes. 


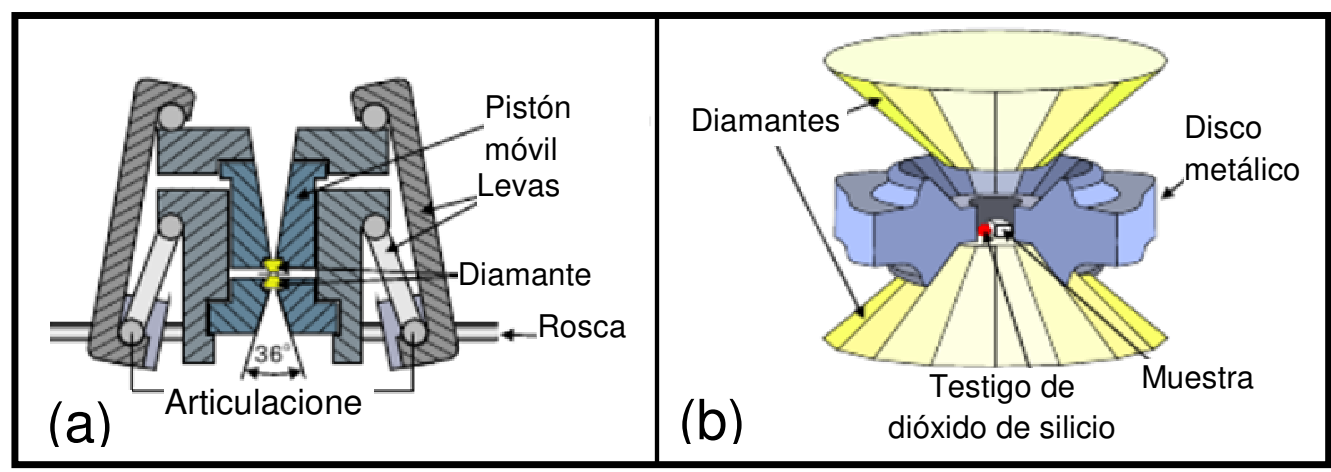

Figura 20. (a) Esquema de sistema mecánico que comprime el conjunto de diamantesanillo (b) Diamantes y anillo metálico (gasket)

\subsubsection{Equipamiento Raman para estudio bajo presión hidrostática.}

Los espectros Raman fueron colectados en la configuración back-scattering con un espectrómetro de alta resolución LabRam HR800[LAB03], utilizando una red de difracción de 1800 líneas/mm y un Dispositivo Detector de Carga Acoplada (CCD) refrigerado por nitrógeno líquido. Para la excitación se utilizó un láser de Argón de $514.6 \mathrm{~nm}$ que fue enfocado dentro de la muestra con un objetivo Olympus de larga distancia de trabajo de 20x. La polarización del láser incidente se orientó utilizando una lámina $\lambda / 2$ en la dirección $x_{c} y$ en la dirección $z_{c}$ del cristal. Todas las mediciones se realizaron a temperatura ambiente.

\subsubsection{Estudios previos de Raman en niobato de litio bajo presión hidrostática}

En la bibliografía existente al comenzar la presente investigación, existía escasa información experimental del comportamiento del espectro Raman del niobato de litio en presencia de deformaciones. Solo se encontraron trabajos que datan de la década de 1980[JAY86, ME84]. En ellos, se realizaron estudios de la espectroscopia mencionada en niobato de litio bajo presión hidrostática hasta altas presiones (0 a 20 Gpa) y de a intervalos de 0.1 Gpa aproximadamente. Aunque las deformaciones mecánicas a estudiar no se producen bajo presión hidrostática y se estipulan que alcanzan valores que rondan los 0.4 Gpa [BUR07], esta información resultó valiosa al comienzo del presente trabajo, porque se pueden relacionar los corrimientos bajo presión hidrostática con aquellos bajo deformaciones "no hidrostáticas" mediante la Ecuación 36. Los corrimientos de los fonones bajo presión hidrostática, en ambos trabajos citados, parecen seguir un comportamiento lineal hasta los $6 \mathrm{Gpa}$. En la Tabla ii se presentan los valores de variación de número de onda en función de la presión para los fonones $A_{1}(T O)$ del niobato de litio. 


\begin{tabular}{|c|c|c|}
\hline & \multicolumn{2}{|c|}{$d \omega / d p$ (cm-1/ Gpa) } \\
\hline Fonón & $\begin{array}{c}\text { Mendes-Filho[Fl84] } \\
\text { (0 a 8 GPa) }\end{array}$ & $\begin{array}{c}\text { Jayaraman[JAY86] } \\
\text { (0 a 20 GPa) }\end{array}$ \\
\hline$A_{1}(\mathrm{TO})_{1}$ & 0.0 & 2 \\
\hline $\mathrm{A}_{1}(\mathrm{TO})_{2}$ & 5 & 6 \\
\hline $\mathrm{A}_{1}(\mathrm{TO})_{3}$ & 2 & 6 \\
\hline $\mathrm{A}_{1}(\mathrm{TO})_{4}$ & 1.5 & 2 \\
\hline
\end{tabular}

Tabla ii. Corrimiento en número de onda de los fonones del niobato de litio respecto de su posición característica en función de presión hidrostática.

Los fonones $A_{1}(T O)$ se utilizarán en la caracterización porque tienen comportamiento fácil de cuantificar en presencia de deformaciones no-hidrostáticas (Ecuación 31) y son fácilmente separables de los restantes fonones (ver Figura 21(c) ).

Se puede ver en la Tabla ii, que para ambos estudios, los coeficientes de presión de los fonones $A_{1}(T O)_{i}$ tiene el mismo orden de magnitud pero no coinciden sus valores. Lamentablemente, en las mismas no se ha realizado un análisis comparativo ni mención alguna sobre esta discrepancia. Podrían influir diversos factores (se mencionan en orden de mayor a menor influencia esperada): rango de presiones, forma de medición y composición de cristal. No se profundizará sobre este tema. En este trabajo se utilizarán los valores medidos por Mendes-Filho porque el rango presiones estudiado se encuentra más cercano al estudiado en este trabajo y porque, a diferencia de Jayaraman, presenta las incertezas correspondientes a cada coeficiente de presión $d \omega / d p$ hallado.

Por consiguiente, se puede decir que el niobato de litio no se encontraba caracterizado "piezo-espectroscópicamente" para deformaciones generalizadas al comenzar este trabajo ya que no se conocían los valores de las constantes e y $f$ (Ecuación 31), ni la relación entre ellos. Solo estaba caracterizado bajo presión hidrostática y con algunas discrepancias entre los reportes mencionados.

Para estudiar el comportamiento del espectro Raman del niobato de litio bajo deformaciones, también podrían hacerse simulaciones desde el punto de la física básica de la interacción radiación-materia utilizando por ejemplo el método "ab-initio" [VA01,SA13], pero esto escapa al alcance de este Trabajo de Tesis. Además, los resultados que se obtienen de estas técnicas para este cristal, no son exactos ya que resultan de aplicar numerosas aproximaciones [VA01]. 


\subsection{Materiales: niobato de litio puro $\left(\mathrm{LiNbO}_{3}\right)$ y dopado con magnesio y neodimio (Mg:Nd:LiNbO}

En este trabajo de tesis se utilizan dos muestras de niobato de litio, donde se fabrican las guías de onda estudiadas. Una de ellas, dopada con neodimio $(0.3 \%)$ y magnesio $(5 \%)$ y la otra sin dopantes. La primera, fue fabricada en la Universidad Autónoma de Madrid por el método Czochralski[PA06], alcanzando una composición congruente. Sus dimensiones son de 10x1 x3 mm. La muestra de niobato de litio sin dopantes fue suministrada por el fabricante SAES Getters $\AA$, su composición también es congruente y su dimensiones son de 40x10x1 mm. En ambas muestras, el eje cristalino $x_{c}$ es normal a su máxima superficie (Figura 21(d)). Este tipo de muestra se denomina "corte-x".

El niobato de litio, es un material ferroeléctrico propicio para una gran variedad de aplicaciones opticas/fotónicas. Esto se debe a sus excelentes propiedades electroópticas, no lineales y piezo-eléctricas, entre otras. Es un material ampliamente caracterizado y las tecnicas de crecimiento artificial lo pueden producir de gran tamaño (del orden de algunos centímetros) y de alta calidad. Este material, es un cristal de estructura trigonal, del grupo $3 \mathrm{~m}$, que se crece artificialmente bajo atmosfera controlada, su mínima estructura posee 5 atomos: 1 de Litio, 1 de Niobio y 3 de Oxigeno. Por ejemplo, en espectroscopia Raman, para la configuración $Y(Z Z) X$ se excitan únicamente los fonones correspondientes a la simetría $A_{1}$, este espectro se muestra en la Figura 21(c) [VA01]. 


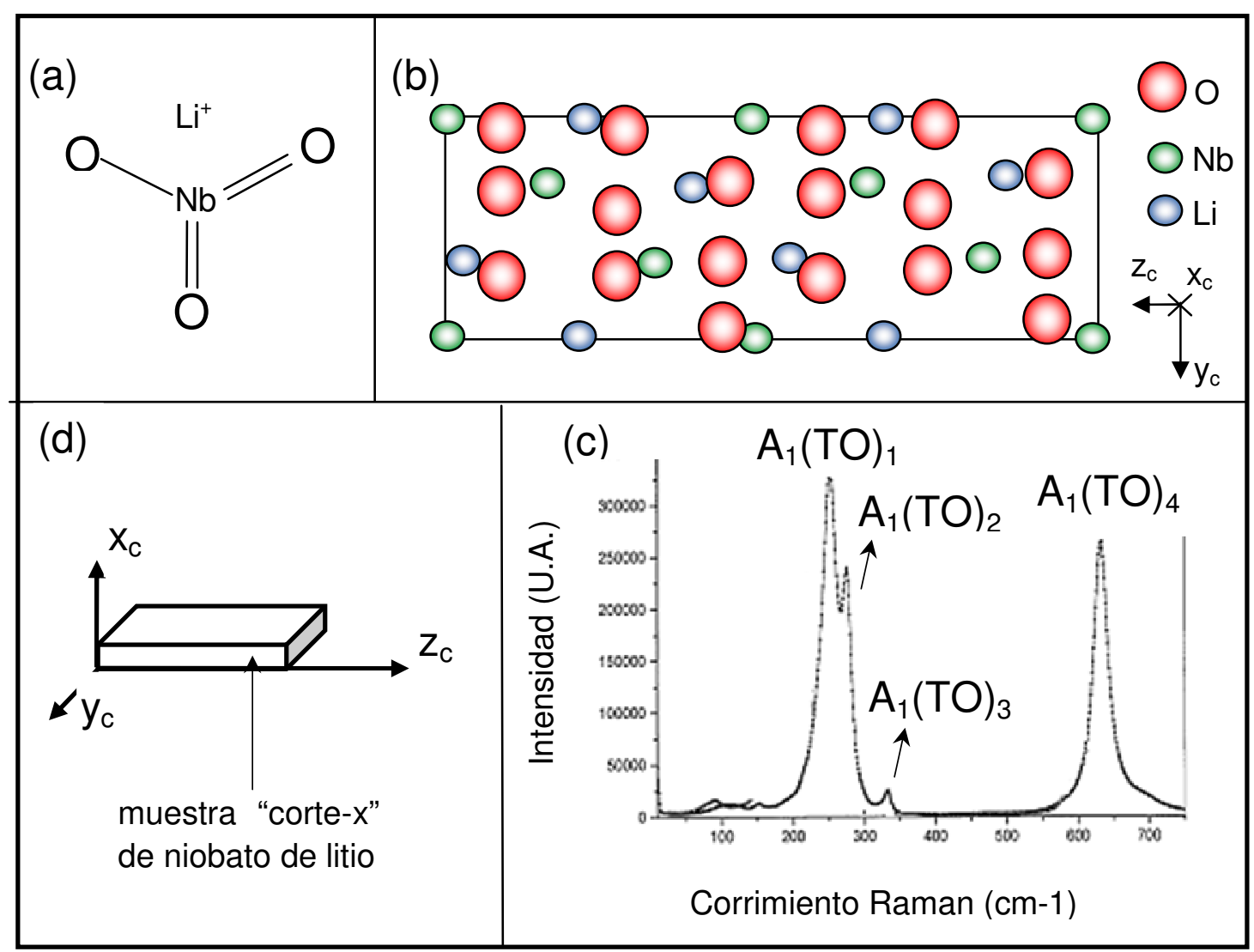

Figura 21. (a) Notación química del niobato de litio, (b)esquema de celda unidad de niobato de litio $\left(\mathrm{LiNbO}_{3}\right)$ vista desde el eje $\mathrm{x}$, (c) espectro Raman de $\mathrm{LiNbO}_{3}$ en configuración $\mathrm{x}(\mathrm{ZZ}) \mathrm{x}$, (d) esquema de muestras utilizadas

El dopaje con metales de transición, principalmente con Magnesio (Mg), se utiliza para aumentar la resistencia al daño foto-refractivo del material. Es decir, que incrementa su capacidad de resistir potencia óptica. Esto, es deseable principalmente cuando se fabrican láseres u otros circuitos fotónicos que propaguen en su seno una alta potencia óptica.

El dopaje con iones activos (por ejemplo de neodimio $\left(\mathrm{Nd}^{+}\right)$) tiene como objetivo brindarle al material propiedades luminiscentes (ó activas), que permiten producir en este material, bajo ciertas condiciones, la emisión estimulada controlada: el principio de funcionamiento de los láseres.

En la Tabla iii, se presenta información general sobre la estructura del $\mathrm{LiNbO}_{3}[\mathrm{LA}]$.

\begin{tabular}{|c|c|}
\hline Banda prohibida (band gap) & 4 ev \\
\hline $\begin{array}{c}\text { Simetría de cristal, clase y grupo de } \\
\text { punto }\end{array}$ & trigonal, R3c y $3 \mathrm{~m}$ \\
\hline Constantes de estructura cristalina & $\mathrm{a}=5.2 \AA$ y c $=13.9 \AA$ \\
\hline
\end{tabular}




\begin{tabular}{|c|c|}
\hline Densidad & $4.648 \mathrm{~g} / \mathrm{cm}$ \\
\hline Dureza Moh & 5 \\
\hline Rigidez a la fractura $\left(\mathrm{MPam}^{0.5}\right)$ & c-face: $0.67 x$-face:1.07 y-face: 1.17 \\
\hline Módulo de elasticidad promedio(GPa) & 206 Gpa \\
\hline Índice de refracción (632nm) & $\begin{array}{c}\mathrm{n}_{\mathrm{o}}=2.286\left(\text { dirección } \mathrm{x} \text { e y), } \mathrm{n}_{\mathrm{e}}=2.2024\right. \\
(\text { dirección } \mathrm{z})\end{array}$ \\
\hline Transmisión óptica & $0.4 \mu \mathrm{m}-5.0 \mu \mathrm{m}$ \\
\hline
\end{tabular}

Tabla iii. Propiedades relevantes del $\mathrm{LiNbO}_{3}$

\subsection{Preparación de muestras.}

Para visualizar las modificaciones estructurales generadas por el láser desde el canto del cristal, acoplar las guías de onda y realizar los mapeos Raman, la superficie de los cantos del cristal deben ser sometidos a un proceso de pulido hasta calidad óptica[FY86].

Primeramente, las muestras utilizadas se desbastaron manualmente, en sucesivas etapas, sobre papel abrasivo al agua (comercialmente denominado "lija al agua") de tamaño de grano 100, 250, 300, 600, 1000 y 1200. Luego se utilizaron, también en sucesivas etapas, pastas de diamante BUEHLER ${ }^{\circledR}$ de $15,6,3,1$ y hasta $1 / 4 \mu \mathrm{m}$ de diámetro medio de grano (Figura 22(b)) colocadas sobre un conjunto de hojas de celulosa $(210 \times 297 \mathrm{~mm})$ adherido mediante cinta adhesiva de papel a una superficie plana de vidrio.

Para llevar a cabo la preparación de una muestra, primeramente se adhirió la misma a un émbolo de acero inoxidable (Figura 22 (c)) en la disposición que se indica en la Figura 22(a), utilizando cera comercial. Los componentes auxiliares mostrados en esta figura también se adhirieron al émbolo de la misma forma. Para tal fin, se elevó la temperatura del conjunto hasta aproximadamente $90^{\circ} \mathrm{C}$ y luego se dejó enfriar el sistema hasta temperatura ambiente. Los topes de acero esquematizados en la Figura 22(a), cumplen la función de mantener la muestra perpendicular a la superficie del émbolo, mientras que los vidrios se adicionan para evitar que los aristas del cristal tomen forma semi-circular por vibraciones del émbolo durante el pulido. A su vez, el émbolo posee una camisa que se puede deslizar en el émbolo como se ve en la Figura 22(c). Este componente, cumple la función de estabilizar el conjunto émbolo-muestra durante su deslizamiento sobre la superficie abrasiva ó pulidora. En todas las etapas, el sistema émbolo-muestra se coloca sobre la superficie de pulido o desbaste de forma tal que la muestra quede en contacto con esta superficie. Entonces, se desliza con movimientos circulares dicho conjunto 
hasta finalizar cada etapa. Las sucesivas etapas (desde abrasivos hasta pastas de pulir) se finalizan cuando dejan de observarse, utilizando una lente de $5 x$ de aumento, irregularidades ó rayas en la superficie del cristal. Esto garantiza un pulido apropiado.

Resulta fundamental entre las sucesivas etapas, la remoción de partículas utilizando abundante agua con disolvente de impurezas. Esto evita que se generen nuevas marcas en la superficie de la muestra que retrasan el tiempo necesario para completar el pulido.

Una vez finalizado el proceso, se realiza nuevamente un calentamiento del conjunto para separar la muestra del émbolo, luego se quita la cera remanente en la muestra utilizando acetona y posteriormente se la limpia con alcohol. En la manipulación de la muestra no deben utilizarse elementos metálicos para evitar la rotura/fisura de la misma por "shock" térmico, principalmente cuando esta se encuentra a temperatura relativamente elevada.

La duración completa del proceso de pulido para dos superficies de los cantos de un cristal de dimensiones convencionales puede demorar aproximadamente $12 \mathrm{hs}$.

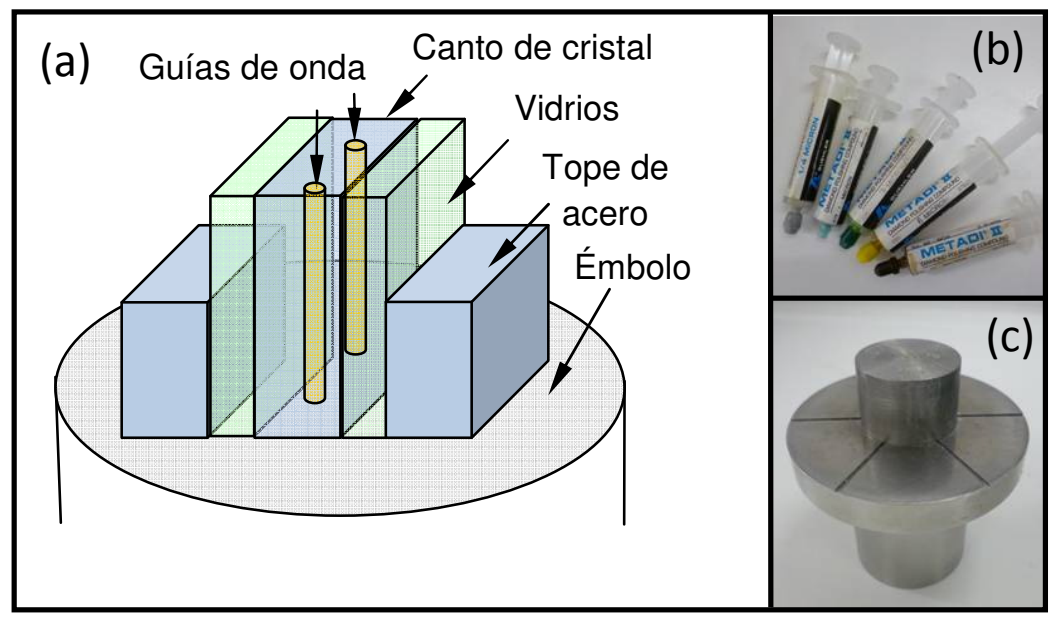

Figura 22. (a) Esquema de disposición de sistema crital-vidrios soporte y topes de acero, (b) pastas de pulir de diamantes BIULHER, (c)imagen de émbolo de pulido

\subsubsection{Preparación de muestra para ensayo bajo presión hidrostática.}

Para realizar el ensayo bajo presión hidrostática, es conveniente que la estructura cristalina esté ordenada para poder seleccionar distintas configuraciones Raman y su superficie pulida para tener una mejor señal Raman. Con lo cual, se requiere que la muestra esté en estado sólido y no en forma granular, como suele utilizarse cuando la orientación de la misma no es relevante, por ejemplo en materiales amorfos.

Debido al reducido tamaño de la cámara de presión (200 $\mu \mathrm{m} \times 50 \mu \mathrm{m}$ aproximadamente), cuando la muestra es sólida debe poseer un espesor menor a $50 \mu \mathrm{m}$, para que, cuando los diamantes presionen la cámara, la muestra no haga contacto directo con estos 
últimos y así evitar rotura o cualquier modificación mecánica fundamentalmente en los diamantes. Este es el principal requerimiento sobre la muestra. Para alcanzarlo, se realizó un desbaste y pulido manual hasta calidad óptica de un trozo de cristal de $\mathrm{Nd}: \mathrm{Mg}: \mathrm{LiNbO}_{3}$ con un tamaño inicial aproximado de $1 \times 1 \times 1 \mathrm{~mm}$. Para el desbaste y pulido manuales, se utilizó en general la metodología explicada en la sub-sección anterior. Las siguientes diferencias se pueden mencionar respecto de la misma:

- se requiere controlar el espesor durante el proceso.

- la muestra no se adhiere directanente al embolo de pulido, sino que se adhiere a una lámina de vidrio de $0.1 \mathrm{~mm}$ de espesor y esta se adhiere al embolo. Esto es necesario porque al finalizar el proceso, permite manipular la muestra con relativa facilidad. A su vez, se adhieren cuatro trozos de vidrio cercanos a la muestra para que la terminación de la misma posea menor desnivel.

- No en necesario obtener una superficie libre de marcas o rayas, por lo tanto no se requieren mayores cuidados durante la abrasión ó el pulido.

- Se requieren los mismos tiempos para las etapas de abrasión y pulido que en el caso anterior. En caso contrario, si se realizan etapas más breves, la abrasión deja modificaciones estructurales/mecánicas en el cristal que distorsionen el espectro Raman del mismo.

Las etapas de desbaste con papel abrasivo se realizaron de igual forma que en el caso anterior. Esta etapa se llevó a cabo hasta que el cristal tuvo aproximadamente $0.1 \mathrm{~mm}$ de espesor, midiéndose con un calibre analógico convencional (Resolución: 0.025 mm).

La segunda etapa consiste en el pulido hasta calidad óptica utilizando pastas de diamante de 15, 6, 3, 1 y 1/4 $\mu \mathrm{m}$. Utilizando la pasta de $15 \mu \mathrm{m}$ se lleva el espesor a 50 $\mu \mathrm{m}$, controlándose con un palpador analógico (Resolución: $0.005 \mathrm{~mm}$ ). Este permite medir diferencias de espesores sin despegar la muestra del émbolo. Utilizando las subsiguientes pastas de pulido y controlando el espesor con el palpador mencionado, se alcanza un espesor menor a $25 \mu \mathrm{m}$. La duración total aproximada de preparación de la muestra fue de 20 hs.

Como resultado de este proceso se obtuvo una muestra de orientación "corte-y", lo que implica que el eje cristalino " $\mathrm{y}_{\mathrm{c}}$ " es perpendicular a su superficie mayor, y con dimensiones aproximadas de $20 \times 500 \times 500 \mu \mathrm{m}$, solidaria a un sustrato de vidrio. 



\section{Modelos elástico-ópticos de elementos finitos}

Durante este trabajo de Tesis se desarrolló un código en entorno MATLAB que calcula deformaciones y modos guiados. El mismo, permitió interpretar las medidas experimentales, tanto de los campos de corrimientos Raman, como así también la distribución de intensidad guiada en guías fabricadas con láser de femtosegundos.

El código generado resuelve, por el Método de Elementos Finitos, el campo de deformaciones generado por una expansión estática arbitraria en el seno de un material con las características elásticas del niobato de litio. Luego, utilizando la relación piezoóptica, obtiene el campo de índice de refracción asociado a dicho campo de deformaciones. Finalmente, calcula los modos de propagación guiados por este campo de índice de refracción, y, los corrimientos Raman esperados de acuerdo a la Teoría de Deformación Potencial.

En este capítulo, se presenta un resumen de los fundamentos del método de elementos finitos aplicado al estudio elástico y de modos guiados. A su vez, se presenta la estructura y finalidad específica de los códigos implementados que se consideran más relevantes. También se incluyen verificaciones que se realizaron durante su generación y las estrategias implementadas para programarlos.

Estos códigos se utilizaron para inducir qué características geométricas tiene la expansión mecánica que ocurre en la interacción laser-material y determinar qué valores de expansión son más adecuados para simular el campo de índice de refracción de las guías estudiadas.

Las ventajas de generar un código en lenguaje MATLAB auto-consistente son: (i) permite adquirir conocimientos sobre la programación de modelos numéricos, conociendo su alcances y limitaciones; (ii) permite implementar fácilmente iteraciones para ajustar los resultados numéricos a medidas experimentales (mapeos Raman ó modos guiados); (ii) al ser un programa/lenguaje ampliamente utilizado en ingeniería y ciencias permitió su difusión para que otros usuarios lo utilicen y/ó modifiquen. 


\subsection{Esquemas de algoritmos}

El objetivo específico de los modelos numéricos generados, es obtener los modos ópticos guiados por efecto piezo-óptico y los corrimientos Raman, producidos por la expansión mecánica arbitraria de una elipse. En la Figura 23, se presenta un esquema resumido de los algoritmos implementados. Los más importantes son: el modelo elástico, que calcula los campos de deformaciones a partir de una expansión mecánica pre-establecida, y, el modelo óptico que obtiene los modos guiados a partir de la distribución de índice de refracción generada por las deformaciones residuales de dicha expansión. Mediante las deformaciones obtenidas con el modelo elástico y aplicando la Teoría de Deformación Potencial Lineal (TPDL) se pueden obtener los corrimientos Raman esperados.

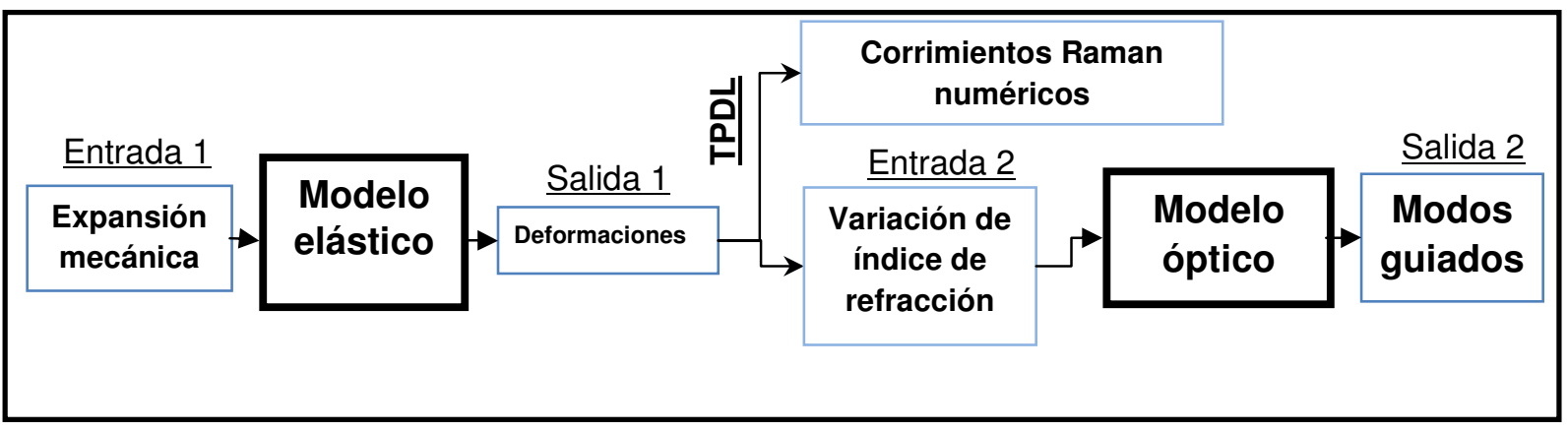

Figura 23. Diagrama de principales algoritmos utilizados y su vinculación: modelo elástico y modelo óptico. TPDL: Teoría Potencial de Deformación Lineal.

En la Figura 24, se presenta un esquema de los algoritmos aclarando las variables de entrada y salida con la nomenclatura utilizada durante el trabajo y las constantes asociadas a los modelos numéricos. 


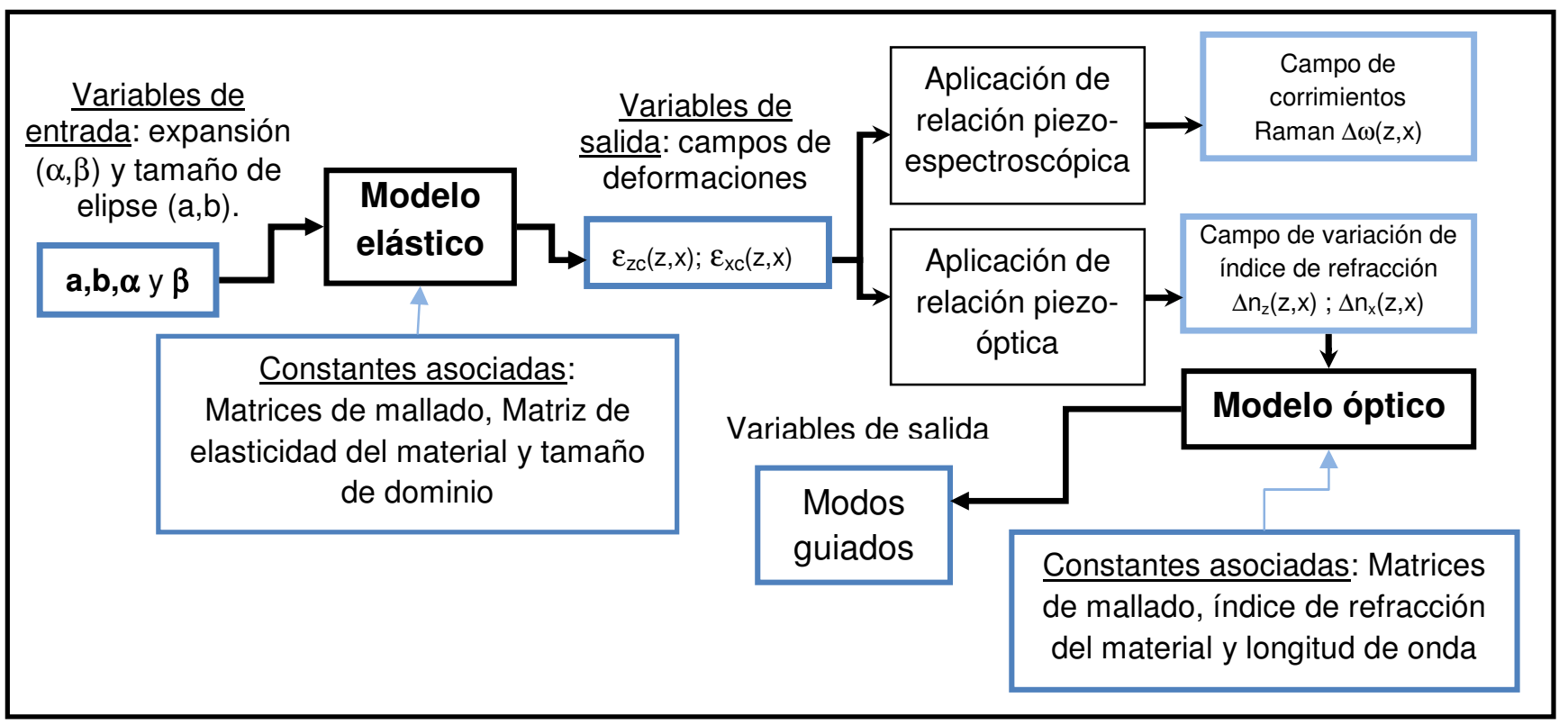

Figura 24. Diagrama de etapas numéricas implementadas en MATLAB: Variables de entrada, de salida y constantes asociadas a cada modelo

\subsection{Método de Elementos Finitos (MEF) para problemas elásticos}

Este método es ampliamente utilizado en ingeniería y ciencias para resolver ecuaciones diferenciales/integrales de forma numérica. En los siguientes párrafos, se resume el origen de la formulación débil para problemas de elasticidad en dos dimensiones. Ésta, es utilizada por programas comerciales como ANSYS, ABAQUS y SOLIDWORKS. Para ampliar sobre esta temática se puede consultar, por ejemplo, las obras de Kwon ó Bathe [KWON97, BA96].

En el planteo de problemas elásticos directos, las incógnitas son los campos de desplazamiento $\left(u_{x}, u_{y}\right.$ y $\left.u_{z}\right)$ de los puntos de un sólido deformable, y a partir de ellos se pueden obtener, mediante un pos-procesado que implica derivaciones, las deformaciones y tensiones correspondientes a cada punto del sólido.

A continuación se describen y presentan las ecuaciones que se vinculan para obtener la formulación que se resuelve en problemas bidimensionales:

(i) Condición de equilibrio: implica que la suma de las fuerzas actuantes sobre un infinitesimal de solido deformable es igual a cero. Por ejemplo, aplicando esta condición en un caso de dos dimensiones, se tiene: 


$$
\begin{gathered}
\sum F_{x}=\left(\sigma_{x}+\partial \sigma_{x}\right) d x d y-\sigma_{x} d x d y+\left(\sigma_{x y}+\frac{\partial \sigma_{x y}}{\partial y}\right) d x d y-\sigma_{x y} d x d y+X_{x} d x d y=0 \\
\sum F_{y}=\left(\sigma_{x y}+\frac{\partial \sigma_{x y}}{\partial x}\right) d x d y-\sigma_{x y} d x d y+\left(\sigma_{y}+\frac{\partial \sigma_{y}}{\partial y}\right) d x d y-\sigma_{y} d x d y+X_{y} d x d y=0
\end{gathered}
$$

Donde $\sigma_{x}$ y $\sigma_{y}$ son las componentes normales y $\sigma_{x y}$ son componentes tangenciales del tensor de tensiones (Figura 25). $X_{x}$ y $X_{y}$ son las denominadas "fuerzas de volumen" (generadas por el campo gravitatorio, un campo magnético, etc.).

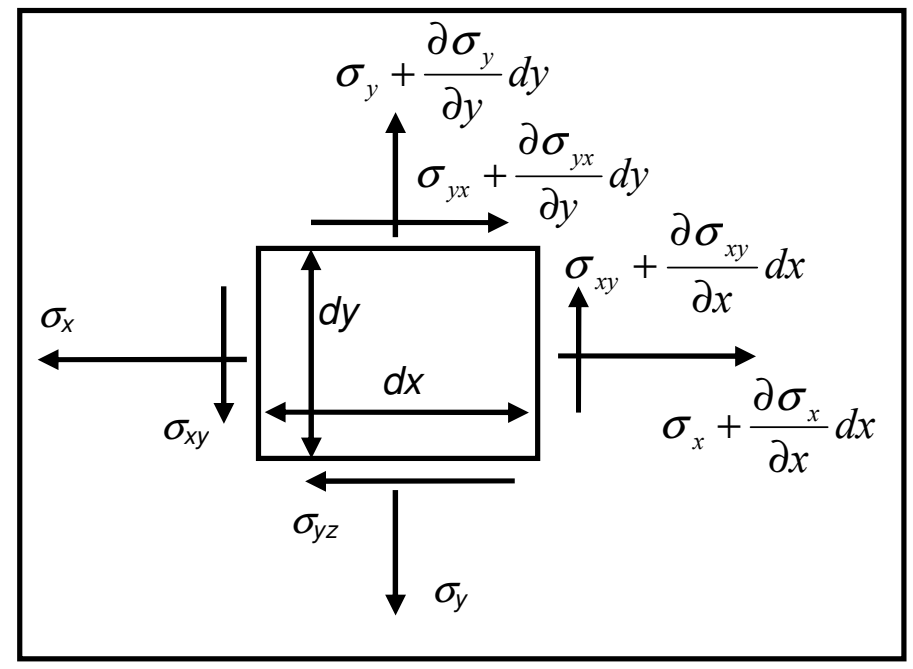

Figura 25. Diferencial de sólido deformable de dos dimensiones

Simplificando estas ecuaciones, se obtiene el siguiente sistema de ecuaciones:

$$
\begin{aligned}
& \frac{\partial \sigma_{x}}{\partial x}+\frac{\partial \sigma_{x y}}{\partial y}+X_{x}=0 \\
& \frac{\partial \sigma_{y}}{\partial y}+\frac{\partial \sigma_{x y}}{\partial y}+X_{y}=0
\end{aligned}
$$

(ii) Relación constitutiva: Como se mencionó en el Capítulo 2, la relación entre las deformaciones y las tensiones en determinado material depende de su composición y estructura cristalina. Aproximando dicho comportamiento a una relación lineal, la relación constitutiva queda definida por la siguiente ecuación matricial: 


$$
\{\sigma\}=\left[C_{r}\right]\{\varepsilon\}
$$

Donde $\left[\mathrm{C}_{\mathrm{r}}\right]$ es la matriz de elasticidad reducida (de $3 \times 3$ elementos).

Habitualmente se asume deformaciones planas $\left(\varepsilon_{x}=0\right)$ ó tensiones planas $\left(\sigma_{x}=0\right)$ para reducir un problema elástico tridimensional a uno bidimensional. En estos casos, la matriz de elasticidad reducida se obtiene a partir de la matriz completa, reemplazando en la Ecuación 22, la condición de deformación o tensión nula que corresponda. Luego se expresan las tensiones en función de las deformaciones.

(iii) Ecuaciones cinemáticas: Son las ecuaciones que relacionan las deformaciones normales y tangenciales con los desplazamientos $\left(u_{x}\right.$ y $\left.u_{y}\right)$. En el caso bidimensional, se tiene la Ecuación 42:

$$
\left\{\begin{array}{c}
\varepsilon_{x} \\
\varepsilon_{y} \\
\varepsilon_{x y}
\end{array}\right\}=\left\{\begin{array}{c}
\frac{\partial u_{x}}{\partial x} \\
\frac{\partial u_{y}}{\partial y} \\
\frac{\partial u_{x}}{\partial y}+\frac{\partial u_{y}}{\partial x}
\end{array}\right\}
$$

Combinando las Ecuaciones 39, 40, 41 y 42, se tiene un sistema de 8 ecuaciones y 8 incógnitas.

(iv) Condiciones de contorno.

Las condiciones de contorno pueden ser esenciales o naturales. Las condiciones esenciales son desplazamientos pre-establecidos (por ejemplo, condiciones de simetría). Las condiciones naturales son fuerzas pre-establecidas y se definen de la siguiente forma:

$\bar{f}_{x}=\sigma_{x} r^{n}{ }_{x}+\tau_{x y} r^{n}{ }_{x}$

$\bar{f}_{y}=\tau_{x y} r^{n}{ }_{x}+\sigma_{y} r^{n}{ }_{y}$

Donde $r^{n}{ }_{x} r_{y}^{n}$ son los cosenos directores normales hacia fuera del dominio y $f_{x}, f_{y}$ son los valores de fuerza por unidad de área.

\subsubsection{Formulación débil de MEF}

Agrupando las ecuaciones anteriores (39 a 44) y aplicando el método denominado "residuos pesados" [KWON97], se puede obtener la formulación débil que se presenta en la Ecuación 45: 


$$
\int_{\Omega}\left[\begin{array}{ccc}
\frac{\partial v_{1}}{\partial x} & 0 & \frac{\partial v_{1}}{\partial x} \\
0 & \frac{\partial v_{2}}{\partial y} & \frac{\partial v_{2}}{\partial y}
\end{array}\right][C]\left\{\begin{array}{c}
\frac{\partial u}{\partial x} \\
\frac{\partial u}{\partial y} \\
\frac{\partial u}{\partial y}+\frac{\partial u}{\partial x}
\end{array}\right\} d \Omega=\int_{\Omega}\left\{\begin{array}{c}
v_{1} X_{x} \\
v_{2} X_{y}
\end{array}\right\} d \Omega+\int_{\Gamma_{N}}\left\{\begin{array}{c}
v_{1} f_{x} \\
v_{2} f_{y}
\end{array}\right\} d \Omega
$$

Donde $\Omega$ es el dominio del sólido deformable y $\Gamma_{N}$ es la trayectoria donde se aplican las condiciones de borde naturales. Los términos a la derecha de la igualdad representan las fuerzas de volumen y las condiciones de borde naturales. $v_{1}, v_{2}$ son las funciones de prueba [KWON97].

A continuación, se describe la aplicación de la Ecuación 45 en dominios discretizados. La obtención de una discretización ó mallado, implica dividir el dominio en regiones denominadas elementos. Los vértices de estas regiones se denominan nodos. En cada elemento, la solución numérica se aproxima a la solución analítica o real, de acuerdo a la característica del elemento.

\section{Estudio elemental}

Discretizando el dominio bidimensional, con elementos triangulares lineales, los desplazamientos en el seno de un elemento pueden ser interpolados utilizando las funciones de forma lineales y los desplazamientos nodales $u_{i}$ y $v_{i}$, como sigue [KWON97]:

$$
\begin{aligned}
& u_{x}(x, y)=\sum_{i=1}^{3} H_{i}(x, y) u_{x i} \\
& u_{y}(x, y)=\sum_{i=1}^{3} H_{i}(x, y) u_{y_{i}}
\end{aligned}
$$

Donde $H_{i}$ son las funciones de forma y $u_{\mathrm{x} i}, u_{\mathrm{yi}}$ son los desplazamientos nodales.

Reemplazando las Ecuaciones 46 y 47 en la ecuación "cinemática" se obtiene la Ecuación 48.

$$
\left\{\begin{array}{c}
\frac{\partial u_{x}}{\partial x} \\
\frac{\partial u_{y}}{\partial y} \\
\frac{\partial u_{x}}{\partial y}+\frac{\partial u_{y}}{\partial x}
\end{array}\right\}=\left[\begin{array}{cccccc}
\frac{\partial H_{1}}{\partial x} & 0 & \frac{\partial H_{2}}{\partial x} & 0 & \frac{\partial H_{3}}{\partial x} & 0 \\
0 & \frac{\partial H_{1}}{\partial y} & 0 & \frac{\partial H_{2}}{\partial y} & 0 & \frac{\partial H_{3}}{\partial y} \\
\frac{\partial H_{1}}{\partial y} & \frac{\partial H_{1}}{\partial x} & \frac{\partial H_{2}}{\partial y} & \frac{\partial H_{2}}{\partial x} & \frac{\partial H_{3}}{\partial y} & \frac{\partial H_{3}}{\partial x}
\end{array}\right]\left\{\begin{array}{l}
u_{x 1} \\
u_{y_{1}} \\
u_{x 2} \\
u_{t 2} \\
u_{x 3} \\
u_{y_{3}}
\end{array}\right\}
$$


La expresión anterior se puede abreviar de la siguiente forma:

$$
\{\varepsilon\}=[B]\left\{u_{i}\right\}
$$

Donde $[B]$ se denomina usualmente matriz cinemática. La misma, multiplicada a los desplazamientos nodales de un elemento, permite obtener un vector con las deformaciones. $u_{i}$ se denomina a los grados de libertad ó desplazamiento de los nodos.

Reemplazando la Ecuación 48 en la Ecuación 45 y aplicando el método de Galerkin $\left(v_{1}=H_{i}\right.$ y $\left.v_{2}=H_{i}\right)$, se obtiene la denominada matriz de rigidez elemental:

$$
\left[K^{e}\right]=\int_{\Omega^{e}}[B]^{T}\left[C_{r}\right][B] d \Omega=[B]^{T}\left[C_{r}\right][B] A_{e}
$$

Donde $A_{\mathrm{e}}$ es el área del elemento.

\subsubsection{Ensamble de matrices elementales}

Una vez obtenidas las matrices de rigidez elementales de todos los elementos, estas son ensambladas. Para tal fin, se suman los elementos de estas matrices elementales, de forma tal, que coincidan sus grados de libertad (desplazamientos nodales $u_{x}$ y $u_{y}$ ). Como consecuencia, se obtiene una matriz de rigidez global de $\mathrm{m} \times \mathrm{m}$. Donde $\mathrm{m}$ es la cantidad de grados de libertad del sistema. Finalmente, se obtiene un sistema de $\boldsymbol{m}$ ecuaciones lineales de la forma:

$$
[K]\left\{u_{i}\right\}=\{f\}
$$

Donde $[K]$ es la matriz de rigidez global, $\left\{u_{i}\right\}$ es el vector de desplazamientos nodales y $\{f\}$ es el vector que contiene las fuerzas externas (condiciones de borde naturales) y las fuerzas de volumen. De esta expresión se puede obtener, por cualquier método de resolución de ecuaciones lineales, el vector $\left\{u_{i}\right\}$. Este representa una solución aproximada al problema elástico planteado.

\subsubsection{Deformaciones planas: aproximación ortotrópica para niobato de litio.}

En la Figura 26(a) se presenta un esquema de la modificación del material generada por el procesamiento láser utilizado en este trabajo. En esta figura, los elipsoides superpuestos representan los volúmenes focales en donde se modifica estructuralmente el material por dicho procesamiento. 
Analizando el origen de la carga aplicada por las sucesivas expansiones solapadas en línea recta, sin tener en cuenta la anisotropía del material ni la característica dinámica de la expansión, se consideró que se correspondía a un estado de deformaciones planas (donde las deformaciones distintas de cero están contenidas en el plano $z_{\mathrm{c}}-\mathrm{x}_{\mathrm{c}}$, mientras que $\varepsilon_{y y}, \varepsilon_{y x}$ y $\varepsilon_{y z}$ son iguales a cero). En la Figura $26(b)$, se esquematizan las sucesivas expansiones solapadas. En esta figura, el círculo rojo representa el volumen focal correspondiente al plano estudiado. Idealmente, las deformaciones normales a este plano producidas por expansiones que están antes del mismo se anulan con los efectos de las que están después de este plano, a medida que evoluciona el procesamiento. Con lo cual, las deformaciones quedaría contenidas en el plano mencionado.

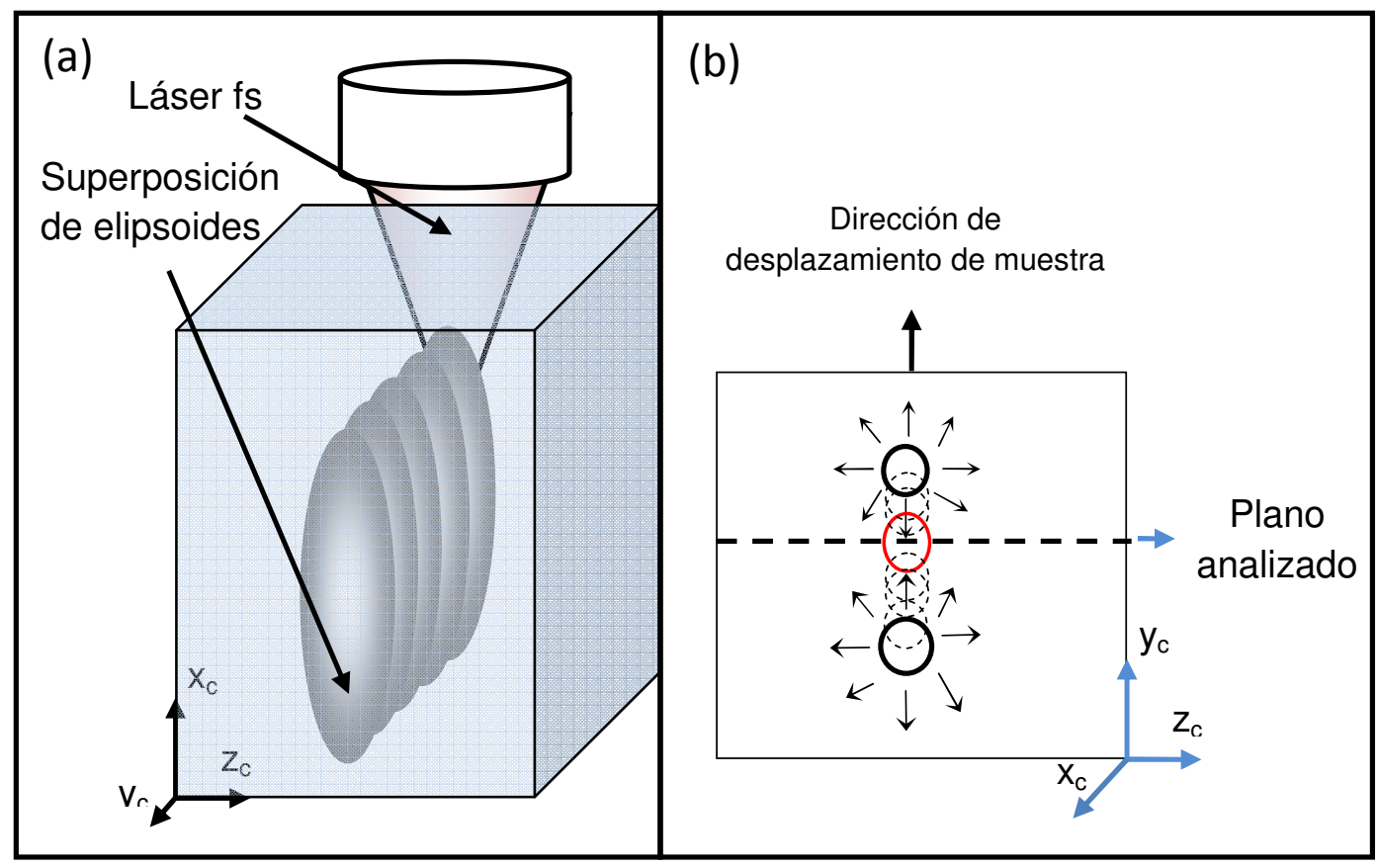

Figura 26. (a)Esquema tridimensional de superposición de volúmenes focales, (b) vista hacia plano $\mathrm{y}_{\mathrm{c}}, \mathrm{z}_{\mathrm{c}}$.

Sin embargo, no todos los materiales anisotrópicos admiten el estado de deformaciones planas. Por ejemplo, siendo estrictos, los materiales trigonales como el niobato de litio no admiten este tipo de simplificación. La razón es que la matriz de elasticidad tiene elementos que vinculan ó "acoplan" las deformaciones contenidas en el plano estudiado con aquellas que no están contenidas en este plano: $\varepsilon_{y c}, \varepsilon_{y x c}$ y $\varepsilon_{y z c}$ en este caso.

Con el fin de aclarar este aspecto se presenta a continuación la matriz de elasticidad [ $C_{\mathrm{ij}}$ ] del niobato de litio. 


$$
\left[\begin{array}{c}
\sigma_{x x} \\
\sigma_{y y} \\
\sigma_{z z} \\
\sigma_{x y} \\
\sigma_{x z} \\
\sigma_{y z}
\end{array}\right]=\left[\begin{array}{cccccc}
C_{11} & C_{12} & C_{13} & C_{14} & 0 & 0 \\
: & C_{11} & C_{13} & -C_{14} & 0 & 0 \\
: & : & C_{33} & 0 & 0 & 0 \\
: & : & 0 & C_{44} & 0 & 0 \\
0 & 0 & 0 & 0 & C_{55} & C_{56} \\
0 & 0 & 0 & 0 & : & C_{66}
\end{array}\right] \times\left[\begin{array}{c}
\varepsilon_{x x} \\
\varepsilon_{y y} \\
\varepsilon_{z z} \\
\varepsilon_{x y} \\
\varepsilon_{x z} \\
\varepsilon_{y z}
\end{array}\right] .
$$

Donde $\mathrm{C}_{11}=200 \mathrm{Gpa}, \mathrm{C}_{22}=200 \mathrm{Gpa}, \mathrm{C}_{33}=235 \mathrm{Gpa}, \mathrm{C}_{12}=53.3 \mathrm{Gpa}, \mathrm{C}_{13}=67.7 \mathrm{Gpa}, \mathrm{C}_{14}=8.7$ Gpa, $C_{44}=53.5 \mathrm{Gpa}, \mathrm{C}_{55}=53.5 \mathrm{Gpa}, \mathrm{C}_{66}=53.5 \mathrm{Gpa}, \mathrm{C}_{56}=9.7 \mathrm{Gpa}$

Para que esta matriz de elasticidad admita deformaciones planas respecto al plano $z_{c} x_{c}$, $\mathrm{C}_{14}$ y $\mathrm{C}_{56}$ tienen que valer cero. Estos son los elementos de la matriz [ $\left.C_{\mathrm{ij}}\right]$ que generan deformaciones por fuera del plano. Porque asocian una tensión contenida en el plano, a deformaciones que, en general, no están contenidas en él. En caso de que $C_{14}$ y $C_{56}$ sean asumidos iguales a cero, la matriz resultante se denominará "aproximación ortotrópica" de la matriz de elasticidad. Esta denominación se asigna porque igualando a cero estos elementos, esta matriz queda equivalente a la de un material ortotrópico. Y en los mismos, es posible (siempre que la carga aplicada no genere desplazamientos salientes del plano) reducir un problema tridimensional a uno bidimensional de deformaciones planas. Para esto, es requerido que el eje normal al plano estudiado coincida con un eje propio del material [T196].

En un primer análisis de la matriz en la Ecuación 52 se observa que los valores de $\mathrm{C}_{14} \mathrm{y}$ $\mathrm{C}_{56}$ son menores a los valores centrales $\left(\mathrm{C}_{11}\right.$ por ejemplo) en más de un orden de magnitud (aproximadamente 20 veces). Esto implica que se podría asumir deformaciones planas respecto al plano $z_{c} x_{c}$, sin cometer errores significativos en el resultado de deformaciones planas. De todas formas, esto se verificó utilizando el programa comercial de elementos finitos ABAQUS [ABM]. Utilizando este programa se resolvieron dos modelos análogos: uno tridimensional, utilizando la matriz completa de elasticidad del niobato de litio y otro bidimensional donde se utilizó la aproximación ortotrópica de este material. Los elementos del modelo tridimensional fueron tetragonales, y los del modelo bidimensional fueron triangulares. En ambos, se aplica una presión interna de igual valor. En la Figura 27 se muestran los esquemas de estos modelos. Las dos elipses tienen la misma geometría. 


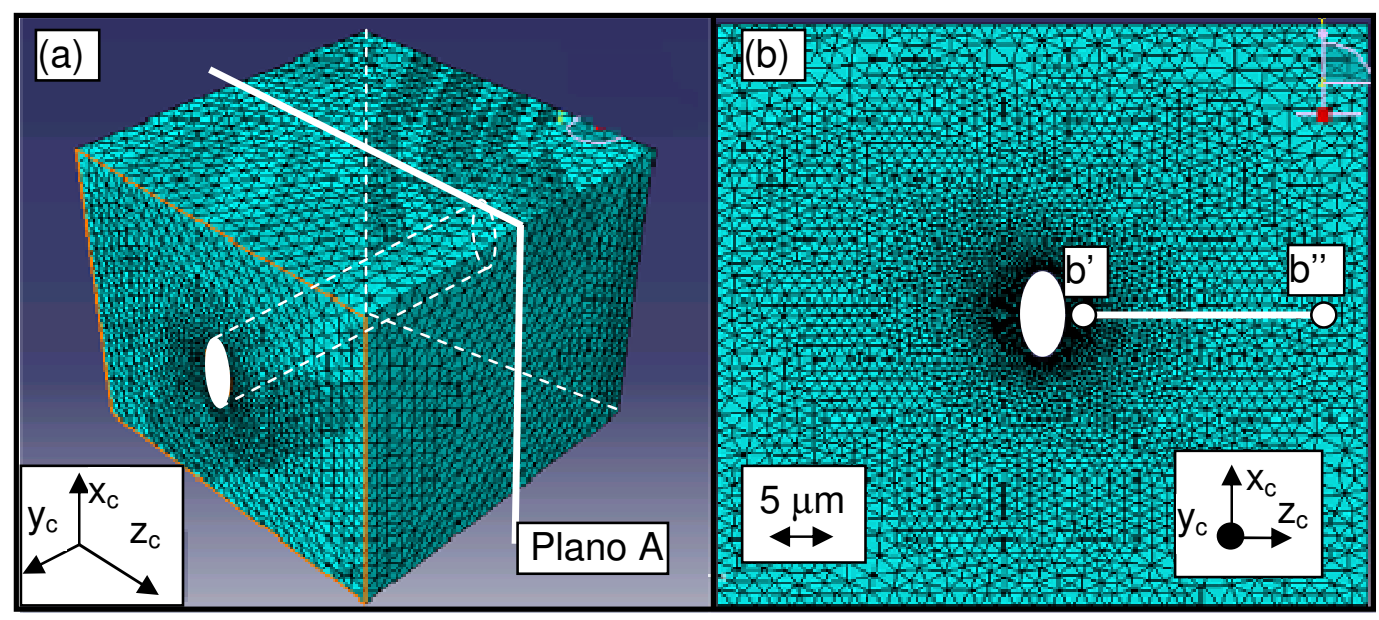

Figura 27. (a) Mallado tridimensional, (b) mallado bidimensional.

Para comparar ambos resultados, se grafican las deformaciones $\varepsilon_{\mathrm{zc}}$ y $\varepsilon_{\mathrm{xc}}$ en la trayectoria b'b" de ambos modelos (Figura 28). Del modelo tridimensional, se utiliza un plano ubicado en el centro del sólido en la dirección $y_{c}$ (Plano A, Figura 27(a)). Se observa que coinciden los valores de deformaciones. Este resultado permite verificar, asumiendo régimen estático y elástico, que la aproximación de deformaciones planas en el plano $\mathrm{z}_{\mathrm{c}} \mathrm{x}_{\mathrm{c}}$ para el niobato de litio no genera diferencias considerables respecto al modelo tridimensional. Por consiguiente, esta aproximación se utilizará en los siguientes apartados para obtener la matriz de rigidez reducida.

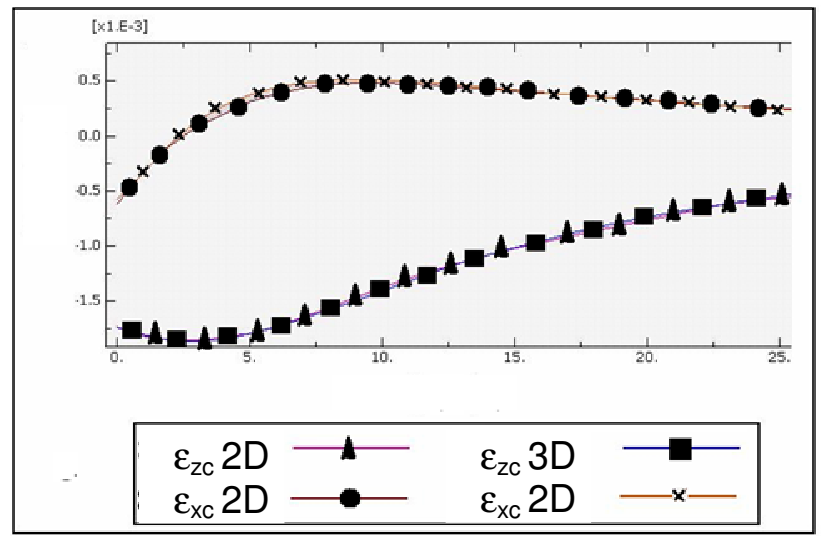

Figura 28. Deformaciones para el modelo bidimensional y para el modelo tridimensional sobre la trayectoria b'b".

\section{Rotación de matriz completa de elasticidad y obtención de matriz reducida.}

Para obtener la matriz reducida de $\mathrm{LiNbO}_{3}$ correspondiente a deformaciones planas en el plano $z_{c} x_{c}$ se generaron y utilizaron los siguientes códigos, siguiendo las indicaciones de T.C.T. Ting [T/96]: 
Partiendo de la matriz de rigidez ortotrópica del niobato de litio, para girarla $90^{\circ}$ alrededor del eje $\mathbf{z}_{\mathrm{c}}$ y otros $90^{\circ}$ alrededor del eje $\boldsymbol{y}_{c}$, se generó el código rot.m (Anexo B).

Para reducir la matriz completa de elasticidad, a una matriz de 2x2 para utilizarla en la aproximación de deformaciones planas, se generó el código "matrizred.m” (Anexo B).

\subsubsection{Parámetros y condición de simetría}

El problema numérico planteado consiste en calcular el campo de deformaciones generado por una expansión estática arbitraria en el seno de un material ortotrópico. A pesar de que no se conoce con exactitud la geometría inicial ni las características geométricas de la expansión (Ver Capítulo 2), teniendo en cuenta los trabajos previos [BUR07], se adopta para el cálculo numérico, una geometría inicial elíptica de radio horizontal a y radio vertical $b$.

A su vez, el fenómeno de expansión mecánica estudiado se asume simétrico. Entonces, se utilizaron dos ejes de simetría. Uno mostrado con el segmento $x_{1} x_{2}$ y otro con el segmento $z_{1} z_{2}$ (Figura 29). Por lo tanto, aplicando las condiciones de contorno adecuadas, el problema se reduce a resolver una expansión mecánica arbitraria en el dominio presentado en la Figura 29.

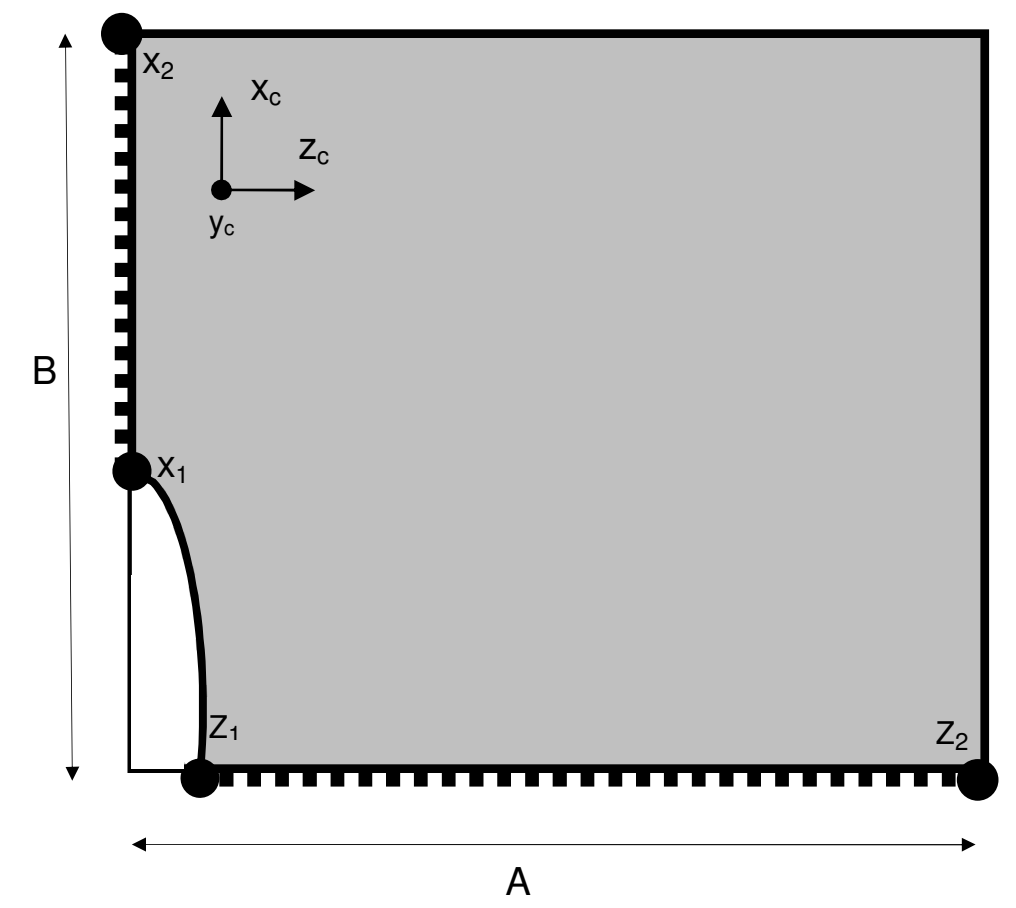

Figura 29. Esquema de cuarto de dominio. 
La condición de contorno pre-establecida para los nodos del borde $\mathbf{z}_{1} \mathbf{z}_{2}$ es que los desplazamientos verticales sean iguales a cero, mientras que el desplazamiento horizontal de aquellos nodos ubicados sobre el margen $\mathbf{x}_{1} \mathbf{x}_{2}$, se establece igual a cero (Figura 29). De esta forma, cualquier expansión que se aplique al cuarto de elipse mostrado, genera un campo de deformaciones simétrico respecto de los ejes propuestos. La reducción del dominio ayuda a disminuir los requerimientos computacionales y facilita la asignación de condiciones iniciales.

Para caracterizar la expansión se utilizaron los parámetros $\alpha$ y $\beta$. Estos se presentan en las Ecuaciones 53 y 54 . Los mismos, en caso de ser iguales entre sí, definen una expansión proporcional (utilizada en trabajos previos [BUR07]). Para este trabajo, en general, no se asume dicha condición de igualdad.

$$
\begin{aligned}
\Delta x_{c i} & =\beta \times x_{c i} \\
\Delta z_{c i} & =\alpha \times z_{c i}
\end{aligned}
$$

Donde $\Delta z_{\mathrm{c}}, \Delta x_{c i}$ son los desplazamientos del nodo i de la elipse en la dirección $z_{c}$ y $x_{c}$, respectivamente. $z_{\mathrm{ci}} \mathrm{y} \mathrm{x}_{\mathrm{ci}}$ la posición de los nodos.

La geometría de la elipse y los parámetros de expansión se esquematizan en la Figura 30. Se puede deducir de la misma, que cuando $\alpha$ sea mayor que $\beta$, la expansión será predominantemente horizontal, y en caso contrario, la misma será predominantemente vertical.

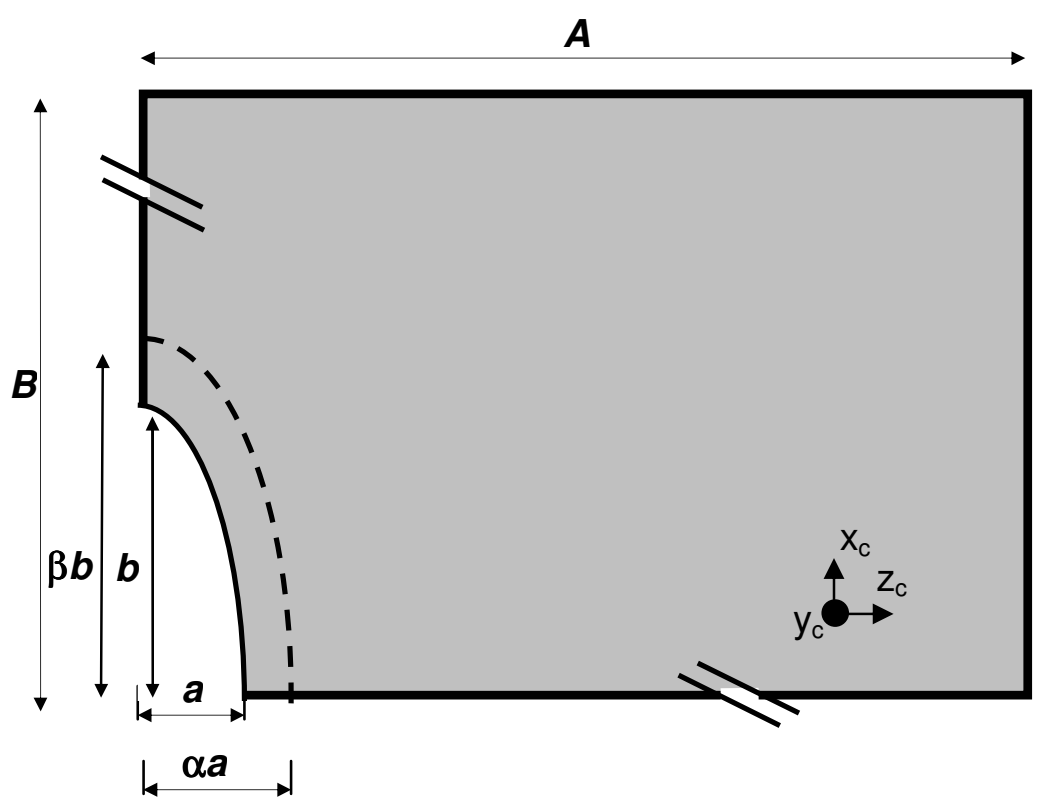

Figura 30. Esquema de parámetros a, b, $\alpha$ y $\beta$. 


\subsubsection{Tamaño de dominio (A y B)}

Para un valor de expansión definido ( $\alpha$ y $\beta$ ), el tamaño de la elipse $(a$ y $b)$ debe ser lo suficientemente menor a las dimensiones $A$ y $B$ para tener una exactitud aceptable en los desplazamientos resultantes [OKA05]. Pero desde el punto de vista del cálculo, aumentar el tamaño de dominio representa, en general, un aumento en el potencial de cálculo necesario y a su vez, aumenta la dificultad de generar un mallado adecuado.

Teniendo en cuenta esto, resulta lógico emplear el mínimo tamaño de dominio que permita obtener una diferencia aceptable entre las deformaciones obtenidas del modelo "reducido" y las del modelo ideal (en el que A y B son mucho mayores que a y b). La magnitud de esta diferencia se denominará tolerancia de deformación $\varepsilon_{\text {tol }}$.

\section{Tolerancia de deformación requerida:}

La tolerancia de deformación requerida depende del método de análisis de guías que se utilice:

- En caso de utilizar medición de mapas micro-Raman, el corrimiento mínimo de pico que se puede detectar es aproximadamente $0.05 \mathrm{~cm}^{-1}$ lo que implica (suponiendo $\mathrm{d} \omega / \mathrm{dp}=2 \mathrm{~cm}^{-1} / \mathrm{GPa}$ ) que la mínima presión detectable ronda los 25 MPa. Utilizando un módulo de elasticidad $E_{c} \approx 200 G p a$ y la conocida expresión $\sigma=\mathrm{E}_{\mathrm{c}} \varepsilon$, esta tensión equivale a una deformación aproximada de $1.3 \times 10^{-4}$ en Niobato de Litio.

- Por otro lado, suponiendo que la variación de índice refracción por debajo de $1 \times 10^{-4}$ no afecta a los modos generados en este tipo de guías [BUR07], mediante la Ecuación 56, (utilizando $\mathrm{p}=0.15$ y $\mathrm{n}=2.20$ ), obtenemos una tolerancia de deformación igual a $1 \times 10^{-4}$ (Esta deformación representa aproximadamente 20 Mpa, en niobato de litio).

$$
\begin{aligned}
\Delta n_{i} & =-\frac{1}{2} \cdot n_{i i}{ }^{3} \sum_{j} p_{i j} \cdot \varepsilon_{j} \\
\varepsilon_{t o l} & =\left|2 \cdot \frac{\Delta n_{i j}}{n_{i i}{ }^{3} \cdot p}\right|
\end{aligned}
$$

Por lo tanto, se asume como tolerable una diferencia entre el dominio ideal y el dominio reducido de $\varepsilon_{\mathrm{tol}}=1 \times 10^{-4}$. 


\section{- Condición A/b}

Cuanto mayor sea la geometría de la elipse, mayor deberá ser el tamaño del dominio para mantener el mismo valor de A/b. A su vez, a mayor magnitud de expansión (considerando $a$ y $b$ fijos) mayores deberán ser $A$ y $B$. Por simplicidad se define $A=B$ y $\alpha=\beta$ en este análisis. Lo relevante, es que se requiere calcular la relación requerida entre el tamaño de dominio, el tamaño de elipse y la magnitud de expansión para que las deformaciones no se aparten, más de lo aceptable, del valor que alcanzan cuando el dominio es infinito o muy grande. Por lo tanto, se definió el parámetro $K^{d}$ :

$K^{\mathrm{d}}=A /\left(b^{*} \alpha\right)$

Se generaron 3 modelos en $A B A Q U S \circledast$ para distintas geometrías de dominio: $A / b=100$, $A / b=25$ y $A / b=15$. En cada uno de estos, se aplicaron 3 expansiones distintas: $\alpha=\beta=0.005, \alpha=\beta=0.01$, y $\alpha=\beta=0.04$. Como resultado se obtuvieron, de los 9 casos mencionados, los campos de deformaciones $\varepsilon_{\mathrm{xc}}, \boldsymbol{\varepsilon}_{\mathrm{zc}}$ y $\varepsilon_{\mathrm{zx}}$. Cada caso es una combinación posible de $\alpha$ y A/b

Para la comparación de los distintos casos se utilizó la componente $\varepsilon_{\mathrm{zc}}$ a lo largo de la trayectoria b'b" (Figura 32). Luego se calculó, para cada uno de los 9 casos, la máxima diferencia entre esta componente de deformación del modelo de dominio reducido y la del máximo dominio $(A / b=100)$, esta diferencia se la denominó tolerancia de deformación $\left(\varepsilon_{t 0}\right)$. En la Figura 31 , se presentan los valores de $K^{\mathrm{d}}$ de cada modelo en función de $\varepsilon_{\text {tol }}$ en escala logarítmica. Se puede ver en esta figura, que se obtiene una relación aproximadamente lineal con cierta dispersión.

El tamaño de dominio óptimo se definió como el mínimo tamaño de dominio cuyo resultado se aleje respecto del dominio mayor $(\mathrm{A} / \mathrm{b}=100)$ una magnitud aproximadamente igual a la tolerancia de deformación requerida $\left(\varepsilon_{\mathrm{tol}}=1 \times 10^{-4}\right)$.

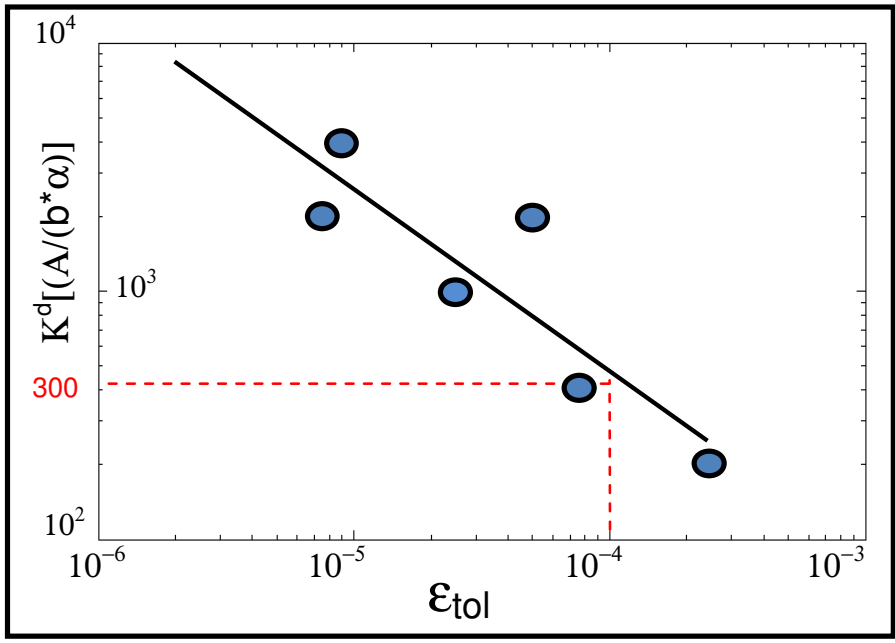

Figura 31. $\mathrm{K}^{\mathrm{d}}$ vs $\varepsilon_{\text {tol }}$ 
Por lo tanto, utilizando la recta presentada en la Figura 31, para una tolerancia $\varepsilon_{\mathrm{tol}}=1 \times 10^{-4}$ se obtiene $\mathrm{K}^{\mathrm{d}}=300$. Considerando, a su vez, una expansión máxima del $5 \%(\alpha=0.05)$ y reemplazando en la Ecuación 19, obtenemos un valor $\mathrm{A} / \mathrm{b}$ mínimo requerido igual a 15 aproximadamente. Esta condición límite, ha sido respetada en todos los modelos numéricos utilizados en el actual trabajo de tesis.

En la Figura 32, se presentan las deformaciones $\varepsilon_{\mathrm{zc}}$ a lo largo de la trayectoria horizontal b'b" (Figura 33) para las diferentes expansiones aplicadas en el modelo donde $A / b=100$.

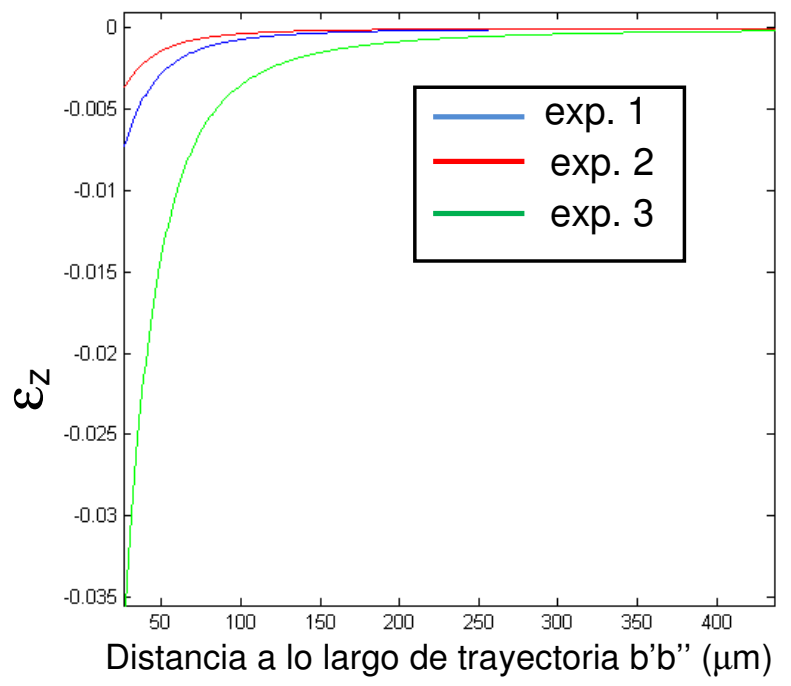

Figura 32. Deformaciones $\varepsilon_{z}$ para distintas expansiones.

En la Figura 33 se muestra el mallado implementado en ABAQUS®.

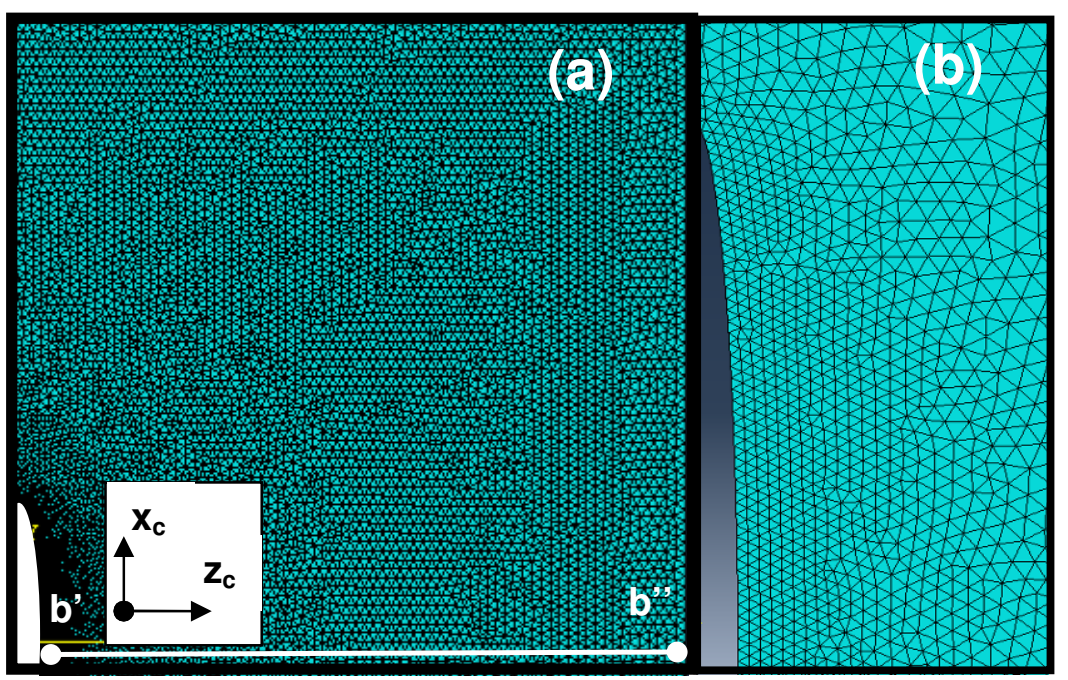

Figura 33. Mallado de elementos triangulares generado en ABAQUS $\AA_{\text {, (a) }}$ discretización general del dominio, (b) detalle en el límite de la elipse. 


\subsubsection{Modelos utilizados y generados en entorno MATLAB}

Es requerido, en el curso de este trabajo, evaluar de forma iterativa el resultado del modelo numérico elástico para distintos valores de parámetros $a, b, \alpha$ y $\beta$ con el objetivo de ajustarlo a las medidas experimentales ó compararlo con las mismas.

Para tal fin, se generaron, en entorno MATLAB, dos modelos cualitativamente diferentes para resolver el problema elástico planteado: uno se utilizó para el análisis unidimensional y otro para el bidimensional. La principal diferencia entre ellos es el tipo de elemento utilizado para la generación del mallado: en el análisis unidimensional se utilizaron elementos cuadrados de 9 nodos y en el análisis bidimensional se utilizaron elementos triangulares de 3 nodos. En la Figura 34, se muestran los elementos mencionados. Para cada tipo de mallado, se necesitaron distintas sub-funciones de discretización como así también, distintas rutinas para aplicar el Método de Elementos Finitos y los correspondientes pos-procesados (obtención de deformaciones a partir de los desplazamientos). Esto se debe a que el algoritmo de integración numérica depende del tipo de elemento utilizado. Estas funciones se presentan en el anexo B.

Entonces, se pueden agrupar dos conjuntos de códigos centrales para resolución y posprocesado: un conjunto asociado al código Codigo1.m y otro asociado al código Codigo2.m. El primero, es utilizado para el análisis unidimensional (elementos cuadriláteros de 9 nodos) y el segundo es utilizado para el análisis bidimensional (elementos triangulares de 3 nodos).

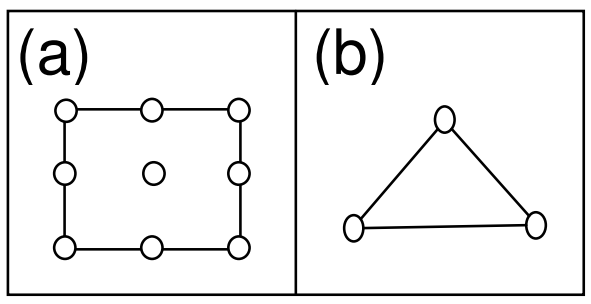

Figura 34. (a) Elemento cuadrado de nueve nodos, (b) elemento triangular de tres nodos.

Como ya se mencionó, el mallado representa la discretización del dominio en el cuál se calculan los desplazamientos desconocidos, en función de cargas o desplazamientos pre-establecidos. Los elementos (estos últimos son las sub-regiones en las que se divide el dominio), deben ser lo más equilátero posible para evitar problemas numéricos en la resolución del sistema matricial [KWON97]. Esta característica, resulta fácil de lograr en dominios de geometría simple, como por ejemplo, cuadriláteros. En cambio, cuando se requiere discretizar ó mallar un dominio con geometrías más complejas, el proceso no resulta trivial. Esta es la dificultad principal de generar un mallado apropiado para el 
dominio estudiado, el cual contiene un cuarto de elipse inserto en un rectángulo. Se puede decir que, en general, ésta es una de las desventajas más relevantes de programar el Método de Elementos Finitos convencional.

- Mallado A: elementos cuadriláteros de 9 nodos

El mallador de generación propia mesh.m tiene la ventaja de que realiza un proceso lineal (sin iteraciones) y como consecuencia a mayor velocidad, en donde las variables de entrada son los parámetros a y b y las variables de salida son las matrices que definen el mallado gcoord y nodes ${ }^{2}$. En la Figura 35 , se esquematizan los nodos generados por este mallador y un elemento genérico de nueve nodos en rojo. En el anexo B se presenta el código mesh.m donde se explican los pasos seguidos por el mismo.

La ventaja de los elementos de nueve nodos es que tienen mayor capacidad de ajuste a una solución abrupta sin necesidad de densificar en gran escala los elementos. La desventaja del mallador generado es que no tiene gran capacidad para adaptarse a los cambios de geometría. Esto se debe a que sigue un procedimiento sistemático para las distintas geometrías y ocurre que para ciertas geometrías de elipse, algunos de los elementos se deforman demasiado y generan discontinuidades de la solución como la que se puede ver en la Figura 36, encerrada por una curva. Estas discontinuidades en la solución, pueden ocupar una región más amplia para otras geometrías de la elipse. Las mismas se forman en la zona lindante superior al cuarto de elipse. Por este motivo, no se utiliza este tipo de mallado en el análisis bidimensional.

Para obtener el campo de deformaciones (Figura 36), para distintos valores de los parámetros $a, b, \alpha$ y $\beta$ (geometría de elipse y expansión) a partir de esta discretización(mallado A), se utilizó el código codigo1.m (presentado en el anexo B).

2 gcoord: es una matriz que posee las coordenadas de los nodos y nodes es una matriz que contiene la conectividad de los mismos para cada elemento, es decir qué nodos conforman cada elemento. 


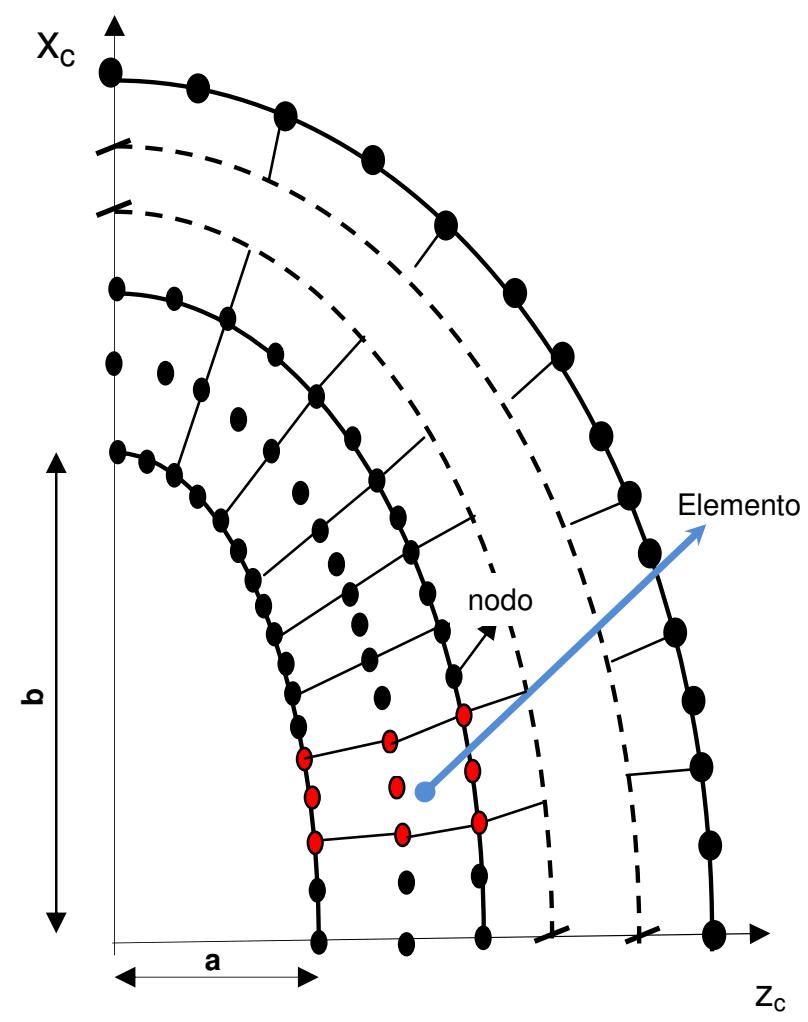

Figura 35. Esquema del mallado A (elementos de nueve nodos).

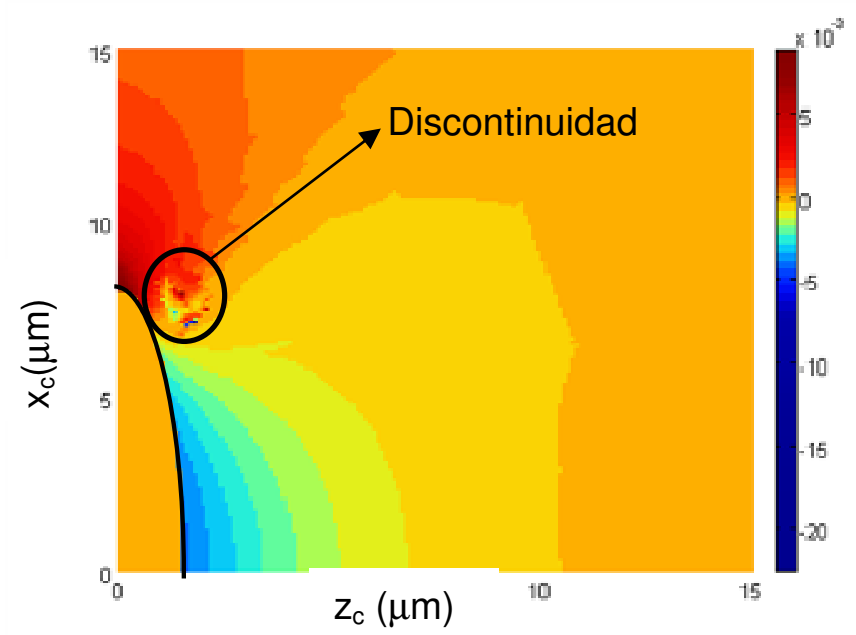

Figura 36. Resultado de deformaciones $\varepsilon_{z c}$ utilizando mallado $A$. 
- Mallado B: elementos triangulares lineales

Para la generación de este mallado, se utilizó el código de Perrson denominado "Distmesh". Este se basa en un proceso iterativo en donde los nodos se van desplazando de acuerdo a una ley de tamaño de elementos establecida por el usuario. Para generar elementos triangulares aproximadamente equiláteros, utiliza una analogía entre las aristas de los elementos y el comportamiento de resortes. Para profundizar sobre este algoritmo generado por Perrson se puede recurrir a su reporte[PER04].

En la Figura 37, se presenta una zona cercana a la elipse del mallado generado para $b / a=2$. En la Figura 38, se presentan las deformaciones $\varepsilon_{\mathrm{zc}}$ obtenidas utilizando el mallado B para una expansión dada por $\alpha=\beta=0.01$.

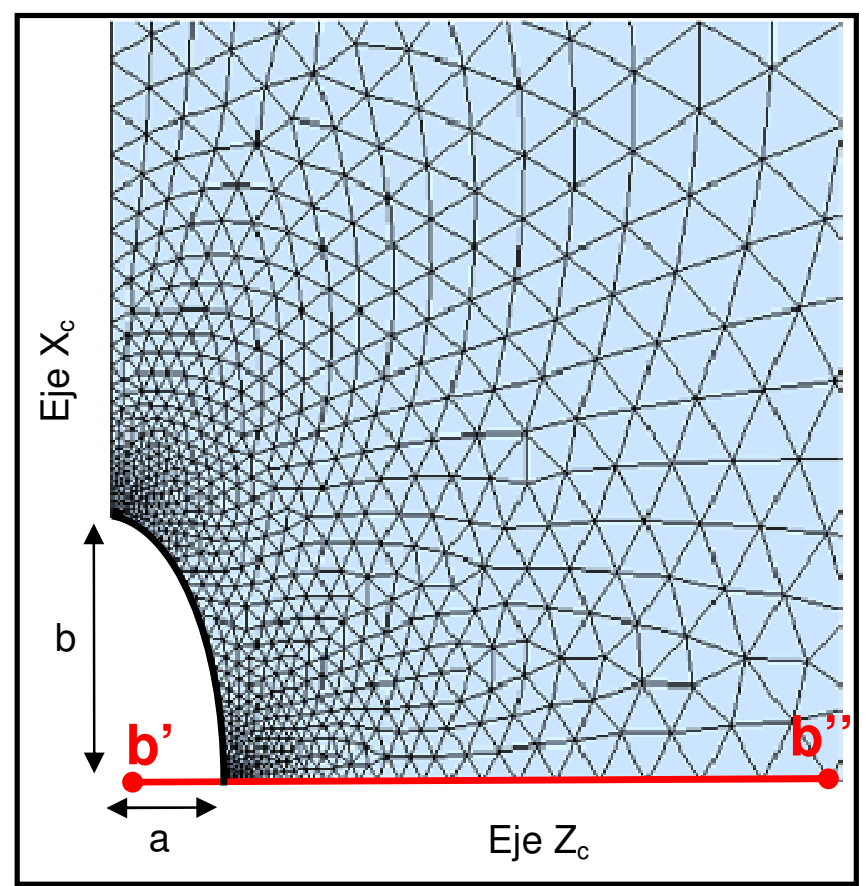

Figura 37. Zona central de mallado B para b/a=2.

Para modificar los parámetros geométricos del modelo ( $a$ y $b$ ) de forma iterativa, este mallador tiene la desventaja de que tarda considerablemente más que el mallador $A$. Además, no es del todo robusto, ya que para determinados parámetros entra en un bucle de cálculo infinito y no finaliza el proceso de mallado. Por lo tanto, para evitar estas complicaciones y poder realizar iteraciones con diferentes valores de geometría de elipse, se siguieron los siguientes pasos:

-Se generaron y almacenaron en arreglos de matrices, diversos mallados para distintos pares de parámetros a y $b$. Para $a$ desde 0.1 a 5 de a intervalos de 0.1 y para $b$ fijo e 
igual a 7. La finalidad es tener pre-calculado y almacenado un mallado ( dado por las matrices gcoord y nodes) correspondiente a cada relación de aspecto b/a.

- Se generó el código selectr.m (Anexo B) que en función de parámetros de entrada $a$ y $b$ calcule la relación de aspecto (b/a), busque las matrices gcoord y nodes pre-calculadas que estén más cercanas a esta relación y luego modifique la posición de los nodos (gcoord) de acuerdo a los valores de $a$ y $b$ deseados.

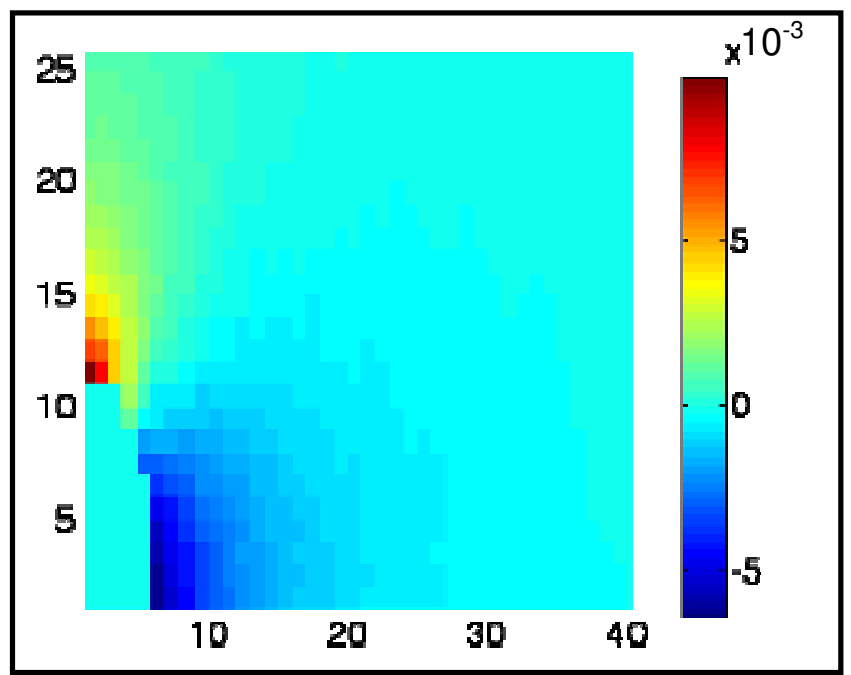

Figura 38. Resultado de deformaciones $\varepsilon_{\mathrm{zc}}$ utilizando mallado $B$.

\subsubsection{Comparación de modelos en MATLAB con programa comercial ABAQUS}

Comparando la Figura 36 y la Figura 38, se puede observar que los dos modelos evaluados tienen soluciones análogas: una zona adyacente de deformación $\varepsilon_{\mathrm{zc}}$ negativa, cuya magnitud tiende a cero conforme aumenta la distancia horizontal a la elipse central. A su vez, $\boldsymbol{\varepsilon}_{z c}$ toma valores positivos en la parte superior de la elipse y su magnitud disminuye, conforme aumenta la distancia a la elipse, hasta valer cero.

Para verificar el correcto desempeño de los códigos generados en MATLAB para ambos malladores (mallado A y mallado B), se realizó una comparación entre la solución obtenida con estos y la obtenida utilizando el programa comercial ABAQUS. Utilizando en los 3 modelos iguales parámetros: una expansión proporcional $\alpha=\beta=0.01$, una geometría de elipse de $a=5$ y $b=10 \mu \mathrm{m}$, un dominio $A / b>15$ y un material isotrópico de módulo de elasticidad $E_{k}=0.2 \mathrm{~N} / \mu \mathrm{m}$ y coeficiente de Poisson: $v^{\mathrm{p}}=0.3$.

Para facilitar su comparación, se presenta, en la Figura 39, la deformación $\varepsilon_{z c}$ a lo largo de la trayectoria $\boldsymbol{b}^{\prime} \boldsymbol{b}$ '” (presentada en la Figura 37 ) para los tres modelos mencionados. Se eligió esta trayectoria porque las guías de onda se reportaron principalmente en esta 
zona en trabajos previos, y, porque se utilizará la misma trayectoria en los ajustes unidimensionales de los corrimientos Raman. Por consiguiente, es importante verificar la convergencia de los diferentes modelos en la misma.

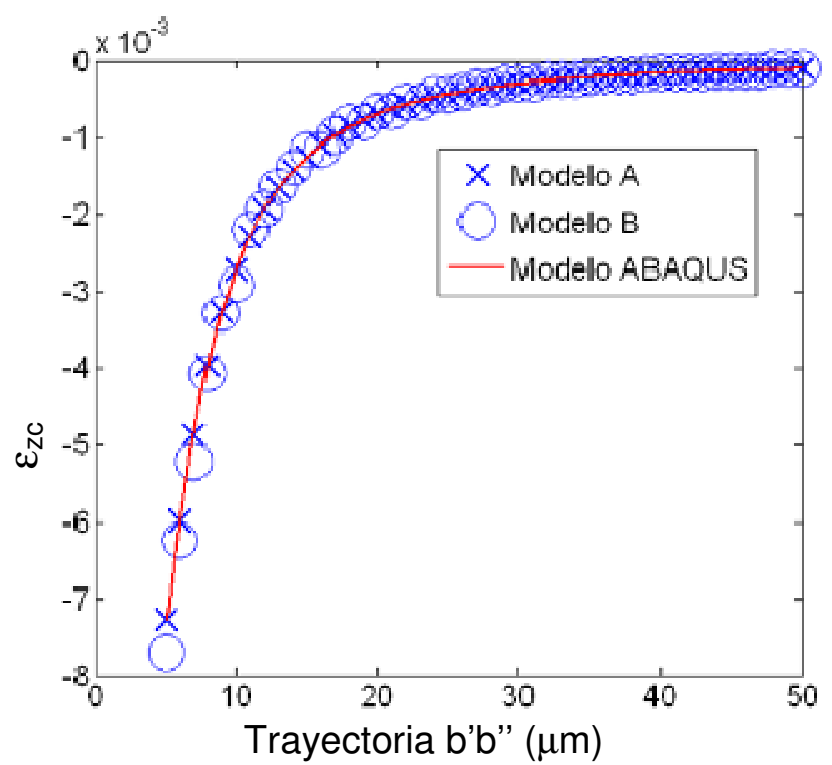

Figura 39. Comparación entre los resultados de los códigos auto-contenidos generados en MATLAB y aquel resultante de utilizar el programa ABAQUS.

Se puede ver en la Figura 39, que los modelos numéricos generados en MATLAB, tanto utilizando el mallado $A$ y el mallado $B$, dan resultados equivalentes al del software comercial. La comparación cuantitativa de los modelos numéricos, se realiza con una sola variable $\left(\varepsilon_{\mathrm{zc}}\right)$ y en una trayectoria lineal para facilitar su interpretación.

En conclusión, ambos códigos generados permiten la resolución de la ecuación de elasticidad para materiales que admitan deformaciones planas (en este trabajo se utilizó la aproximación ortotrópica de $\mathrm{LiNbO}_{3}$ ), en donde, una geometría elíptica se expande en forma arbitraria. Aunque, en el caso del mallador $A$, se observó que genera discontinuidades en la solución en una zona lejana a la trayectoria b'b". Por esta razón, en los análisis unidimensionales se utilizaron mallados de tipo A y su código asociado (Codigo1.m), y, para los estudios bidimensionales se utilizaron mallados de tipo B y su código (Codigo2.m) y subfunciones asociadas (Anexo B).

Cabe mencionar que los códigos implementados para realizar ajustes, iteraciones y posprocesado fueron creados en su mayoría por el autor de esta tesis. El mallador A también fue generado por este, mientras que el mallador B (Distmesh.m) fue generado por Perrson [PER04]. La autoría de los códigos utilizados se detalla en el Anexo B. 


\subsection{Cálculo de variación de índice de refracción}

Para obtener la variación de índice de refracción en el dominio estudiado por efecto de las deformaciones, se utilizó la relación piezo-óptica presentada en las Ecuaciones 58 y 59 mediante el código Campoindtri2.m. Los valores de las constantes piezo-ópticas $\left(p_{\mathrm{ii}}\right)$ utilizadas, se presentan en la Tabla i.

$$
\begin{aligned}
& \Delta n_{x c}=\frac{1}{2} n_{x c}{ }^{3}\left(p_{11} \cdot \varepsilon_{x c}+p_{13} \cdot \varepsilon_{z c}\right) \\
& \Delta n_{z c}=\frac{1}{2} n_{z c}{ }^{3}\left(p_{31} \cdot \varepsilon_{x c}+p_{33} \cdot \varepsilon_{z c}\right)
\end{aligned}
$$

\subsection{Modelo óptico: Cálculo de modos guiados}

Como se mencionó en el Capítulo 2, para calcular los modos de propagación guiados utilizando la aproximación escalar, es necesario resolver la ecuación diferencial de Helmholtz (Ecuación 60). En lugar de resolver esta ecuación directamente, la solución a la misma se puede obtener encontrando la solución al problema variacional, que haga estacionario el funcional presentado en la Ecuación 61. Entonces, resolviendo la Ecuación 62 se pueden obtener los modos de propagación en una distribución de índice arbitraria [OKA05].

$$
\begin{aligned}
& \nabla^{2} \Phi+\left[k^{2} n^{2}\left(z_{c}, x_{c}\right)-\beta^{2}\right] \Phi\left(z_{c}, x_{c}\right)=0 \\
& I[\Phi]=\frac{1}{2} \int_{-\infty-\infty}^{+\infty+\infty} \int\left[\left(\frac{\partial \Phi}{\partial z_{c}}\right)^{2}+\left(\frac{\partial \Phi}{\partial x_{c}}\right)^{2}-\left(k^{2} n^{2}-\beta_{p}\right) \Phi^{2}\right] d z_{c} d x_{c} \\
& \frac{\partial I}{\partial \Phi}=0
\end{aligned}
$$

En la aplicación del Método de Elementos Finitos para resolver la Ecuación 62, al igual que en el problema elástico, es necesario discretizar el dominio en subregiones. Las mismas son triangulares en el presente estudio. Estas regiones, como ya se mencionó, se denominan elementos y sus vértices se denominan nodos. Nodos y elementos conforman el mallado del dominio. En esta subsección los nodos de elementos triangulares, se denominan $i, j$ y $k$. En la Figura 40 (b) se presenta el esquema de un elemento genérico del dominio. 


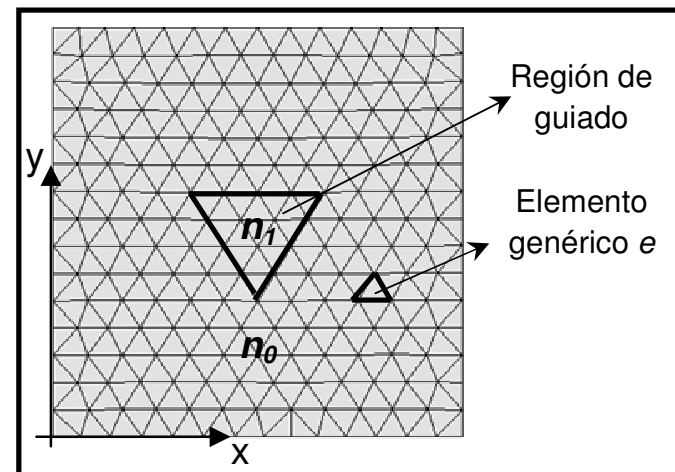

(a)

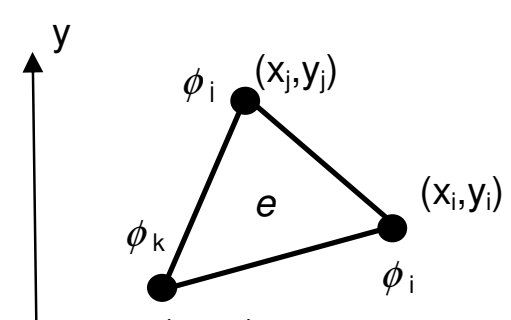

(b)

Figura 40 (a) Mallado de región de guiado y adyacencias en una guía "escalón",(b) esquema de elemento genérico $e$, se denotan las coordenadas de los nodos $y$ valores de campo eléctrico nodales $\phi$.

En un elemento genérico e, el campo eléctrico $\phi$ es aproximado como una función lineal de $x$ e $y$ :

$\phi(x, y)=p_{0}^{e}+p_{1}^{e} x+p_{2}^{e} y$

Donde $p_{k}{ }^{e}$ son parámetros que dependen de la geometría del elemento y de los valores del campo eléctrico en cada nodo del elemento:

$\left|\begin{array}{c}p_{o}{ }^{e} \\ p_{1}{ }^{e} \\ p_{2}{ }^{e}\end{array}\right|=\left(\begin{array}{ccc}1 & x_{i} & y_{i} \\ 1 & x_{j} & y_{j} \\ 1 & x_{k} & y_{k}\end{array}\right)^{-1}\left(\begin{array}{c}\phi_{i} \\ \phi_{j} \\ \phi_{k}\end{array}\right)$

Como el funcional $I$ es una magnitud escalar, resulta práctico definir el funcional elemental $I^{\mathrm{e}}$ para el elemento genérico e y luego realizar una sumatoria. Reemplazando las Ecuaciones 63 y 64 en la Ecuación 61, y operando, se puede obtener la siguiente ecuación:

$$
\begin{aligned}
& I^{e}=\frac{1}{8 S_{e}}\left[\left(y_{j}-y_{k}\right) \phi_{i}+\left(y_{k}-y_{i}\right) \phi_{j}+\left(y_{i}-y_{j}\right) \phi_{k}\right]^{2}+\frac{1}{8 S_{e}}\left[\left(x_{k}-x_{j}\right) \phi_{i}+\left(x_{i}-x_{k}\right) \phi_{j}+\left(x_{i}-x_{i}\right) \phi_{k}\right]^{2} \\
& -\frac{\left(k^{2} n_{e}{ }^{2}-\beta_{p}{ }^{2}\right) S_{e}}{12}\left(\phi_{i}^{2}+\phi_{j}^{2}+\phi_{k}^{2}+\phi_{i} \phi_{j}+\phi_{j} \phi_{k}+\phi_{k} \phi_{i}\right)
\end{aligned}
$$

Donde $x_{i}, y_{\mathrm{i}}, x_{\mathrm{j}}, y_{\mathrm{j}}, x_{\mathrm{k}}$ y $y_{\mathrm{k}}$ son las coordenadas de los nodos del elemento triangular $e ; \phi_{1}, \phi_{\mathrm{j}}$ y $\phi_{\mathrm{k}}$ son valores del campo eléctrico en los nodos del elemento, $S_{\mathrm{e}}$ representa el área del elemento, $n_{\mathrm{e}}$ es el índice de refracción en el elemento $e, k$ es el número de onda de la radiación propagada $(2 \pi / \lambda), \quad \beta_{\mathrm{p}}$ la constante de propagación; estos dos últimos parámetros son constantes para el dominio completo. 
Derivando la Ecuación 65, respecto de $\phi_{i}$ como indica la Ecuación 62, se obtiene la siguiente ecuación:

$$
\begin{aligned}
& \frac{\partial I^{e}}{\partial \phi_{i}}=\left\{\frac{1}{S_{e}}\left[(y i-y k)^{2}+\left(x_{k}-x_{j}\right)^{2}\right]-\frac{2}{3} k^{2}\left[n_{e}^{2}-\frac{\beta^{2}}{k^{2}}\right] S_{e}\right\} \phi_{i}+ \\
& +\left\{\frac{1}{S_{e}}\left[\left(y_{j}-y k\right)\left(y_{k}-y_{i}\right)+\left(x_{k}-x_{j}\right)\left(x_{k}-x_{j}\right)\right]-\frac{1}{3} k^{2}\left[n_{e}{ }^{2}-\frac{\beta^{2}}{k^{2}}\right] S_{e}\right\} \phi_{j}+ \\
& +\left\{\frac{1}{S_{e}}\left[\left(y_{j}-y_{k}\right)\left(y_{i}-y_{j}\right)+\left(x_{k}-x_{j}\right)\left(x_{j}-x_{i}\right)\right]-\frac{1}{3} k^{2}\left[n_{e}{ }^{2}-\frac{\beta^{2}}{k^{2}}\right] S_{e}\right\} \phi_{k}
\end{aligned}
$$

Donde todos los parámetros tienen valores conocidos, a excepción de la constante de propagación $\beta$ y de la magnitud de campo eléctrico en los nodos $\phi_{1}, \phi_{1}$ y $\phi_{k}$.

Derivando de forma análoga, $\mathcal{P}$ respecto de $\phi_{\mathrm{j}}$ y $\phi_{\mathrm{k}}$, se pueden obtener las expresiones de $\partial I^{e} \partial \phi_{\mathrm{j}}$ у $\partial I^{e} \partial \phi_{\mathrm{k}}$.

El funcional del sistema está dado por la suma de los $N$ funcionales elementales $I^{\mathrm{e}}$ :

$$
I^{g}=\frac{1}{2} \sum_{e=1}^{N} I^{e}
$$

Donde $N$ es la cantidad total de elementos.

Por lo tanto, sumando las derivativas $\partial I^{e} \partial \phi_{\mathrm{i}}$ de los $N$ elementos, se obtiene una ecuación lineal con $m+1$ incógnitas igualada a cero ( $m$ es la cantidad de nodos del sistema). Estas incógnitas son: los valores nodales de $\phi$ y el valor de la constante de propagación $\beta_{p}$. Haciendo lo propio para las derivativas $\partial I^{e} \partial \phi_{\mathrm{j}}$ y $\partial I^{e} \partial \phi_{\mathrm{k}}$ y agrupando convenientemente los términos, se puede obtener un sistema de ecuaciones lineales que, con notación matricial, se puede expresar de la siguiente forma:

$$
\left(\left[I^{g}\right]-\beta_{p}\left[J^{g}\right]\right)\{\phi\}=0
$$

Donde $\left.{ }^{[\rho}\right]$ y $\left.{ }^{\mathcal{F}}\right]$ son matrices conocidas que dependen del mallado del dominio, del campo de índice de refracción pre-establecido y de la longitud de onda propagada; $\beta_{p}$ es la constante de propagación desconocida y $\{\phi\}$ es un vector desconocido cuyas componentes son las amplitudes del campo eléctrico en cada nodo.

En la Ecuación 68, se puede ver fácilmente que $\beta_{\mathrm{p}}$ y $\{\phi\}$ representan los autovalores y autovectores de la matriz $[A]$, definida como el producto matricial $\left[{ }^{g}\right]^{-1}\left[I_{g}\right]$. Entonces, la distribución de campo eléctrico, para cada modo guiado, se puede calcular determinando 
los autovectores de la matriz conocida $[A]$ y cada constante de propagación correspondiente, calculando sus autovalores.

De esta forma, este procedimiento permite obtener la distribución de campo eléctrico para una distribución arbitraria de índice de refracción. Para profundizar en esta temática, se puede recurrir, por ejemplo, a la obra de Okamoto [OKA05].

\subsubsection{Cálculo de modos guiados en guía plana}

Para aplicar el procedimiento explicado en la subsección anterior a una guía plana, es decir, para una distribución de índice de refracción unidimensional, sólo es necesario anular la dimensión que corresponda en las Ecuaciones 61 a 68 . Aplicando esta simplificación, se generó el algoritmo Modos1D.m (Anexo B) en MATLAB para calcular la distribución de campo eléctrico en un dominio unidimensional. Esto se realizó para verificar la correcta aplicación del método presentado. Se calcularon los modos guiados en una guía "escalón" determinada por el perfil de índice de refracción presentado en la Figura 41(a). En la Figura 41(b), se presentan los modos obtenidos utilizando el código generado en entorno MATLAB, y en la Figura 41(c) se presentan los modos calculados con el programa comercial R-Soft ${ }^{3}$ para el mismo perfil de índice. En estas figuras, se observa que la distribución de campo eléctrico concuerda entre ambos modelos. La escala de las amplitudes de los modos guiados en ambas figuras es arbitraria.

${ }^{3}$ La herramienta utilizada de este programa se denomina BMP (Método de Propagación de Rayos), permite calcular distribuciones de campo electromagnético y se basa en la técnica numérica conocida como FDTD (http://optics.synopsys.com/rsoft/rsoft-passive-devicebeamprop.html) . 
100 Caracterización de guías de onda ópticas generadas con láser de femtosegundos en Niobato de Litio

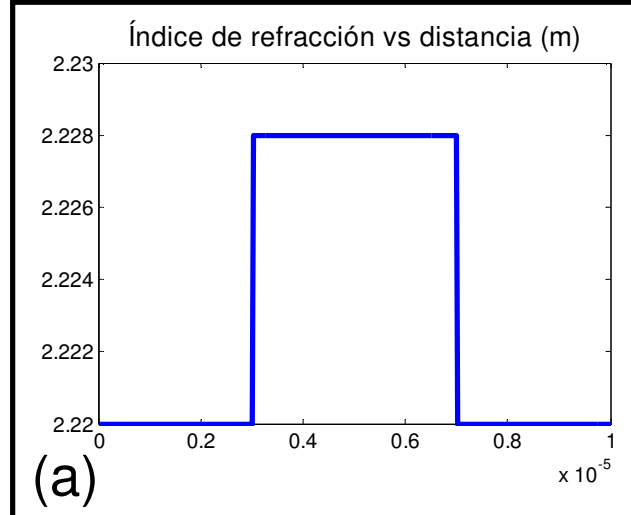

Figura 41. Cálculo de modos guiados en guía de onda plana,(a) perfil de índice utilizado,(b) modos guiados utilizando código MATLAB,(c) modos guiados utilizando RSoft ${ }^{\circledR}$.

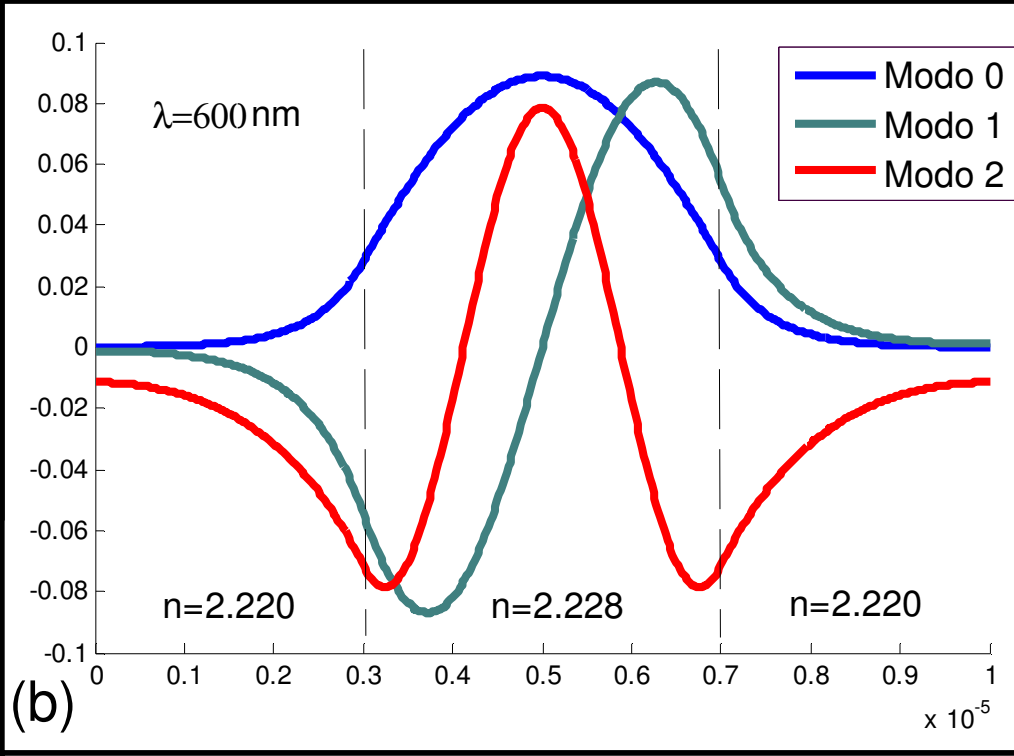

Computed Transverse Mode Profile $\left(m=0, n_{\text {eff }}=2.227201\right)$

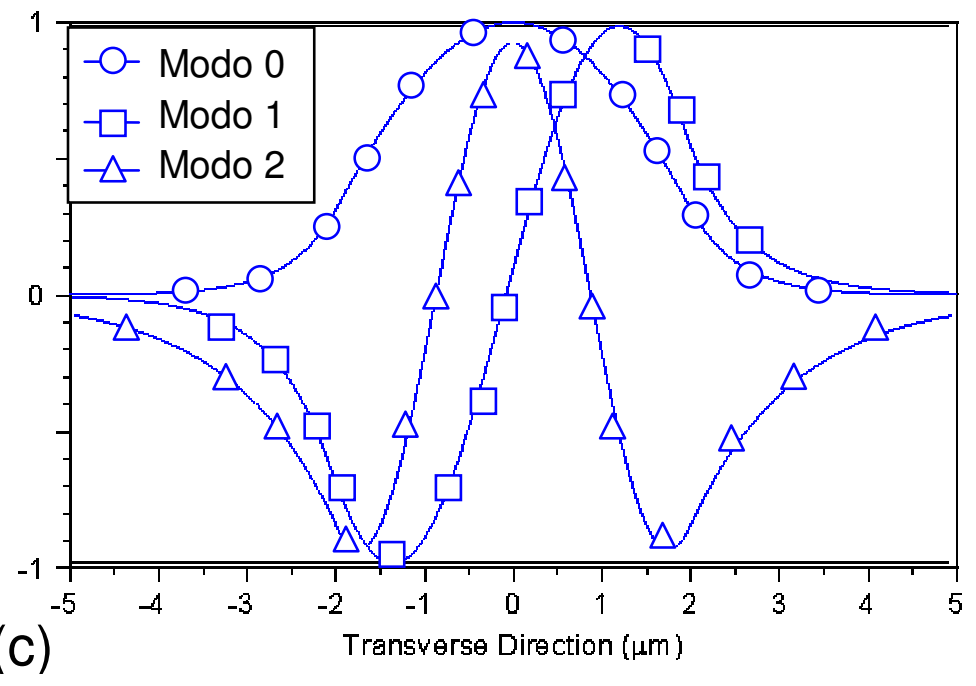

\subsubsection{Modos guiados en campo de índice de refracción generado por una expansión mecánica}

Para calcular la distribución de intensidad guiada por un campo de índice de refracción generado por deformaciones originadas por la expansión de una elipse de radios $a, b$ y con una expansión $\alpha$ y $\beta$, se generó un nuevo mallado. El mismo, se definió con las matrices gcoord2 y nodes2, utilizando el código distmesh2d.m, por intermedio de la instrucción mallador3.m (Anexo B). El dominio generado es rectangular de lados de dimensiones $A$ y 2xA. Los elementos son triangulares lineales y se densifican en las cercanías de la elipse con una ley que depende linealmente de la distancia a los focos que se muestran en la Figura 42(b). Se utilizaron elementos triangulares lineales para facilitar la implementación del método de cálculo. 
Se generó este nuevo mallado para el cálculo de los modos guiados, porque la zona de modificación estructural (representada como una elipse en el modelo elástico) es necesario que forme parte del dominio, debido a que en esta zona el material presenta una disminución importante de índice de refracción.

El mallado se muestra en la Figura 42. Donde $b=9 \mu \mathrm{m}, a=2 \mu \mathrm{m}$ y $A=135 \mu \mathrm{m}$.

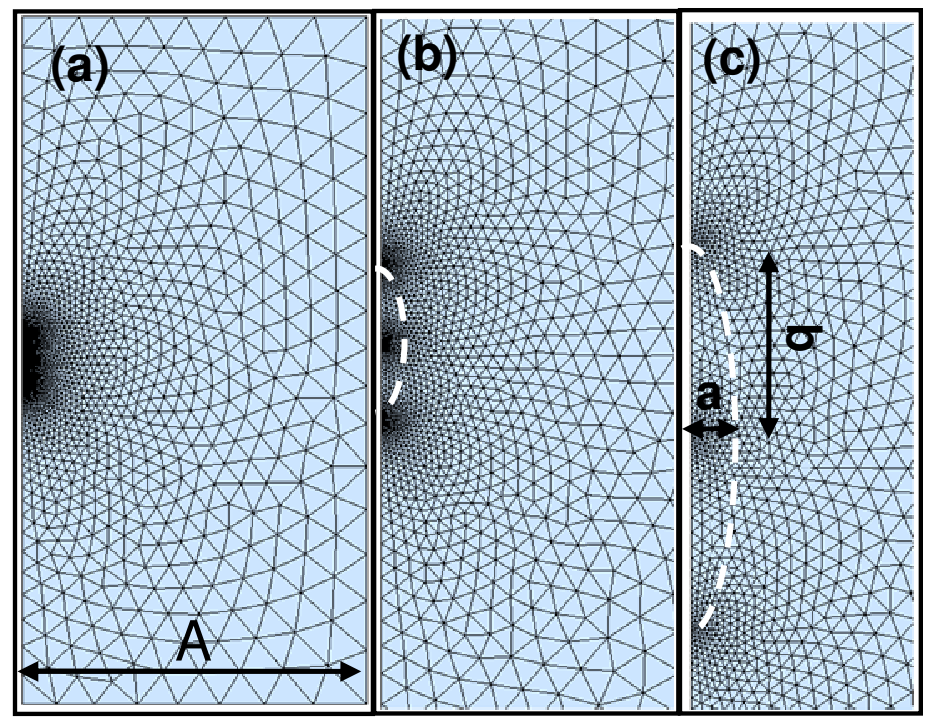

Figura 42. (a) Dominio para cálculo de intensidad guiada, (b) zona central del mallado, (c) Características de la densificación del mallado.

A partir de las deformaciones $\varepsilon_{\mathrm{xc}}$ y $\varepsilon_{\mathrm{zc}}$ calculadas, mediante el código codigo2.m (Sección 4.2.6), para una determinada expansión (Figura 43(a) y (b)), se utilizó el código campoindtri2. $m$ para obtener la distribución del índice de refracción, por intermedio de la relación piezo-óptica (Sección 4.3) para el dominio estudiado. Se asignó una disminución de índice de refracción constante igual a $5 \times 10^{-3}$ a la región delimitada por la elipse. La variación de la componente $x_{c}$ del índice de refracción obtenida se muestra en la Figura 43(c). Luego, utilizando el código codigo3.m, se calculó la distribución de intensidad guiada que se muestra en la Figura 43(d). La misma corresponde a la polarización en dirección $x_{c}$ (vertical) y a una expansión estática de $\alpha=\beta=0.003$ de una elipse de radios $a=2 \mu \mathrm{m}$ y $b=9 \mu \mathrm{m}$. 


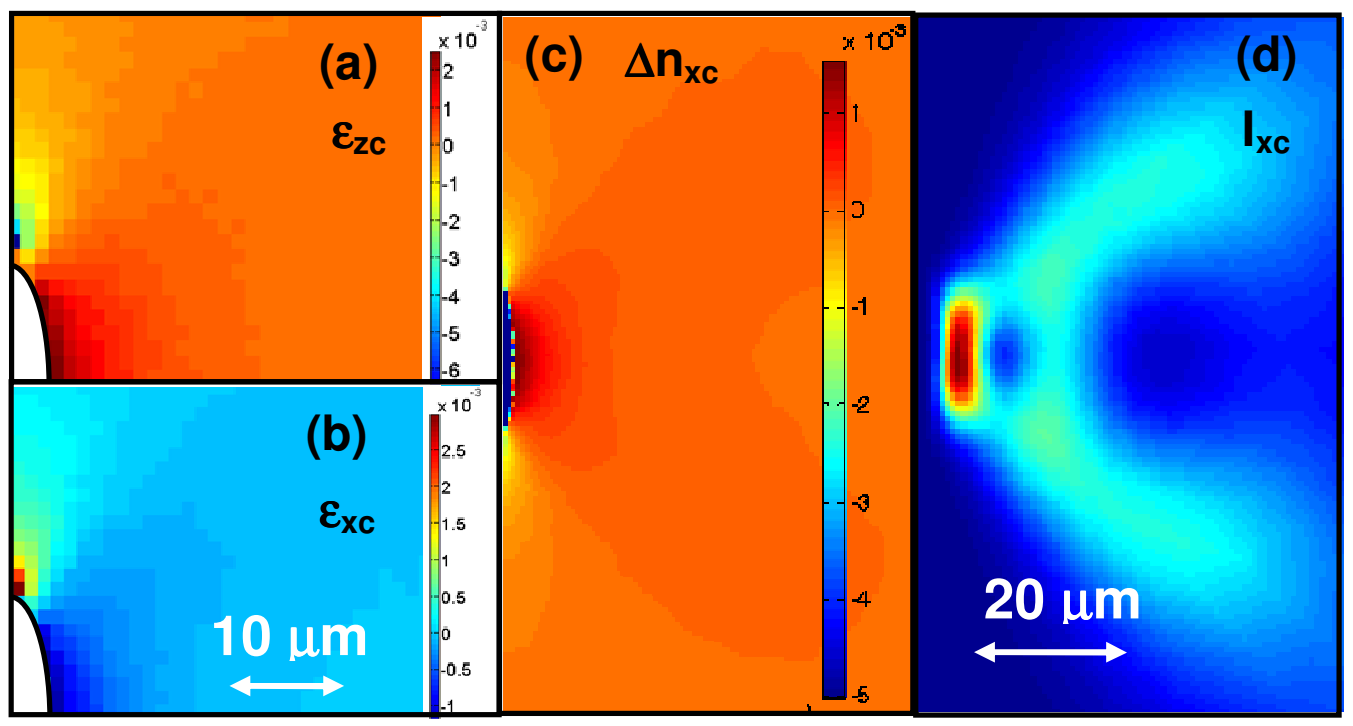

Figura 43. Resultados de expansión mecánica dada por $\alpha=\beta=0.003$ de elipse de radios $a=2 \mu \mathrm{m}$ y $b=9 \mu \mathrm{m}$ : (a) componente $z_{c}$ del campo de deformaciones, (b) componente $x_{c}$ del campo de deformaciones, (c) Variación de $\Delta \mathrm{n}_{\mathrm{xc}}$ aplicando relación piezo-óptica, (d) Distribución de intensidad guiada de polarización vertical $\mathrm{I}_{\mathrm{xc}}$. 


\section{Resultados}

\subsection{Estudio $\mu$-Raman bajo presión hidrostática de cristal de $\mathrm{Nd}: \mathrm{Mg}: \mathrm{LiNbO}_{3}$ (niobato de litio dopado con magnesio)}

El comportamiento espectral de los fonones bajo presión hidrostática está relacionado por la Teoría de Deformación Potencial con el comportamiento bajo deformaciones/presiones no-hidrostáticas (Capítulo 2). Por esto, en esta sección se estudia el comportamiento Raman bajo presión hidrostática del $\mathrm{Nd}: \mathrm{Mg}_{\mathrm{LiNbO}}$ y se lo compara con el del niobato de litio puro reportado en trabajos anteriores. Puntualmente, se analiza el corrimiento de los fonones transversales de las especies $A_{1}(T O)$ y $E(T O)$ para las configuraciones: $Y_{c}\left(Z_{c}-\right) Y_{c}$, denominada Configuración A, y, $Y_{c}\left(X_{c}-\right) Y_{c}$, denominada Configuración $B$. El guión del medio en estas expresiones indica que la polarización de colección (a la salida de la muestra) no fue seleccionada en el arreglo experimental. Por lo tanto, en la señal colectada se encuentran ambas polarizaciones $\left(x_{c}\right.$ y $z_{c}$ ) en ambas configuraciones estudiadas (A y B). Para analizar el corrimiento en número de onda de los fonones, en función de la presión hidrostática, se aplicó un proceso escalonado de carga hasta una presión de 6.5 Gpa y luego un proceso escalonado de descarga, utilizando el instrumento denominado Celda de Diamantes (DAC). El espectro Raman de la muestra es colectado en cada etapa.

\subsubsection{Rango completo de presión aplicada (hasta 6.3 GPa)}

En la Figura 44, se observan los espectros obtenidos para la configuración A y para la configuración B. En ambos casos, se rotulan de acuerdo a trabajos previos [QUI09, LEN07] los fonones identificados en los espectros. A su vez, se presentan, a modo de ejemplo, las funciones lorentzianas ajustadas en ambas configuraciones, para una presión de 0.7 Gpa en el caso de la Configuración A y para una presión de 6 Gpa en el caso de la Configuración B. En la Figura 44 se presenta el comportamiento del espectro Raman para valores de presiones que resulten representativos. De estos espectros, se puede observar, en general, el comportamiento previamente conocido para los fonones del niobato de litio puro [F|84]. Esto, se puede resumir en los siguientes ítems: 
- los fonones se desplazan espectralmente hacia valores mayores de número de onda con el aumento de presión hidrostática

- se aprecia una leve disminución de ancho medio en ellos cuando aumenta el valor de presión
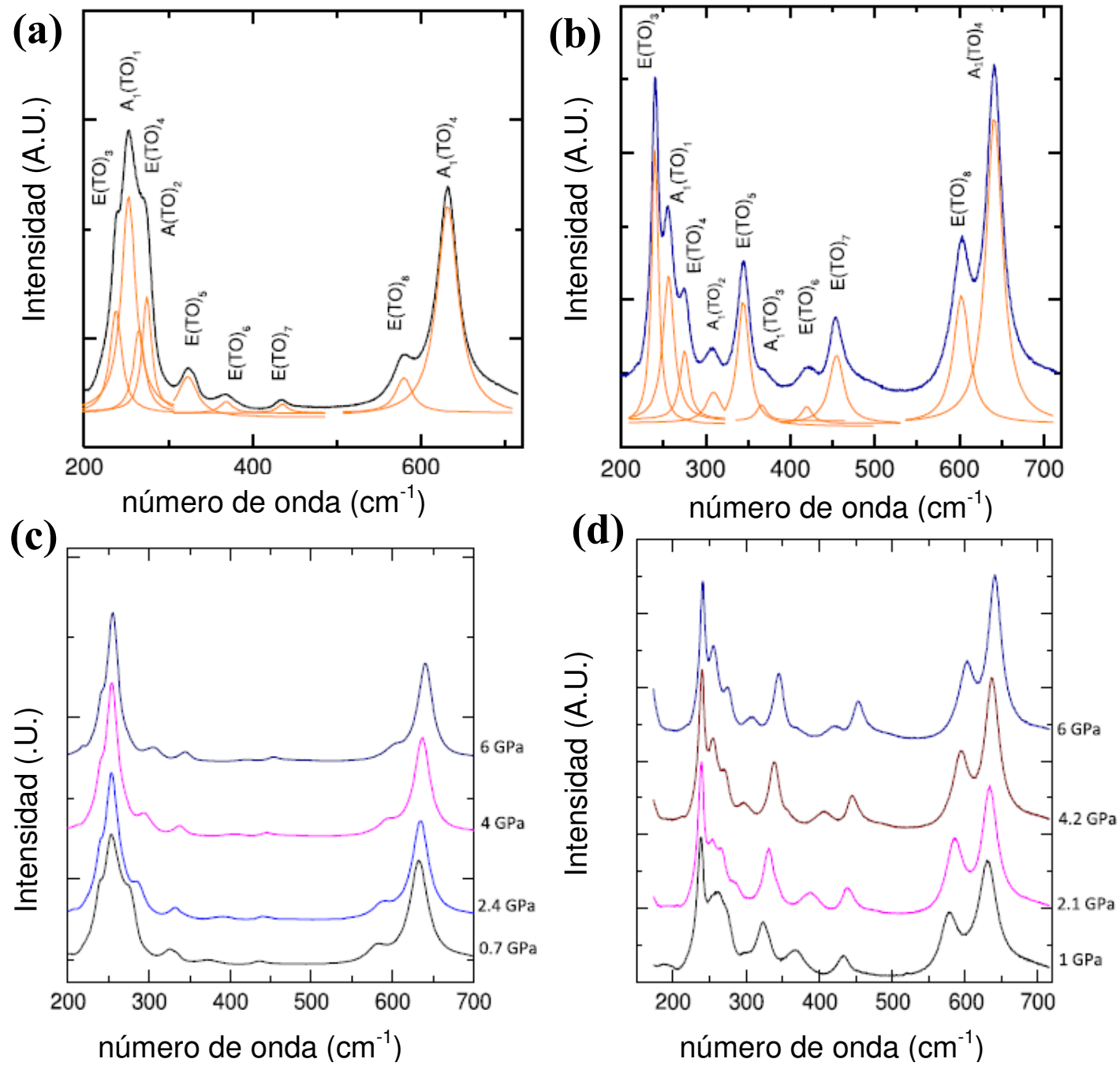

Figura 44. (a) Espectro Raman y ajuste de funciones Lorentzianas para la Configuración A (a $0.7 \mathrm{Gpa}$ ) y (b) para la Configuración B (a $6 \mathrm{GPa}$ ). (c) Espectros Raman para valores representativos de presión en la Configuración A y (d) en la Configuración B.

Una vez obtenidos los espectros, se ajustó una función Lorentziana a cada uno de los distintos fonones del espectro Raman para cada valor de presión. Para ello, se utilizó un algoritmo robusto generado por el Dr. A Goñi (Universidad Autónoma de Barcelona) que utiliza el método de mínimos cuadrados no-lineales "Marquardt" y se comenzó ajustando desde la presión máxima (6.3 Gpa) hacia los valores menores. Esto, se realizó de esta forma porque, en general, a bajas presiones los fonones están más solapados que a altas presiones. Utilizando el rango completo de presiones aplicadas (de 0 a $6.3 \mathrm{Gpa}$ ), se 
obtuvo el gráfico presentado en la Figura 45, donde se puede observar que el comportamiento resulta, en apariencia, aproximadamente lineal para este rango de presiones y para el rango espectral visualizado. Asumiendo una dependencia lineal entre la posición de los fonones y la presión hidrostática, se calcularon los coeficientes de presión o derivativas $(d \omega / d p)$ de la posición espectral respecto a la presión hidrostática asociadas a cada fonón y se presentan en la Tabla iv.

\begin{tabular}{llll}
\hline Fonón & $\begin{array}{c}\text { Número de } \\
\text { onda }\left(\mathrm{cm}^{-1}\right)\end{array}$ & $\begin{array}{c}\mathrm{d} \omega / \mathrm{dp}\left(\mathrm{cm}^{-1} / \mathrm{GPa}\right) \\
\mathrm{Nd}: \mathrm{Mg}: \mathrm{LiNbO}_{3}\end{array}$ & $\begin{array}{c}\mathrm{d} \omega / \mathrm{dp}\left(\mathrm{cm}^{-1} / \mathrm{GPa}\right) \\
\mathrm{LiNbO}_{3} \text { puro }\end{array}$ \\
\hline $\mathrm{E}(\mathrm{TO})_{3}$ & $@ 236$ & $0.6 \pm 0.1$ & $0.5 \pm 0.1$ \\
$\mathrm{~A}_{1}(\mathrm{TO})_{1}$ & $@ 253$ & $0.2 \pm 0.1$ & $\sim 0$ \\
$\mathrm{E}(\mathrm{TO})_{4}$ & $@ 261$ & $2.2 \pm 0.5$ & $0.4 \pm 0.1$ \\
$\mathrm{~A}_{1}(\mathrm{TO})_{2}$ & $@ 273$ & $5.7 \pm 0.1$ & $5.2 \pm 0.1$ \\
$\mathrm{E}(\mathrm{TO})_{5}$ & $@ 323$ & $3.5 \pm 0.1$ & $3.4 \pm 0.2$ \\
$\mathrm{~A}_{1}(\mathrm{TO})_{3}$ & $@ 330$ & $7.0 \pm 0.1$ & $2.1 \pm 0.1$ \\
$\mathrm{E}(\mathrm{TO})_{6}$ & $@ 368$ & $8.8 \pm 0.3$ & $8.3 \pm 0.3$ \\
$\mathrm{E}(\mathrm{TO})_{7}$ & $@ 436$ & $3.5 \pm 0.1$ & $3.5 \pm 0.1$ \\
$\mathrm{E}(\mathrm{TO})_{8}$ & $@ 578$ & $4.3 \pm 0.1$ & $4.2 \pm 0.3$ \\
$\mathrm{~A}_{1}(\mathrm{TO})_{4}$ & $@ 631$ & $1.6 \pm 0.1$ & $1.5 \pm 0.1$ \\
\hline
\end{tabular}

Tabla iv. Coeficientes de presión $d \omega / d p$ para $\mathrm{Nd}: \mathrm{Mg}: \mathrm{LiNbO}_{3}$ y $\mathrm{LiNbO}_{3}$

En la misma, también se incluyen aquellos valores reportados por Filho y sus colaboradores [Fl84] para los coeficientes del niobato de litio puro. Comparando ambos resultados, se puede observar que para los fonones $E(T O)_{3}, A_{1}(T O)_{1}, E(T O)_{5}, E(T O)_{6}$, $\mathrm{E}(\mathrm{TO})_{7}, \mathrm{E}(\mathrm{TO})_{8}$ y $\mathrm{A}_{1}(\mathrm{TO})_{4}$, los coeficientes de presión $(d \omega / d p)$ coinciden entre el $\mathrm{LiNbO}_{3}$ y el $\mathrm{Nd}: \mathrm{Mg}: \mathrm{LiNbO}_{3}$, dentro de las incertezas experimentales. Para los fonones $\mathrm{A}_{1}(\mathrm{TO})_{2}$ y $\mathrm{A}_{1}(\mathrm{TO})_{3}$, se observa un incremento de los coeficientes de presión $(d \omega / d p)$ en un $15 \%$ y $200 \%$ en el $\mathrm{Nd}: \mathrm{Mg}: \mathrm{LiNbO}_{3}$, con respecto al $\mathrm{LiNbO}_{3}$ puro. A su vez, para el fonón $\mathrm{E}(\mathrm{TO})_{4}$ se observa un incremento en un $450 \%$ del coeficiente de presión por efecto del dopaje con Nd:Mg.

Para los fonones en los que el coeficiente de presión varía por efecto del dopaje con $\mathrm{Nd}: \mathrm{Mg}\left(\mathrm{A}_{1}(\mathrm{TO})_{2}, \mathrm{~A}_{1}(\mathrm{TO})_{3}\right.$ y $\left.\mathrm{E}(\mathrm{TO})_{4}\right)$, se reportó en trabajos previos [LEN07,MOU00,KIM97] también una variación en su posición espectral a presión ambiente de estos fonones, por efecto de dichos dopantes. 


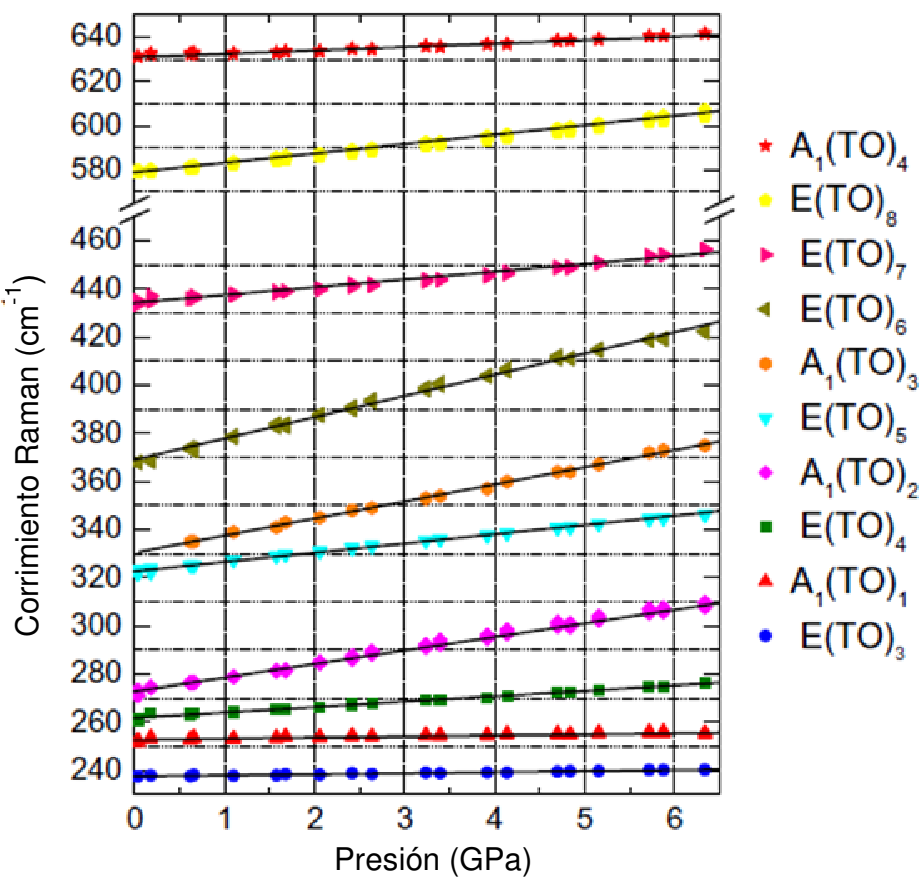

Figura 45. Posición espectral de los fonones Raman medidos en una muestra de $\mathrm{Nd}: \mathrm{Mg}: \mathrm{LiNbO}_{3}$ en función de presión para el rango completo estudiado ( 0 a 6.3 Gpa).Las incertezas son menores que que el tamaño de los símbolos.

\subsubsection{Comportamiento de fonones $A_{1}(T O)$ en rango completo y reducido (hasta $2 \mathrm{Gpa}$ ) de presión}

En particular, este trabajo de tesis apunta al estudio del corrimiento espectral de los fonones $A_{1}(T O)$ en guías de onda. Estos fonones se utilizan porque son fácilmente detectables y se pueden separar del resto del espectro Raman mediante la configuración adecuada. Los corrimientos en número de onda de los fonones $A_{1}$ (TO) en guías de onda fabricadas con láser de femtosegundos, han sido reportados en trabajos previos. Este corrimiento espectral (en número de onda) es como máximo, aproximadamente $0.5 \mathrm{~cm}^{-1}$ para el fonón $\mathrm{A}_{1}(\mathrm{TO})_{4}$. Asumiendo una relación lineal entre el corrimiento espectral y la presión hidrostática (aunque en la guía de onda, la presión residual no sea hidrostática), dada por el coeficiente de presión obtenido para el fonón $\mathrm{A}_{1}(\mathrm{TO})_{4}$ en la sub-sección previa $\left(1.6 \mathrm{~cm}^{-1} / \mathrm{Gpa}\right)$, obtenemos un valor de presión hidrostática de $0.4 \mathrm{Gpa}$. Por lo tanto, el rango completo de presiones hidrostáticas (hasta $6.3 \mathrm{Gpa}$ ) estudiado en la subsección previa, es muy superior al detectado en guías de onda fabricadas con láser de femtosegundos.

Para observar en forma más detallada el comportamiento de estos fonones a bajas presiones se presenta la Figura 46. En esta figura, se puede observar que la 
aproximación lineal utilizada en el ajuste anterior, no es del todo acertada principalmente para los fonones $A_{1}(T O)_{1}$ y $A_{1}(T O)_{4}$ (Figura 46). En ellos se observa un comportamiento no lineal en función de la presión. Por el contrario, en los fonones $A_{1}(T O)_{2}$ y $A_{1}(T O)_{3}$ se observa un comportamiento de mayor linealidad (Figura 46). En esta figura, se presenta una línea de puntos recta para facilitar la visualización de este comportamiento.

En lo que respecta a la carga y descarga, se observa que en los fonones $A_{1}(T O)_{2}$ y $\mathrm{A}_{1}(\mathrm{TO})_{3}$ son coincidentes. Lo mismo ocurre con el comportamiento de estos fonones para las distintas configuraciones.

Por otro lado, para los fonones $A_{1}(T O)_{1}$ y $A_{1}(T O)_{4}$ se observó una diferencia de la posición espectral entre la carga y la descarga: en esta última, en general, la posición espectral de estos fonones está desplazada hacia mayores valores de número de onda. En estos modos de vibración, también se observó un corrimiento de la posición espectral para las distintas configuraciones analizadas ( $A$ y $B$ ). Esto puede ser generado por solapamiento con modos de vibración longitudinales en el caso del fonón $A_{1}(T O)_{4}$ (que son de considerable menor intensidad [BER09]), ó por solapamiento con otros fonones transversales (de especie $\mathrm{E}(\mathrm{TO})$ ). De todas formas, se puede ver en las figuras correspondientes, que las distintas pendientes (que es lo que interesa en el presente estudio) son aproximadamente coincidentes entre ambas configuraciones.
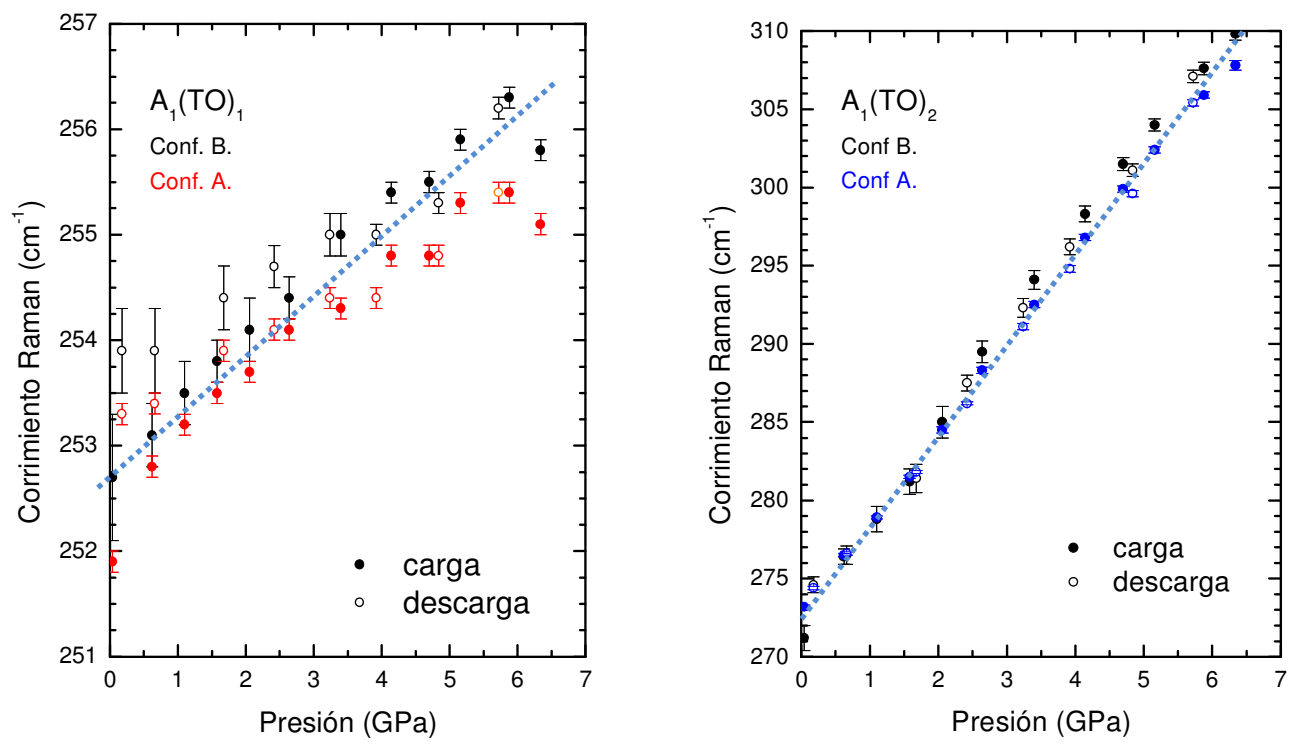

Figura 46. Posición espectral de fonones $A_{1}(T O)_{i}$ en función de presión hidrostática. 

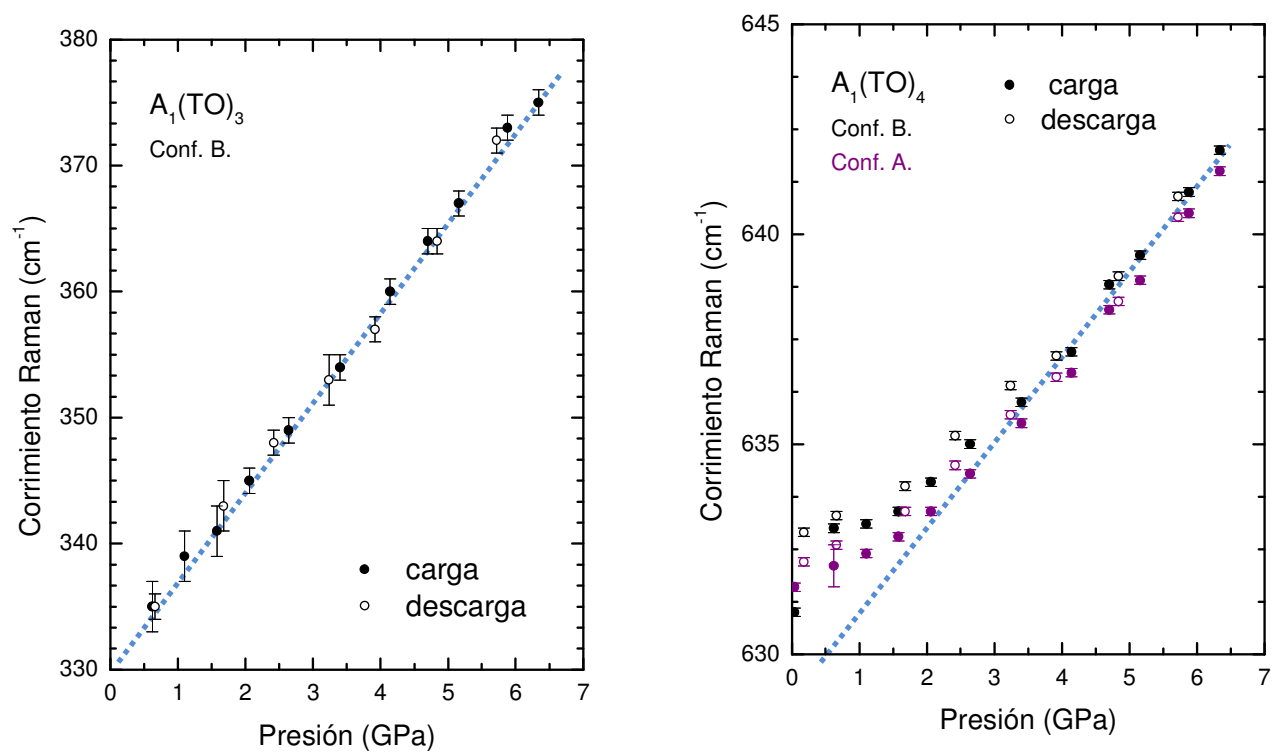

Figura 46.Posición espectral de fonones $A_{1}(T O)_{i}$ en función de presión hidrostática.

Como la dependencia entre la posición de los fonones y la presión hidrostática no es estrictamente lineal, se acotó el rango de presiones limitándose a presiones relativamente bajas (hasta $2.5 \mathrm{Gpa}$ ), y, nuevamente se ajustó linealmente de tal forma de obtener coeficientes de presión $(d \omega / d p)$ más adecuados para el estudio de las guías de onda. Los puntos utilizados son en condición de carga.

Los ajustes efectuados se presentan en la Figura 47. Los coeficientes $d \omega / d p$ resultantes se presentan en la Tabla iii. En ella, se puede ver que los fonones $A_{1}(T O)_{2}$ y $A_{1}(T O)_{3}$, tienen un $d \omega / d p$ muy similar en ambos dominios. Mientras que el fonón $A_{1}(T O)_{1}$ observa un aumento de este coeficiente y el fonón $\mathrm{A}_{1}(\mathrm{TO})_{4}$ una disminución del mismo para el dominio reducido (hasta $2.5 \mathrm{Gpa}$ ). En la siguiente sección, se utilizarán los valores de $d \omega / d p$ obtenidos utilizando el dominio reducido para analizar, como primera aproximación, los corrimientos Raman medidos en las guías de onda.

De todas formas, se considera importante observar en la Figura 47 que a baja carga (menor a $0.5 \mathrm{GPa}$ ) existe una importante dispersión en los datos, en particular para los fonones $A_{1}(T O)_{1}$ y $A_{1}(T O)_{4}$. Esta región se marca con línea de puntos en dicha Figura. Lamentablemente, este es el rango de variación de número de onda en el que se encuentran los corrimientos Raman medidos en las guías de onda fabricadas con láser de femtosegundos [RO09]. Por lo tanto, el coeficiente $d \omega / d p$ para este rango podría diferir del determinado. Se estima que el método convencional utilizado (compresión por celda de diamantes), no es apropiado para medir corrimientos Raman a bajas presiones hidrostáticas y que para aplicarlo con esta finalidad debería ser adaptado. 

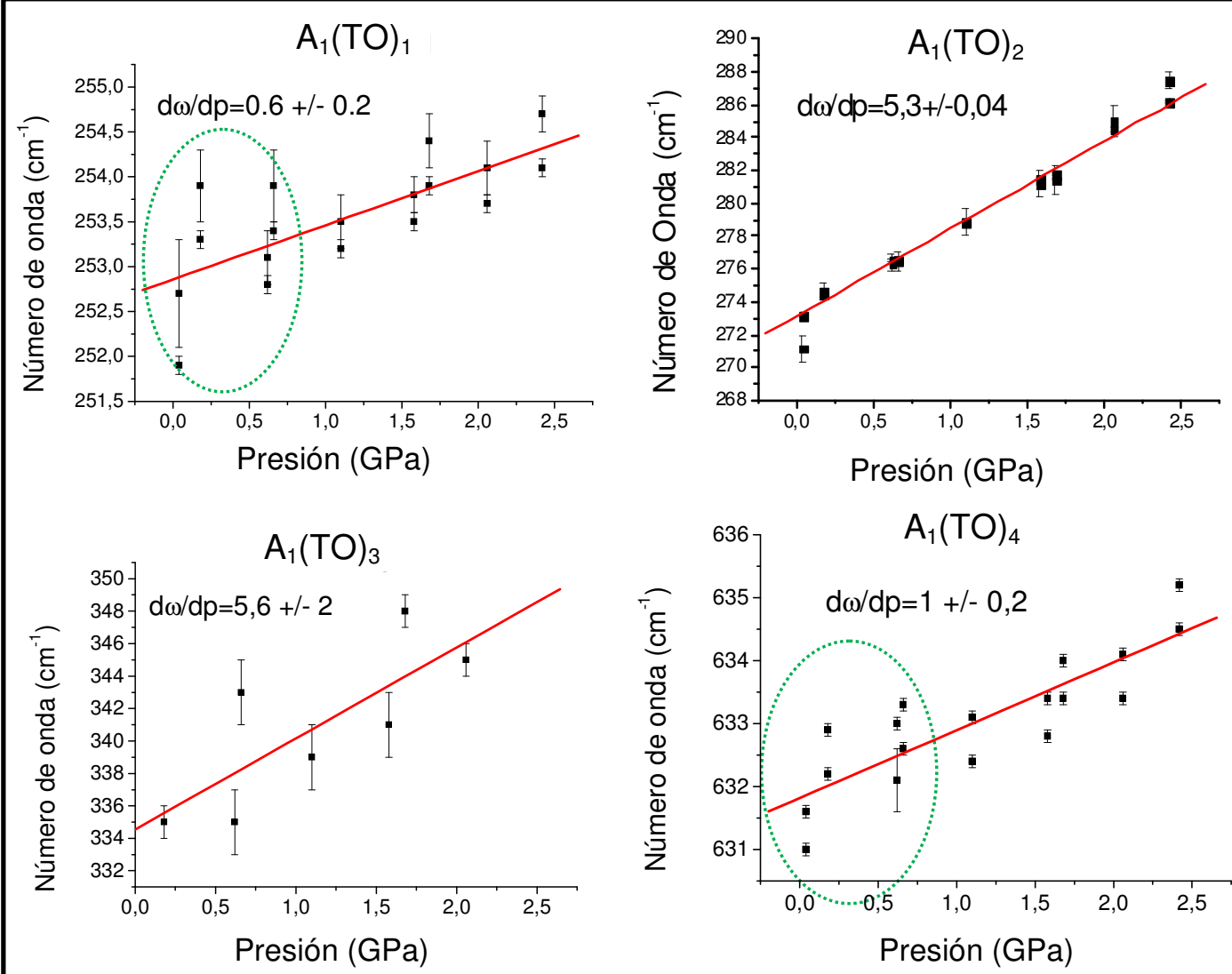

Figura 47. Ajuste lineal de la posición de los fonones para rango reducido de presiones (hasta 2 Gpa)

\begin{tabular}{|c|c|c|c|c|}
\hline Fonón & $\mathrm{A}_{1}(\mathrm{TO})_{1}$ & $\mathrm{~A}_{1}(\mathrm{TO})_{2}$ & $\mathrm{~A}_{1}(\mathrm{TO})_{3}$ & $\mathrm{~A}_{1}(\mathrm{TO})_{4}$ \\
\hline $\begin{array}{c}\boldsymbol{d} \boldsymbol{\omega} \boldsymbol{d} \boldsymbol{p}\left(\mathrm{cm}^{-1} / \mathrm{Gpa}\right) \\
\text { hasta } 6.3 \mathrm{Gpa}\end{array}$ & $0.2+/-0.1$ & $5.7+/-0.1$ & $7.0+/-0.1$ & $1.6+/-0.1$ \\
\hline $\begin{array}{c}\boldsymbol{d} \boldsymbol{\omega} \boldsymbol{d} \boldsymbol{p}\left(\mathrm{cm}^{-1} / \mathrm{Gpa}\right) \\
\text { hasta } 2 \mathrm{Gpa}\end{array}$ & $0.7+/-0.1$ & $5.3+/-0.1$ & $5.6+/-2.0$ & $1.0+/-0.2$ \\
\hline
\end{tabular}

Tabla v. Valores de $\mathrm{d} \omega / \mathrm{dp}$ para dominio completo y dominio reducido de presiones

\subsubsection{Resumen}

En esta sub-sección se estudió, en forma global, el comportamiento del espectro Raman de una muestra de $\mathrm{Mg}: \mathrm{Nd}: \mathrm{LiNbO}_{3}$ bajo presión hidrostática y se lo comparó con el $\mathrm{LiNbO}_{3}$ puro. No se encontraron diferencias considerables en los valores de $d \omega / d p$, a excepción de los fonones $\mathrm{A} 1(\mathrm{TO})_{2}, \mathrm{~A} 1(\mathrm{TO})_{3}$ y $\mathrm{E}(\mathrm{TO})_{4}$, en los que se observaron un incremento del $15 \%, 200 \%$ y $450 \%$, respectivamente, por presencia de los dopantes mencionados. Luego, estudiando en detalle los fonones $A_{1}(T O)$ se observó que la relación entre la 
presión hidrostática y el corrimiento espectral no es estrictamente lineal. Por tal motivo, se utilizó un dominio reducido (hasta $2.5 \mathrm{Gpa}$ ) para determinar nuevos coeficientes lineales de presión ( $\mathrm{d} \omega / \mathrm{dp}$ ), que sean más apropiados para analizar guías de onda fabricadas con láser de femtosegundos. De todas formas, se observó una importante dispersión en los datos de corrimiento espectral vs presión hidrostática para bajas presiones (hasta $1 \mathrm{GPa}$ ). Este es el rango en el que se encuentran las medidas de corrimiento Raman en las guías de onda fabricadas con láser de femtosegundos.

\subsection{Estudio micro-Raman y de intensidad guiada de guias de onda fabricadas con laser de femtosegundos}

En los estudios pioneros utilizando $\mu$-Raman en guías de onda fabricadas con láser de femtosegundos, la motivación principal fue detectar variaciones del espectro Raman para inferir modificaciones estructurales y "presiones" localizadas de forma cualitativa, tanto en la zona de guiado de luz como en la zona de interacción directa. En el presente trabajo se decide avanzar hacia la obtención de información más precisa sobre el estado de deformación del material en la zona de guiado. Para ello, en esta sección, se presentan los resultados obtenidos de la comparación y ajuste de medidas experimentales de corrimientos $\mu$-Raman e intensidad guiada, con el modelo numérico desarrollado en el Capitulo 4. Como se mencionó en el mismo, este modelo elástico fue utilizado por otros autores [BUR07, WILL05] para explicar el origen de guías de onda de este tipo, tanto en $\mathrm{LiNbO}_{3}$ como en $\mathrm{SiO}_{2}$. Con lo cual, el mismo se consideró de potencial utilidad para estudiar las deformaciones en este tipo de guía de onda, como así también, obtener valores aproximados de las constantes de deformación potencial del $\mathrm{LiNbO}_{3}$, por intermedio de la Teoría de Deformación Potencial Lineal ${ }^{4}$.

\subsubsection{Guías de onda estudiadas}

Las modificaciones inducidas con el láser de femtosegundos en una muestra de $\mathrm{Mg}: \mathrm{Nd}: \mathrm{LiNbO}_{3}$ que se estudiaron utilizando $\mu$-Raman y distribución de intensidad propagada, se presentan en la Figura 48.

\footnotetext{
${ }^{4}$ Marco teórico que relaciona las deformaciones con los corrimientos espectrales de los fonones del material (Capítulo 4).
} 


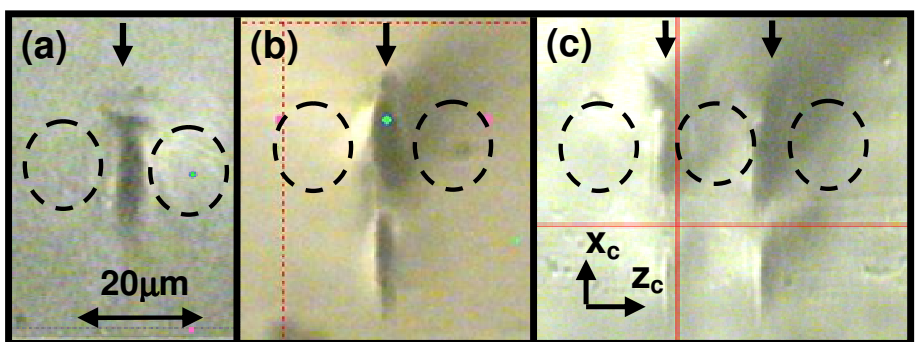

Figura 48. Imágenes microscópicas de la sección transversal de: (a) modificación A: simple sin filamentación, (b) modificación B: guías de modificación simple con "filamento", (c) modificación C: guías de modificación doble con "filamento". Las flechas indican la dirección de incidencia del láser de fs. Las curvas con líneas de a trazos indican la zona principal de guiado de luz. Todas las figuras están en la misma escala, (d) esquema de fabricación de las modificaciones $\mathrm{A}$, B y $\mathrm{C}$ con láser de femtosegundos.

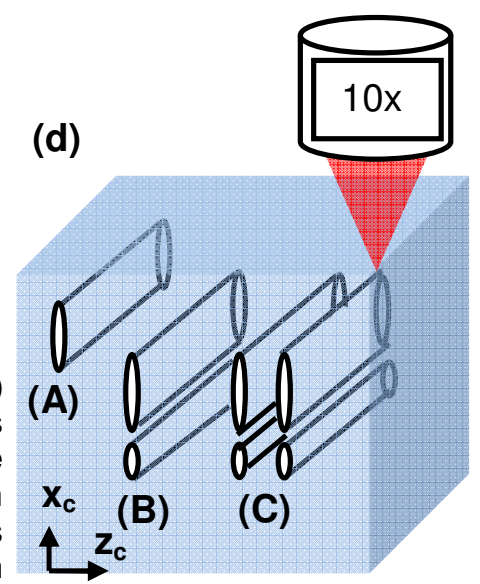

Las tres modificaciones (A, B y C) fueron fabricadas sobre la misma muestra "corte $\mathrm{x}$ " de $\mathrm{Nd}: \mathrm{Mg}: \mathrm{LiNbO}_{3}$ utilizando la técnica de escritura láser presentada en el Capítulo 3 y como se detalla en la Figura 48(d). Se utilizaron los siguientes parámetros de procesado: objetivo de microscopio de 10x (NA=0.25), velocidad desplazamiento de $30 \mu \mathrm{m} / \mathrm{s}$, energía de pulso de $1 \mu \mathrm{J}$ y duración de pulso de 120 fs. Para generar la modificación A se enfocó el láser de escritura $80 \mu \mathrm{m}$ por debajo de la superficie, mientras que las modificaciones B y C se enfocaron a $150 \mu \mathrm{m}$ por debajo de la misma. Esta diferencia es la que genera una geometría diferente en dichas modificaciones: se observa una "filamentación" (o segunda modificación) producida por la propagación no lineal para las guías fabricadas a mayor profundidad (Más información en Capítulo 2). Estas estructuras, fueron fabricadas por L. Roso y colaboradores en el grupo de Óptica ("Fotón Charro") de la Universidad de Salamanca [R009_2]. Todas las estructuras generadas permitieron el acople de luz principalmente en las adyacencias de las modificaciones principales, estas zonas se marcan con curvas de línea de trazos en la Figura 48. En las estructuras B y C, también se visualizó acople de luz, aunque de menor intensidad, en las zonas secundarias (filamentaciones) ubicadas debajo de las modificaciones principales.

\subsubsection{Análisis unidimensional de guía de onda}

El principal objetivo de este primer análisis es evaluar el desempeño del modelo numérico para ajustar un mapeo lineal de corrimiento Raman de los fonones de especie $\mathrm{A}_{1}$ (TO) en una guía de onda fabricada con láser de femtosegundos. A su vez, se utilizan los coeficientes de presión $(d \omega / d p)$ y dicho mapeo lineal para determinar, en una primera aproximación, las características de la expansión (relación entre $\alpha$ y $\beta$ ) que generó la guía de onda estudiada. También se obtuvieron, en una primera aproximación, valores 
de los parámetros del modelo numérico $(a, b, \alpha$ y $\beta)$ y de las constantes de deformación potencial e y $f$.

Como punto de partida para el análisis unidimensional, se utilizó un mapeo lineal $\mu$-Raman en configuración $\mathrm{Y}(\mathrm{ZZ}) \mathrm{Y}$ en las inmediaciones de la modificación $\mathrm{A}$, que se presenta en la Figura 49 (a). El mapeo fue realizado de a intervalos de a $0.5 \mu \mathrm{m}$ a lo largo de la trayectoria b'b". Esta trayectoria se esquematiza también en la Figura 49(a). En la Figura 49(b), se presenta la intensidad guiada a la derecha de la estructura ( sobre la trayectoria $b^{\prime} b^{\prime \prime}$ ) y en la Figura 49 (c) se presenta la geometría adoptada para la elipse del modelo numérico en esta primera etapa. En la Figura 50, se presenta un espectro Raman en un punto arbitrario de la trayectoria b'b'. La medición de la intensidad guiada y el mapeo $\mu$-Raman sobre esta trayectoria, fueron realizadas por A. Ródenas y colaboradores [RO09].

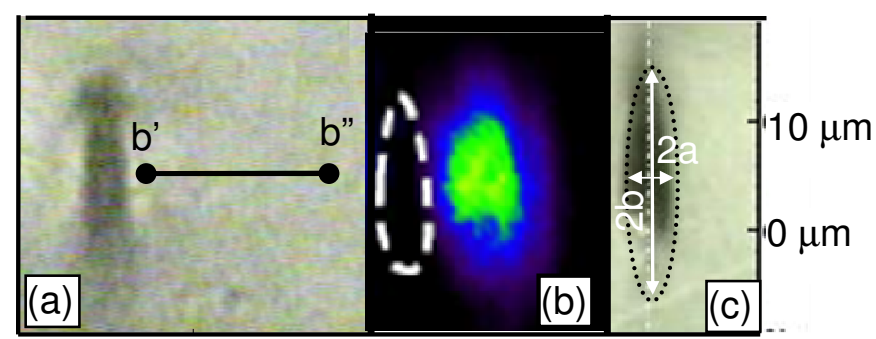

Figura 49. (a) Esquema de trayectoria b' b" analizada, (b)luz guiada acoplada en la guía adyacente a la modificación,(c) tamaño de elipse modelada en el primer cálculo (radios a y b). Todas las imágenes están en la misma escala.

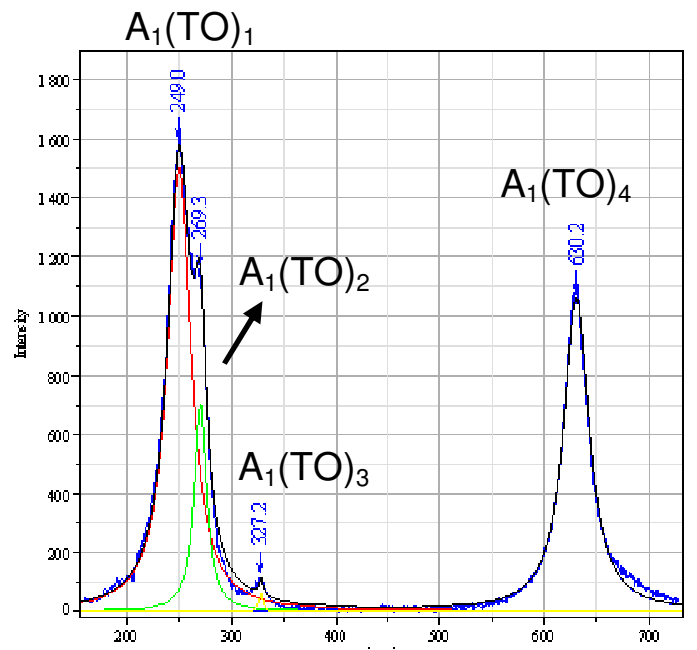

Figura 50. Espectro Raman en un punto arbitrario de la trayectoria b'b" en configuración $\mathrm{Y}(\mathrm{ZZ}) \mathrm{Y}$ en programa comercial LabSpec®.

El modelo numérico fue implementado, en este análisis unidimensional, utilizando el código 1.m que se presenta en el Anexo B. 
Se pueden definir tres etapas asociadas a este análisis:

Etapa I: Primeramente, se ajustaron las deformaciones $\varepsilon_{\mathrm{xc}}$ y $\varepsilon_{\mathrm{zc}}$ obtenidos en el modelo numérico (para parámetros pre-establecidos), a los corrimientos de los fonones Raman $A_{1}(\mathrm{TO})$ sobre la trayectoria b'b". Los parámetros pre-establecidos aplicados en el modelo numérico corresponden a: una elipse de las dimensiones a y $b$ obtenidas de la Figura 49 (c) y una expansión (dada por $\alpha$ y $\beta$ ) de acuerdo a la reportada en la literatura.

Etapa II: A continuación, se evaluaron 294 combinaciones de parámetros $a, b$ y $\beta / \alpha$, analizando los 294 conjuntos de valores numéricos de $d \omega / d p_{\text {num }}$ asociados a cada fonón. Luego, se utilizaron desigualdades entre valores experimentales de $d \omega / d p$ para descartar las combinaciones de parámetros que no sean físicamente adecuadas.

Etapa III: Un tercer y último paso, consistió en minimizar la diferencia entre los valores numéricos y experimentales de $d \omega / d p$. Los valores iniciales utilizados en esta minimización fueron extraídos de los valores asumidos como físicamente probables en el paso anterior.

\section{Descripción de etapas I, II y III:}

\section{Etapa I}

Para un primer ajuste, se definieron los siguientes radios iniciales de la elipse a expandir: $\mathrm{a}=2 \mu \mathrm{m}$ y $\mathrm{b}=10 \mu \mathrm{m}$, obtenidos de la imagen óptica de la sección transversal de la modificación generada con el láser de escritura (Figura 49 (c)).

Despreciando cambios de polarización en el material modificado, es decir que la disminución del índice de refracción en la zona de interacción directa, se asume producida solamente por una variación de volumen, se puede obtener el cambio de volumen de la zona modificada en función de la variación de índice de refracción (Ecuación 69) [BUR07,VO10].

$$
\frac{\Delta V}{V}=-0.5 \Delta n
$$

A su vez, considerando la variación de índice de refracción generalmente medida en la zona de modificación estructural para este tipo de guías de onda [BUR07, RO09, BIA13]: $\Delta \mathrm{n}=-1 \times 10^{-2}$ y utilizando la Ecuación 69 , se puede obtener una variación de volumen de la elipse $\Delta \mathrm{V} / \mathrm{V}=5 \times 10^{-3}$. Despreciando efectos dinámicos y de contacto entre el cuerpo que se expande y el medio que se opone a ello, se definió para esta etapa, $\Delta \mathrm{a} / \mathrm{a}=\Delta \mathrm{b} / \mathrm{b}$. Esta aproximación también se utilizó en trabajos previos [BUR07]. Es decir que, en esta primera aproximación, la elipse sufre una expansión proporcional. Por lo tanto, utilizando la Ecuación 70 y $\Delta \mathrm{V} / \mathrm{V}=5 \times 10^{-3}$ se puede obtener $\alpha=0.0025(\Delta \mathrm{a} / \mathrm{a})$ y $\beta=0.0025(\Delta \mathrm{b} / \mathrm{b})$.

$$
\frac{\Delta V}{V}=\frac{\Delta a}{a}+\frac{\Delta b}{b}
$$




\section{Procedimiento}

Utilizando la ecuación $\Delta \omega=e \varepsilon_{\mathrm{zc}}+f \varepsilon_{\mathrm{xc}}$ (Teoría de Deformación Potencial Lineal-Capítulo 3) y el método de mínimos cuadrados, se ajustaron las deformaciones $\varepsilon_{z c}$ y $\varepsilon_{x c}$ resultantes de la expansión propuesta en el modelo numérico a los valores experimentales de los corrimientos Raman $(\Delta \omega)$ para los fonones $A_{1}(T O)$. En este procedimiento, los parámetros de ajuste son las Constantes de Deformación Potencial e y $f$ asociadas a cada fonón. Es preciso remarcar que al momento de realizar este trabajo no se encontraban reportados valores de estas constantes del $\mathrm{LiNbO}_{3}$.

Como resultado del ajuste, se obtuvieron valores "numéricos" para las constantes de deformación potencial $\left(e\right.$ y $f$ ) y los coeficientes de presión hidrostática de $d \omega / \mathrm{dp}_{\text {num }}$ (iguales a $2 e^{\prime}+f^{\prime 5}$ ). Estos resultados se presentan en la Figura 51 y en la Tabla vi.

${ }^{5}$ De acuerdo a la Teoría de Deformación Potencial, los valores de e'y f'dependen unicamente de los valores de e y $f$, y de las constantes elásticas para un determinado material (Capítulo 4). 

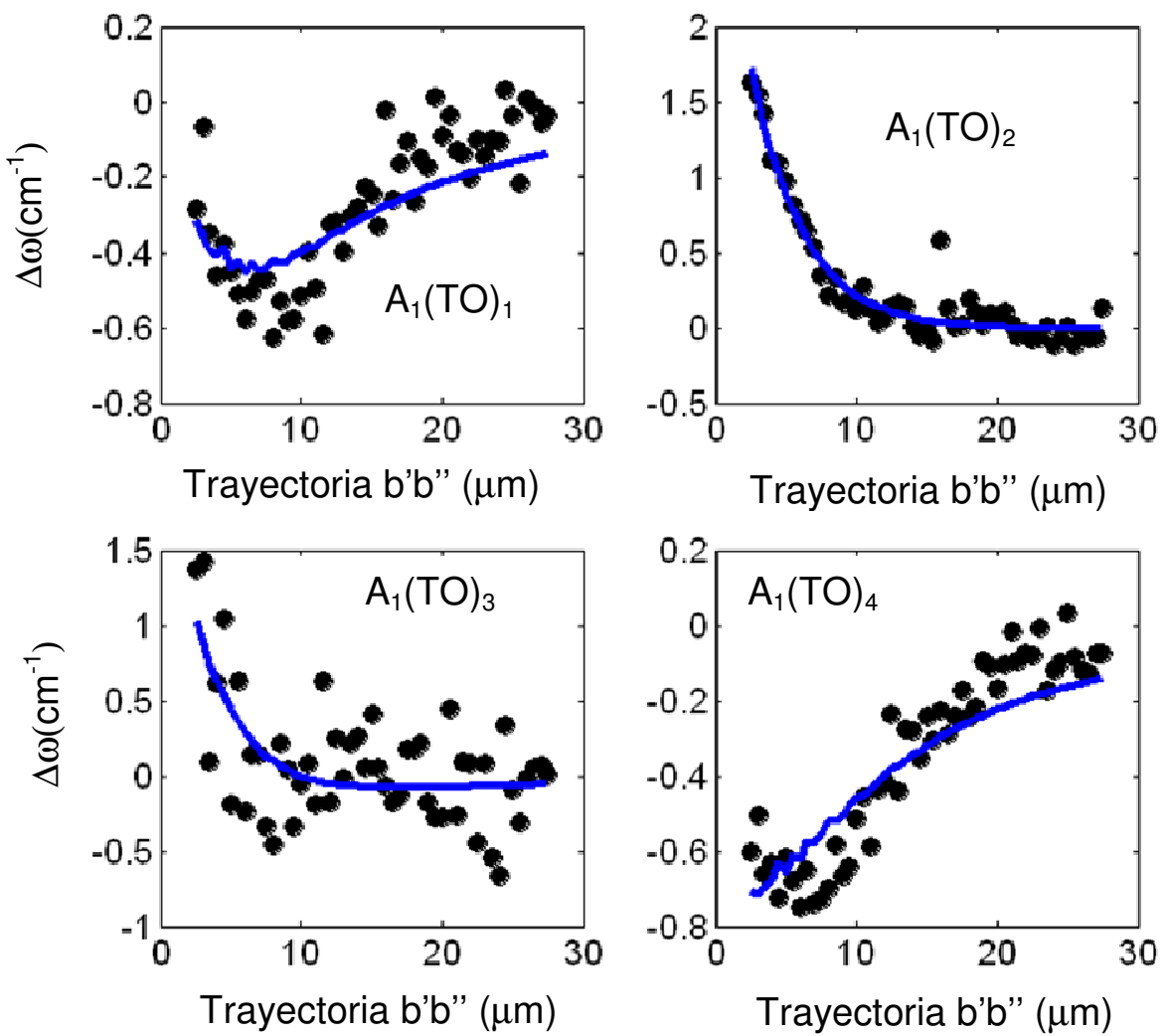

Figura 51. Ajuste de los corrimientos en número de onda de los fonones $A_{1}(T O)_{1}$ a $A_{1}(\mathrm{TO})_{4}$ a lo largo de la trayectoria $b^{\prime} b$ ", las medidas experimentales se presentan en círculos negros y los ajustes con línea azul

\begin{tabular}{|l|l|l|l|c|l|}
\hline Fonón & $\mathrm{e}$ & $\mathrm{f}$ & E.C.M. & $d \omega / d p_{\text {num }}\left[\mathrm{cm}^{-1} / \mathrm{GPa}\right]$ & $d \omega / d p_{\text {exp }}{ }^{* 6}$ \\
\hline $\mathrm{A}_{1} \mathrm{TO}_{1}$ & -2500 & -4600 & 0.1 & 26 & 0.7 \\
\hline $\mathrm{A}_{1} \mathrm{TO}_{2}$ & -440 & -2200 & 0.1 & 8 & 5.5 \\
\hline $\mathrm{A}_{1} \mathrm{TO}_{3}$ & -1200 & -3229 & 0.3 & 15 & 6.6 \\
\hline $\mathrm{A}_{1} \mathrm{TO}_{4}$ & -2500 & -4300 & 0.1 & 26 & 1.1 \\
\hline
\end{tabular}

Tabla vi.Valores obtenidos del ajuste: constantes de deformación potencial (e $y f$ ), errores cuadráticos medios de los distintos ajustes (ECM) y coeficiente numérico bajo presión hidrostática d $\omega / \mathrm{dp} \mathrm{p}_{\text {num }}$

${ }^{6}$ Los valores de dw/dp corresponden a un rango de presión de 0 a 2 Gpa. 

femtosegundos en niobato de litio

\begin{tabular}{|c|c|c|}
\hline Experimental & Numérico & ¿Coinciden? \\
\hline$d \omega / d p_{1}<d \omega / d p_{2}$ & $d \omega / d p_{1}>d \omega / d p_{2}$ & $N O$ \\
\hline$d \omega / d p_{4}<d \omega / d p_{2}$ & $d \omega / d p_{4}>d \omega / d p_{2}$ & $N O$ \\
\hline$d \omega / d p_{1}<d \omega / d p_{3}$ & $d \omega / d p_{1}>d \omega / d p_{3}$ & $N O$ \\
\hline$d \omega / d p_{4}<d \omega / d p_{3}$ & $d \omega / d p_{4}>d \omega / d p_{3}$ & $N O$ \\
\hline$d \omega / d p_{2}<d \omega / d p_{3}$ & $d \omega / d p_{2}<d \omega / d p_{3}$ & $S I$ \\
\hline$d \omega / d p_{1}<d \omega / d p_{4}$ & $d \omega / d p_{1}<d \omega / d p_{4}$ & $N O$ \\
\hline
\end{tabular}

Tabla vii. Concordancia entre las desigualdades asociadas a los distintos valores de $\mathrm{d} \omega \mathrm{dp}$ num $\mathrm{y}$ aquellos de $\mathrm{d} \omega / \mathrm{dp} \mathrm{p}_{\text {exp }}$. Por ejemplo, si $\left(d \omega / d p_{\text {exp }} 2>d \omega / d p_{\text {exp }} 1\right) \&\left(d \omega / d p_{\text {num }} 2>d \omega / d p_{\text {num }} 1\right)$.

El ajuste del modelo numérico a los corrimientos de los fonones $A_{1}(T O)$ en la trayectoria analizada (Figura 51 y Tabla vi), se puede considerar aceptable. En general el Error Cuadrático Medio (ECM) es aproximadamente 0.1 , salvo para el fonón $A_{1}(T O)_{3}$ para el cual $E C M=0.3$. Se puede ver en la Figura 51, que para este fonón la medida del corrimiento de número de onda tiene una importante dispersión. Por otro lado, los valores numéricos de $\mathrm{d} \omega / \mathrm{dp}_{1}$ y $\mathrm{d} \omega / \mathrm{dp}_{4}$ son mayores en un orden de magnitud ó más, a los correspondientes $d \omega / d p_{\text {exp }}$. En cambio, para el fonón $A_{1}(T O)_{2}$ y $A_{1}(T O)_{3}$ se observa que los valores de $\mathrm{d} \omega / \mathrm{dp}$ num y los $\mathrm{d} \omega / \mathrm{dp}$ exp tienen menor discrepancia. Esta característica indica que, en el ajuste efectuado, los fonones no poseen un comportamiento homogéneo. Ya que se puede interpretar que la expansión fue sub-estimada, en un orden de magnitud ó más, si se tienen en cuenta los fonones $A_{1}(T O)_{1}$ y $A_{1}(T O)_{4}$. Ó bien, la misma tuvo un orden de magnitud adecuado, según los fonones $A_{1}(T O)_{2}$ y $A_{1}(T O)_{3}$. $A$ su vez, se puede ver en la Tabla vii que la desigualdad entre los valores de $\mathrm{d} \omega / \mathrm{dp}$ num se corresponde con la desigualdad de los valores de $d \omega / d p_{\text {exp }}$, solamente para una de las seis desigualdades posibles. Siendo esta, una desigualdad débil por la pequeña diferencia entre los correspondientes $d \omega / d p$. Como consecuencia, se consideró que

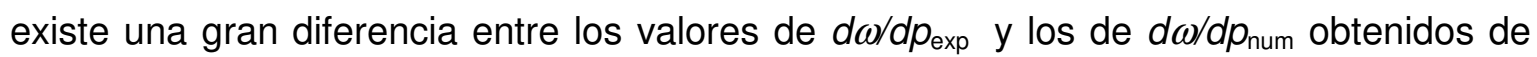
este ajuste. Por consiguiente, se estipuló que la expansión pre-establecida (proporcional) no es adecuada para la guía de onda estudiada.

\section{Análisis iterativo: búsqueda de mínimo.}

Observando que se obtuvieron ajustes aceptables con el modelo utilizado y que los valores de $d \omega / d p_{\text {num }}$ obtenidos difieren demasiado con aquellos medidos experimentalmente, surgió la siguiente pregunta: ¿Existe algún conjunto de parámetros de ajuste $\left(a, b, \alpha\right.$ y $\left.\beta, e_{i}, f_{i}\right)$ mediante los cuales se obtengan valores de $d \omega / d p_{\text {num }}$ aproximados a los de $\mathrm{d} \omega / \mathrm{dp}_{\mathrm{exp}}$ ? 
Esta formulación se consideró un criterio aceptable para obtener mayor información de la expansión producida en la interacción. Por lo tanto, se realizó un proceso iterativo que minimiza el máximo de la diferencia entre los valores de $\mathrm{d} \omega / \mathrm{dp} p_{\text {num }}$ obtenidos de sucesivos ajustes y los de d $\omega / \mathrm{dp}_{\mathrm{exp}}$. Para esto se utilizó la función de MATLAB "fminsearch.m" que minimiza algoritmos. Como esta función requiere definir valores iniciales para los parámetros a evaluar, se realizó primeramente un estudio global (etapa II) que se presenta a continuación.

\section{Etapa II: Variación de parámetros}

Para obtener una visión global del comportamiento del ajuste del modelo a las mediciones $\mu$-Raman, se realizaron ajustes de dicho modelo para un amplio rango de valores de sus parámetros ( $a, b$ y $\beta / \alpha$ ) y se comparó la desigualdad en la Ecuación 61 entre los valores de $d \omega / d p_{\text {exp }}$ y los de $d \omega / d p_{\text {num }}$ obtenidos para cada conjunto de parámetros. Los parámetros de ajuste son los valores de $e_{i}$ y $f_{i}$ asociados a cada fonón.

\section{Parámetros de expansión proporcionales.}

Manteniendo, en el modelo numérico, una geometría de elipse fija ( $a$ y $b$ ), y utilizando distintos valores de $\alpha$ y $\beta$ para ajustar los datos experimentales se puede observar lo siguiente: Aplicando una expansión / y otra expansión $m$, cuyos parámetros de expansión guarden una determinada proporción. Es decir que $\alpha_{\mathrm{m}}=K \alpha_{1}$ y $\beta_{\mathrm{m}}=K \beta_{\text {I. }}$. Donde $K$ es una constante de proporcionalidad. Si se ajustan estas dos expansiones a los datos experimentales, estos ajustes dan valores proporcionales en un factor $(1 / K)$ para $e, f y$ $d \omega / d p$, y un mismo error cuadrático medio. Es decir que; $d \omega / d p_{m}=(1 / K)^{*} d \omega / d p_{\text {; }}$ $e_{m}=(1 / K)^{*} e_{l}$ y $f_{m}=(1 / K)^{\star} f_{l}$, para cada fonón. Entonces, se puede afirmar que: 'expansiones proporcionales generan parámetros de ajuste (e y f) proporcionales y el mismo valor de $E C M$ '. Teniendo en cuenta esto, carece de sentido evaluar el ajuste para dos casos en que $\beta / \alpha$ tengan el mismo valor, por consiguiente se definió este cociente $(\beta / \alpha)$ como parámetro.

Se evaluaron como sigue los parámetros del modelo:

$a=\{0.5 ; 1 ; 1.5 ; 2 ; 2.5 ; 3 ; 3.5\}$

$b=\{5 ; 7 ; 8 ; 9 ; 11 ; 13\}$

$\beta / \alpha=\{0 ; 0.001 ; 0.01 ; 1 ; 10 ; 100 ; 1000\}$

Se ajustaron los corrimientos Raman en la trayectoria b'b", utilizando todas las combinaciones posibles entre los valores presentados. Los parámetros de ajuste fueron e y f. Como resultado se obtuvieron 294 (7x6x7) conjuntos de valores diferentes para los parámetros presentados en la Tabla vi: $e, f, d \omega / d p_{\text {num }}$ y ECM para cada fonón.

Se evaluó la siguiente condición de desigualdades en los 294 conjuntos de valores. 
$\left(\mathrm{d} \omega / \mathrm{dp} p_{2}>\mathrm{d} \omega / \mathrm{dp} p_{1}\right) \&\left(\mathrm{~d} \omega / \mathrm{dp} p_{2}>\mathrm{d} \omega / \mathrm{dp} p_{4}\right) \&\left(\mathrm{~d} \omega / \mathrm{dp} p_{3}>\mathrm{d} \omega / \mathrm{dp} p_{1}\right) \&\left(\mathrm{~d} \omega / \mathrm{dp} p_{3}>\mathrm{d} \omega / \mathrm{dp} p_{4}\right)$

La misma, es necesaria pero no suficiente para que concuerden los valores experimentales de $\mathrm{d} \omega / \mathrm{dp}_{\mathrm{exp}}$ con los valores obtenidos del ajuste numérico. Entonces, se pueden utilizar aquellos conjuntos de valores que cumplan esta condición, para conocer el comportamiento del sistema, acotar el espacio de parámetros y definir parámetros iniciales para el proceso iterativo.

La desigualdad presentada (Ecuación 71) es la más relevante debido a que los valores de $d \omega / d p_{1}$ y $d \omega / d p_{4}$ son muy cercanos, al igual que $d \omega / d p_{3}$ y $d \omega / d p_{2}$. Entonces, formular una desigualdad entre los elementos de estos pares, implica una exigencia que el ajuste no llega a diferenciar debido a las incertezas en las mediciones.

Luego del análisis de los 294 conjuntos, se encontró que aproximadamente la mitad de combinaciones cumplen la desigualdad propuesta y corresponden a la combinación de los siguientes parámetros:

$\mathbf{a}=\{0.5 ; 1 ; 2.5 ; 3 ; 3.5\}$

$\mathbf{b}=\{5,7,8,9,11,13\}$

$\beta / \alpha=\{0 ; 0.001 ; 0.01\}$

El cumplimiento de la desigualdad presentada en la Ecuación 71 para el parámetro a tiene un rango amplio que va desde 0.5 a $3.5 \mu \mathrm{m}$. Lo mismo ocurre con el parámetro $b$, este va desde 5 a 13. Mientras que para el parámetro $\beta / \alpha$ el rango se extiende a los valores de $\alpha$ y $\beta$ que cumplan la condición de $\alpha>\beta$. Creemos que esta última es la tendencia más importante obtenida en esta etapa, porque implicaría que la expansión es principalmente en dirección horizontal (coincidente con el eje cristalino $\mathrm{z}_{\mathrm{c}}$ ).

Etapa III: Búsqueda de mínimo

Para el proceso de búsqueda de mínimo error se definen los siguientes parámetros iniciales:

$$
a=2 \mu \mathrm{m} ; b=8 \mu \mathrm{m} ; \alpha=0.02 ; \beta=0.0
$$

Para los parámetros $a$ y $b$, se eligieron valores intermedios de los intervalos analizados en la etapa II. Por otro lado, se definió $\beta=0$ por simplicidad. Entonces, utilizando la función de MATLAB "fminsearch.m" que minimiza funciones, se minimizó la siguiente expresión:

$$
\left.D=\max \left(\left(\Delta d \omega_{1} / d p\right)^{2},\left(\Delta d \omega_{2} / d p\right)^{2},\left(\Delta d \omega_{3} / d p\right)^{2},\left(\Delta d \omega_{4} / d p\right)^{2}\right)\right]
$$


Donde $\Delta d \omega_{i} / d p$ es la diferencia entre el coeficiente de presión experimental y numérico para cada fonón $\mathrm{A}_{1}(\mathrm{TO})_{\mathrm{i}}: \Delta d \omega_{i} / d p=d \omega_{i} / d p_{\exp }-d \omega_{i} / d p_{\text {num }}$. Por lo tanto, $D$ representa la máxima diferencia (elevada al cuadrado) entre los valores experimentales y numéricos de $d \omega / d p$. De este proceso, se obtuvo: $\boldsymbol{a}=2.2, \boldsymbol{b}=6.2, \boldsymbol{\alpha}=0.022 ; \boldsymbol{\beta}=-0.001$.

\begin{tabular}{|c|c|c|c|c|c|}
\hline Fonón & $e$ & $f$ & $\begin{array}{c}\mathrm{d} \omega / \mathrm{dp} \mathrm{num}_{\mathrm{m}} \\
{\left[\mathrm{cm}^{-1} / \mathrm{Gpa}\right]}\end{array}$ & $\begin{array}{c}\mathrm{d} \omega / \mathrm{dp} \text { exp } \\
{\left[\mathrm{cm}^{-1} / \mathrm{Gpa}\right]}\end{array}$ & $\mathrm{ECM}$ \\
\hline $\mathrm{A}_{1} \mathrm{TO}_{1}$ & $-278,78$ & 80,146 & 1.6 & 0.7 & 0.09 \\
\hline $\mathrm{A}_{1} \mathrm{TO}_{2}$ & $-672,57$ & $-465,68$ & 5.3 & 5.5 & 0.1 \\
\hline $\mathrm{A}_{1} \mathrm{TO}_{3}$ & $-613,41$ & $-298,62$ & 4.6 & 6.6 & 0.3 \\
\hline $\mathrm{A}_{1} \mathrm{TO}_{4}$ & $-146,56$ & 185,32 & 0.2 & 1.1 & 0.07 \\
\hline
\end{tabular}

Tabla viii. Resultado del ajuste iterativo unidimensional.
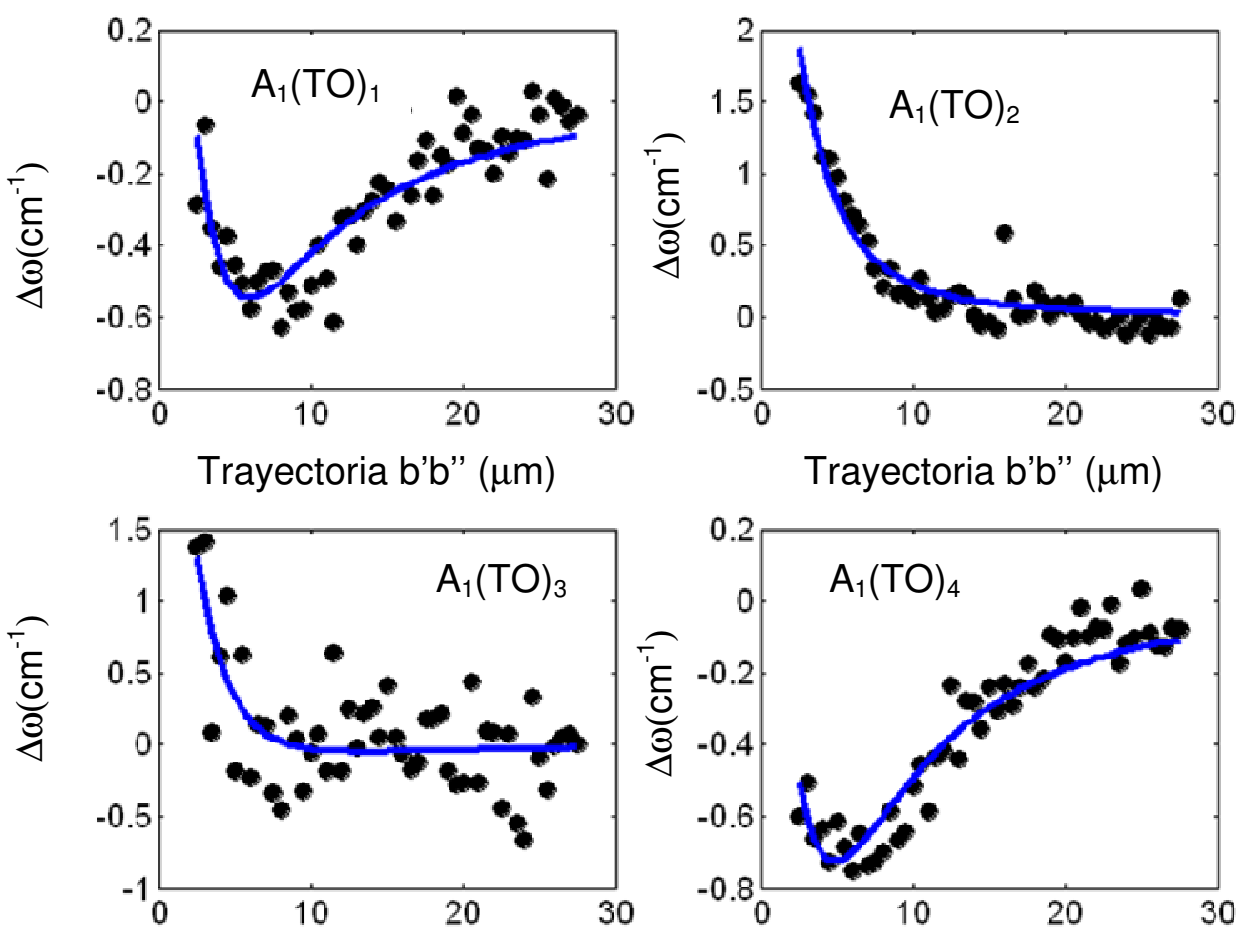

Trayectoria b'b" $(\mu \mathrm{m})$

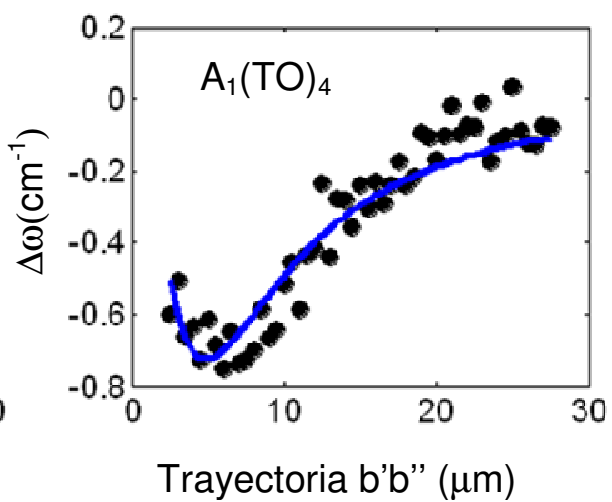

Figura 52. Resultado de ajuste iterativo de los fonones $A_{1}(T O)_{i}$ a lo largo de la trayectoria b'b" $(\mu \mathrm{m})$. 
Se puede observar en la Tabla viii, que los valores de $d \omega / d p_{\text {num }}$ obtenidos se acercan más a los valores de $d \omega / d p_{\exp }$ que aquellos obtenidos en la etapa I. En la Figura 52 se puede observar que el modelo ajusta mejor a los corrimientos experimentales Raman, que el primer ajuste realizado (etapa I), principalmente para los fonones $A_{1} T O_{1}$ y $A_{1} T O_{4}$. A su vez, todos los ajusten tienen un ECM menor a 0.1 , a excepción del $A_{1}(T O)_{3}$, que posee un ECM de 0.3. Este fonón tiene una dispersión considerablemente mayor en los datos experimentales, como se ve en la Figura 52. Los valores de e y $f$ obtenidos en este ajuste iterativo son, en general, negativos y poseen menor magnitud que los obtenidos del primer ajuste (etapa I).

- Resumen de análisis unidimensional

En este análisis, se estudiaron diversos ajustes unidimensionales entre el modelo numérico de elementos finitos propuesto y la medida del corrimiento $\mu$-Raman de los cuatro fonones Raman $\mathrm{A}_{1} \mathrm{TO}_{i}$ del niobato de litio. De estos ajustes, se considera significativo que el modelo ajustó de forma adecuada, para los parámetros propuestos en la trayectoria b'b" y que, en principio, la expansión sería en la dirección horizontal (eje cristalino $\mathrm{z}_{\mathrm{c}}$ ).

A su vez, se consideró necesario realizar un estudio bidimensional de las guías de onda para contrastar los resultados obtenidos en este análisis y obtener mayor información del campo de deformaciones e índice de refracción.

\subsubsection{Análisis bidimensional de guía de onda (modificación B)}

En esta subsección, se presenta un estudio bidimensional de la zona de guiado de luz lindante a la modificación $B$. En este análisis, se ajustó de forma iterativa, el campo de corrimientos Raman al modelo numérico previamente desarrollado, para obtener valores óptimos de los parámetros del modelo. También se utilizó la intensidad guiada medida experimentalmente para caracterizar la expansión producida en la interacción, comparándola con aquella obtenida numéricamente. A su vez, se utiliza el modelo numérico para obtener las constantes de deformación potencial $(e$ y $f$ ).

Se realizó un mapeo $\mu$-Raman bidimensional sobre la modificación estructural B y una medición de modos guiados en las zonas lindantes a la misma. Las mediciones de los mapas $\mu$-Raman fueron realizadas por el autor de este trabajo en la Universidad Autónoma de Madrid (España), bajo la tutela del Dr. D. Jaque. La medición de la intensidad guiada también fue realizada por el mismo en el ClOp. En la Figura 53, se presentan: (a) una imagen óptica de la zona analizada, (b) la imagen de modos acoplados con láser rojo $(650 \mathrm{~nm})$ en las guías ubicadas a ambos lados de la modificación estructural, (c) la medida de un espectro Raman en un punto arbitrario de la 
región y los mapas de corrimientos espectrales de los fonones (d) $A_{1}(T O)_{1}$, (e) $A_{1}(T O)_{2}$ y (f) $A_{1}(T O)_{4}$ en la zona de mapeo. No se ha detectado señal para el corrimiento del fonón $\mathrm{A}_{1} \mathrm{TO}_{3}{ }^{7}$.
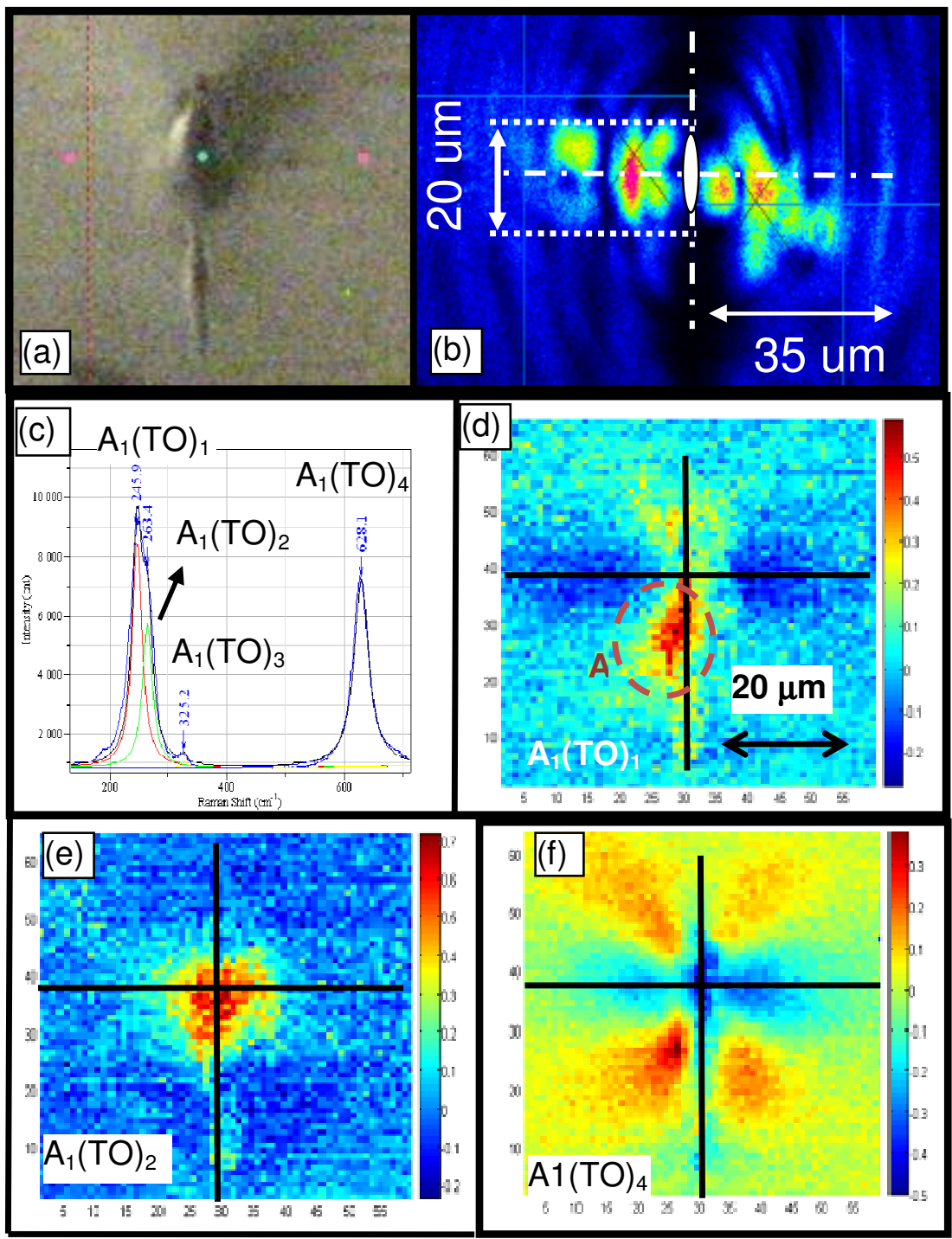

Figura 53. (a) Imagen óptica de modificación estructural analizada. (b) Principales modos guiados en ambos lados de la modificación (650 nm). (c) Espectro Raman medido en un punto genérico y ajuste de funciones lorentzianas. Mapa de corrimiento espectral $\Delta \omega$ : (d) Fonón $\mathrm{A}_{1} \mathrm{TO}_{1}$, (e) Fonón $\mathrm{A}_{1} \mathrm{TO}_{2}$, (f) Fonón $\mathrm{A}_{1} \mathrm{TO}_{4}$.

Se puede observar que la distribuciones de intensidad guiada que se presentan en la Figura 53 son asimétricas respecto de la modificación generada por el láser de fs: a la derecha se observan modos que están menos confinados en la dirección vertical. En

\footnotetext{
${ }^{7}$ Para el fonón $\mathrm{A}_{1} \mathrm{TO}_{3}$ no se obtuvo señal al medir el corrimiento en número de onda, esto se debe a que la relación señal ruido de este fonón es cosiderablemente menor a los otros fonones en las medidas analizadas (Ver Figura 53(c)).
} 
cambio, a la izquierda de la modificación, los modos guiados tienen un mayor confinamiento en la dirección vertical y tienen una leve asimetría con respecto al eje horizontal que pasa por el centro de la modificación principal.

Por otro lado, de las medidas del corrimiento de los fonones (Figura 53 (d), (e) y (f)) se observa que la que tiene mejor señal es la del fonón $A_{1}(T O)_{4}$. Este fonón tiene un corrimiento de una magnitud máxima de $0.3 \mathrm{~cm}^{-1}$ aproximadamente. Este corrimiento es menor que aquel medido en la modificación A. Esto se debe a que en la modificación A, la energía enfocada se concentra en una sola región porque no se produce filamentación, mientras que en la modificación B la energía enfocada se divide entre la modificación principal y la secundaria producida por el fenómeno de filamentación.

También se puede ver en la Figura 53(f), que el comportamiento de los modos guiados está en concordancia con los lóbulos negativos (azules), corrimientos del fonón $A_{1}(T O)_{4}$ hacia menores números de onda. El lóbulo azul de la izquierda es de menor área que el de la derecha. En la región del primero, la intensidad guiada se encuentra más confinada en la dirección vertical, qué en la región del segundo.

El mapa de corrimiento $(\Delta \omega)$ del fonón $A_{1}(T O)_{1}$, aunque posee una menor relación señal ruido, tiene un comportamiento similar al del fonón $\mathrm{A}_{1}(\mathrm{TO})_{4}$ y posee una suerte de singularidad en la zona cercana a la modificación estructural, que se encierra con la curva de a trazos A (Figura 53(d)) .

En consecuencia, el mapeo bidimensional revela que el fonón $\left(A_{1} T O\right)_{4}$ observa una importante correlación con los modos guiados, y en consecuencia, con el campo de índice de refracción resultante de la interacción.

De todas formas, se puede inferir de la Figura 53, que los modos guiados (ó distribuciones de intensidad) son más sensibles que el mapa de corrimiento $\mu$-Raman a las variaciones de índice de refracción.

- Comparación con parámetros calculados en estudio unidimensional.

Cabe recordar, que el modelo numérico propuesto para estudiar las guías de onda, consiste en un cuarto de elipse (con radios $a$ y $b$ ) que se expande con parámetros $\alpha$ y $\beta$. Durante el estudio unidimensional, se optimizaron los parámetros $(a, b, \alpha$ y $\beta)$ del modelo numérico propuesto, teniendo en cuenta los coeficientes de presión hidrostática $d \omega / d p_{\mathrm{i}}$ obtenidos experimentalmente y las medidas de corrimiento Raman en la trayectoria b'b" en modificación A. Como resultado, se obtuvieron valores tentativos de los cuatro parámetros mencionados y de las constantes de deformación potencial (e y $f$ ). Para analizar la validez del modelo numérico propuesto en el dominio bidimensional, se calculó el mapa bidimensional de corrimiento Raman para los cuatro fonones $A_{1}(T O)$ utilizando los parámetros obtenidos en el estudio unidimensional. Estos mapas obtenidos numéricamente se comparan con los mapas bidimensionales obtenidos experimentalmente en la Figura 54. 


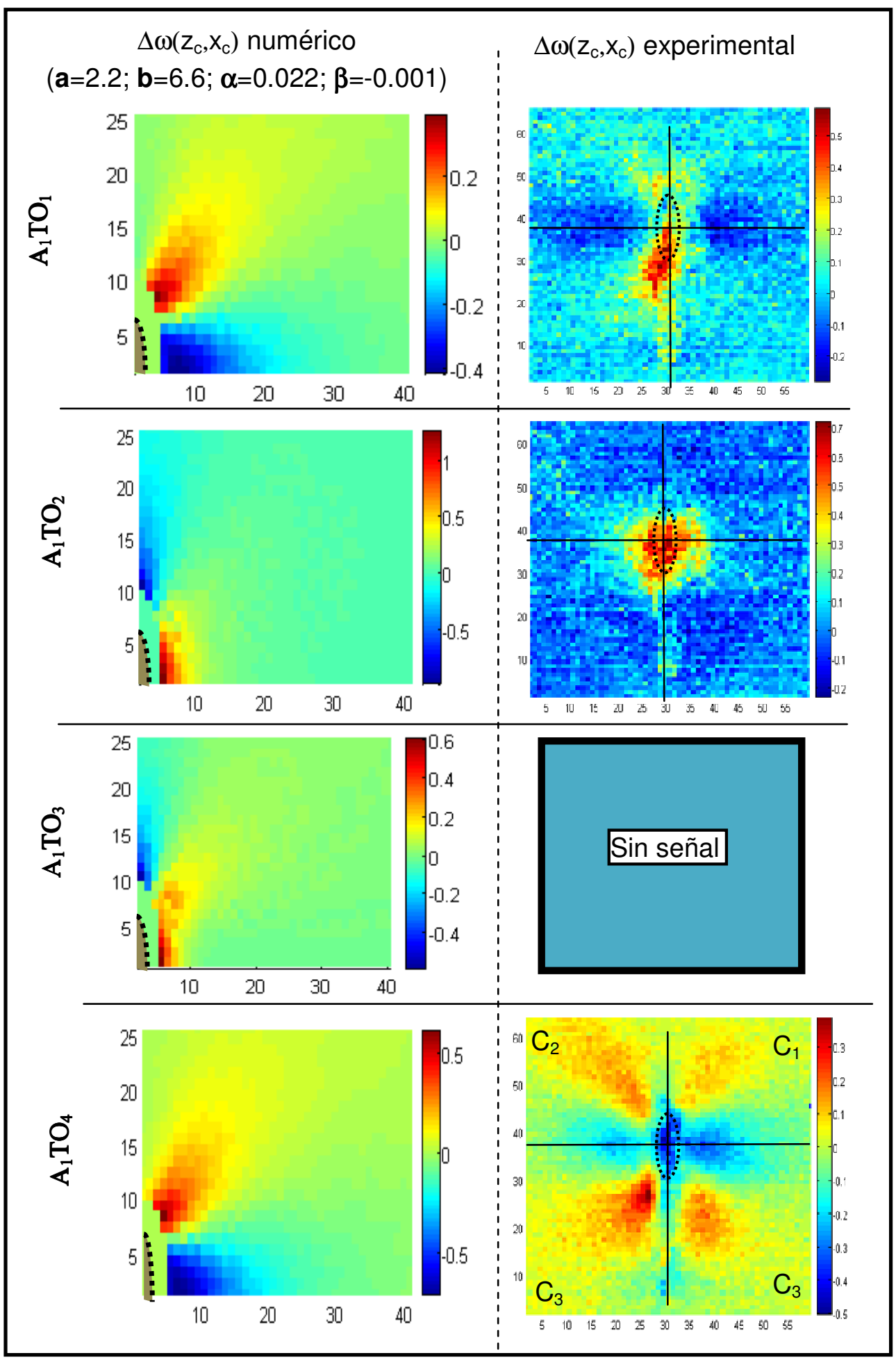

Figura 54. Resultados del modelo numérico con parámetros obtenidos del análisis unidimensional (Tabla viii) y medidas experimentales. 
Se debe tener en cuenta que los mapas a la izquierda, en la Figura 54, representan un cuarto de dominio. Mientras que las medidas experimentales a la derecha, representan el mapa experimental para el dominio completo. Además, no se presenta la zona cercana a elipse obtenida en el modelo numérico porque el campo de deformaciones toma valores extremadamente altos. Por consiguiente, el campo de corrimientos Raman numérico también y esto dificulta la comparación con los mapas experimentales. Como determinaron A.Ródenas y colaboradores [RO09], en la zona cercana a la modificación estructural también se producen cambios estructurales en el material, aunque en menor grado que la zona focal. Con lo cual, la aproximación elástica del fenómeno no resulta adecuada para la misma.

Comparando los resultados numéricos con los experimentales (Figura 54), se puede concluir que el mapa numérico correspondiente al fonón $\mathrm{A}_{1}(\mathrm{TO})_{4}$ es el que mejor ajusta a los corrimientos espectrales medidos experimentalmente: el cuarto de dominio posee dos lóbulos en forma similar a la medida experimental. El mapa numérico del fonón $A_{1}(T O)_{1}$ también tiene un comportamiento acorde a la medida experimental, aunque en menor medida que el fonón $\mathrm{A}_{1}(\mathrm{TO})_{4}$, ya que los lóbulos positivos no se logran distinguir claramente en la medida experimental. Por otro lado, el mapa experimental del fonón $\mathrm{A}_{1}(\mathrm{TO})_{2}$ observa un comportamiento cualitativamente similar al predicho por el modelo, pero en menor grado que los anteriores y con menor relación señal-ruido: el mapa experimental tiene menor grado de simetría y en la parte superior de la elipse no se distingue claramente el lóbulo negativo observado en el mapa numérico.

Debido a que el modelo implementado tuvo, cualitativamente, buena concordancia para el dominio bidimensional, en particular para los corrimientos medidos para el fonón $\mathrm{A}_{1}(\mathrm{TO})_{4}$. En las secciones subsiguientes, se calcularán nuevamente los parámetros óptimos del modelo ( $a, b, \alpha$ y $\beta$ ) considerando el mapa bidimensional experimental del fonón $A_{1}(T O)_{4}$ e incorporando el cálculo numérico de modos guiados. Una vez definido el campo de deformaciones, el mismo se ajustará a los demás fonones $A_{1}(T O)$ para obtener valores de las constantes de deformación potencial e y $f$.

- Indicadores de ajustes.

Para realizar la optimización propuesta, es necesario definir los indicadores que serán utilizados para comparar los distintos ajustes a las medidas experimentales.

Salvo indicación contraria, los parámetros de ajuste son las constantes de deformación potencial e y $f$.

ETC: Es el Error Total Cuadrático entre el ajuste realizado y la medida experimental. Se define como la sumatoria de los cuadrados de la diferencia entre el ajuste y los datos experimentales, para los distintos puntos (Ecuación 73). Se asume que indica la bondad 
de un determinado ajuste. Los ajustes bidimensionales se realizaron minimizando el ETC mediante la función de Matlab fminsearch.m.

$E T C=\operatorname{Sum}\left[\left(\Delta \omega_{\text {exp }}-\Delta \omega_{\text {num }}\right)^{2}\right]$

$\boldsymbol{f}_{i} / \boldsymbol{e}_{i}$ : Este cociente define, en un determinado ajuste, cuál de las deformaciones tiene mayor peso: si $\varepsilon_{x c}$ ó $\varepsilon_{z c}$. Como ya se mencionó, los corrimientos experimentales de número de onda $\Delta \omega$ se asumen dados por: $e_{i} \varepsilon_{x c}+f_{i} \varepsilon_{z c}$. En donde e y $f$ son utilizados como parámetros de ajuste y representan las Constantes de Deformación Potencial, a priori desconocidas. El cociente $f_{i} / e_{i}$ es útil para definir si dos ajustes con igual o muy cercano valor de ETC son "cuasi-equivalentes" ú "opuestos". Además utilizando este cociente nos independizamos de las magnitudes de $f$ y $e$, que a priori no son conocidas.

- Promedio de cuadrantes.

Debido a que el modelo numérico es simétrico, y la medida experimental tiene un importante grado de simetría (Figura 54), se realizó un promedio entre los cuatro cuadrantes $\left(\mathrm{C}_{1}, \mathrm{C}_{2}, \mathrm{C}_{3}\right.$ y $\left.\mathrm{C}_{4}\right)$. El mapa resultante se muestra en la Figura 55. El mismo se utilizará a continuación, para optimizar los parámetros del modelo numérico $(a, b$ y $\beta / \alpha)$, en una primera aproximación. Para realizar dicho proceso de optimización, se quitaron los datos que están en las cercanías de la modificación generada por interacción directa con el láser (dentro de una elipse de radios $1.4^{\star} a$ y $1.4^{\star} b$ ), ya que, como se mencionó en la sub-sección anterior, en esta zona se producen considerables plasticidades/modificaciones estructurales del material [RO09] y no sería válida la aproximación de elasticidad lineal.

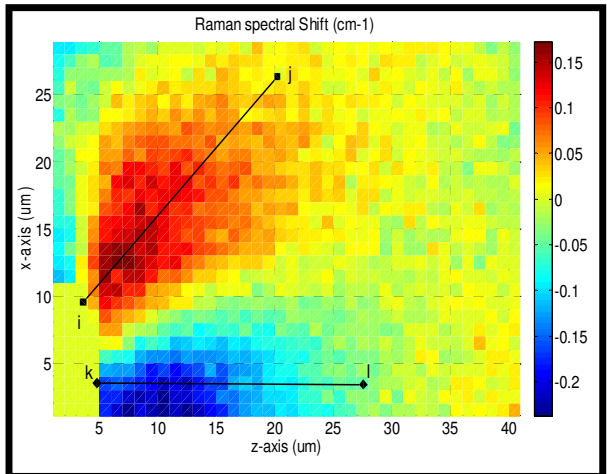

Figura 55. Mapa de corrimiento Raman de fonón $\mathrm{A}_{1}(\mathrm{TO})_{4}$ promediado.

- Optimización de parámetros

La optimización de los parámetros del modelo numérico $(a, b, \alpha$ y $\beta$ ) para el mapa bidimensional experimental del fonón $\mathrm{A}_{1}(\mathrm{TO})_{4}$ promediado (Figura 55), se realizó en varias etapas. En la primera, se fijaron los valores de $\alpha$ y $\beta$ iguales a 0.01 , para 
determinar los valores de a y $b$ (radios de la elipse) óptimos. En la segunda etapa, se fijaron los valores de $a$ y $b$ obtenidos como óptimos, para obtener los rangos óptimos de $\beta / \alpha$. Para interpretar la variación del ETC en función de $\beta / \alpha$ se utilizó el cociente f/e. Luego, se realizó el cálculo de la distribución de intensidad guiada para numerosas combinaciones de $\alpha$ y $\beta$ utilizando los parámetros geométricos $\left(\begin{array}{lll}a & \text { y } & b\end{array}\right)$ definidos inicialmente como óptimos.

\section{Estimación de a y b óptimos}

A continuación se describe el procedimiento llevado a cabo para determinar los valores óptimos de $a$ y $b$.

Se calcularon, utilizando el código script2.m (presentado en Anexo B), los valores de ETC para las distintas combinaciones de parámetros $a$ y $b$ (denominados parámetros geométricos) considerando una expansión fija dada por $\alpha=0.01$ y $\beta=0.01$. Los rangos considerados para los parámetros a y $b$ son los siguientes: desde 0.1 a $3 \mu \mathrm{m}$ para el primero y, desde 6 a $12 \mu \mathrm{m}$ para el segundo. Para obtener el ETC, se ajustó el mapa numérico al mapa experimental para cada combinación de los valores de a y b. Esto se realizó utilizando e y $f$ como parámetros de ajuste y la función de MATLAB fminsearch.m.

El resultado se representa con curvas de nivel en la Figura 56. En la misma, se aprecia un mínimo absoluto para los valores de $a=1.7$ y $b=8.5$. Esto se remarca en dicha figura con línea de a trazos.

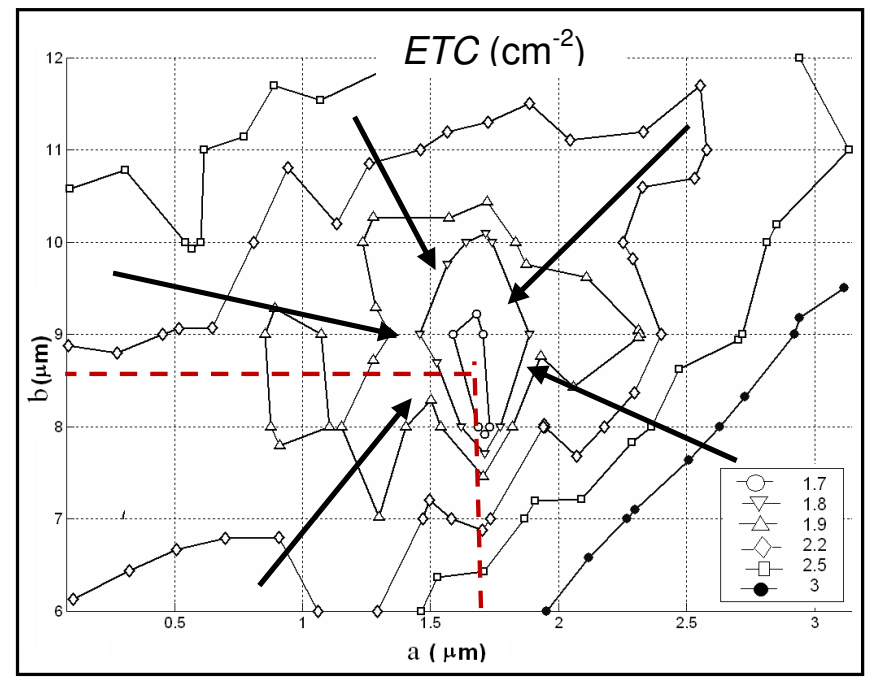

Figura 56. Suma de error cuadrático ETC como función de las dimensiones a y $b$ de la elipse.

\section{Estimación de $\beta / \alpha$ óptimos}

Una vez encontrados los parámetros geométricos óptimos ( $a$ y $b$ ), el paso siguiente fue evaluar el Error Total Cuadrático (ETC) para distintas combinaciones de $\beta / \alpha$. Como ya se 
mencionó, esta relación adimensional indica en qué dirección es más importante la expansión. Por lo tanto, considerando sólo expansiones positivas (se asume que son predominantes los desplazamientos hacia el exterior del elipse, con la consecuente disminución de densidad en la región de interacción directa con láser de fs [BUR07, BURHA07]), este cociente puede tomar valores desde cero (expansión horizontal "pura") hasta infinito (expansión vertical "pura") en el modelo numérico. Por lo tanto, este cociente se evaluó desde $10^{-5}$ hasta $10^{5}$. En la Figura 57 (a), se muestra el indicador ETC en función de $\beta / \alpha$. En la misma, se observa que el ETC es mínimo en dos zonas del rango analizado. Una de estas, ocurre para $\alpha>>\beta$ y por lo tanto esta es una expansión horizontal y se denominará "caso l". La otra condición ocurre para $\beta>>\alpha$, siendo ésta una expansión vertical y se denominará "caso II". En el caso I, el ETC es levemente menor que en el caso II. Esta diferencia se consideró demasiado pequeña $\left(<0.2 \mathrm{~cm}^{-1}\right)$ para definir que el primer caso es más adecuado que el segundo. También se puede observar en la misma Figura, que el ETC es prácticamente constante para ambos casos. Como consecuencia, a priori, cualquiera de estas opciones, desde el punto de vista del ajuste del mapa $\mu$-Raman, le dan al problema una solución óptima. Por lo tanto, el mapa del fonón $\mathrm{A}_{1}(\mathrm{TO})_{4}$ no permite definir la característica de expansión mecánica que ocurre si se desconocen los valores de e y $f$.

Simultáneamente, se evaluó el cociente f/e en función del cociente $\beta / \alpha$, este indica la importancia relativa entre las deformaciones $\varepsilon_{\mathrm{xc}}$ y $\varepsilon_{\mathrm{zc}}$ de un determinado ajuste. Esta dependencia se muestra en la Figura 57 (b). Se puede observar que, los cocientes f/e que minimizan el ETC son para $\beta / \alpha<<1$ y $\beta / \alpha>>1$. Estos casos, son sustancialmente distintos y el ETC es prácticamente constante en cada caso (en el primero tiene valores cercanos a 0.1 y en el segundo cercanos a 2.5). Esto implica que en el caso I se le da mayor "peso" a las deformaciones $\varepsilon_{z c}$, mientras que en el caso II esto ocurre con las deformaciones $\varepsilon_{\mathrm{xc}}$. 

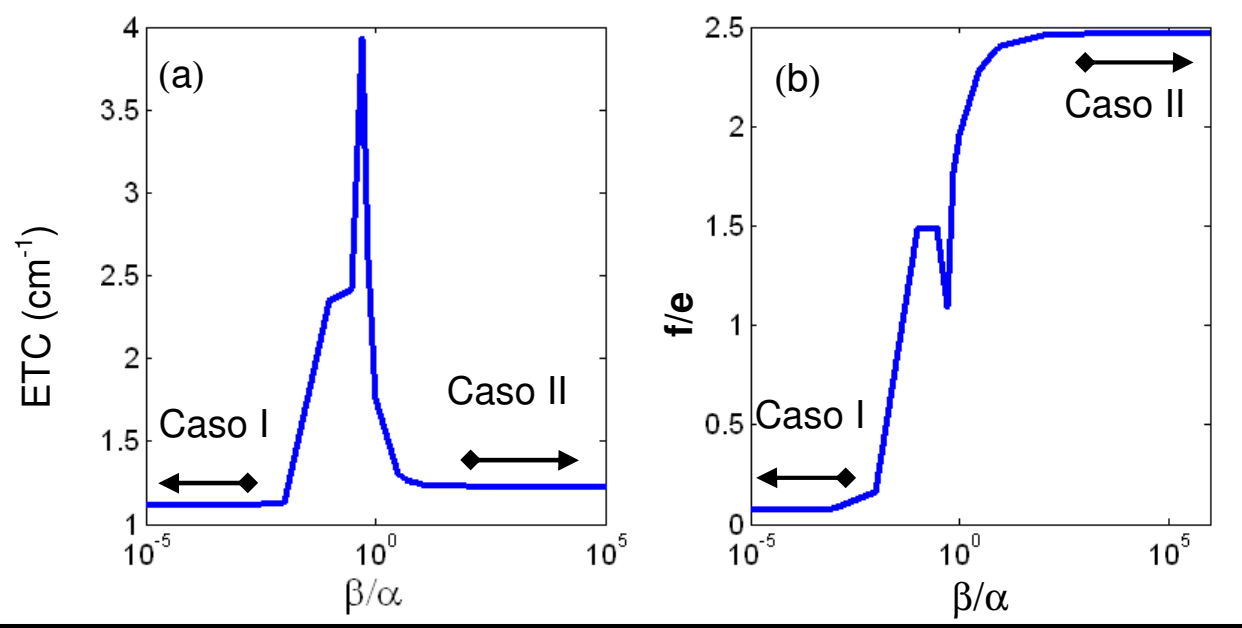

Figura 57.(a) Error Total (ETC) en función de $\beta / \alpha$ y (b) cociente entre constantes de deformación potencial f/e en función de $\beta / \alpha$.

Para discernir cuál de estas dos opciones (caso I ó caso II) ajusta mejor a la expansión que realmente ocurre en el material, se calcularon los modos guiados para los valores de $a$ y $b$ calculados como óptimos (1.7 y $8.5 \mu \mathrm{m}$, respectivamente) y para numerosas combinaciones de $\alpha$ y $\beta$. Los valores de ambos parámetros $(\alpha$ y $\beta$ ) utilizados, van desde 0 hasta 0.02 de a intervalos de 0.0025 . Como se describió en la Sección 4.4, para esto, primeramente se calcula el campo de deformaciones originado por una determinada expansión(codigo2.m), luego la variación de índice de refracción asociada (campoindtri2.m) y finalmente, la distribución de intensidades guiadas por este campo de índice de refracción(codigo3.m).

En la Figura 58, se muestran las distribuciones de intensidad obtenidas para las combinaciones de $\alpha$ y $\beta$ más representativas y la distribución de intensidad medida experimentalmente a la izquierda de la modificación B. Se pueden ver en la figura mencionada, que las características geométricas de las distintas distribuciones de intensidad para las distintas combinaciones de parámetros $\alpha$ y $\beta$ son considerablemente diferentes. A su vez, al aumentar las magnitudes de expansión ( $\alpha$ y $\beta$ ) se obtienen mayor cantidad de modos guiados, como es de esperar por el mayor incremento de índice.

El modo medido experimentalmente que se presenta en la Figura 58 , no es de una sola polarización. En cambio, para el modo calculado numéricamente, se utilizó la componente $x_{c}$ del índice de refracción, debido a que es la componente del índice de refracción que, según los trabajos en donde se propuso el modelo numérico, es la que permite guiar la luz [BUR07] en este tipo de guías. El aspecto vinculado a la polarización guiada se discutirá con mayor detalle en la Sección 5.4 , utilizando nuevas medidas experimentales y cálculos numéricos. 


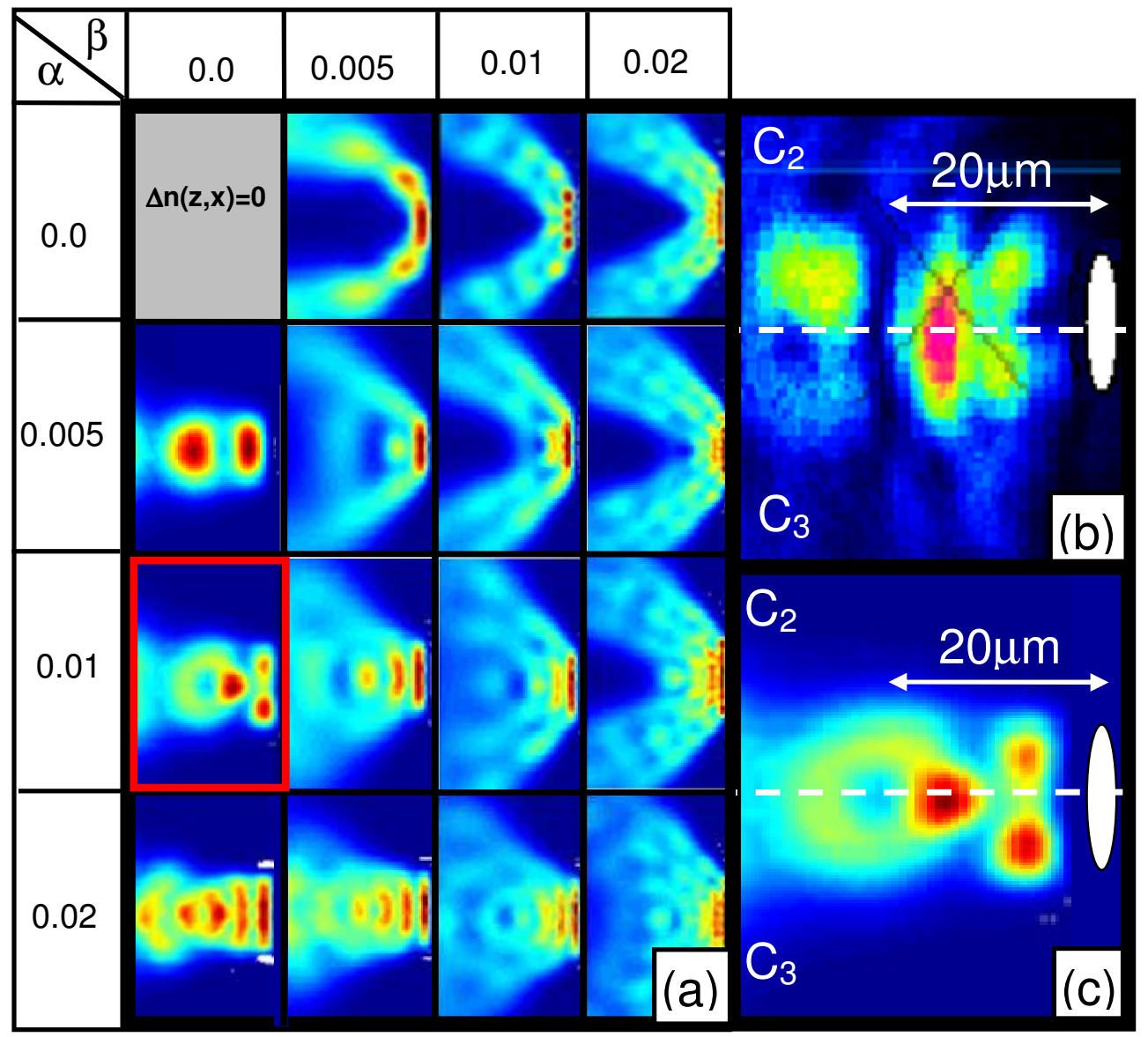

Figura 58. (a) Distribuciones de intensidad para distintos parámetros de expansión, (b) Distribución de intensidad experimental, (c) Ampliación de distribución de intensidad calculada para $\alpha=0.01$ y $\beta=0$.

En la Figura 58, se observa que la distribución de intensidad numérica para $\alpha=0.01$ y $\beta=0$ guardan una importante similitud con los modos guiados a la izquierda de la modificación. A su vez, esta expansión coincide con la obtenida en el análisis unidimensional.

Por otro lado, resulta más difícil establecer una correspondencia con los modos guiados a la derecha de la modificación. Se estima que estos se encuentran afectados por la modificación secundaria ubicada en la zona inferior y por simplicidad, no se presentan nuevamente. Por lo tanto, para la modificación analizada, el modelo utilizado tiene mejor concordancia con los cuadrantes $\mathrm{C}_{2}$ y $\mathrm{C}_{3}$ que con los cuadrantes $\mathrm{C}_{1}$ y $\mathrm{C}_{4}$ de la medida experimental (Ver Figura 53). A su vez, con respecto a la zona de guiado de luz, se puede observar en la figura anterior, que el cuadrante $\mathrm{C}_{2}$ (superior izquierdo) tiene mejor concordancia con el modelo numérico que el cuadrante $\mathrm{C}_{3}$ (inferior izquierdo). 


\section{Mapa de corrimiento espectral obtenido.}

En la Figura 59 (a) se presenta el mapa del corrimiento en número de onda medido experimentalmente (promedio de los cuatro cuadrantes) y en la Figura 59(b) se presenta el resultado del ajuste numérico de los corrimientos espectrales del fonón $A_{1}(T O)_{4}$ para los parámetros definidos como óptimos: $a=1.7 \mu \mathrm{m}, b=8 \mu \mathrm{m}, \alpha=0.01$ y $\beta=0$. Se puede observar una importante concordancia entre ambos mapas. Esto, permite corroborar la validez del modelo numérico para describir el campo de corrimiento espectral del fonón $\mathrm{A}_{1}(\mathrm{TO})_{4}$ en la zona de guiado.

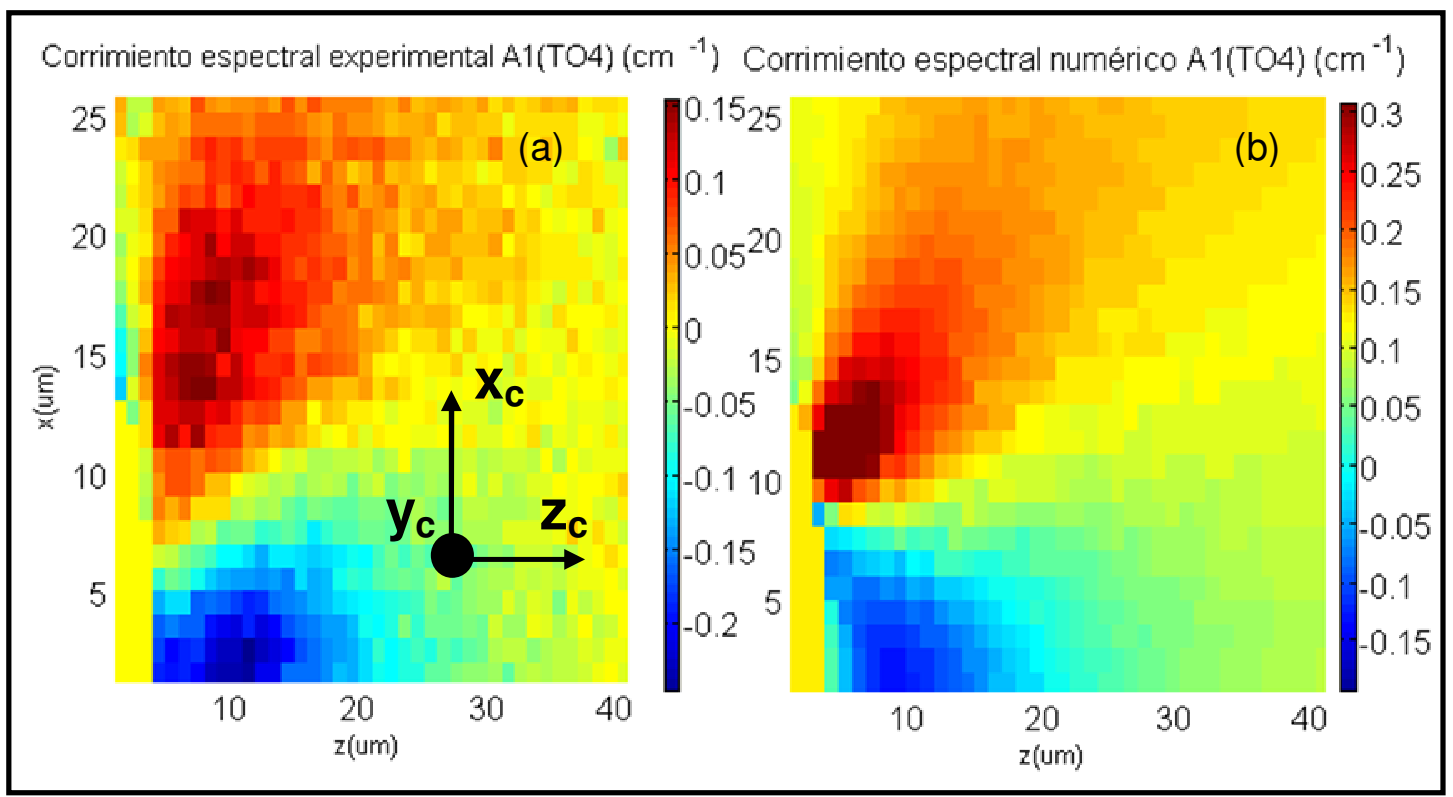

Figura 59. Mapa de corrimiento espectral del fonón $\mathrm{A}_{1}(\mathrm{TO})_{4}(\mathrm{a})$ medida experimental (promedio de cuadrantes), (b) ajuste del modelo numérico.

\subsubsection{Determinación de e y $f$}

Para obtener los valores absolutos aproximados de e y $f$, ya no resulta apropiado ajustar el modelo numérico al promedio de los cuatro cuadrantes experimentales del mapa de corrimiento Raman. Este ya no es un ajuste para comparar/optimizar valores de parámetros desconocidos. En este nuevo objetivo, se requiere mayor precisión porque se propone calcular valores absolutos de e y $f$. $Y$ los lóbulos correspondientes a los distintos cuadrantes, como se observa en la Figura 60, poseen distinta forma e intensidad. Por ejemplo, como ya se mencionó, el lóbulo negativo de la izquierda abarca un área menor que el de la derecha (ambos se encierran con una curva verde de línea de a trazos: A y $B$, respectivamente). Otra diferencia importante que se puede apreciar, es que el lóbulo positivo ubicado en el cuadrante $\mathrm{C}_{3}$, tiene magnitud considerablemente mayor (aproximadamente dos veces) que los correspondientes a los demás cuadrantes. Este lóbulo puede ser originado por una singularidad del material generada por la expansión, ya que se encuentra relativamente cerca de la modificación central y es 
considerablemente más agudo que los otros lóbulos positivos. Además, en esta zona, el fonón $A_{1}(T O)_{1}$ también posee una singularidad aún más notoria (encerrada por curva $A$ en Figura 53(d)).

De acuerdo a lo analizado en la sección anterior, una expansión horizontal $(\alpha=0.01, \beta=0)$ en el modelo numérico, se corresponde en mayor medida con la intensidad guiada a la izquierda de la modificación (en los cuadrantes $\mathrm{C}_{2}$ y $\mathrm{C}_{3}$ ). Debido a que esta distribución de intensidad guiada se corresponde con el lóbulo azul (curva A en Figura 60), la misma no presenta un grado importante de asimetría respecto del eje de simetría horizontal. La intensidad guiada experimentalmente en el cuadrante $\mathrm{C}_{2}$ guarda mayor similitud con la distribución de intensidad guiada obtenida numéricamente. A su vez, entre los lóbulos positivos que se encuentran en los cuadrantes $\mathrm{C}_{2}$ y $\mathrm{C}_{3}$, sí se observa una diferencia considerable en sus magnitudes. Esta diferencia no afecta directamente a los modos guiados por encontrarse fuera de la región de guiado, pero sí afecta al cálculo de las constantes de deformación potencial $(e$ y $f$ ). Se asumió que la región inferior de la modificación (cuadrante $\mathrm{C}_{3}$ ) está afectada directamente por la modificación secundaria o filamentación (curva D en Figura 60) y por consiguiente no era apropiado ajustar está región al modelo numérico para obtener valores de e y $f$. Por estos motivos, se asumió que el cuadrante $\mathrm{C}_{2}$ era el más apropiado para obtener valores absolutos de e y $f$.

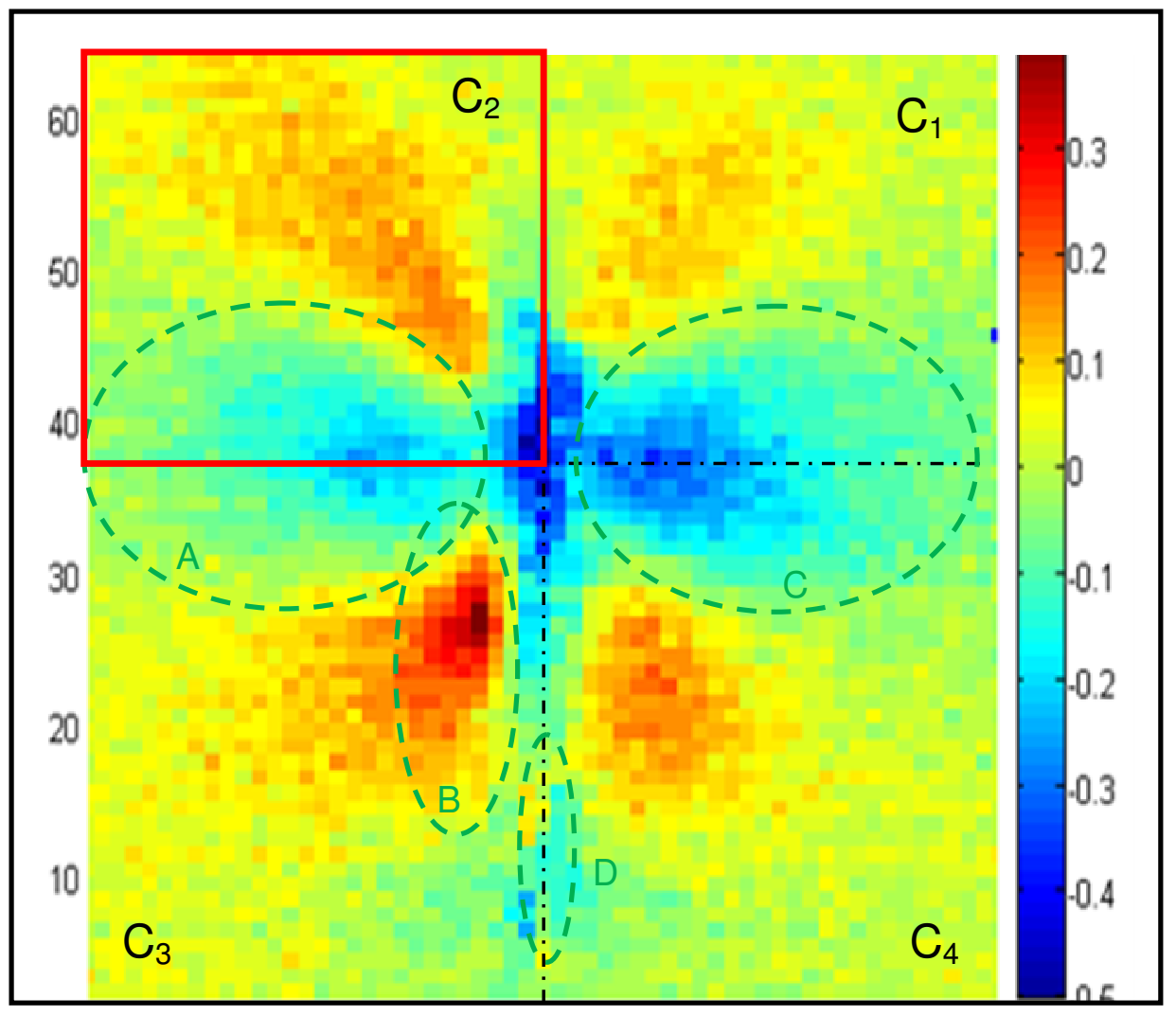

Figura 60. Medida experimental de corrimiento Raman en modificación B. 
Por lo tanto, se volvieron a calcular los valores óptimos de a y $b$, pero esta vez utilizando la expansión definida como óptima en la sección anterior $(\alpha=0.01$ y $\beta=0.00)$ y los datos experimentales del cuadrante $\mathrm{C}_{2}$. Por lo tanto, se calculó el Error Total Cuadrático para distintos valores de $a$ (desde 0.1 a $3 \mu \mathrm{m}$ ) y $b$ (desde 6 a $12 \mu \mathrm{m}$ ) para el cuadrante $\mathrm{C}_{2}$ y se obtuvo la superficie presentada en la Figura 61. En la misma, se puede ver que los valores de $a$ y $b$ óptimos para este caso son: $a=3 \mu \mathrm{m}$ y $b=7.5 \mu \mathrm{m}$ (los que no difieren demasiado de $a=1.7$ y $b=8.5)$. Utilizando estos nuevos valores de $a$ y $b$, y la expansión definida como óptima para este cuadrante, $\alpha=0.01$ y $\beta=0.0$, se obtuvo nuevamente la distribución de intensidad guiada usando el modelo numérico. Esto, se realizó para verificar que los parámetros obtenidos $(a=3, b=7.5, \alpha=0.01, \beta=0)$ ajusten tanto al mapa de corrimiento Raman (minimización de ETC) como así también, a la distribución de intensidad guiada.

Se puede ver en la Figura 61, que esta nueva distribución de intensidades (Figura 61 (b)) resultante también guarda una importante similitud con la medida experimental (Figura 61 (c)). Una vez fijados estos parámetros, se ajustó el campo de deformaciones resultante a la medida de corrimiento Raman experimental del cuadrante $C_{2}$, obteniéndose $f_{4} \approx-550$, $e_{4} \approx-160$ y $\mathrm{d} \omega / \mathrm{dp}_{\mathrm{num}} \approx 4 \mathrm{~cm}^{-1} / \mathrm{GPa}$.

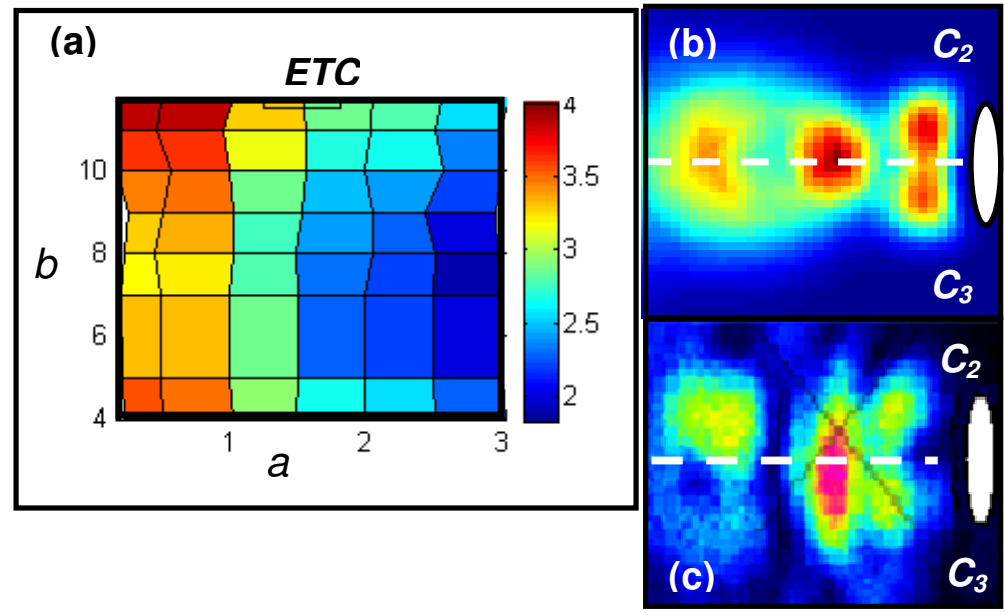

Figura 61. (a)ETC vs a vs $b$ para el cuadrante $\mathrm{C}_{2}$ (b)Intensidad guiada calculada numéricamente $(a=3, b=7.5, \alpha=0.01, \beta=0)$, (c) Intensidad guiada experimentalemente a la izquierda de la moficiación $B$.

Para determinar la magnitud de expansión $\alpha$ que correspondería al valor de $\mathrm{d} \omega / \mathrm{dp}_{\exp }$ obtenido en el ensayo de presión hidrostática, se ajustó el campo de deformaciones obtenido en el modelo numérico para distintos valores de $\alpha$ ( $\beta$ fijo e igual a 0 ) y para a=3 $\mu \mathrm{m}$ y $b=7.5 \mu \mathrm{m}$ con el corrimiento Raman experimental del cuadrante $\mathrm{C}_{2}$. De esta forma, se obtuvieron distintos valores de $e_{4}, f_{4}$ y por consiguiente de $d \omega / d p_{\text {num }}$ para los distintos parámetros de expansión $\alpha$ evaluados. Esto, se presenta en el gráfico de la Figura 62. En la misma, se observa lo siguiente: teniendo en cuenta la expansión que, según hemos estudiado, se aproxima a los modos guiados experimentales: $\alpha=0.01, \beta=0$, corresponde 
un $d \omega / d p \approx 4 \mathrm{~cm}^{-1} / \mathrm{GPa}$ (Marca roja en Figura 62). Este valor es varias veces mayor al valor de $d \omega / d p_{\mathrm{exp}} \approx 1 \mathrm{~cm}^{-1} / \mathrm{GPa}$ medido experimentalmente. Por el contrario, si fijamos dicho valor de $d \omega / \mathrm{dp}_{\mathrm{exp}}$, se obtiene $\alpha=0.04$ (Marca celeste en la Figura 62). En este caso, si aplicamos esta expansión en el modelo numérico, se obtiene una distribución de intensidades para los modos guiados muy distinta a la distribución de intensidades medida experimentalmente (se puede ver la Figura 58 para corroborar esto).

Por otro lado, transcurriendo el año 2013, G. Pezzotti reportó valores de e y $f$ para el fonón $A_{1}(T O)_{4}$ y para otros fonones de especies $E(T O)$ y $A_{2}(T O)$ del niobato de litio puro. Los valores correspondientes al fonón $A_{1}(T O)_{4}$ fueron obtenidos por un método denominado "Crack tip method" que involucra una indentación instrumentada y su modelado utilizando el método de elementos finitos, aplicando una metodología similar a la utilizada en el presente trabajo [PE13]. Reemplazando los valores de $e_{4} \mathrm{y}_{4}$ obtenidos por G. Pezzotti, y las constantes elásticas del niobato de litio, en las Ecuaciones 33, 34 y 36 , se obtiene $d \omega / d p_{G P} \approx 3 \mathrm{~cm}^{-1} / \mathrm{GPa}$. Este valor se encuentra cercano al de $d \omega / d p$ obtenido en el presente estudio para $\alpha=0.01$, que de ahora en adelante se denominará $d \omega / d p_{M G}$. Por lo tanto, a pesar de que el valor de $d \omega / d p_{\exp }$ sirvió en el análisis unidimensional, en una primera aproximación, a este nivel, se consideró que el resultado del experimento hidrostático posee diferencias con el estado de presión no-hidrostático estudiado en las guías, tanto para el análisis actual como en el trabajo mencionado [PE13]. No se conoce con certeza la razón de esta disidencia. Se estima que la misma se debe a que el rango de presiones presente en la guía de onda es menor al utilizado en el ensayo bajo presión hidrostática. Donde, para presiones menores a $0.5 \mathrm{Gpa}$, las incertezas experimentales resultan insuficientes para definir una pendiente $\mathrm{d} \omega / \mathrm{dp}$ debido a la gran dispersión de los puntos, como se mencionó al final de la Sección 5.1. Los corrimientos Raman en este rango de presión hidrostática se estudiarán en el futuro, con quien realizó la medida de espectroscopía $\mu$-Raman bajo presión hidrostática presentada en la Sección 5.1, el Dr. A. Goñi. En tal sentido, se evaluarán distintas variantes de la técnica experimental utilizada que permitan obtener coeficientes de presión ( $\mathrm{d} \omega / \mathrm{dp}_{\exp }$ ) para presiones menores a $0.5 \mathrm{Gpa}$.

Teniendo en cuenta lo mencionado en el párrafo anterior, se consideró apropiado definir los valores de e y $f$ obtenidos de la expansión $\alpha=0.01$ y $\beta=0\left(\mathrm{~d} \omega / \mathrm{dp}_{\mathrm{MG}} \approx 4 \mathrm{~cm}^{-1} / \mathrm{GPa}\right)$ en este estudio bidimensional. 


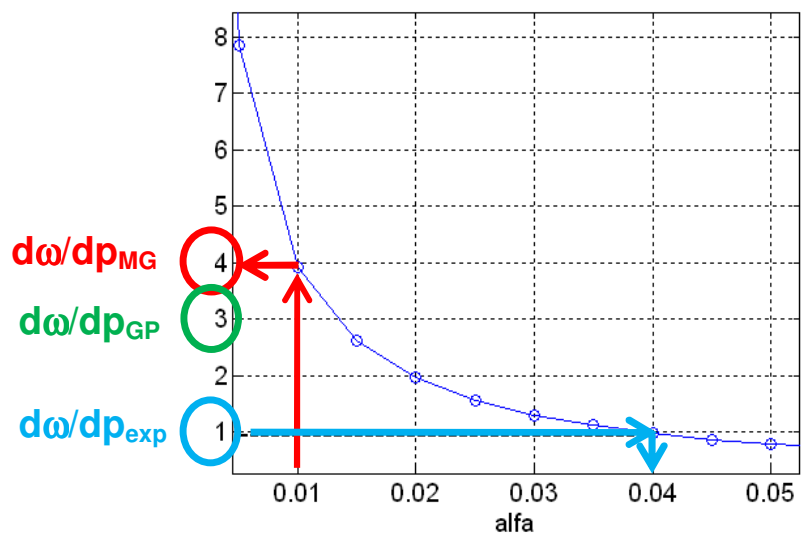

Figura 62. $d \omega / d p$ vs. $\alpha(\beta=0)$

\section{Obtención de Constantes de Deformación Potencial (e y f)}

Utilizando los parámetros del modelo numérico obtenidos como óptimos al ajustar el mapa de corrimiento espectral del fonón $\mathrm{A}_{1}(\mathrm{TO})_{4}$ en el cuadrante $\mathrm{C}_{2}: \mathrm{a}=3, \mathrm{~b}=7.5, \alpha=0.01$ $\beta=0$ y los corrimientos espectrales medidos en el cuadrante $C_{2}$ de los fonones $A_{1}(T O)_{1}$ y $A_{1}(T O)_{2}$, se obtuvieron los valores de e y $f$ mostrados en la Tabla ix. En la misma, también se presentan los valores de $\mathrm{e}_{4} \mathrm{y}_{4}{ }^{8}$ obtenidos por $\mathrm{G}$. Pezzotti y colaboradores [PE13] para su comparación.

\begin{tabular}{|c|c|c|c|c|c|c|}
\hline Fonón & Estudio & $\boldsymbol{e}$ & $\boldsymbol{f}$ & $\boldsymbol{f} / \mathbf{e}$ & $\mathbf{d \omega} / \mathbf{d p _ { \text { num } }}$ & $\mathbf{d \omega / d p _ { \text { exp } }}$ \\
\hline $\mathrm{A}_{1}(\mathrm{TO})_{1}$ & 2D & $-270 \pm 70$ & $-13 \pm 5$ & 0.05 & 1.7 & 0.7 \\
\hline $\mathrm{A}_{1}(\mathrm{TO})_{2}$ & 2D & $-230 \pm 60$ & $-215 \pm 50$ & 0.9 & 2 & 5.5 \\
\hline $\mathrm{A}_{1}(\mathrm{TO})_{4}$ & 2D & $-550 \pm 130$ & $-160 \pm 40$ & 0.3 & 4 & 1 \\
\cline { 2 - 7 } & G.Pezzotti[PE13] & $-450 \pm 93$ & $-195 \pm 52$ & 0.4 & 3 & \\
\hline
\end{tabular}

Tabla ix. Valores de $\boldsymbol{e}, \boldsymbol{f}, \boldsymbol{f} / \mathbf{e}$ y $\boldsymbol{d} \boldsymbol{\omega} / \boldsymbol{d} \boldsymbol{p}$ obtenidas en el estudio bidimensional.

En la Figura 63 se presentan los ajustes numéricos y medidas experimentales de los corrimientos de los fonones $A_{1}(T O)_{1}$ y $A_{1}(T O)_{2}$ para el cuadrante $C_{2}$. Aunque las medidas experimentales tienen una baja relación señal-ruido, se puede observar que concuerdan con el resultado numérico.

${ }^{8}$ Cuando se haga referencia a los valores de e y $f$ de un determinado fonón $\mathrm{A}_{1}(\mathrm{TO}) \mathrm{i}$, se utilizará el subíndice "i": $e_{i}$ y $f_{i}$. 


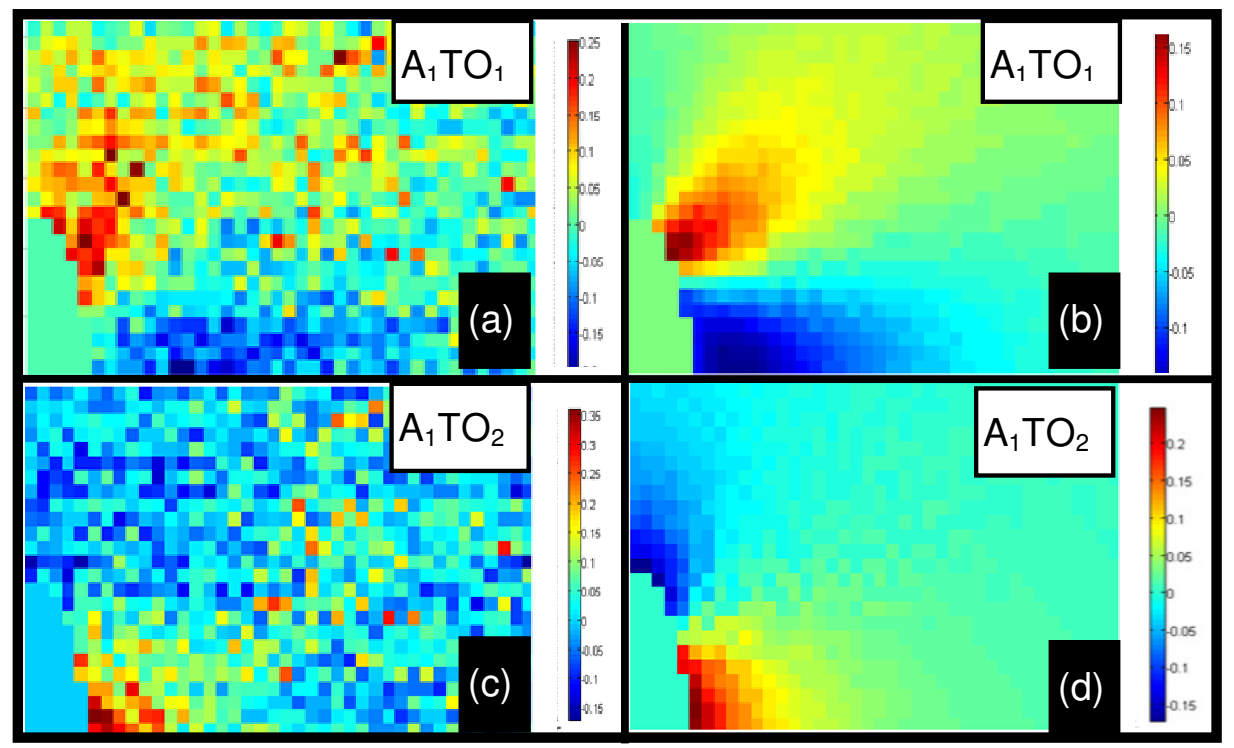

Figura 63. (a) Mapa $\mu$-Raman experimental en cuadrante $C_{2}$ de fonón $A_{1}(T O)_{1}$, (b) Ajuste al corrimiento $\mu$-Raman de fonón $A_{1}(T O)_{1}$, (c) mapa $\mu$-Raman experimental en cuadrante $\mathrm{C}_{2}$ de fonón $\mathrm{A}_{1}(\mathrm{TO})_{2}$, (d) Ajuste al corrimiento $\mu$-Raman de fonón $\mathrm{A}_{1}(\mathrm{TO})_{2}$

Para el fonón $\mathrm{A}_{1} \mathrm{TO}_{4}$, se puede observar en la Tabla ix lo siguiente:

- Los valores de $e_{4}$ y $f_{4}$ son negativos en el presente estudio y en el reportado por Pezzotti.

- En ambos estudios $\left|e_{4}\right|>\left|f_{4}\right|$.

- Los valores de $d \omega / d p_{\text {num }}$ son tres y cuatro veces mayores al $d \omega / d p_{\exp }$ medido en el ensayo bajo presión hidrostática, para el estudio de Pezzotti y el actual, respectivamente.

Esta última discrepancia, incita a profundizar, en trabajos futuros, sobre el método experimental empleado para obtener el coeficiente de presión hidrostático ( $\left.d \omega / \mathrm{dp}_{\mathrm{exp}}\right)$.

En cuanto a los valores de $d \omega / d p_{\text {num }}$ obtenidos para los fonones $A_{1}(T O)_{1}$ y $A_{1}(T O)_{2}$, se puede observar que el primero duplica su correspondiente valor experimental de $\mathrm{d} \omega / \mathrm{dp}_{\mathrm{exp}}$, mientras que el segundo es igual a la mitad de su correspondiente valor de $\mathrm{d} \omega / \mathrm{dp} \mathrm{p}_{\exp }$.

En las siguientes secciones, se utilizarán los valores de e y $f$ obtenidos del ajuste bidimensional y presentados en la Tabla ix para estudiar el comportamiento del campo de deformaciones, a partir de los mapas de corrimiento en número de onda de los fonones $\mathrm{A}_{1}(\mathrm{TO})_{1}, \mathrm{~A}_{2}(\mathrm{TO})_{2}$ y $\mathrm{A}_{1}(\mathrm{TO})_{4}$. 


\subsection{Cálculo directo de deformaciones, índice de refracción e intensidad guiada utilizando $\mu$-Raman}

La aplicación del análisis bidimensional anterior para la caracterización de guías de onda, requiere numerosos pasos de cálculo para obtener los parámetros del modelo que permitan representar de forma más adecuada la expansión originada en la interacción. Este procedimiento es requerido aún conociendo previamente los valores de e y $f$ del material. A su vez, dicho análisis tiene robustez limitada, ya que si la guía de onda no es originada por una expansión similar a la propuesta en el modelo numérico, como ocurre en la guía a la derecha de la modificación $B$, resulta de mayor dificultad su aplicación. Por tal motivo, asumiendo conocidos los valores de e y $f$ del cristal para los fonones $\mathrm{A}_{1}(\mathrm{TO})_{1}$, $A_{1}(T O)_{2}$ y $A_{1}(T O)_{4}$, se propone un método directo para obtener el campo de deformaciones y su campo de variación de índice refracción asociado. Este método directo, se basa en la utilización de los mapas de corrimiento Raman en la zona de guiado de luz y las Constantes de Deformación Potencial ( $e$ y $f$ ), previamente determinadas, de cualquier par de fonones $A_{1}(T O)$.

En un primer paso, para calcular en forma directa los campos de deformaciones $\varepsilon_{\mathrm{xc}}$ y $\varepsilon_{\mathbf{z c}}$ en la zona que se realizó el mapa de corrimiento Raman, se utilizaron las ecuaciones que brinda la Teoría de Deformación Potencial Lineal para cada fonón $A_{1}(T O)$. Ellas son las siguientes (se asume deformaciones planas, $\varepsilon_{\mathrm{yc}}=0$ ):

$$
\begin{aligned}
& e_{1} \varepsilon_{x c}(z, x)+f_{1} \varepsilon_{z c}(z, x)=\Delta \omega_{1}(z, x) \\
& e_{2} \varepsilon_{x c}(z, x)+f_{2} \varepsilon_{z c}(z, x)=\Delta \omega_{2}(z, x) \\
& e_{4} \varepsilon_{x c}(z, x)+f_{4} \varepsilon_{z c}(z, x)=\Delta \omega_{4}(z, x)
\end{aligned}
$$

La Ecuación 74 corresponde al fonón $A_{1}(\mathrm{TO})_{1}$, la Ecuación 75 al fonón $A_{1}(\mathrm{TO})_{2}$ y la Ecuación 76 al fonón $A_{1}(T O)_{4}: \Delta \omega_{i}\left(Z_{c}, x_{c}\right)$ es el mapa de corrimiento espectral de cada fonón respecto de su valor nominal, $e_{i}$ y $f_{i}$, las Constantes de Deformación Potencial correspondientes a cada fonón $A_{1}(T O)$.

El método directo de cálculo de deformaciones consiste en resolver, en cada coordenada espacial del mapa $\left(z_{c}, x_{c}\right)$, un sistema de ecuaciones formado por dos de las ecuaciones precedentes. De esta forma, se obtienen las deformaciones $\varepsilon_{\mathrm{xc}}$ y $\varepsilon_{z \mathrm{c}}$ en cada punto $\left(z_{\mathrm{ci}}, \mathrm{x}_{\mathrm{ci}}\right)$ del mapa. Luego se calcula, aplicando la relación piezo-óptica mediante el código campoind2.m (Anexo B), el campo de índice de refracción generado por estas deformaciones. A partir de estos campos de índice de refracción obtenidos, se calcularon las distribuciones de intensidad guiada correspondientes a la variación de la componente vertical y horizontal del índice de refracción $\left(\Delta n_{x c}\right.$ y $\Delta n_{z c}$ ), utilizando el código codigo3.m (Anexo B). Las mismas se compararon con las medidas experimentales. 


\subsubsection{Modificación B}

Para aplicar el método directo mencionado, a los mapas de corrimientos Raman obtenidos en la modificación $B$, se eligió arbitrariamente el par de fonones $A_{1}(T O)_{2^{-}}$ $\mathrm{A} 1(\mathrm{TO})_{4}$ para realizar un primer cálculo del campo de deformaciones. En la siguiente Sección, se estudiará la aplicación de este método para las diferentes combinaciones posibles de fonones. En la Figura 64, se presentan los campos de deformaciones y de índice de refracción obtenidos aplicando el método directo propuesto. Se utilizaron los valores de $e$ y $f$ presentados en la Tabla ix

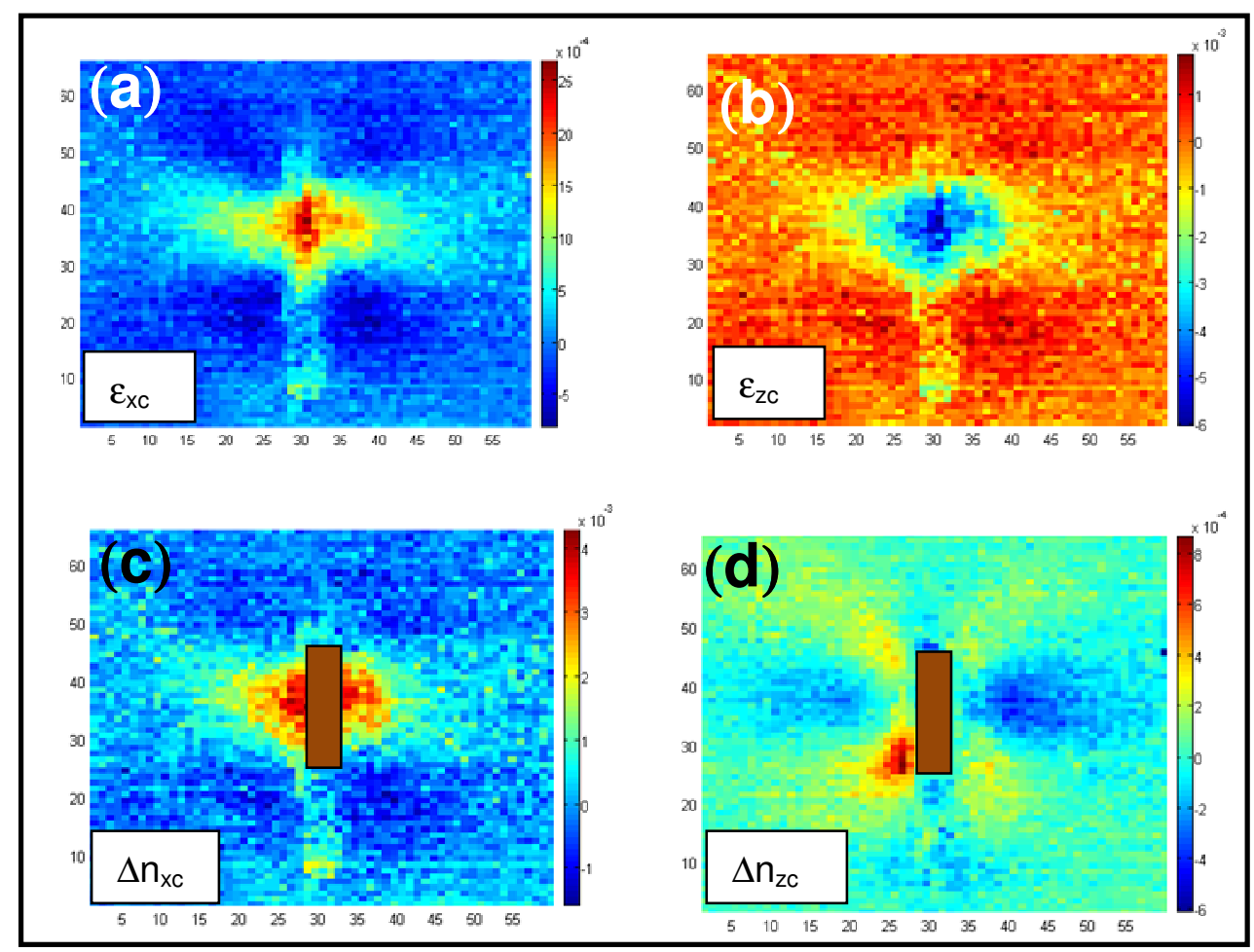

Figura 64. Campos de deformaciones (a) $\varepsilon_{x c} \mathbf{y}$ (b) $\varepsilon_{z c}$ y campo de variación de índice de refracción (c) $\Delta n_{x} y$ (d) $\Delta n_{z}$ calculados de los mapas Raman por el método directo

Una vez calculado el campo de índice de refracción (Figura 64(c) y (d)), se observó que en la zona de modificación estructural ó zona central, el índice de refracción aumenta considerablemente utilizando el par de fonones elegido. Esto no es físicamente posible, ya que no es posible guiar luz en esta región y experimentalmente se observa una disminución abrupta de índice de refracción en la misma. Por tal motivo, para calcular los modos guiados en las adyacencias de la zona central se colocó arbitrariamente un escalón de índice (por simplicidad rectangular, este se observa en la Figura 64(c)) de $\Delta \mathrm{n}=-1 \times 10^{-2}$. Estos modos guiados, se calcularon para ambas polarizaciones guiadas $\left(\mathrm{x}_{\mathrm{c}}\right.$ y $z_{c}$ ) cuya suma de intensidades se presenta en la Figura 65. Cabe mencionar que la principal componente de los modos es en la dirección vertical $\left(x_{c}\right)$. Esto, se debe a que el 
incremento de índice de refracción obtenido para esta componente es aproximadamente cinco veces mayor que aquel obtenido para la polarización horizontal.

En la Figura 65, se pueden comparar los modos calculados a partir de los mapas de corrimiento Raman con los medidos experimentalmente para una longitud de onda propagada de $650 \mathrm{~nm}$. Se observa que la zona de guiado es aproximadamente coincidente, aunque los modos calculados no se aproximan a los modos experimentales como lo hacen aquellos obtenidos con el modelo elástico de deformaciones, en la sección anterior. Se puede observar que los modos calculados tiene una zona cercana a la modificación donde la intensidad se eleva bruscamente. Esto se debe a que el material se encuentre modificado estructuralmente o demasiado deformado en esta región. Por lo tanto, la Teoría de Deformación Potencial Lineal, para el par de fonones utilizados, no es válida en esta región. La aplicación de este método utilizando los otros pares de fonones se estudia en la próxima sección.

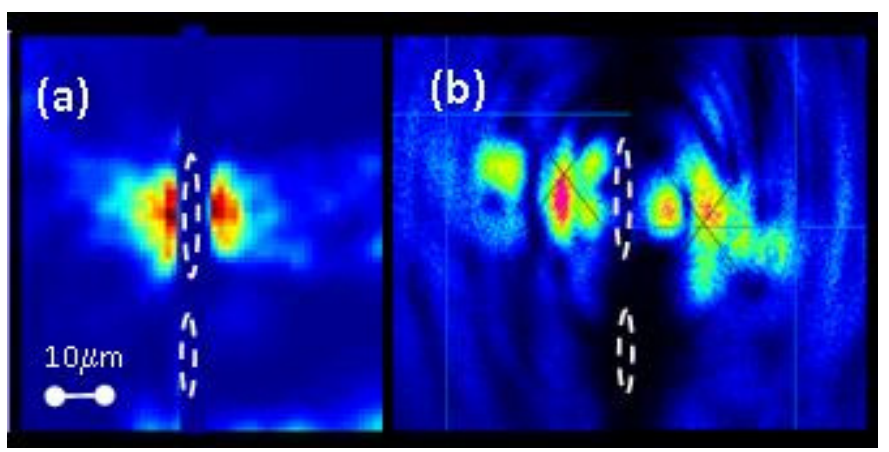

Figura 65. Modos de propagación para $\lambda=650 \mathrm{~nm}$ : (a) calculados a partir de los mapas Raman utilizando el método directo (b) Medidos experimentalmente sin polarizadores. La escala de la intensidad es arbitraria en ambas figuras y la escala espacial es la misma en ambas

\subsubsection{Cálculo directo de modos en guías de onda de doble modificación}

Se calcularon los modos de propagación de forma directa para las guías lindantes a las modificaciones C, mostradas nuevamente en la Figura 66(a). Para realizar esto, se siguieron los pasos indicados en el apartado anterior. Las estructuras C, están conformadas por dos modificaciones paralelas separadas por $20 \mu \mathrm{m}$.

En la Figura 66(b) y (c) se presentan los modos guiados medidos experimentalmente en las adyacencias de las modificaciones para $\lambda=650 \mathrm{~nm}$ y $\lambda=1550 \mathrm{~nm}$, respectivamente. Se pueden observar tres guías ó zonas de guiado principales: a la izquierda, en la zona central y a la derecha. Las distribuciones de intensidad no son completamente simétricas en las diferentes regiones (guías). Las imágenes presentadas en las Figura 66(b) y (c) se generaron superponiendo las distribuciones de intensidad medidas en cada guía individualmente. En la Figura 66(b), la distribución de intensidad guiada a la derecha de 
las modificaciones estructurales se ve modificada por imperfecciones del material, que generan cierta dispersión en la intensidad. Esta zona se encierra con la curva verde de a trazos A.

En las adyacencias de las modificaciones menores (o filamentaciones) también se han detectado zonas de guiado aunque de menor intensidad, lo que se correlaciona con un menor incremento de índice de refracción.

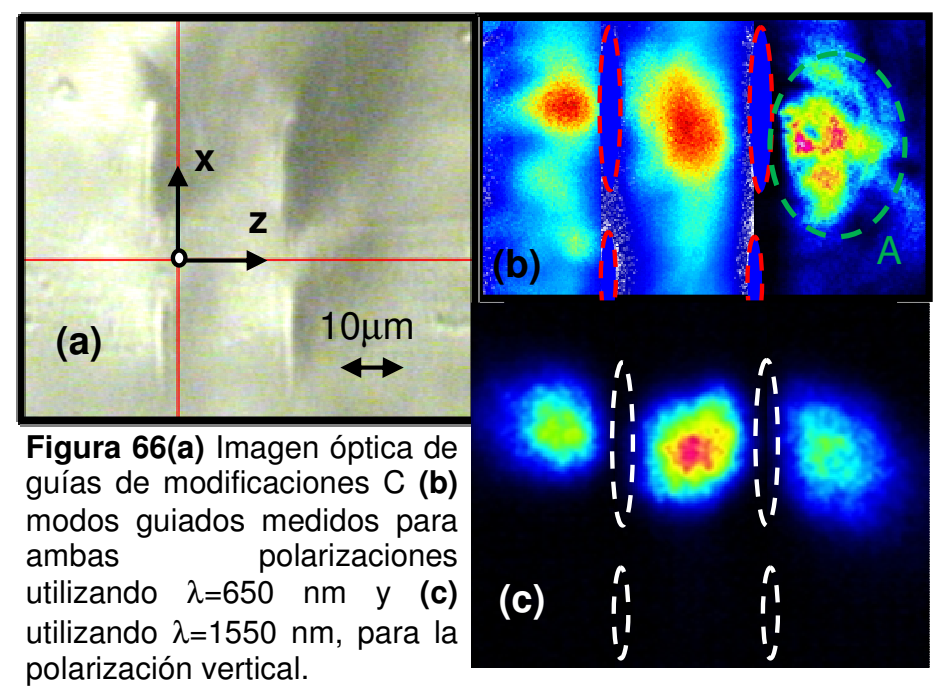

Luego, se realizó un mapa $\mu$-Raman en estas guías. Los corrimientos de los fonones $A_{1}(T O)_{1}, A_{1}(T O)_{2}$ y $A_{1}(T O)_{4}$ se presentan en la Figura 67. En la Figura 67(d) se presenta la variación de intensidad del fonón $A_{1}(T O)_{4}$. Para el fonón $A_{1}(T O)_{3}$ no se detectó señal. En estos mapas, se puede observar un comportamiento análogo al medido en la guía simple (modificación B) para las zonas cercanas a cada modificación estructural, aunque con mejor relación señal-ruido. En particular, el mapa correspondiente al fonón $A_{1}(T O)_{2}$ tiene un comportamiento más homogéneo que su homólogo para la guía de simple modificación. La zona oscura en el mapa de variación de intensidad del fonón $A_{1}(T O)_{4}$, mostrado en la Figura 67(d), se utilizó para definir el tamaño de la zona rectangular central con disminución del índice de refracción. A esta zona de bajo índice de refracción se le asignó un valor constante de $\Delta n=-1 \times 10^{-2}$. Este valor es medido comúnmente para este tipo de guías [BIA13] y por otro lado no tiene implicancia significativa en la forma de los modos guiados ya que la zona de modificación estructural es adyacente a la guía. 


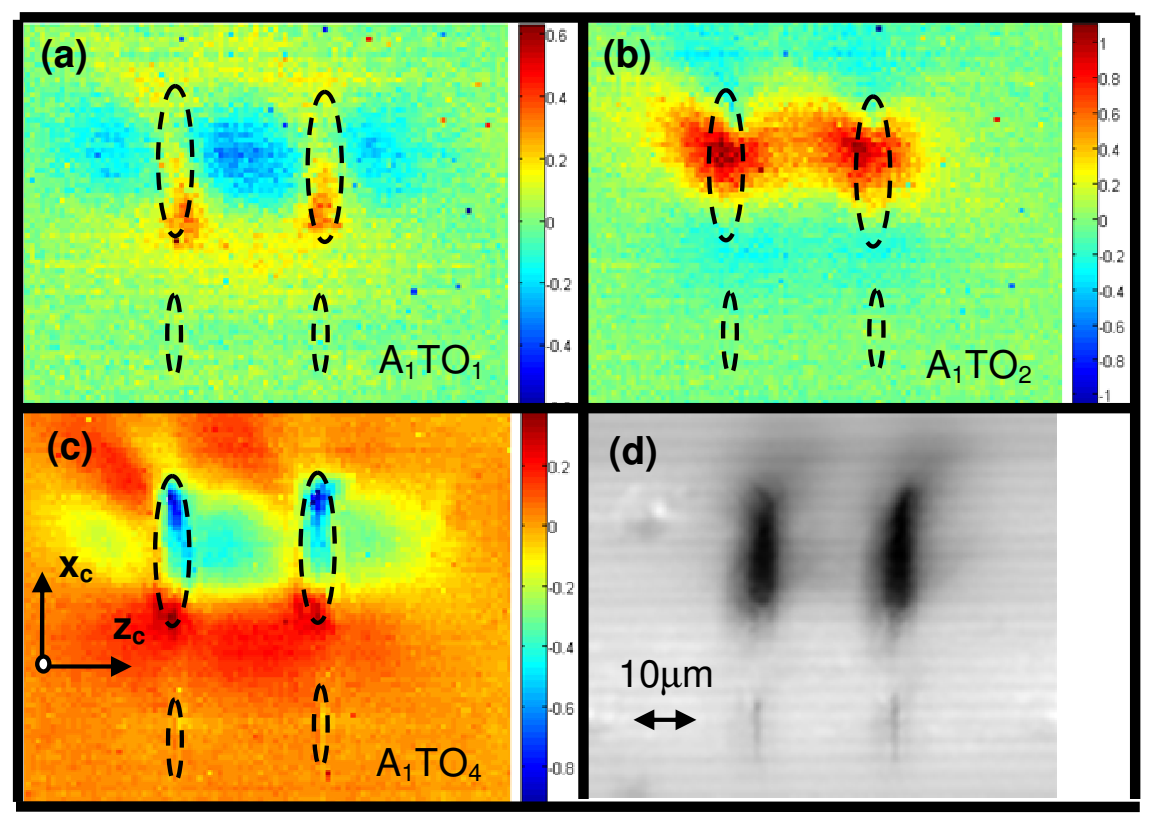

Figura 67. Corrimientos espectrales Raman: (a) fonón $A_{1}(T O)_{1}$, (b) fonón $A_{1}(T O)_{2}$, (c) fonón $A_{1}(T O)_{4}$, (d) variación de intensidad del fonón $A_{1}(T O)_{4}$

Aplicando la metodología de cálculo directo descrita al comienzo de la Sección 5.3, se calcularon los campos de deformaciones y los campos de variación de índice de refracción para cada combinación posible de fonones y asumiendo los valores de e y $f$ presentados en la Tabla ix. En la Figura 68(a), (b) y (c) se presentan los campos de índice de refracción $\Delta \mathrm{n}_{\mathrm{xc}}$ obtenidos. Por fuera de la zona central de modificación directa, los tres pares de fonones permiten obtener un aumento y una disminución de índice de refracción en las mismas zonas. Dentro de la zona central de modificación directa, para los pares $A_{1}(T O)_{1}-A_{1}(T O)_{2}$ y $A_{1}(T O)_{2}-A_{1}(T O)_{4}$ se puede ver que ambos campos de índice de refracción obtenidos observan un incremento, en lugar de una disminución. Lo que no es físicamente posible. Por lo tanto, estos fonones no son apropiados en esta zona para calcular la variación de índice de refracción en esta zona. Entonces, para el cálculo de los modos guiados en las zonas adyacentes a la modificación estructural, las zonas de disminución de índice de refracción se asumieron rectangulares y de una magnitud igual a $-1 \times 10^{-2}$ para los pares $A_{1}(T O)_{1}-A_{1}(T O)_{2}$ y $A_{1}(T O)_{2}-A_{1}(T O)_{4}$. Por el contrario, para el par $A_{1}(T O)_{4}-A_{1}(T O)_{1}$ no fue necesario forzar esa disminución de índice de refracción. El campo de variación de índice de refracción (componente $n_{x}$ ) obtenido a partir de este par de fonones $\left(\mathrm{A}_{1}(\mathrm{TO})_{1}-\mathrm{A}_{1}(\mathrm{TO})_{4}\right)$, sigue el comportamiento esperado para la zona de modificación estructural: decrece hasta $\Delta \mathrm{n}=-1 \times 10^{-2}$ (Figura 68(c)). Esto indica qué, en la zona de interacción directa (estructuralmente modificada) esta combinación de fonones es la única que permite estimar el cambio de índice. Además, se puede ver que a pesar de ser el campo de índice de refracción de menor relación señal-ruido, es el que mejor ajusta a la intensidad medida. Principalmente, porque los otros campos de índice predicen modos guiados con su valor máximo demasiado cerca a la modificación para ambas longitudes de onda (Figura 68( $\left.\left(e_{1}\right),\left(e_{2}\right),\left(f_{1}\right),\left(f_{2}\right)\right)$. En cambio, el campo de índice 
de refracción obtenido por el par de fonones $A_{1}(T O)_{1}-A_{1}(T O)_{4}$ predice los máximos de la intensidad guiada más alejados de la modificación (Figura $68\left(\mathrm{f}_{1}\right)$ y $\left(\mathrm{f}_{2}\right)$ ), que es lo observado en los modos experimentales. Para ver esto, se puede comparar, por ejemplo, la región encerrada por la curva B en la Figura $68\left(\mathrm{f}_{2}\right)$ y aquella encerrada por la curva B' en la Figura $68(\mathrm{~g})$. A su vez, el campo de $\Delta \mathrm{n}_{\mathrm{xc}}$ obtenido utilizando este par de fonones $\left(A_{1}(T O)_{1}-A_{1}(T O)_{4}\right)$, es el único que predice la dispersión de la intensidad guiada a la derecha de las modificaciones, de característica similar a la medida experimentalmente para $\lambda=650 \mathrm{~nm}$. Esta característica se observa comparando la región encerrada por la curva A en la Figura $68\left(f_{1}\right)$ y aquella encerrada por la curva A' en la Figura 68(h).

Los modos guiados se calcularon para la variación $\Delta \mathrm{n}_{\mathrm{xc}}$ porque, como ya se mencionó, esta es la componente principal que permite el guiado de luz en este tipo de guías. Las longitudes de onda implementadas en el cálculo numérico y en las medidas experimentales son de 650 y $1550 \mathrm{~nm}$. En general, la distribución de intensidad calculada para $\lambda=650 \mathrm{~nm}$ tienen mayor magnitud que aquella correspondiente a $\lambda=1550$ $\mathrm{nm}$ (por ejemplo: 0.25 vs 0 . 04). Esto, también es observable en las medidas experimentales. Para esta última longitud de onda, se necesita mayor amplificación en la colección de la imagen y/ó mayor intensidad del láser acoplado para obtener una señal aceptable de la distribución de intensidad guiada.

Las regiones de incremento de índice de refracción obtenidas con este método directo para los distintos pares de fonones, coinciden cualitativamente, con las medidas experimentales. A su vez, las características de las distribuciones de intensidad numéricas obtenidas utilizando el par de fonones $A_{1}(T O)_{1}-A_{1}(T O)_{4}$ es la que tiene mayor correlación con las distribuciones de intensidad medidas experimentalmente. Cabe resaltar que este método permite, de una manera simple y de un solo paso numérico, obtener el campo de variación de índice de refracción de guías originadas por deformaciones. 

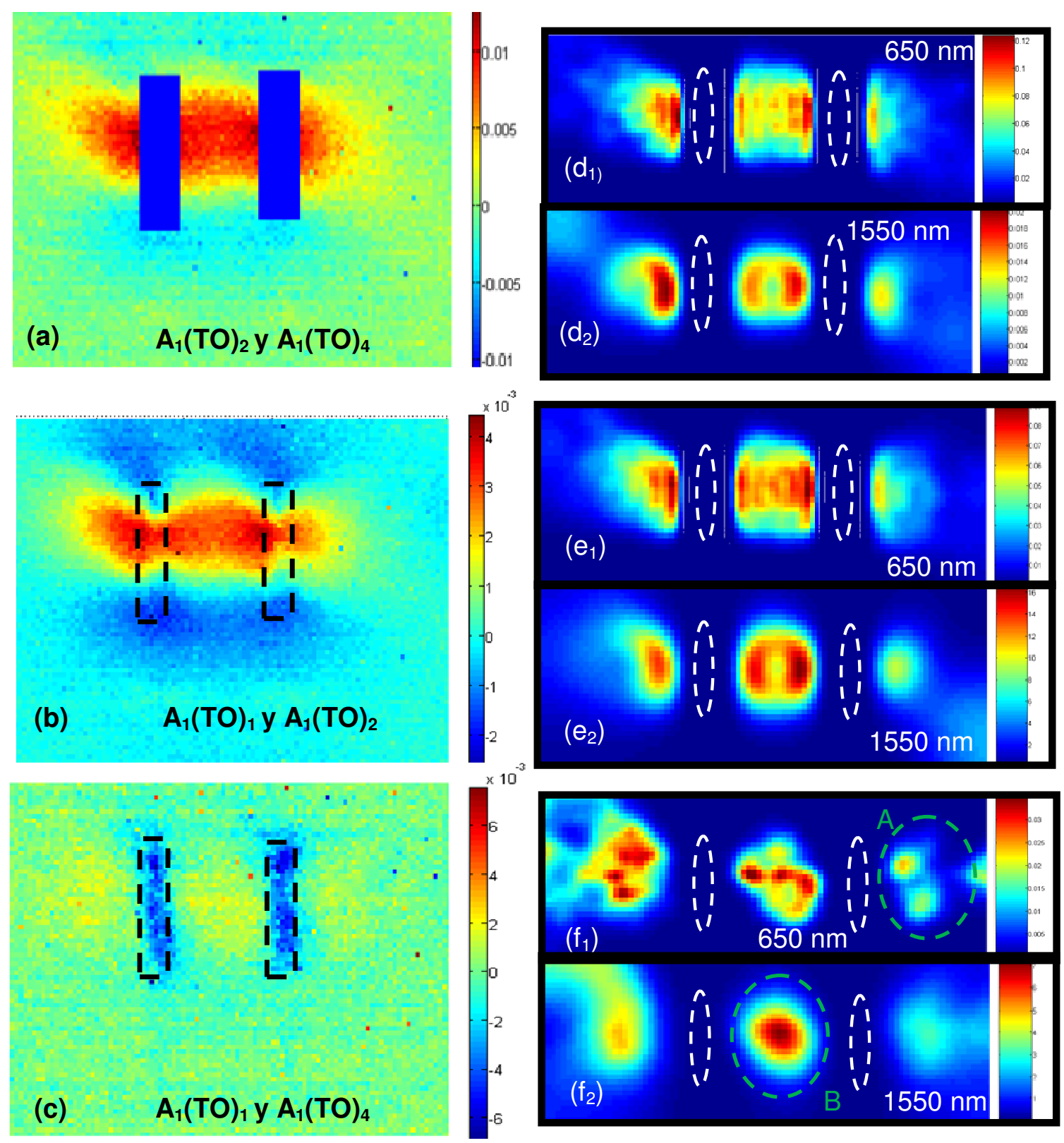

\section{Modos guiados experimentales}
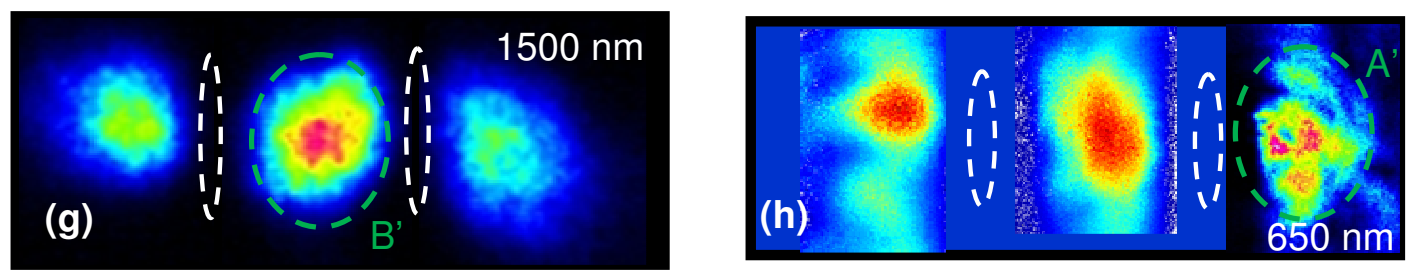

Figura 68. Campo de índice de refracción $\left(\Delta \mathrm{n}_{\mathrm{xc}}\right)$ para los tres pares de fonones: (a), (b) y (c). Modos guiados numéricos para los diferentes pares de fonones y para $\lambda=650 \mathrm{~nm}$ y $\lambda=1550 \mathrm{~nm}$ (d1), (d2), (e1), (e2), (f1) y (f2). Modos guiados medidos experimentalmente: (g) $1550 \mathrm{~nm}$ y (h) $650 \mathrm{~nm}$ 


\subsection{Polarización de luz guiada en modificaciones fabricadas con láser de femtosegundos de diferentes energías de pulso}

En cuanto a la polarización de la luz guiada por guías de onda fabricadas con pulsos láser de femtosegundos en niobato de litio, existe cierta ambigüedad en la literatura. En algunos trabajos, se ha reportado que estas estructuras sólo guían la polarización vertical [BUR07] (dirección $x_{c}$ ) mientras que en otros trabajos se reporta el guiado de luz de ambas polarizaciones [RO09, BURHA07]. En esta Sección, se presenta un estudio de la polarización de la intensidad guiada en distintas guías de onda fabricadas con láser de femtosegundos por el autor.

\subsubsection{Comparación cualitativa del modelo numérico con medidas experimentales previas}

Se ha reportado en algunos trabajos [RO09, BURHA07], que las modificaciones estructurales fabricadas desplazando un láser de femtosegundos en dirección $\mathrm{y}_{\mathrm{c}} \mathrm{y}$ enfocando en la dirección $x_{c}$ en un cristal de niobato de litio, también guían luz polarizada en la dirección horizontal (eje $z_{c}$ ). Para analizar este fenómeno, de forma cualitativa, se compararon los campos de variación de índice de refracción resultantes de distintas expansiones numéricas representativas, con medidas experimentales reportadas por J. Burghoff y colaboradores [BURHA07].

Los campos de índice de refracción obtenidos con el modelo numérico $\left(\Delta \mathrm{n}_{\mathrm{xc}}\right.$ y $\left.\Delta \mathrm{n}_{\mathrm{zc}}\right)$ para tres expansiones representativas (utilizando una elipse de dimensiones arbitrarias), se presentan en la Figura 69. En esta, se puede ver que la expansión que genera aumento de índice de refracción para las dos polarizaciones guiadas $\left(x_{c} \quad y z_{c}\right)$ en las adyacencias de la elipse, es la expansión $1(\alpha=0.01, \beta=0.0)$. Esta región se encierra con una curva a trazos en la Figura 69(a). En cambio, las expansiones $2(\alpha=\beta=0.02)$ y $3(\alpha=0, \beta=0.02)$, sólo generan aumento de la componente $x_{c}$ (dirección vertical) del índice de refracción en las adyacencias de la modificación estructural, mientras que el incremento de la componente $z_{c}$ del índice de refracción se produce en la parte superior de la elipse.

Burghoff y colaboradores [BURHA07] reportaron la distribución de intensidad guiada de polarización $x_{c} \quad y \quad z_{c}$. Dicho estudio, se realizó en guías fabricadas con láser de femtosegundos, en un cristal de niobato de litio corte $X$ para tiempos de pulso de hasta 1 ps y para energías de pulso iguales a $0.2 \mu \mathrm{J}$. Las intensidades guiadas reportadas en este trabajo son, en general, simétricas. En la Figura 70, se puede ver la concordancia entre las regiones de aumento de índice de refracción generadas, para ambas polarizaciones, por la expansión 1 y la distribución de intensidades experimental reportada. La luz guiada de polarización horizontal $\left(z_{c}\right)$ experimental, tiene forma de "herradura" y es común para todos los tiempos de pulso (aunque se visualiza con mayor 
claridad para $1 \mathrm{ps})$. A su vez, el aumento de índice de refracción en la componente $\mathrm{z}_{\mathrm{c}}$ obtenido en el modelo numérico para la expansión 1, tiene geometría similar. Al mismo tiempo, la región donde la luz guiada es de polarización vertical $\left(x_{c}\right)$ coincide, aproximadamente, con el aumento de índice de refracción (componente $x_{c}$ ) resultante para la expansión 1 del modelo numérico. Por otro lado, se puede ver en la Figura 70(a) que para pulsos de $300 \mathrm{fs}$, la luz de polarización $x_{c}$ guiada experimental es de mayor intensidad que la de polarización $z_{c}$. Esto también tiene correlato con la expansión "horizontal" (en dirección $z_{c}$ ) en el modelo numérico propuesto en este trabajo, ya que el incremento de la componente $Z_{c}$ del índice de refracción es aproximadamente 4 veces menor que el aumento de índice para la polarización $X_{c}$ en el modelo numérico(Figura 69).

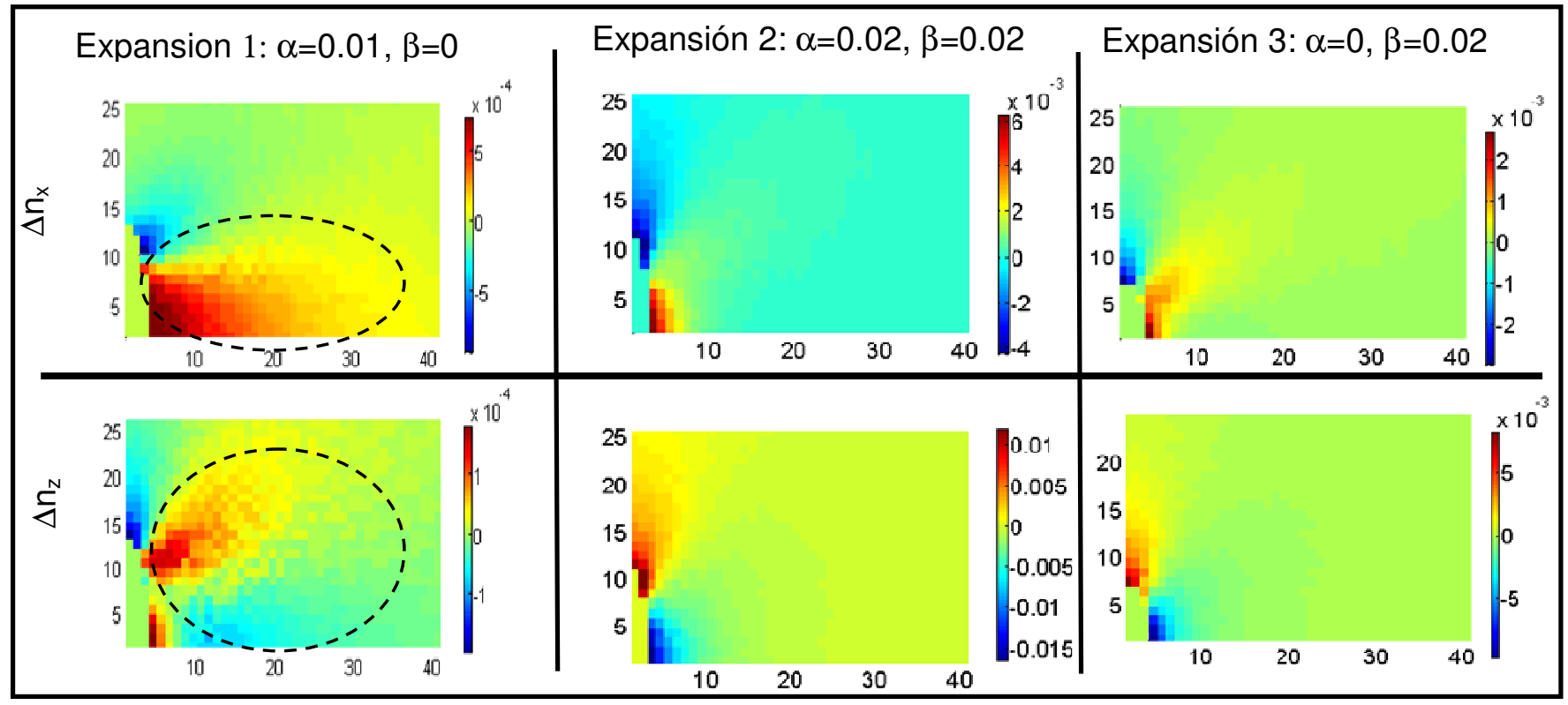

Figura 69. Campo de índice de refracción numérico, componentes $\Delta \mathrm{n}_{\mathrm{xc}}$ y $\Delta \mathrm{n}_{\mathrm{zc}}$ para distintas expansiones representativas

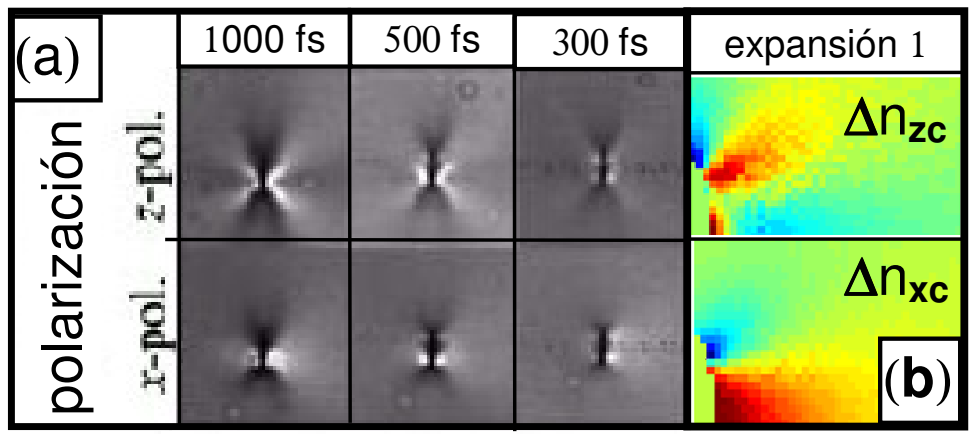

Figura 70. (a) Modos guiados experimentales para diferentes tiempos de pulso en polarización z y polarización x para una energía de pulso de $0.2 \mu \mathrm{J}$. (b) Campo de índice numérico para expansión 1. 
Con respecto a la polarización de la luz guiada en este tipo de guías, la siguiente pregunta se ha presentado en trabajos anteriores [WILL05]: ¿Por qué, siendo que el modelo numérico para la expansión 2 (que es la utilizada en dichos trabajos), predice un aumento de $\Delta \mathrm{n}_{\mathrm{zc}}$ en la región superior a la modificación, en general, no se ha reportado luz guiada en esta zona? La respuesta es que este tipo de expansión no es la que mejor ajusta a la interacción física. Evidentemente, la expansión 1 es la que se corresponde y la misma, no genera aumento de índice de refracción $\Delta \mathrm{n}_{\mathrm{zc}}$ en la zona superior a la elipse, sino que genera una disminución para ambas componentes del índice de refracción $\left(\Delta \mathrm{n}_{\mathrm{xc}}\right.$ y $\Delta \mathrm{n}_{\mathrm{zc}}$ ) en dicha región.

Los párrafos precedentes, sumados a los resultados obtenidos en los análisis unidimensional y bidimensional previos permiten la siguiente generalización: en el tipo de guías estudiadas, para la orientación del cristal de niobato de litio y la dirección incidencia de láser de femto-segundos utilizada, la expansión originada es principalmente horizontal ó coincidente con el eje $z_{c}$. Es decir que la variante introducida durante este trabajo en el modelo numérico; que implica que $\alpha$ y $\beta$ pueden tomar valores sin restricciones preestablecidas; permite explicar un aumento de índice de refracción en la dirección horizontal $\left(\Delta n_{z}\right)$ en zonas adyacentes a la modificación estructural y una disminución del mismo en la zona superior.

Por otro lado, el modelo numérico predice que esta variación es de menor magnitud que la variación del índice de refracción vertical $\left(\Delta n_{\mathrm{xc}}\right)$, lo que generaría modos más extendidos, debido al menor confinamiento. Para aportar más información sobre esta cuestión, se realizó un estudio de la propagación para ambas polarizaciones en estructuras fabricadas con láser de femtosegundos variando los valores de energía de pulso del láser. A continuación, se reporta la fabricación de modificaciones estructurales rectas con pulsos láser de femtosegundos de distintos valores de energía y un análisis de la luz guiada para diferentes polarizaciones en las zonas adyacentes a las modificaciones. Asimismo, se comparan los resultados experimentales con distribuciones de intensidad guiada obtenidas en el modelo numérico.

\subsubsection{Procedimientos experimentales y resultados}

Se fabricaron guías de onda rectas en niobato de litio puro corte $X$ con distintas energías de pulso. Luego se midieron las intensidades guiadas, para las distintas polarizaciones, a ambos lados de cada modificación estructural generada. Para la fabricación de estas guías de onda, se utilizó el sistema de generación de pulsos láser de femtosegundos y mecanizado disponible en el ClOp, descrito en el Capítulo 3. En la Tabla x se presentan los valores de energía utilizados. Estas estructuras fueron fabricadas por el autor de este trabajo de Tesis.

Todas las estructuras se fabricaron empleando pulsos de $150 \mathrm{fs}$, un objetivo de 20x, a una velocidad de traslación de $25 \mu \mathrm{m} / \mathrm{s}$ en dirección " $\mathrm{y+}$ " del cristal y a una profundidad 
nominal de $250 \mu \mathrm{m}$ (en dirección $\mathrm{x}_{\mathrm{c}}$ ). La polarización del láser de femtosegundos utilizada es paralela al eje $z_{c}$ (normal a la dirección de escritura). Las estructuras están separadas $200 \mu \mathrm{m}$ en la dirección $\mathrm{z}_{\mathrm{c}}$.

\begin{tabular}{|c|c|}
\hline $\begin{array}{c}\text { Modifica } \\
\text { ción }\end{array}$ & $\begin{array}{c}\text { Energía de } \\
\text { pulso }\left(\mathrm{e}_{\mathrm{p}}\right)\end{array}$ \\
\hline 1 & $1.2 \mu \mathrm{J}$ \\
\hline 2 & $1.0 \mu \mathrm{J}$ \\
\hline 3 & $0.8 \mu \mathrm{J}$ \\
\hline 4 & $0.6 \mu \mathrm{J}$ \\
\hline 5 & $0.4 \mu \mathrm{J}$ \\
\hline
\end{tabular}

Tabla x. Energía de pulso (e) utilizada para las estructuras generadas

Una vez fabricadas las estructuras, se pulieron los cantos del cristal normales al eje de propagación como se indicó en la Sección 3.

En la Figura 71 se muestran las modificaciones estructurales fabricadas. Se puede observar que la longitud de las mismas se incrementa al incrementar la energía del láser. Para energías menores a $0.4 \mu \mathrm{J}$ no se observó modificación. Además, para las energías mayores (modificaciones 1, 2 y 3), se observa el fenómeno denominado "filamentación" (Capítulo 2), debido al cual la zona modificada se divide en dos regiones.

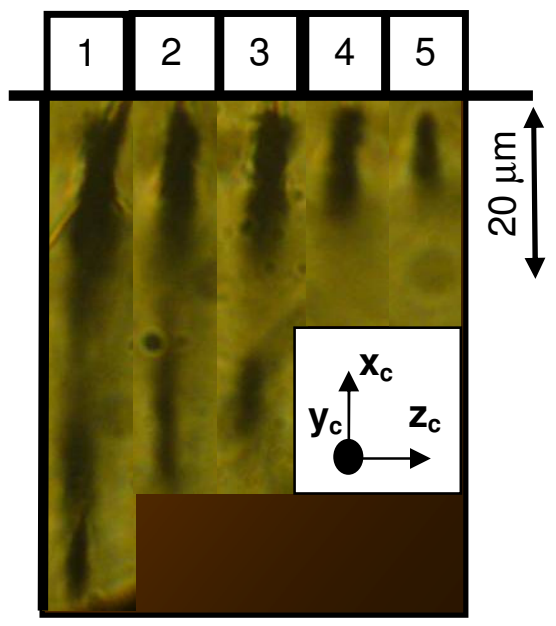

Figura 71.. Imagen óptica microscópica en transmisión de las estructuras fabricadas con el láser de femtosegundos (canto del cristal). El láser incide desde la parte superior. 
Para acoplar las guías de onda y visualizar la intensidad guiada, se utilizaron los dispositivos descritos en el Capítulo 3. La disposición de los mismos se detalla en la Figura 72. El láser, linealmente polarizado, se dispone de forma tal que su polarización esté a aproximadamente $45^{\circ}$ del eje cristalino $z_{c}$. Por lo tanto, al girar el polarizador $A$ se selecciona la componente de polarización requerida (horizontal, eje $\mathrm{z}_{\mathrm{c}}$ o vertical, eje $\mathrm{x}_{\mathrm{c}}$ ). Luego, el polarizador B, ubicado a la salida de la guía, se coloca en la misma dirección que el polarizador A. Esto se hizo para colectar sólo la polarización guiada en la dirección estudiada. Asimismo, no se observó intensidad guiada cuando se colocaron los polarizadores cruzados entre sí 90º, para ambas polarizaciones de entrada. Este experimento fue realizado en el ClOp por el autor de este trabajo.

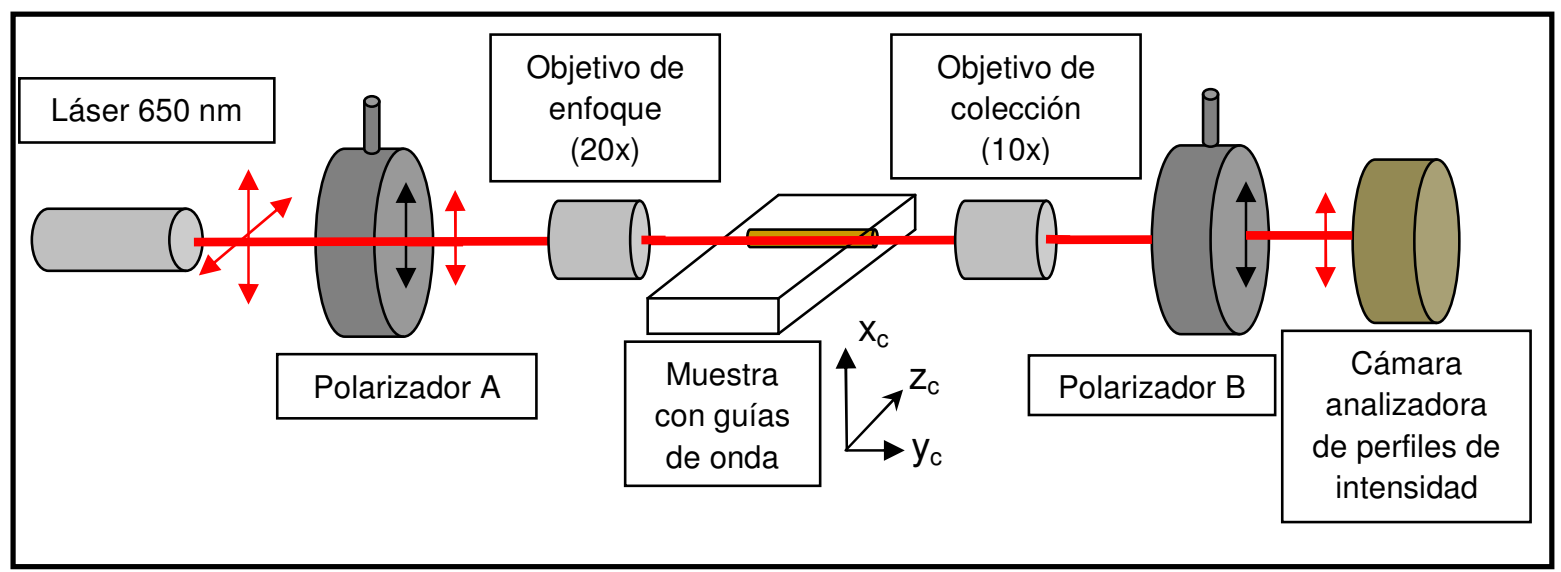

Figura 72. Esquema experimental para la medición de modos guiados en polarización $x_{c}$. Para la polarización $\mathrm{z}_{\mathrm{c}}$ se utilizó una configuración análoga, girando ambos polarizadores $90^{\circ}$

En la Figura 73, se muestran las distribuciones de intensidad guiadas en cada estructura a derecha y a izquierda. En general, la intensidad guiada es aproximadamente simétrica respecto al eje $x_{c}$ para ambas polarizaciones. Se puede observar lo siguiente:

\section{Polarización en eje $x_{c}$}

Con respecto a la intensidad de polarización vertical (componente $x_{c}$ ), se puede afirmar que permite obtener más información acerca de la guía de onda que la polarización horizontal.

En la estructura 1, se puede observar una intensidad guiada de mayor amplitud que en el resto, visualizándose alrededor de cuatro lóbulos no alineados. En la zona cercana a la modificación, el área que ocupa la intensidad es mayor que en las otras estructuras. Además, se pueden observar modos guiados en la zona de filamentación inferior, o segunda modificación. Estos modos "secundarios", también se observan en las modificaciones 2 y 3, pero disminuyendo su intensidad acorde se disminuye la energía utilizada. En estas dos estructuras, se observan modos "principales" practicamente con las mismas características, tres lóbulos relativamente definidos y un cuarto evanescente, 
todos ellos alineados. Esta similitud ocurre debido a que a pesar de que se hicieron con distinta energía, la misma también es absorbida por el material en la zona secundaria en el caso de la estructura 2. En la estructura 4 se observan 3 lóbulos a cada lado cuasialineados, mientras que en la estructura 5 se observan dos lóbulos de cada lado de la misma. Por lo tanto, acorde disminuye la energía de pulso de las guías fabricadas, éstas soportan menor cantidad de modos guiados.

También se puede observar que a medida que aumenta la energía del láser aumenta el grado de asimetría de las guías acoplada.

\section{Polarización en eje $\mathbf{z}_{c}$}

La magnitud de la intensidad guiada para esta polarización es, en general, menor a la de la polarización vertical, para todas las energías de pulso.

Por otro lado, la intensidad de la luz guiada en polarización horizontal, tiene menos diferencia entre las distintas energías que la polarización vertical. En general, la magnitud de la intensidad guiada disminuye cuando la energía utilizada para la fabricación de la guía disminuye, y, la región ocupada por la distribución de intensidades en polarización horizontal $\left(\mathrm{z}_{\mathrm{c}}\right)$ es mayor que aquella que ocupa la distribución de intensidades en polarización vertical $\left(\mathrm{x}_{\mathrm{c}}\right)$. 


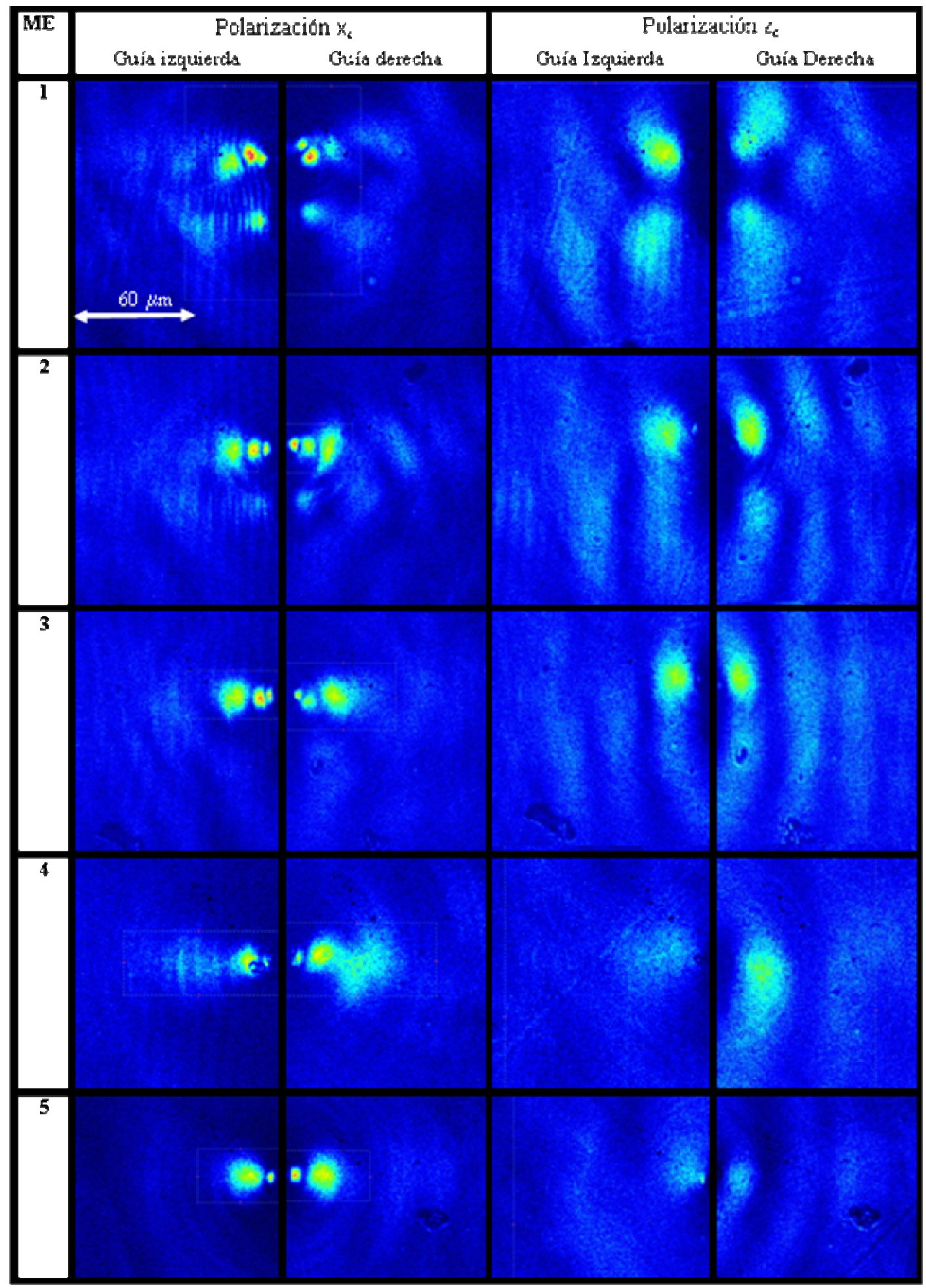

Figura 73. Intensidad guiada de polarización $x_{c}$ y $z_{c}$ en guías generadas a ambos lados de cada modificación estructural (ME). Todas las imágenes están en la misma escala espacial y se obtuvieron bajo las mismas condiciones de filtrado, contraste y procesamiento. La longitud de onda es de $650 \mathrm{~nm}$. 


\section{Comparación de distribución de intensidad experimental con modelo numérico.}

Utilizando el modelo numérico presentado en el Capítulo 3, se evaluaron distintas geometrías y parámetros de expansión $(a, b, \alpha$ y $\beta)$. Después de algunas ejecuciones del mismo, se obtuvieron tres casos numéricos que se pueden asociar a las medidas experimentales (casos 3, 4 y 5). Como el modelo numérico, no tiene en cuenta el fenómeno de filamentación ó modificación secundara, las modificaciones 1 y 2 no son reproducibles por el mismo.

Para los casos 3, 4 y 5, el modelo numérico ajustó de forma aceptable a las medidas experimentales, principalmente para la polarización vertical $\left(x_{c}\right)$. Aunque, en la polarización horizontal $\left(z_{c}\right)$ se observa menor concordancia, se puede ver una disminución de intensidad acorde disminuye la energía de pulso tanto para la medida experimental como para el resultado numérico. En la Figura 74, se presentan estos tres casos y se comparan con las medidas experimentales. Las dimensiones de la elipse utilizados en los tres casos son $a=3 \mu \mathrm{m}$ y $b=4 \mu \mathrm{m}$ y los distintos parámetros de expansión se presentan en la Tabla xi. En la misma, se puede apreciar que la expansión (parámetro $\alpha$ ) asociada a las distintas medidas experimentales, aumenta al aumentar la energía de pulso, algo que es esperable de antemano.

\begin{tabular}{|c|c|c|c|}
\hline $\mathrm{ME}$ & 3 & 4 & 5 \\
\hline $\mathrm{e}_{\mathrm{p}}$ & 0.8 & 0.6 & 0.4 \\
\hline$\alpha$ & 0.008 & 0.005 & 0.003 \\
\hline$\beta$ & 0 & 0 & 0 \\
\hline
\end{tabular}

Tabla xi. Parámetros utilizando en el modelo numérico 
Polarización vertical $\left(\mathbf{x}_{\mathbf{c}}\right)$

Polarización horizontal $\left(\mathbf{z}_{\mathrm{c}}\right)$

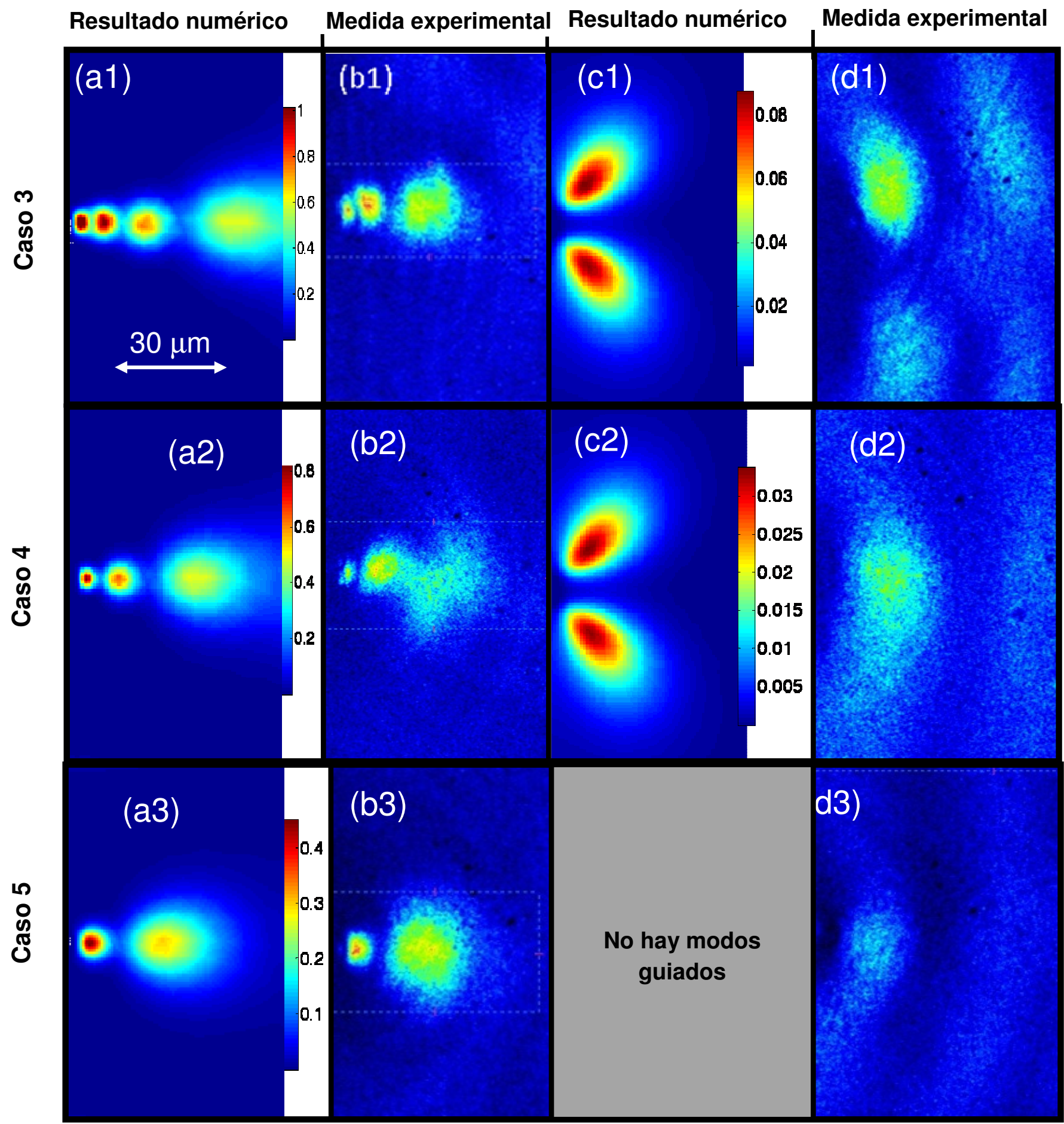

Figura 74. Distribuciones de Intensidades numéricas y experimentales guiadas para ambas polarizciones en los casos 3,4 y 5 . Todas las imágenes tienen la misma escala espacial. 
En la Figura 75, se presenta el campo de índice de refracción asociado a la modificación 3 , obtenido utilizando el modelo numérico. Para los casos 4 y 5 , se obtuvieron análogos campos de índice de refracción pero con los siguientes valores máximos de $\Delta \mathrm{n}_{x}$ y $\Delta \mathrm{n}_{z}$ respectivamente: $1.2 \times 10^{-3}$ y $0.5 \times 10^{-3}$ para el caso 4 y $0.7 \times 10^{-3}$ y $0.1 \times 10^{-3}$ para el caso 5 .

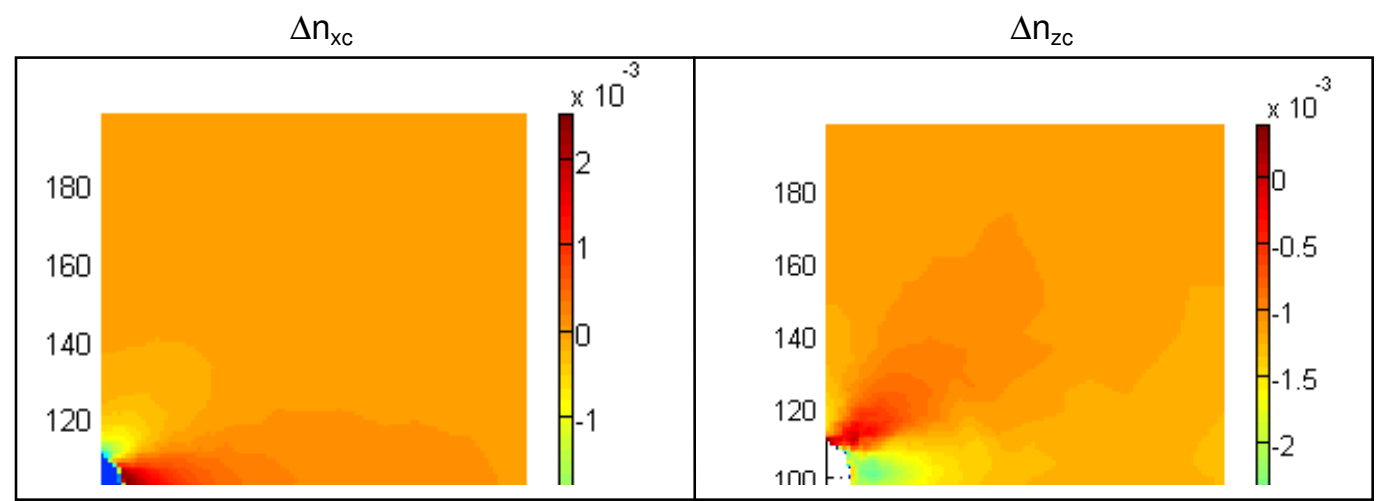

Figura 75. Campos de índice de refracción numérico $\left(\Delta \mathrm{n}_{\mathrm{xc}}\right.$ y $\left.\Delta \mathrm{n}_{\mathrm{zc}}\right)$ para Caso 3

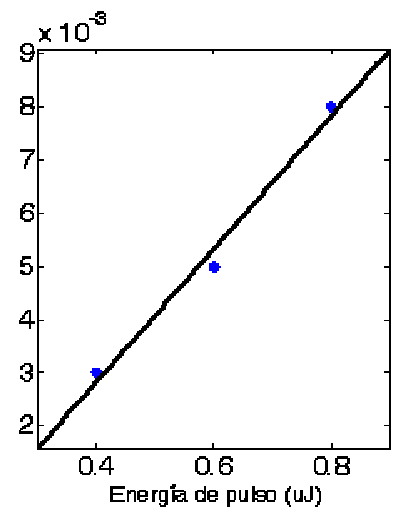

Figura 76. Parámetro de expansión $(\alpha)$ vs energía de pulso $(\mu \mathcal{})$. En círculos se presentan los puntos obtenidos de la comparación y con línea negra el ajuste lineal

Se observa en la Figura 76, que la variación del parámetro de expansión $(\alpha)$ en función de los valores de energía de pulso $\left(e_{p}\right)$, asumiendo las asignaciones efectuadas y para el rango de energía estudiado, se puede aproximar a una recta. La expresión de variación obtenida se presenta en la Ecuación 77.

$$
\alpha=0.012 e_{p}-0.002
$$

En esta sección se estudiaron las polarizaciones guiadas por estructuras generadas con láser de femtosegundos para diferentes valores de energía de pulso. A su vez, se asociaron las medidas experimentales con distintas expansiones del modelo numérico. Se obtuvo, en general, una concordancia razonable entre el modelo numérico y las medidas realizadas. 


\section{Conclusiones y trabajo futuro}

En este trabajo se estudiaron guías de onda Tipo II [BUR07] generadas con láser de femtosegundos fabricadas tanto en niobato de litio dopado con magnesio y neodimio, como en niobato de litio sin dopantes. Debido a que las guías se originan por deformaciones residuales de la interacción, resulta de interés para los análisis $\mu$-Raman propuestos, conocer el comportamiento del espectro Raman del Nd:Mg:LiNbO${ }_{3}$ bajo presión hidrostática. Por esto, se realizó un estudio bajo presión hidrostática hasta 6.3 GPa utilizando el método de celda de diamantes para compresión (DAC) [JAY83]. Este estudio se realizó en colaboración con el Dr. A.Goñi (Universidad Autónoma de Barcelona). Se observó, en rango completo de medición, que los fonones del $\mathrm{Nd}: \mathrm{Mg}: \mathrm{LiNbO}_{3}$ no observan significativas variaciones en el coeficiente de presión respecto del $\mathrm{LiNbO}_{3}$ sin dopantes, a excepción del fonón $\mathrm{E}(\mathrm{TO})_{4}$ que tiene una variación del $400 \%$ y que en apariencia, todos los fonones poseen una relación lineal entre la posición espectral y la presión aplicada.

Este trabajo se publicó en una revista con referato de circulación internacional (1).

Analizando en detalle el comportamiento de los fonones $\mathrm{A}_{1}(\mathrm{TO})$ en función de la presión, se observó que el comportamiento no responde estrictamente a una relación lineal, dependiendo del fonón estudiado. Debido a que el estudio de guías de onda involucra corrimientos espectrales relativamente pequeños de los fonones Raman, se ajustó el comportamiento de estos fonones para un rango reducido de presiones hidrostáticas (hasta 2.5 GPa), obteniéndose coeficientes lineales de presión distintos a los del rango completo de presión. De todas formas, para presiones menores a $0.5 \mathrm{Gpa}$, que es el rango correspondiente a las detectadas en las guías de onda estudiadas, se observa alta dispersión en la posición espectral de los fonones $A_{1}(T O)_{1}$ y $A_{1}(T O)_{4}$.

Debido a que las guías de onda fabricadas con láser de femtosegundos tienen su origen en una expansión mecánica, durante este trabajo se generaron los siguientes modelos numéricos en entorno MATLAB para estudiarlas:

- Modelo elástico: para determinar el campo de deformaciones y su correspondiente variación de índice de refracción generado por una expansión estática y arbitraria, en el seno de un medio elástico.

- Modelo óptico: para determinar la distribución de intensidades guiadas en el campo de índice de refracción generado.

La implementación del modelo elástico en entorno MATLAB dio lugar a un segundo artículo publicado en una revista con referato de circulación internacional (2). Donde, además, se ofreció libremente este código. A raíz de esto, investigadores de distintos países lo solicitaron y se le fue enviado: Dr. Atoosa Arabanian (Irán), Sr. Klaus Bergner (Alemania), Dr. Yang Tan (China) e Ing. Jerome Lapointe (Canadá), entre otros. 
Luego, utilizando estos modelos numéricos y códigos, se realizaron los siguientes análisis:

i) Análisis unidimensional (guías de onda en $\mathrm{Ng:Mg:LiNbO} 3$ ):

Para evaluar, en una primera aproximación, el desempeño del modelo propuesto, los campos de deformaciones obtenidos numéricamente se ajustaron a los corrimientos micro-Raman medidos en una trayectoria lineal que atraviesa una guía de onda fabricada con láser de femtosegundos. Esto se realizó en el marco de la Teoría de Deformación Potencial. La misma, define los corrimientos espectrales, respecto de la posición natural de los fonones Raman como función lineal del estado de deformación de la estructura cristalina.

En este análisis, se utilizaron complementariamente los corrimientos de los fonones Raman bajo presión hidrostática del $\mathrm{Nd}: \mathrm{Mg}: \mathrm{LiNbO}_{3}$ para definir los valores de los parámetros del modelo numérico (geometría y magnitud de expansión) utilizando distintas estrategias.

En la trayectoria unidimensional estudiada, el modelo ajustó de forma adecuada los corrimientos de los fonones Raman medidos experimentalmente. Permitiendo inferir que la expansión mecánica que ocurre en la interacción estudiada sería principalmente horizontal.

Utilizando este análisis unidimensional de las guías fabricadas con láser de femtosegundos, se publicó un tercer artículo en una revista con referato de circulación internacional (3).

Para verificar y contrastar el resultado obtenido en este primer análisis, se realizó un análisis bidimensional.

\section{ii) Análisis bidimensional (guías de onda en $\mathrm{Ng}: \mathrm{Mg}: \mathrm{LiNbO}_{3}$ ):}

Este estudio se llevo a cabo en una nueva estructura fabricada en el Grupo de Óptica (Fotón Charro) de la Universidad de Salamanca. Primeramente, se midieron las distribuciones de intensidades guiadas en las adyacencias de esta estructura. A continuación, se realizó un mapeo bidimensional de los corrimientos de los fonones Raman $\mathrm{A}_{1}(\mathrm{TO})$ en una región que abarca la modificación estructural fabricada y la zona de guiado. Luego, se calcularon los campos de corrimientos Raman bidimensionales utilizando los parámetros óptimos obtenidos del estudio unidimensional, para su comparación con los experimentales. Se observó una importante concordancia entre el cálculo y la medida experimental, fundamentalmente para el fonón $\mathrm{A}_{1} \mathrm{TO}_{4}$. El fonón $A_{1}(T O)_{1}$ tiene menor grado de concordancia y el fonón $A_{1}(T O)_{2}$ aún menor. Para el fonón $\mathrm{A}_{1}(\mathrm{TO})_{3}$ no se detectó señal.

Luego, utilizando el mapa experimental Raman del fonón $\mathrm{A}_{1}(\mathrm{TO})_{4}$ y el modelo numérico generado, se realizaron distintos ajustes para determinar, nuevamente, los parámetros de expansión óptimos. Se observó un ajuste aceptable entre el modelo numérico y las medidas experimentales.

A su vez, los resultados obtenidos se compararon con estudios realizados por otros autores. Cómo principal conclusión, se confirmó que, en las guías estudiadas, la expansión mecánica que ocurre después de la interacción láser-material es 
principalmente horizontal (dirección $z_{c}$ ). Esto, permitió explicar porqué en las adyacencias de las modificaciones, es posible el guiado de luz horizontalmente polarizada, en general, de menor intensidad. A su vez, no se condice a lo afirmado previamente por otros autores, debido a que los mismos no contemplaron la posibilidad de que la expansión mecánica originada en la interacción sea principalmente en la dirección $z_{c}$.

Una posible explicación de esta característica de la expansión, es que la misma se debe a la orientación del cristal. De acuerdo a H. Ledbetter [LED04], el cristal de niobato de litio tiene propiedades de fricción interna sumamente anisotrópicas. En la dirección $x_{c}$ e $y_{c}$, el coeficiente de fricción es cuatro veces mayor que en la dirección z. Por lo tanto, bajo condiciones de carga dinámica, el material ofrece menor resistencia a deformarse en la dirección $z_{\mathrm{c}}$. Para corroborar esto, está previsto fabricar estructuras en cristales de niobato de litio de distinta orientación y realizar estudios $\mu$-Raman y de intensidad guiada.

Por otro lado, se determinaron valores estimativos para las Constantes de Deformación Potencial $\left(e\right.$ y $f$ ) de los fonones $A_{1}(\mathrm{TO})$ del niobato de litio y se compararon (para el fonón $\mathrm{A}_{1}(\mathrm{TO})_{4}$ ) con los obtenidas por otro autor, que utilizó un método distinto, dando valores similares.

Estos análisis dieron lugar a dos trabajos más, publicados en sendas revista internacionales con referato $(\mathbf{4 , 5})$.

Luego, se propuso y aplicó un método directo para determinar los campos de índice de refracción a partir de los mapas de corrimientos Raman medidos y las Constantes de Deformación Potencial Calculadas. Este método tiene la ventaja de que no necesita pasos iterativos, requiere la medición del mapa de corrimiento espectral de dos fonones $\mathrm{A}_{1}(\mathrm{TO})$ y conocer las constantes mencionadas. Con los campos de índice de refracción calculados, se calculó la distribución de intensidad guiada y se comparó con la medida experimental. Se observó un mejor desempeño del método utilizando los fonones $\mathrm{A}_{1}(\mathrm{TO})_{1}$ y $\mathrm{A}_{1}(\mathrm{TO})_{4}$.

iii) Fabricación de guías de onda con láser de femtosegundos en $\mathrm{LiNbO}_{3}$ sin dopantes $\mathrm{y}$ caracterización de polarizaciones guiadas.

Utilizando un láser de femtosegundos disponible en el CIOp, se fabricaron con diferentes energías de pulso, modificaciones estructurales que permiten el guiado de luz en sus adyacencias. En las mismas, se acopló luz láser $(650 \mathrm{~nm})$ utilizando el método End-fire y se utilizó un conjunto de polarizadores para guiar luz de polarización horizontal $\left(x_{c}\right)$ y vertical $\left(z_{c}\right)$. Luego, se utilizó el modelo numérico previamente generado para asociar a cada guía una expansión numérica. Se observó una buena concordancia entre el resultado numérico y el experimental. En base a este resultado, se propuso una relación lineal entre la energía de pulso utilizada para la fabricación de la guía y el parámetro de expansión horizontal utilizado en el modelo.

Finalmente, como anexo al trabajo de Tesis, se presenta la aplicación de mapeos Raman (por intermedio del método directo utilizado para calcular deformaciones) para determinar la relación entre la densidad y el módulo de elasticidad. El módulo de elasticidad fue medido utilizando indentación instrumentada, sobre la superficie del cristal y en dirección paralela a la dirección de guiado de luz. Esta medición se obtuvo a través de una trayectoria recta que atraviesa la zona de modificación estructural y la zona de guiado. La 
variación de densidad en la misma trayectoria se obtuvo de los mapeos Raman. Para vincular la variación de densidad con el módulo de elasticidad, se utilizó la ley exponencial propuesta por Gibson y Aslhey.

En resumen, los principales logros de este Trabajo de Tesis, en orden de importancia, se pueden resumir en los siguientes ítems:

-Se determinaron las características geométricas de la expansión mecánica que ocurre cuando un láser de pulsos ultra-cortos (cientos de femtosegundos) interactúa con cristales de niobato de litio. La dirección de escritura fue en dirección del $x_{c}$ del cristal.

-Se propone una explicación cuantitativa, en términos de la Teoría de Deformación Potencial para explicar el origen de los corrimientos Raman de los fonones $A_{1}(T O)$ en guías de onda fabricadas con láser de femtosegundos. Principalmente, para los fonones $\mathrm{A}_{1}(\mathrm{TO})_{1}$ y $\mathrm{A}_{1}(\mathrm{TO})_{4}$.

-Se obtuvieron valores estimativos de las constantes de deformación potencial para los fonones $\mathrm{A}_{1}(\mathrm{TO})_{1}, \mathrm{~A}_{1}(\mathrm{TO})_{2}$ y $\mathrm{A}_{1}(\mathrm{TO})_{4}$ del niobato de litio dopado con Magnesio y Neodimio.

-Se presentó y aplicó un método directo para medir campos de variación de índice de refracción en guías de onda generadas por deformaciones, utilizando mapeos $\mu$-Raman. Este método no requiere iteraciones y se basa en la utilización de mapas de corrimiento espectral de dos fonones $A_{1}(T O)$ y las Constantes de Deformación Potencial de estos fonones, para resolver en cada punto del mapa, el par de ecuaciones lineales que brinda la Teoría de Deformación Potencial.

-Se estudió el guiado de luz en polarización horizontal $\left(z_{c}\right)$ y vertical $\left(x_{c}\right)$ en diferentes estructuras fabricadas con láser de femtosegundos de distintos valores de energía de pulso. Se contrastaron los resultados experimentales con aquellos obtenidos utilizando el modelo numérico, para determinar una relación entre el parámetro de expansión horizontal del modelo y la energía de pulso utilizada para fabricar la guía de onda.

-Se determinó la variación de la posición espectral de los fonones Raman en función de la presión hidrostática (hasta $6 \mathrm{Gpa}$ ) para el niobato de litio dopado con neodimio y magnesio ( $\mathrm{Nd}: \mathrm{Mg}: \mathrm{LiNbO}_{3}$ ).

En base al trabajo realizado, en el futuro, se pretende realizar las siguientes tareas (los tópicos se presentan en orden de prioridad):

a) Efectuar estudios análogos a los efectuados en niobato de litio, pero en otras orientaciones del mismo cristal y en otros materiales de aplicación en fotónica, como ser: Cuarzo $\left(\mathrm{SiO}_{2}\right)$, Silicio $\left(\mathrm{SiO}_{2}\right)$, LiSaF y YLF. El objetivo consiste en evaluar las características de la expansión mecánica que ocurre a partir de la interacción y compararla con la obtenida en el presente trabajo.

b) Utilizar los métodos generados y aplicados en el presente Trabajo de Tesis para estudiar micro-estructuras embebidas en Silicio (y otros materiales semiconductores) de aplicación en electrónica[MA13,RA01,SVE11]. Por ejemplo, en las adyacencias de conductores micrométricos de cobre u oro. El objetivo consiste en medir 
micro-deformaciones residuales de dichas estructuras y sus adyacencias [RYU12]. Esta información tiene potencial aplicación en el diseño de circuitos micro-electrónicos.

c) Estudiar la relación entre las Constantes de Deformación Potencial (e y f) y el coeficiente de presión hidrostático $(\mathrm{d} \omega / \mathrm{dp})$ para deformaciones y presiones relativamente bajas (hasta $0.5 \mathrm{Gpa}$ ) para niobato de litio y otros cristales como Cuarzo $\left(\mathrm{SiO}_{2}\right)$ y $\mathrm{LiSaF}$.

d) Generar un modelo numérico que tenga en cuenta el comportamiento dinámico de la expansión producida por la interacción entre el láser de femtosegundos y cristales. En particular, para el $\mathrm{LiNbO}_{3}$, que tiene una fuerte asimetría en los coeficientes de fricción interna [LED04].

En el marco de esta tesis, se realizaron las siguientes publicaciones:

1) M.R. TEJERINA; K. PEREIRA DA SILVA; A. R. GOÑI; G. A. TORCHIA, Hydrostatic-pressure dependence of Raman-active optical phonons in Nd:Mg:LiNbO 3 , OPTICAL MATERIALS; Lugar: Amsterdam; Año: 2013 p. $1-4$

2) M.R. TEJERINA; G. A. TORCHIA, MATFESA: strain and refractive index field estimation after femtosecond laser interaction with transparent material APPLIED PHYSICS. A - MATERIALS SCIENCE AND PROCESSING; Lugar: Berlin; Año: 2012 vol. 110 p. 591 - 594

3) M.R.TEJERINA; D.JAQUE; G. A. TORCHIA, $\mu$-Raman spectroscopy characterization of LiNbO3 femtosecond laser written waveguides. JOURNAL OF APPLIED PHYSICS; Lugar: New York; Año: 2012 vol. 112 p. $123108-123108$

4) M.R. TEJERINA; D.JAQUE; G. A. TORCHIA, A 2D $\mu$-Raman analysis of low repetition rate femtowaveguides in lithium niobate by using a finite element model, OPTICAL MATERIALS; Lugar: Amsterdam; Año: 2014 vol. 5 p. 936 - 940 .

5) M.R. TEJERINA; G. A. TORCHIA, Computation of the expansion parameters of femto-waveguides using a two dimensional micro-Raman map and guided modes, JOURNAL OF APPLIED PHYSICS; Lugar: New York; Año: 2013 vol. 4 p. 153106 - 153112. 



\section{Anexo A: Exploración de guías de onda fabricadas con láser fs utilizando Indentación Instrumentada y $\mu$-Raman}

En este anexo se presenta un estudio de la variación de módulo de elasticidad y densidad en la zona cercana a un par de modificaciones estructurales generadas con láser de femtosegunos. Se utiliza la Teoría de Deformación Potencial para determinar la variación de densidad en forma directa y la misma se ajusta, mediante la conocida ley de Gibson y Ashley, a la variación del módulo de elasticidad obtenida empleando la técnica de indentación instrumentada. De esta forma, se muestra una aplicación adicional de las Constantes de Deformación Potencial Raman, utilizándolas para estudiar la variación del módulo de elasticidad en función de la densidad del material.

\section{Técnica instrumental}

Para realizar indentaciones en las guías fabricadas en niobato de litio se utilizó un equipamiento comercial de indentación instrumentada Tribolndenter-Hysitron. El mismo se muestra en la Figura 77. Este sistema, permite realizar indentaciones/penetraciones controlando desplazamiento ó carga y midiendo ambas variables simultáneamente en los más diversos materiales con sus superficies adecuadamente preparadas. A su vez, dispone de un sistema automatizado que permite realizar varias indentaciones en distintas ubicaciones de la muestra en forma automatizada.

Este equipo permite emplear puntas de diamantes de diferente geometría diseñadas especialmente para tal fin: piramidal de 3 lados ("Barkovich"), piramidal de 4 lados ("Vickers"), cono-esférica de diferentes diámetros y cilíndrica plana, entre otras. Para el estudio realizado en el presente trabajo se utilizó una punta cono-esférica de $2 \mu \mathrm{m}$ de diámetro.

Las principales características del sistema utilizado son las siguientes:

- Resolución de desplazamiento vertical (Z): $3.1 \mathrm{~nm}$

- Desplazamiento máximo vertical: $5 \mu \mathrm{m}$

- Resolución para carga: $100 \mathrm{nN}$

- Carga máxima: $10 \mathrm{mN}$ 
- Punta utilizada: Cono-esférica de $2 \mu \mathrm{m}$ de radio

- Resolución de desplazamiento lateral (X e Y): $0.5 \mu \mathrm{m}$

- Microscopio incorporado: se utilizó un objetivo Olympus de 10x.

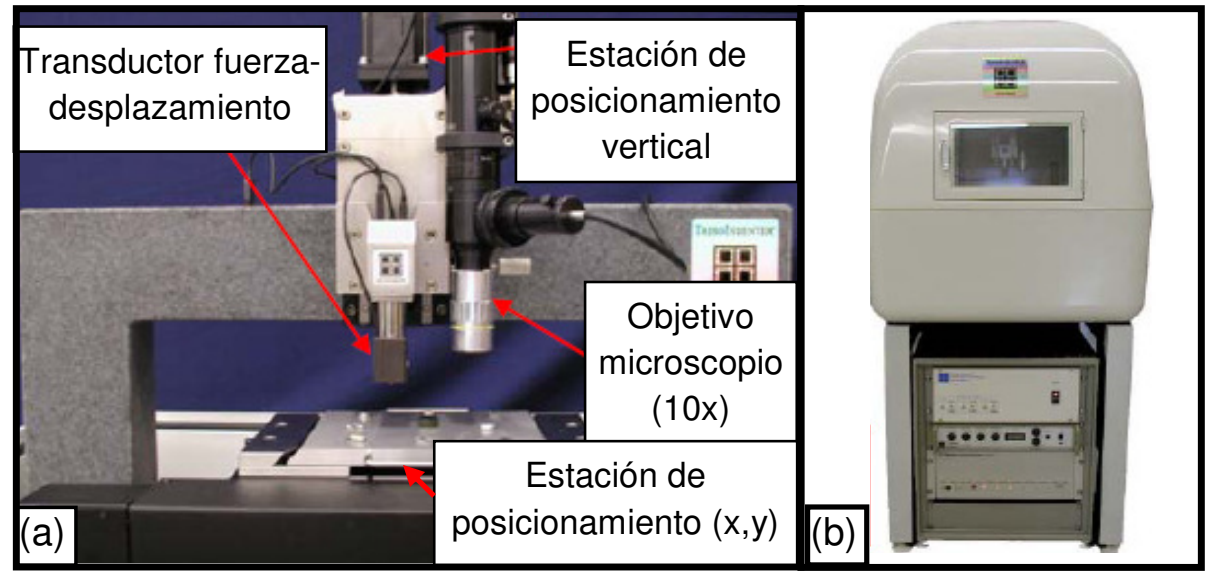

Figura 77(a) Imagen de sistema de posicionamiento y transductor fuerza desplazamiento (b) Imagen del equipo de nano-indentación.

El componente fundamental de este sistema es el transductor fuerza-desplazamiento que permite controlar desplazamientos de algunos nanómetros y cargas de $100 \mathrm{nN}$. Este transductor funciona utilizando tres placas paralelas conductoras (Figura 78). Dos placas fijas y una móvil solidaria al penetrador. Se aplican voltajes a las placas fijas de forma tal que el potencial sea cero en el centro y máximo en estas. Por lo tanto, el voltaje generado en la placa móvil se puede calibrar en función de la distancia. De esta forma, se mide la posición de la placa móvil. Para desplazar la placa central se aplica un voltaje entre esta y la lámina inferior que es proporcional a la fuerza ejercida. Realizando una calibración el sistema determina la fuerza ejercida en función del voltaje aplicado.

Con estos principios, mediante un sistema de control de lazo cerrado se pueden medir y controlar ambas variables: carga y desplazamiento.

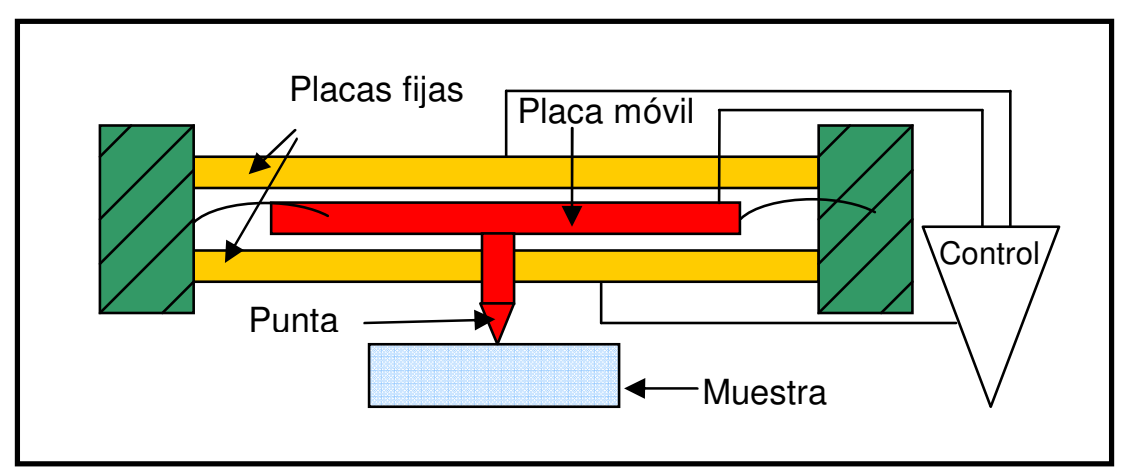

Figura 78. Esquema de transductor fuerza-desplazamiento: placa fija, placa móvil y muestra. 
Una curva típica de carga vs desplazamiento que se puede obtener en dióxido de silicio se presenta en la Figura 79. Los materiales cerámicos tienen en general buenas cualidades para ser estudiados por esta técnica, produciendo curvas de carga vs desplazamiento estables, con alta relación señal-ruido y sin grandes discontinuidades. Por esta razón, se utiliza una muestra de dióxido de silicio para calibrar el equipo antes de cada ciclo de indentaciones.

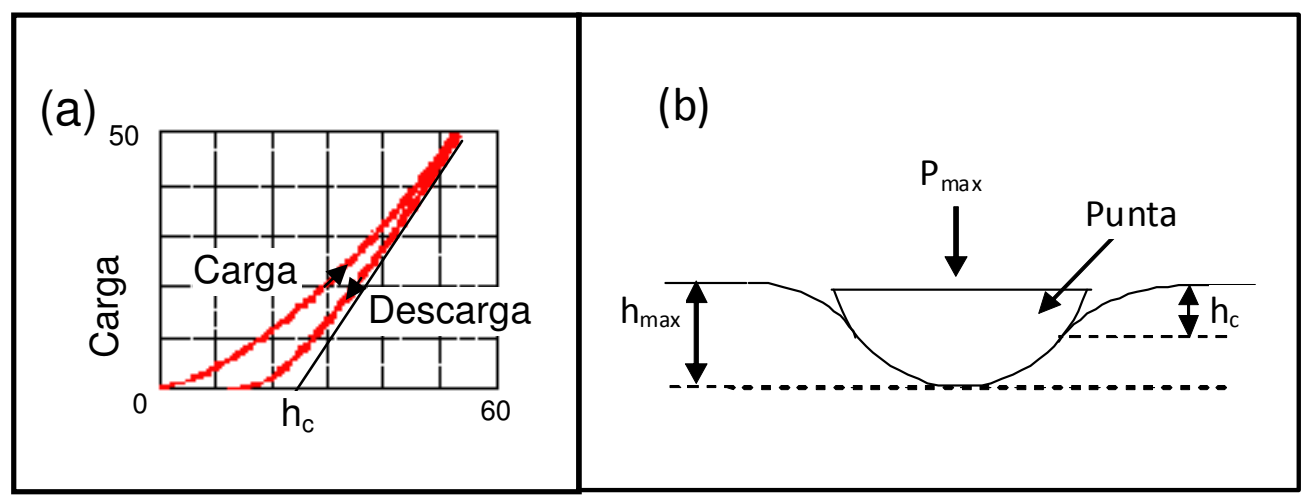

Figura 79. (a) Curva típica de carga vs desplazamiento en muestra de dióxido de silicio, (b) esquema de indentación.

De acuerdo a estudios principalmente de Hertz y Oliver-Pharr sobre mecánica de contacto [OLI04, HE82], la rigidez de contacto $S$ del material está definida por la pendiente inicial de descarga (Ecuación 78). El área de contacto está relacionada con $h_{c}$ por una función que depende de la geometría del indentador (Ecuación 79) y $h_{c}$ se obtiene de la curva de descarga Figura 79(a) (Ecuación 80). A su vez, A y $S$ están asociadas a la constante de elasticidad lineal reducida $E_{r}$ del material por la Ecuación 81. En este trabajo, se utiliza el módulo de elasticidad reducido obtenido por el mismo sistema de pos-procesado del equipo de nano-indentación, que utiliza el método de Oliver-Pharr para obtener $E_{r}$.

$$
S=\left.\frac{d P}{d h}\right|_{P \max }
$$

$A=f\left(h_{c}\right)$

$h_{c}=h_{\max }-h_{s}=h_{\max }-\varepsilon \frac{P_{\max }}{S}$

$E_{r}=\frac{1 \sqrt{\pi} S}{\beta_{g} 2 \sqrt{A}}$ 
En estas ecuaciones: $E_{r}$ es el módulo reducido de elasticidad [OLI04], $\beta_{g}$ es una constante que depende de la geometría del indentador, $\mathrm{A}$ es el área de contacto entre el indentador y la muestra, $\mathrm{P}$ es la carga aplicada, $h_{\max }$ es la profundidad máxima de penetración y $h_{\mathrm{s}}$ la profundidad.

\section{Procedimiento y resultados}

- Las modificaciones estructurales analizadas fueron fabricadas con láser de femtosegundos en $\mathrm{Nd}: \mathrm{Mg}_{\mathrm{LiNbO}} \mathrm{Na}_{3}$ a $90 \mathrm{~m}$ de profundidad utilizando una energía de $1 \mu \mathrm{J}$ y pulsos de $120 \mathrm{fs}$. La muestra se trasladó en dirección $\mathrm{y}_{\mathrm{c}}$ a una velocidad de $30 \mu \mathrm{m} / \mathrm{s}$. El láser de femtosegundos se enfocó con un objetivo de 10x ( $N A=0.25)$. Una imagen de las mismas se presenta en la Figura 80 (d)

- Se realizaron indentaciones a carga máxima $(1000 \mu \mathrm{N})$ de a intervalos de $1.5 \mu \mathrm{m}$, a través de las modificaciones generadas con el láser de femtosegundos, siguiendo la trayectoria $b_{1} b_{2}$ (Figura $80(d)$ ). La dirección de aplicación fue coincidente con el eje $y_{c}$.

- Se realizó un mapa $\mu$-Raman de la región lindante a las modificaciones mostradas en la Figura 80(d). La configuración Raman utilizada es de retro-dispersión ("back-scattering") Y(ZZ)Y, obteniéndose el característico espectro (Sección 5.2) para cada punto. La resolución espacial del mapeo fue de $1 \mu \mathrm{m}$ tanto en el eje $z_{c}$ como en el eje $x_{c}$. Se ajustaron los fonones $A_{1}(T O)_{1}, A_{1}(T O)_{2}$ y $A_{1}(T O)_{4}$ para el espectro correspondiente a cada punto del mapa utilizando el software LabSpec $\circledast$ y se obtuvieron los corrimientos en número de onda $\left(\mathrm{cm}^{-1}\right)$ respecto a su posición característica, mostrados en la Figura 80. 


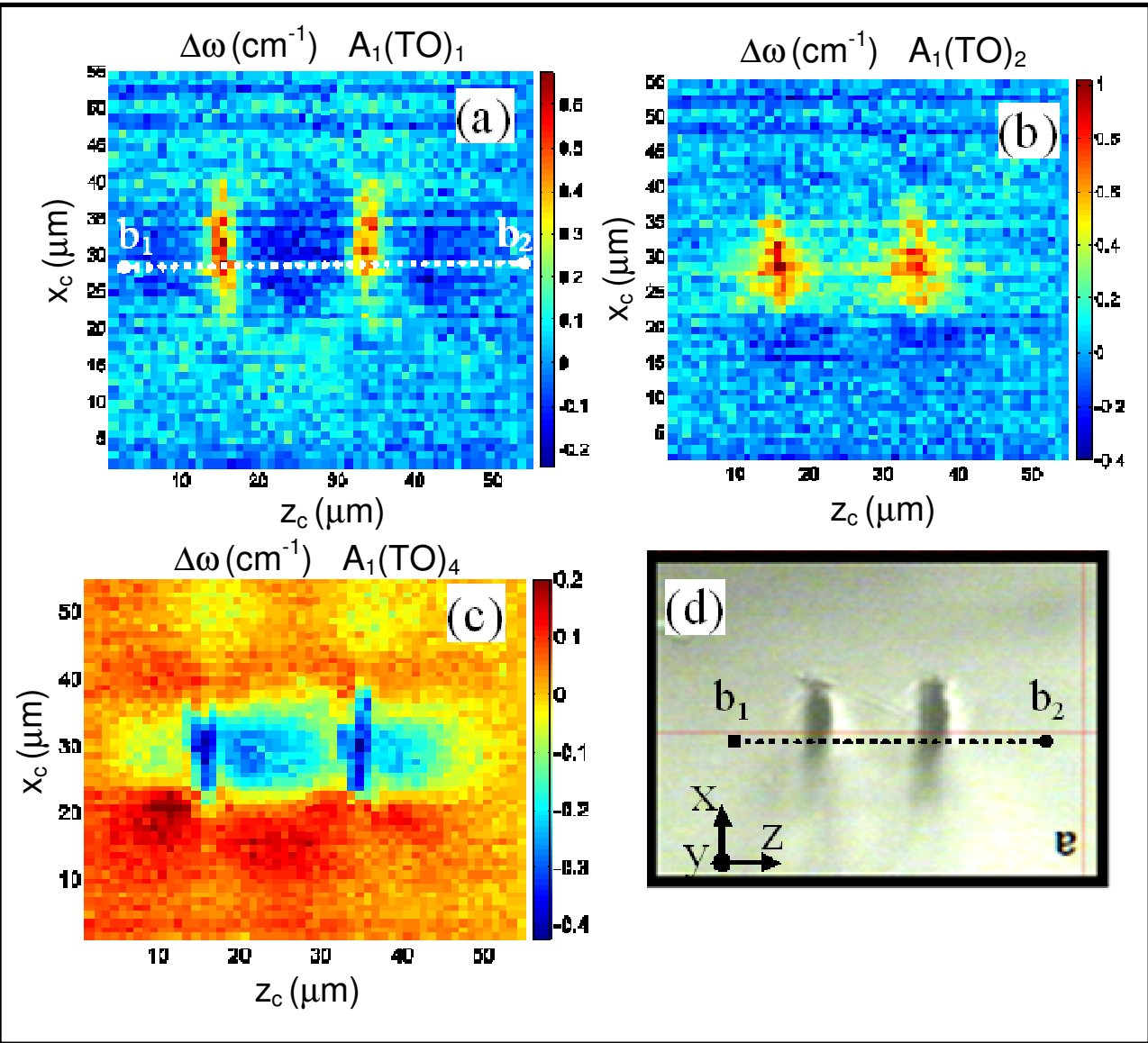

Figura 80. (a),(b) y (c) Mapas $\mu$-Raman, (d) imagen de modificaciones estructurales. La separación entre ambas es de $20 \mu \mathrm{m}$, los ejes corresponden a la estructura cristalina.

De acuerdo a lo analizado en la Sección 5.3, para estimar la variación de volumen en la zona de interacción directa y en los alrededores es conveniente, en el cálculo directo, utilizar la combinación de los fonones $A_{1}(T O)_{1}$ y $A_{1}(T O)_{4}$. La metodología para obtener de forma directa el campo de deformaciones también se explica en dicha sección. Utilizando la combinación de fonones mencionada, y definiendo la variación de volumen como $\Delta \mathrm{V} / \mathrm{V}=\varepsilon_{\mathrm{x}}+\varepsilon_{\mathrm{z}}$ [BUR08] se obtuvo el mapa de variación de volumen presentado en la Figura 81. Se puede ver que el volumen relativo obtenido en la zona de interacción directa se incrementa en la zona central en aproximadamente $1 \times 10^{-2}$, y que esta variación es relativamente abrupta en la zona central. 


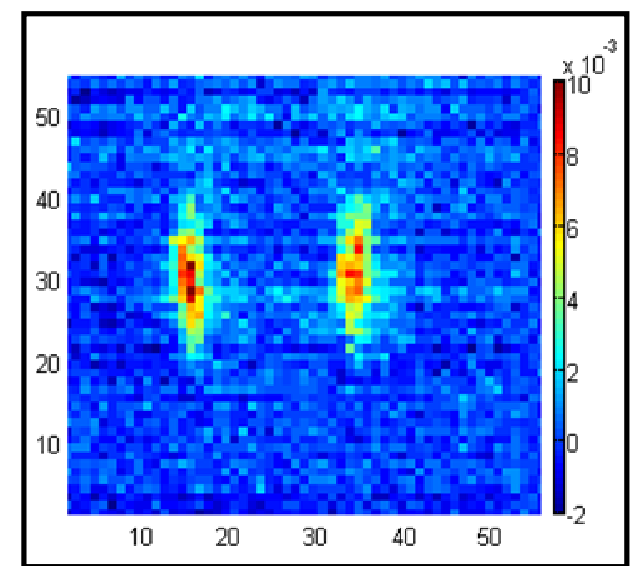

Figura 81.Variación de volumen

estimada utilizando $\mu$-Raman

Derivando la expresión de la densidad $(M / V)$ respecto a $V$, dividiendo ambos términos por $\rho_{o}$ y despejando $\rho / \rho_{o}$ se obtiene la Ecuación 82.

$$
\frac{\rho}{\rho_{o}}=1-\frac{\Delta V}{V}
$$

La relación entre el módulo de elasticidad y la variación de densidad se expresa, de acuerdo a la ley de Gibson y Ashley [GIB82] mediante la Ecuación 83.

$$
\frac{E}{E_{o}}=\left(\frac{\rho}{\rho_{o}}\right)^{b^{E}}
$$

Aplicando logaritmo decimal a ambos miembros de la Ecuación 83, el exponente $b^{E}$ está representado por la pendiente de la recta que ajusta los valores $\log (E / E O)$ vs $\log (r / r o)$. En la Figura 82, se puede ver que los puntos, aunque con una importante dispersión, tienen una relación lineal. Por consiguiente, se presenta un ajuste lineal de estos datos. La pendiente de la recta ajustada es $b^{E}=9 \pm 2$. La dispersión de estos datos se puede asociar, principalmente, al ruido obtenido en el mapeo del módulo de elasticidad (Figura 83(b), puntos azules). 


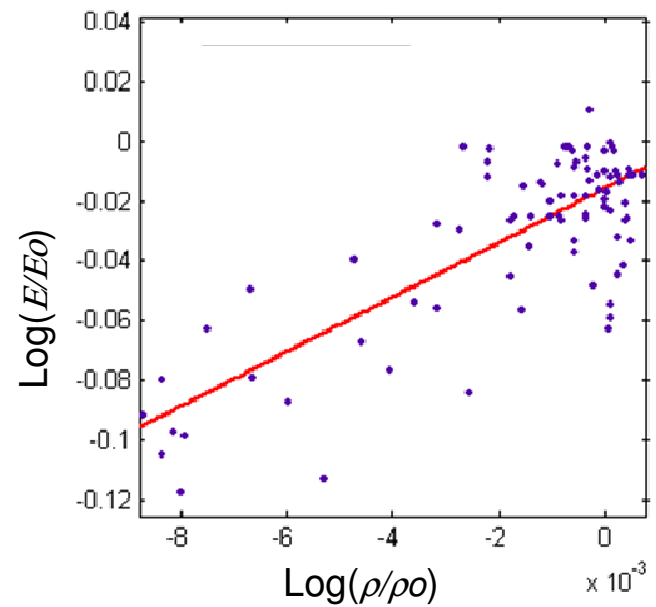

Figura 82. $\log \left(E / E_{o}\right)$ vs $\log \left(\rho / \rho_{0}\right)$ representado por puntos, y su ajuste lineal representado por la curva roja.

En la Figura 83(a), se presenta una imagen de la región estudiada en la que se pueden observar las improntas de las indentaciones en la trayectoria CD. En la Figura 83(b) se observa el módulo de elasticidad relativo medido en la misma trayectoria (círculos azules) y el ajuste realizado con la ley presentada en la Ecuación 83 y la variación de densidad relativa obtenida de los corrimientos Raman (línea roja). Se observa un ajuste aceptable entre ambos datos (los mismos se interpolaron para realizar el ajuste numérico porque fueron medidos con diferentes intervalos). 


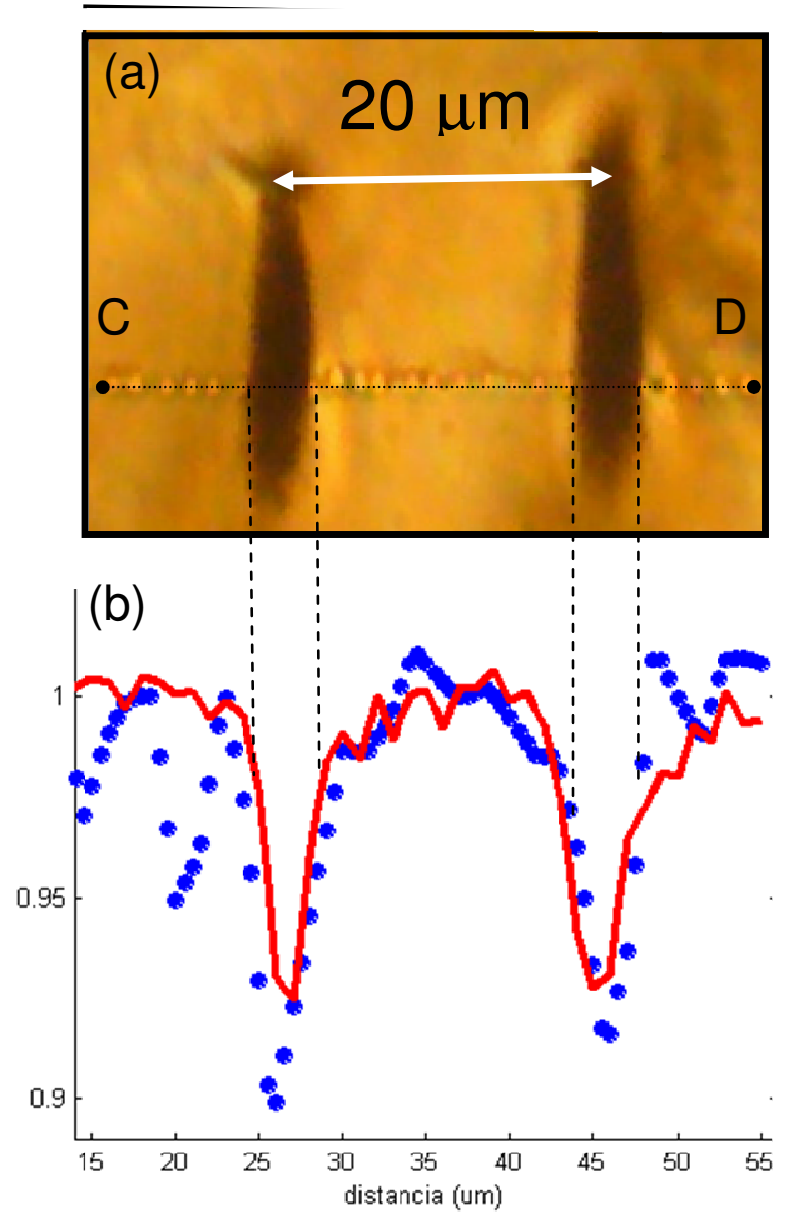

Figura 83. (a) Imagen de modificaciones estructurales e improntas. (b) $E / E_{o}$ (puntos azules) y $\left(\rho / \rho_{0}\right)^{9}$ (curva roja). 


\section{Anexo B: Códigos generados y/ó utilizados para aplicar el Método de Elementos Finitos en entorno MATLAB}

En este Anexo se presentan los principales códigos generados y/ó utilizados en entorno MATLAB en este trabajo de tesis y se detalla la autoría de los mismos.

Índice de códigos

\section{$\boldsymbol{A}$}

ajustestrainx.m

C

campoindtri2.m

192

codigol.m

172

codigo $2 . \mathrm{m}$

189

codigo $3 . \mathrm{m}$

194

coordenadaspath.m

184

D

dcircle.m

186

delipse.m

186

difs.m

177

distmesh2d.m

187

drectangle.m

$\boldsymbol{F}$

fajuste.m

feaplyc2.m

feasmbl1.m

federiv2.m

feeldof.m

feglqd1.m

feglqd2 .

feisog9.m

fejacob2.m

fekine2d.m

fematiso.m 


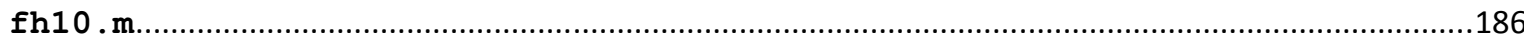

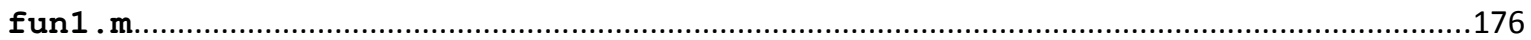

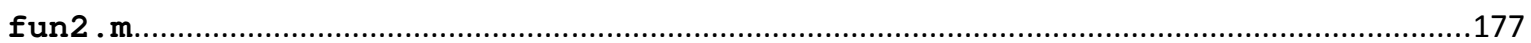

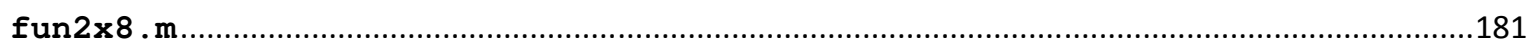

M

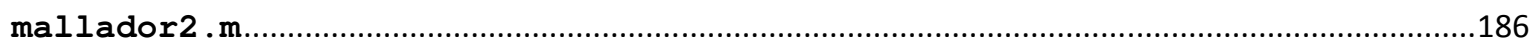

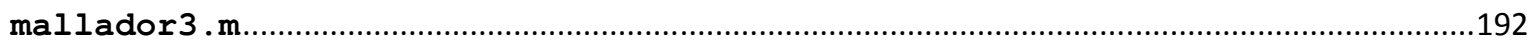

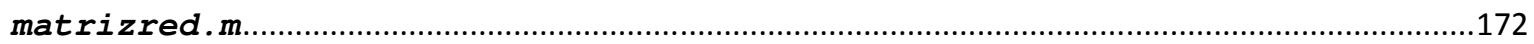

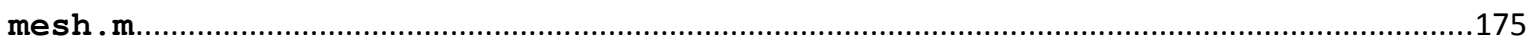

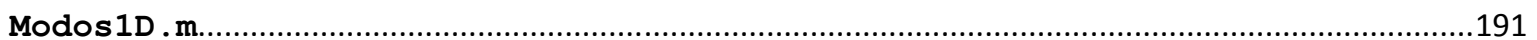

$\boldsymbol{R}$

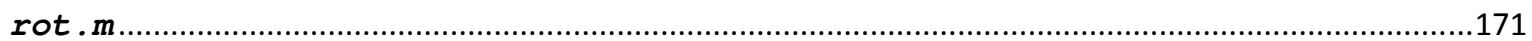

$S$

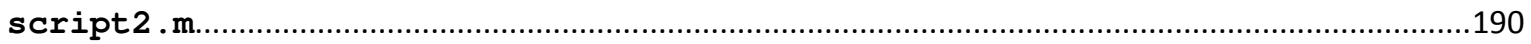

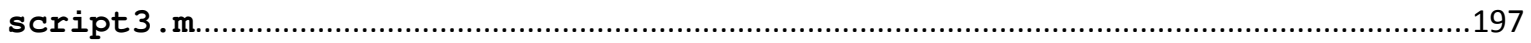

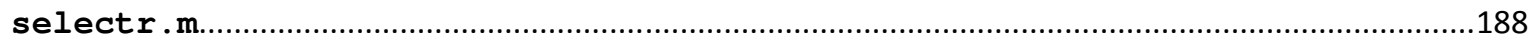

\section{Funciones del modelo elástico}

En las Figuras 84, 85, 86 y 87 se presentan las jerarquías y descripciones resumidas de las funciones implementadas para los distintos códigos. Con la sigla G.P. se denotan las funciones de Generación Propia, con la letra K, aquellas Generadas por Kwon [KWON97], y con la letra $P$ aquellos generados por Perrson [PER04]. En el texto de los códigos se describen los parámetros de entrada y objetivo específico de cada función. Para obtener mayor detalle de los códigos que no sean de generación propia se debe recurrir a las fuentes mencionadas.

\begin{tabular}{|c|c|c|c|}
\hline $\begin{array}{c}\text { Rotación de } \\
\text { matriz } \\
\text { constitutiva }\end{array}$ & rot.m GP & $\begin{array}{c}\text { Cálculo de matriz } \\
\text { constitutiva reducida } \\
\text { para deformaciones } \\
\text { planas }\end{array}$ & matrizred.m GP \\
\hline
\end{tabular}

Figura 84. Funciones asociadas a la matriz constitutiva del material 


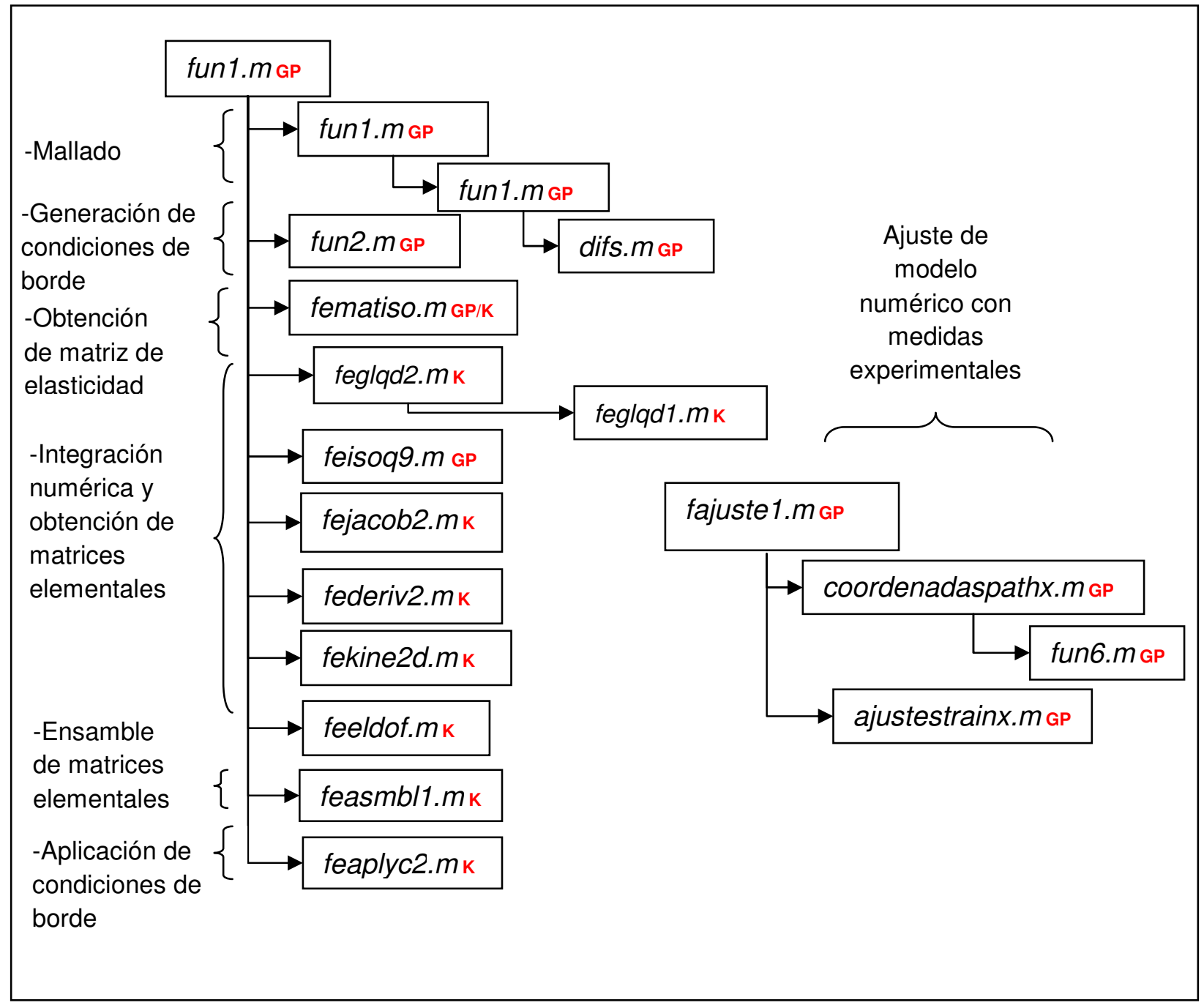

Figura 85.Subfunciones de Codigo1.m y fajuste1.m 


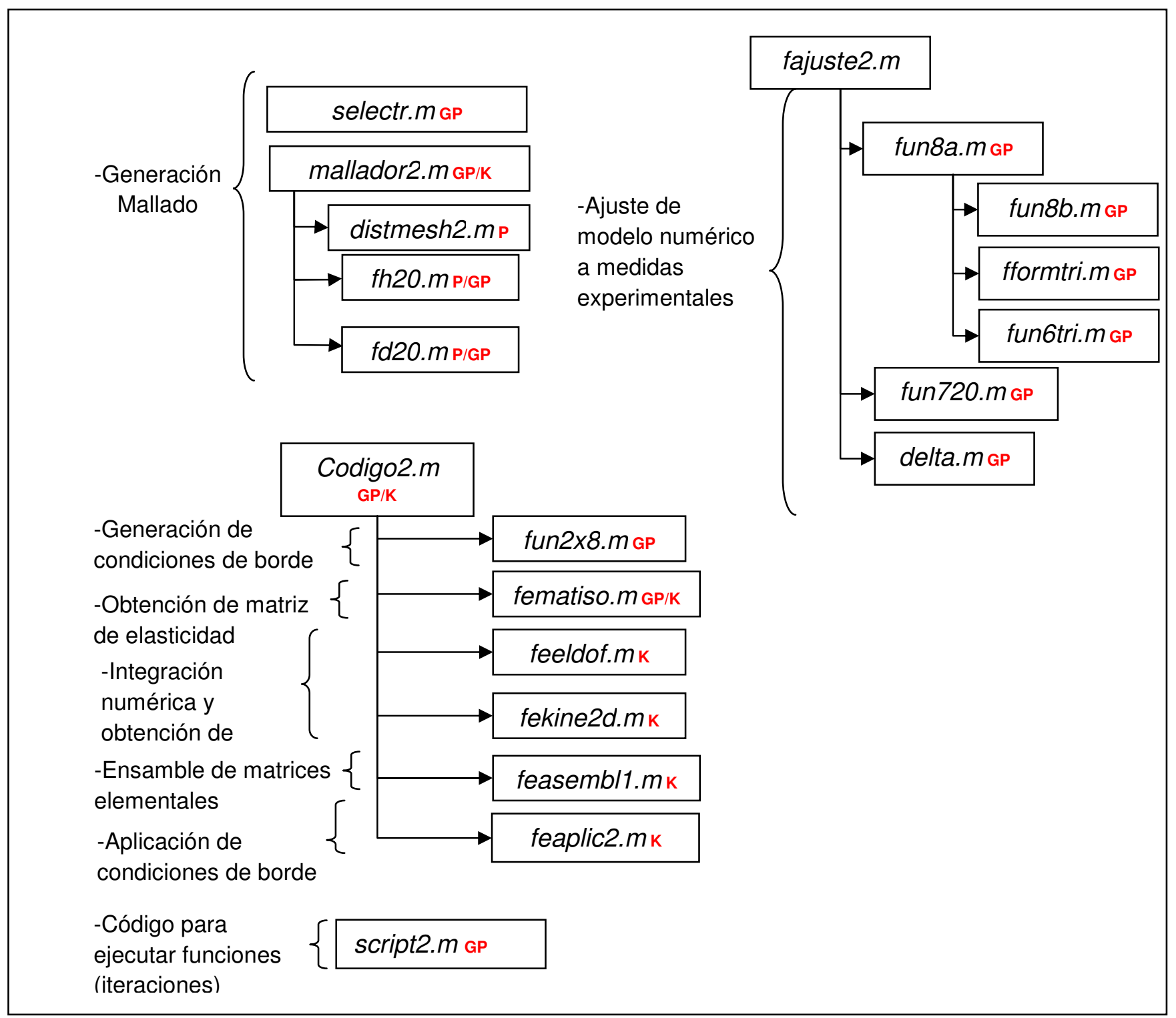

Figura 86. Funciones de mallador2.m, codigo2.m y fajuste2.m 


\section{Funciones del modelo óptico:}

\begin{tabular}{ll|}
$\begin{array}{l}\text {-Calculo de modos } \\
\text { para guía plana } \\
\text { (unidimensional) }\end{array}$ & $\left\{\begin{array}{l}\text { modos 1D.m GP/K } \\
\text {-Mallado de dominio } \\
2 \mathrm{D}\end{array}\right.$ \\
-Aplicación de relación \\
piezo-óptica \\
mallador3.m $m$ K/GP \\
-Cálculo de modos \\
guiados \\
-Código para ejecutar \\
funciones
\end{tabular}

Figura 87. Funciones del modelo óptico

\section{Funciones utilizadas:}

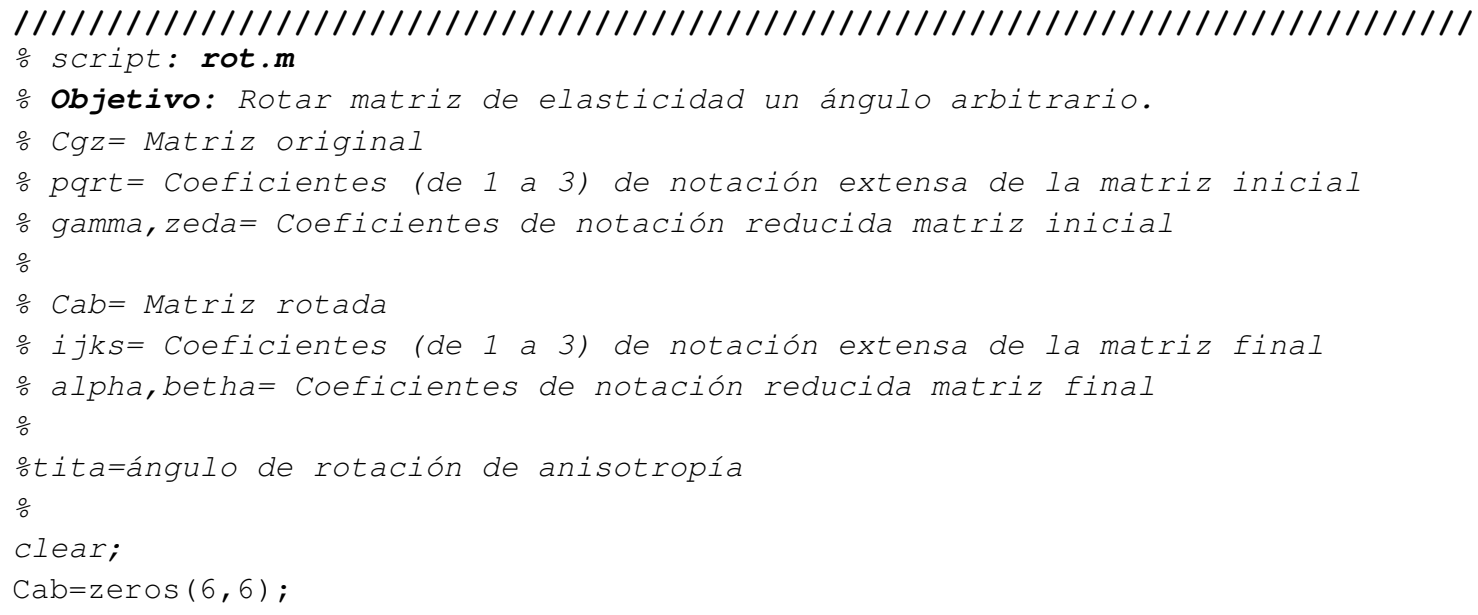




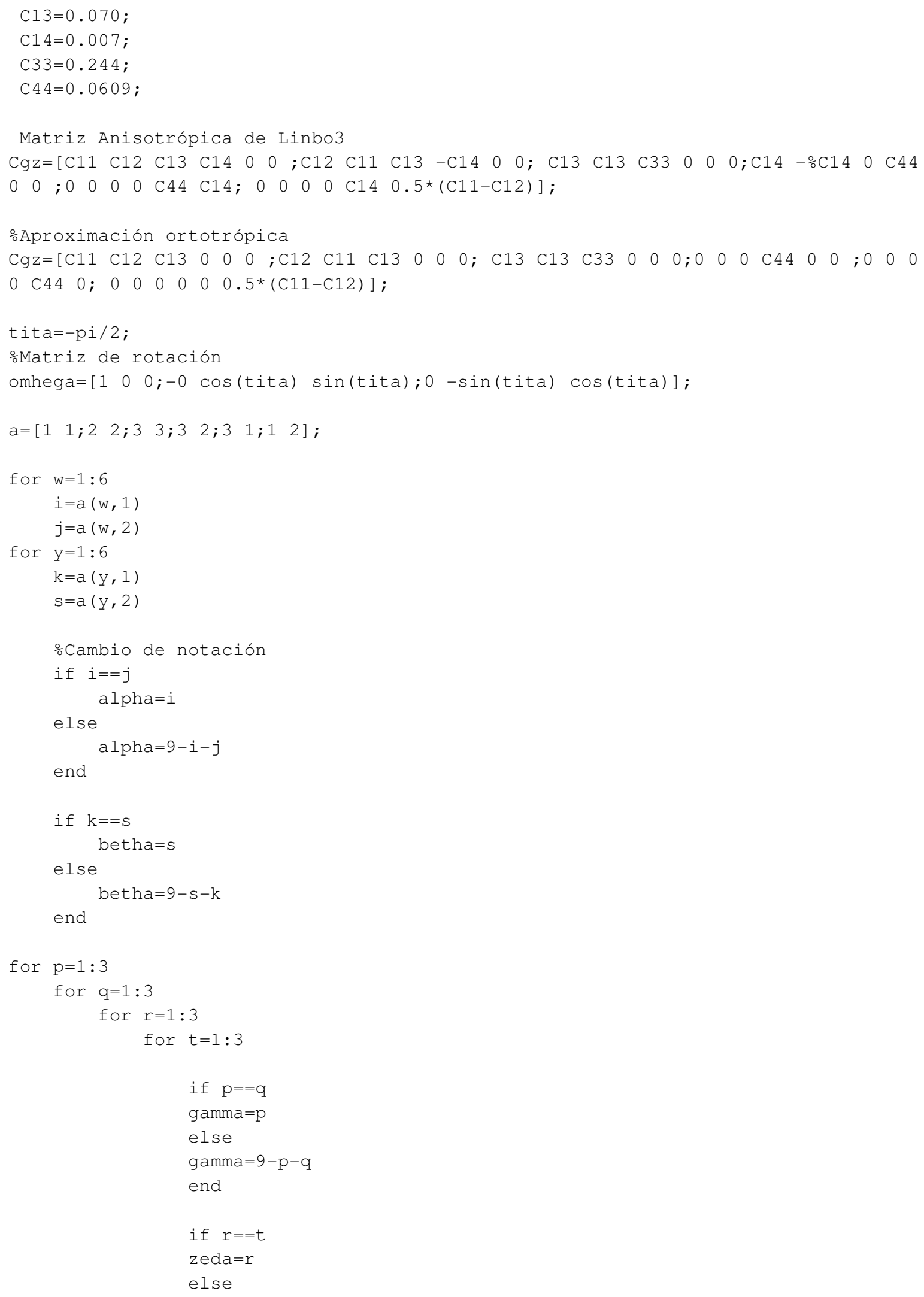




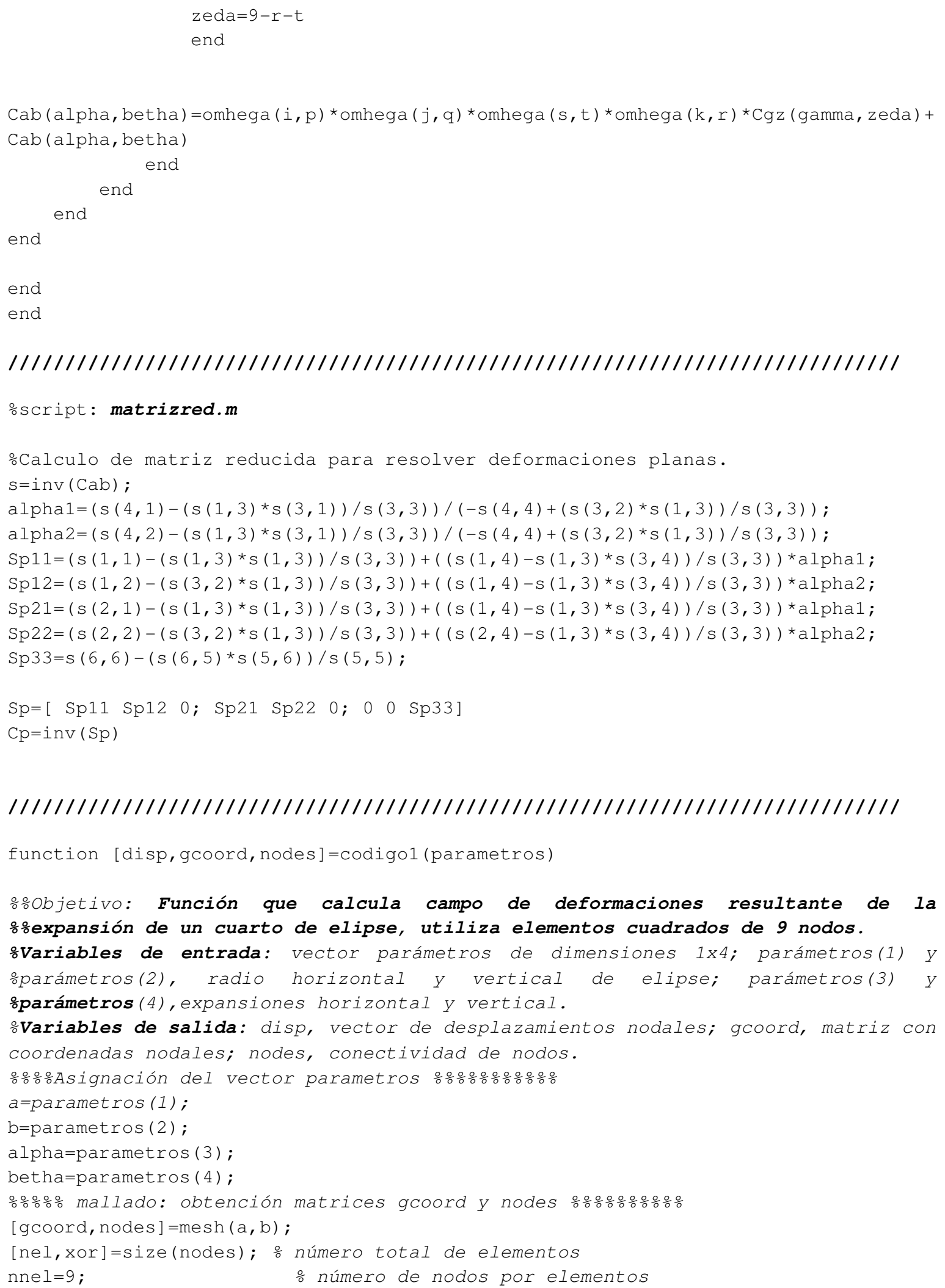


ndof=2; $\quad \frac{o}{0}$ número de grados de libertad por nodos

nnode=length (gcoord); onúmero total de nodos en el sistema

sdof=nnode*ndof; $\quad$ onúmero total de total system dofs

edof=nnel*ndof; $\quad$ odegrees of freedom per element

emodule=0.2; $\%$ módulo de elasticidad[se utiliza solo para material isótropo]

poisson=0.3; \%ódulo de Poisson[se utiliza solo para material isótropo]

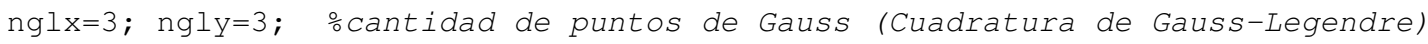

$\mathrm{nglxy}=\mathrm{nglx}{ }^{\star} \mathrm{ngly}$; $\frac{\circ}{\mathrm{n} u m e r o}$ de puntos de Gauss por elemento

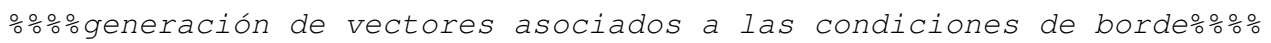

[bcdof, bcval] =fun2 ( gcoord, nodes, a, b, alpha, betha) ;

응응 inicialización de matrices y vectores $\because \frac{0}{0} \% \frac{0}{0} \% \circ \% \%$

ffezeros (sdof, 1$)$; $\quad \frac{\circ}{0}$ vector global de fuerzas

kk=zeros (sdof, sdof); $\quad$ matriz global $K$

disp=zeros (sdof,1); $\quad$ ovetor de desplazamientos del sistema

eldisp=zeros(edof,1); $\frac{\circ}{\circ}$ vector de desplazamientos de elemento

stress=zeros (nglxy, 3); \% matriz elemental de tensiones

strain=zeros (nglxy,3); \% matriz elemental de deformaciones

index=zeros (edof,1); $\quad$ o vector de índices de elemento

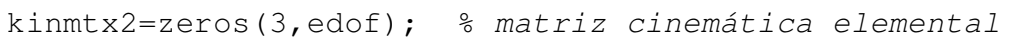

matmt $x=$ zeros $(3,3) ; \quad$ omatriz constitutiva

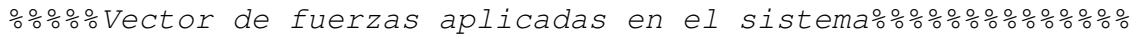

ff $(1)=0$;

응응응응응응응 Cómputo de matrices elementales y su ensamble응응응응응응응응응응응응응응응응응응응

[point2, weight2]=feglqd2(nglx,ngly); $\circ$ puntos de Gauss y pesos para integración onumérica.

matmtx=fematiso(4,emodule,poisson); ㅇmputo de matriz constitutiva

for iel=1:nel $\quad$ iteración para el total de elementos

for $i=1$ :nnel o iteración para cada nodo del elemento

nd $(i)=$ nodes (iel,i); $\frac{\circ}{0}$ coordenada del elemento iel

xcoord $(i)=g c o o r d(n d(i), 1)$; oxtrae el valor $x$ del nodo

ycoord $(i)=g c o o r d(n d(i), 2)$; oxtrae el valor $y$ del nodo

end

k=zeros(edof, edof); $\frac{\circ}{0}$ Inicialización de matriz elemental

응 $\frac{0}{0} \% \circ \% \%$

for intx=1:nglx

$x=$ point $2($ int $x, 1)$;

wt $x=$ weight $2(\operatorname{int} x, 1)$;

for inty=1:ngly

$y=$ point $2($ inty, 2$)$;

wty=weight $2($ inty, 2$)$;

\% puntos de gauss en el eje $x$

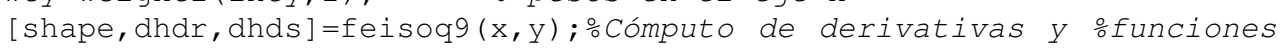
de forma en los puntos de gauss.

jacob2=fejacob2 (nnel, dhdr, dhds, xcoord, ycoord);

detjacob=det (jacob2); $\quad$ o determinante del Jacobiano

invjacob=inv(jacob2); $\quad \frac{\circ}{0}$ inverso del Jacobiano

응응 Cálculo de derivativas de funciones de forma응응응응응응응응

[dhdx, dhdy] =federiv2 (nnel, dhdr, dhds, invjacob) ;

kinmtx2=fekine2d(nnel, dhdx, dhdy); \%cómputo de matriz cinemática

응응응응으의 to matriz elemental응응응응응응응응응응응응

$\mathrm{k}=\mathrm{k}+\mathrm{kinmtx} 2^{\prime}{ }^{*}$ matmt $\mathrm{x} * \mathrm{kinmtx} 2{ }^{*} \mathrm{wt} \mathrm{x}$ *wty ${ }^{*}$ det jacob;

end

end

Fin de iteración de integración numerica

응응응 extraer grados de libertad asociados al elemnento iel 응응응응응응응응응

index=feeldof (nd, nnel, ndof);

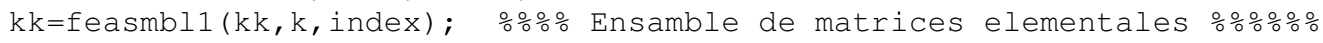

end

응응 Aplicación de condiciones de borde응응응 
$[\mathrm{kk}, \mathrm{ff}]=\mathrm{feaplyc} 2(\mathrm{kk}, \mathrm{ff}, \mathrm{bcdof}, \mathrm{bcval})$;

응으을

disp=kk\ff;

end

//////////////////////////////////////////////////////////////////

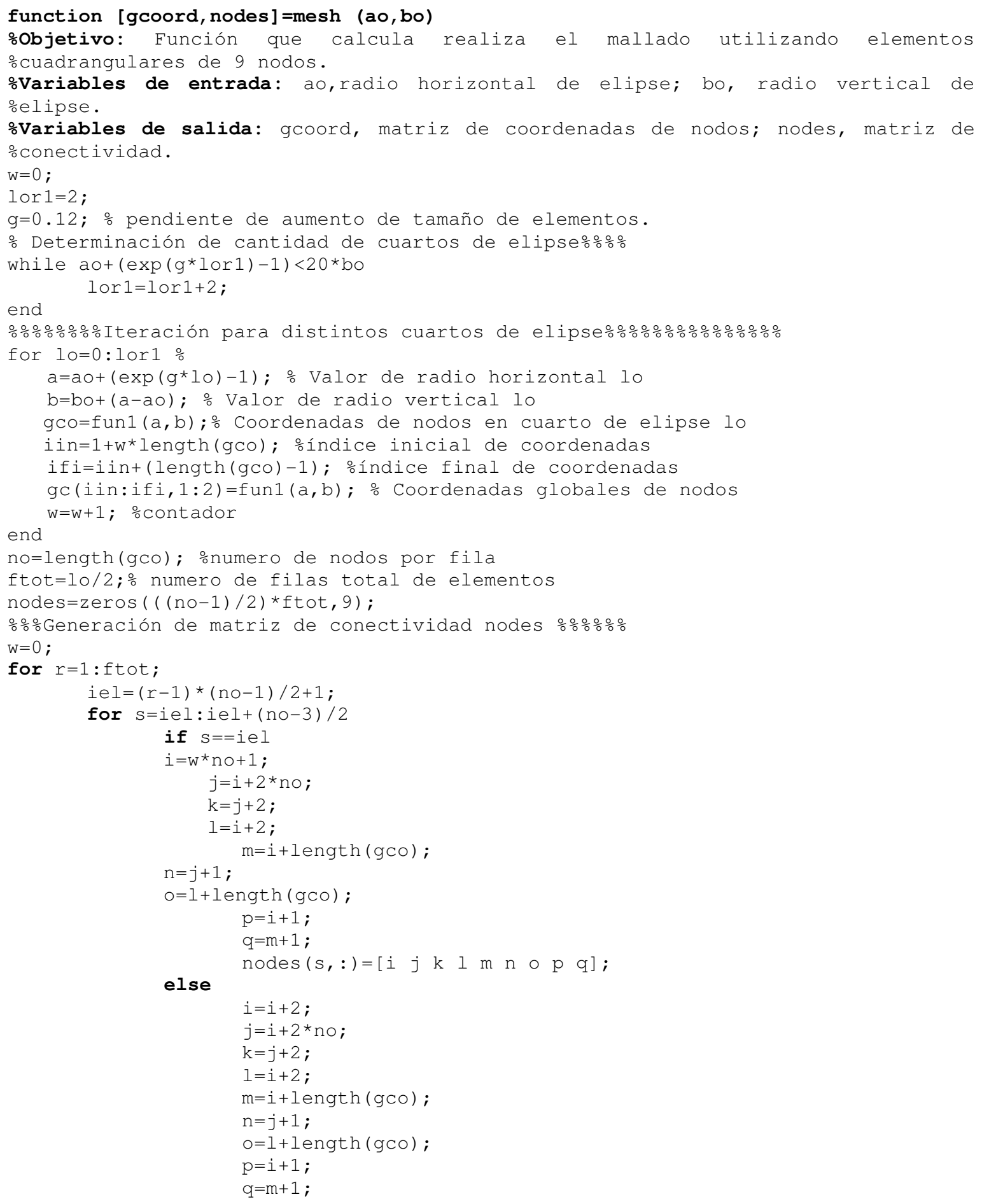




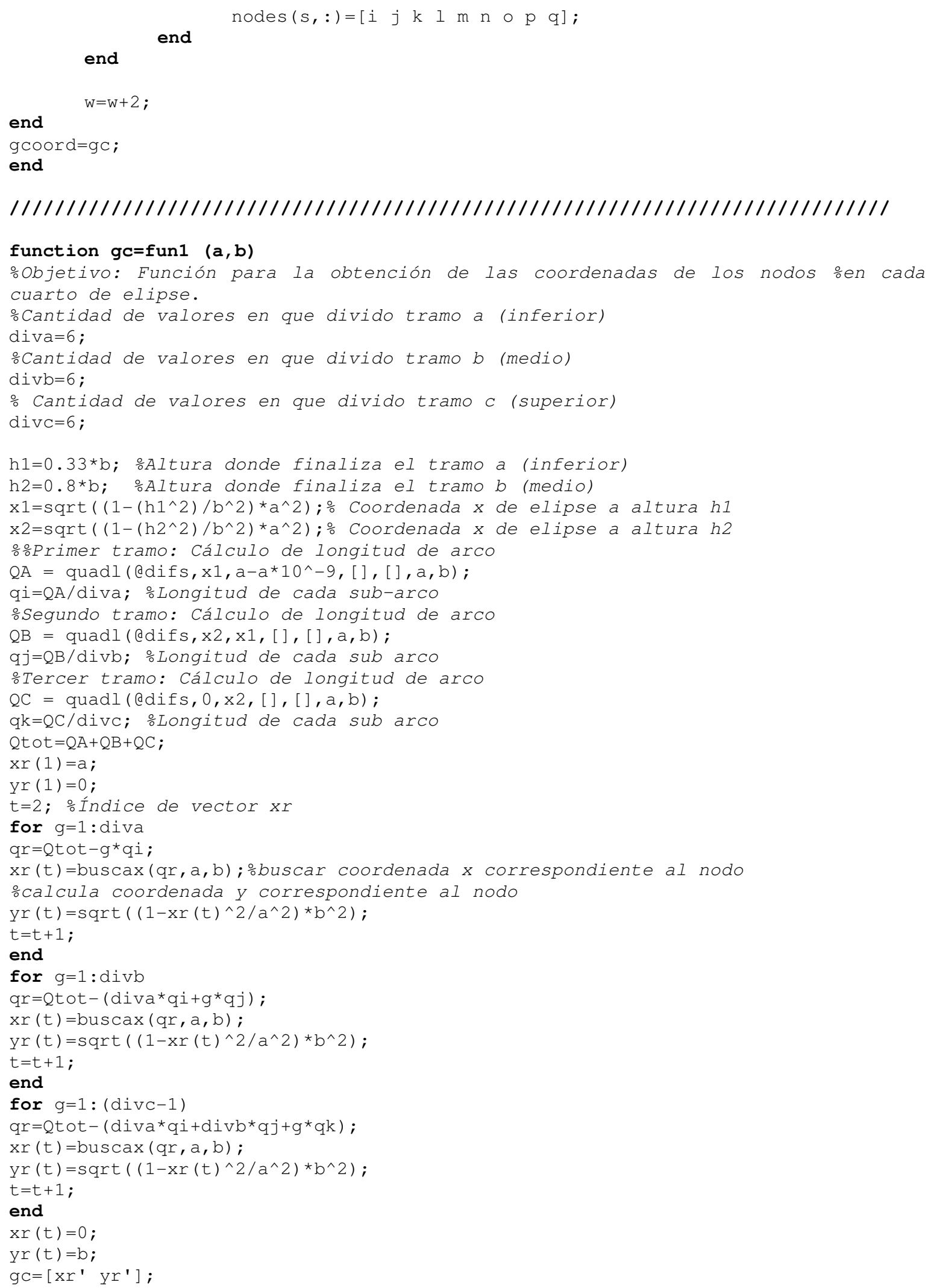


function ds=difs $(x, a, b)$

objetivo:Función para obtener el diferencial de perímetro de una elipse \% $\% \frac{0}{0} \%$ \%Variables de entrada:x, coordenada $x$ del punto donde calcula el diferencial; $\frac{\circ}{2}$, radio horizontal de elipse; b, radio vertical de elipse.

oVariable de salida:ds, diferencial de perímetro, en la coordenada $x$. yprima $=-x^{\star} b^{\wedge} 2 \cdot /\left(\left(\left(1-x \cdot{ }^{\wedge} 2 / a^{\wedge} 2\right) * b^{\wedge} 2\right) \cdot \wedge 0 \cdot 5^{*} a^{\wedge} 2\right) ;$

ds=sqrt $(1+($ yprima $) \cdot \wedge 2)$;

end

$/ / / / / / / / / / / / / / / / / / / / / / / / / / / / / / / / / / / / / / / / / / / / / / / / / / / / / / / / / / / / / /$

function [bcdof, bcval] =fun2 (gcoord, nodes, a, b, alpha, betha)

objetivo:Función que calcula las restricciones de simetría y desplazamientos oiniciales

oVariables de entrada: gcoord y nodes, matrices de mallado; a y b, radios ohorizontal y vertical; alpha y betha, parámetros de expansión horizontal y overtical.

$\mathrm{w}=1$;

for $i=1$ : length (gcoord)

if $\operatorname{gcoord}(i, 2)<1 e-4 \&(((\operatorname{gcoord}(i, 1) / a) \wedge 2+(\operatorname{gcoord}(i, 2) / b) \wedge 2<0.99999) \mid$

$\left.\left(\left((\operatorname{gcoord}(i, 1) / a)^{\wedge} 2+(\operatorname{gcoord}(i, 2) / b)^{\wedge} 2\right)>1.00001\right)\right)$ bcdof $(w)=2 * i$;

$\operatorname{bcval}(w)=0$; $\mathrm{w}=\mathrm{w}+1$;

elseif $\operatorname{gcoord}(i, 1)<0.0001 \&\left((\operatorname{gcoord}(i, 1) / a)^{\wedge} 2+(\operatorname{gcoord}(i, 2) / b)^{\wedge} 2<0.99999\right) \quad$ $\left.\left(\left((\operatorname{gcoord}(i, 1) / \mathrm{a})^{\wedge} 2+(\operatorname{gcoord}(i, 2) / \mathrm{b})^{\wedge} 2\right)>1.00001\right)\right)$ bcdof $(w)=2 * i-1$

end

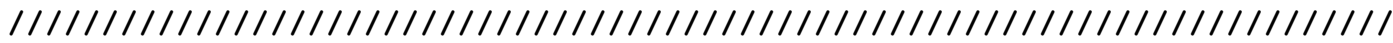

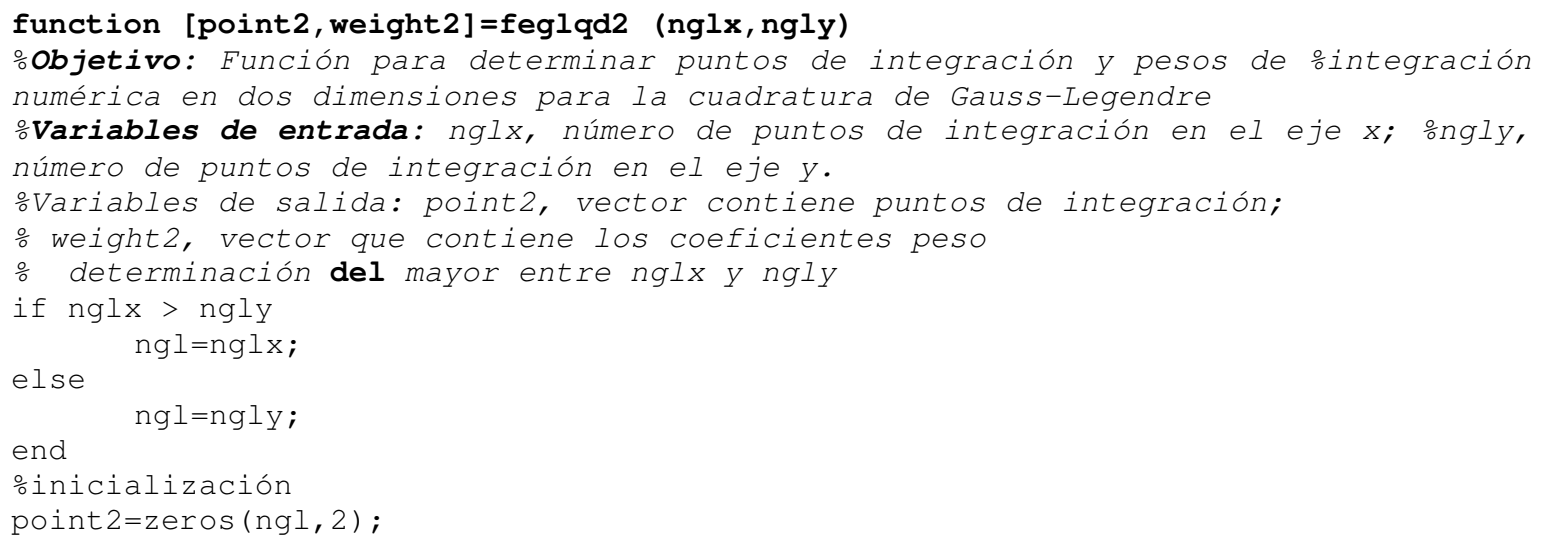




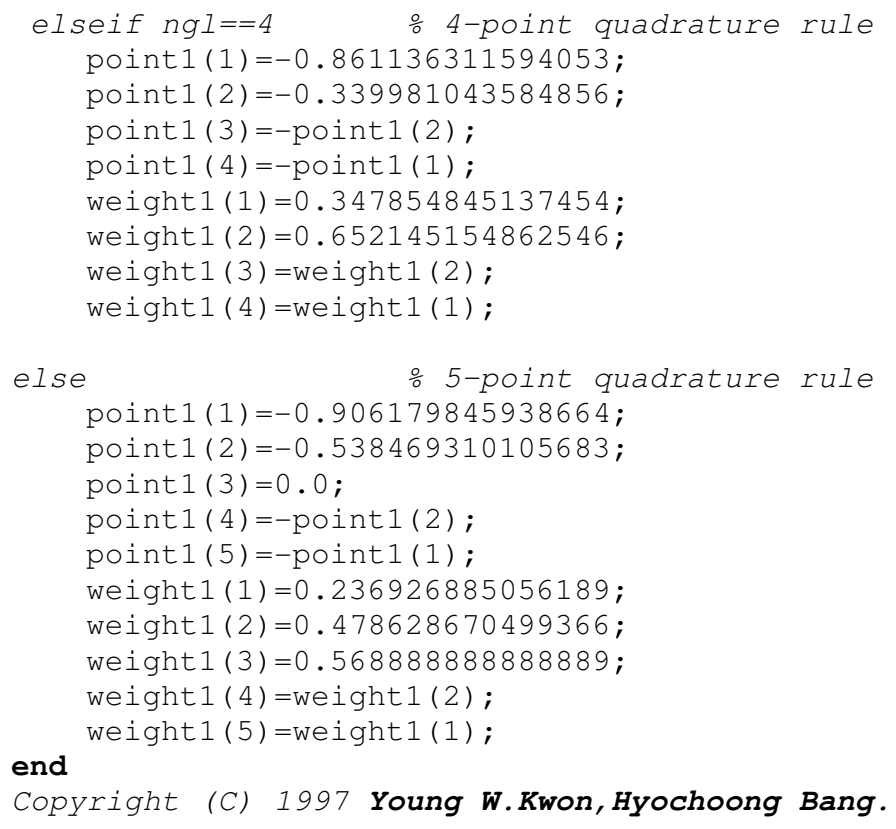

\section{function [matmtrx] =fematiso (iopt, elastic, poisson)}

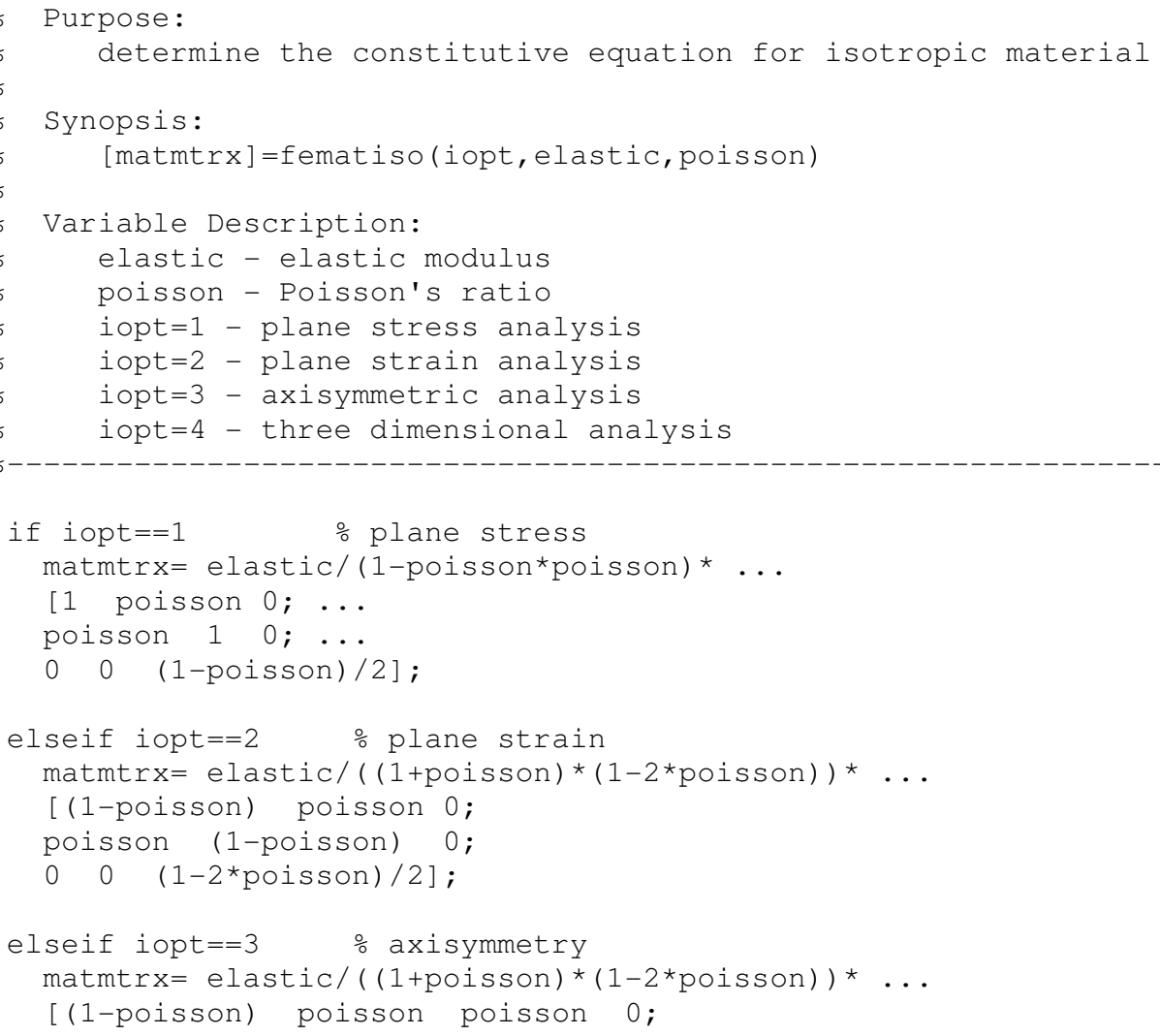




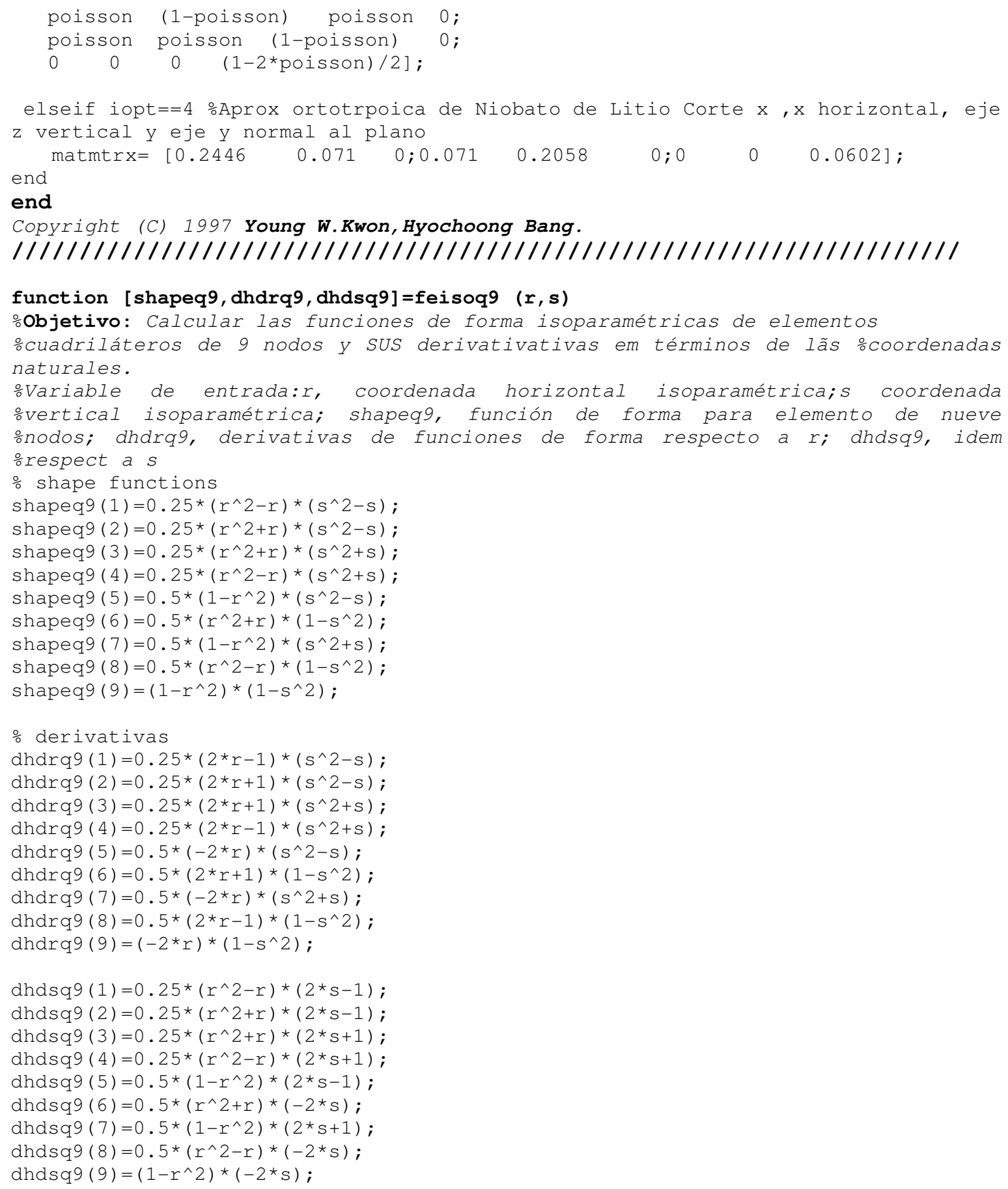




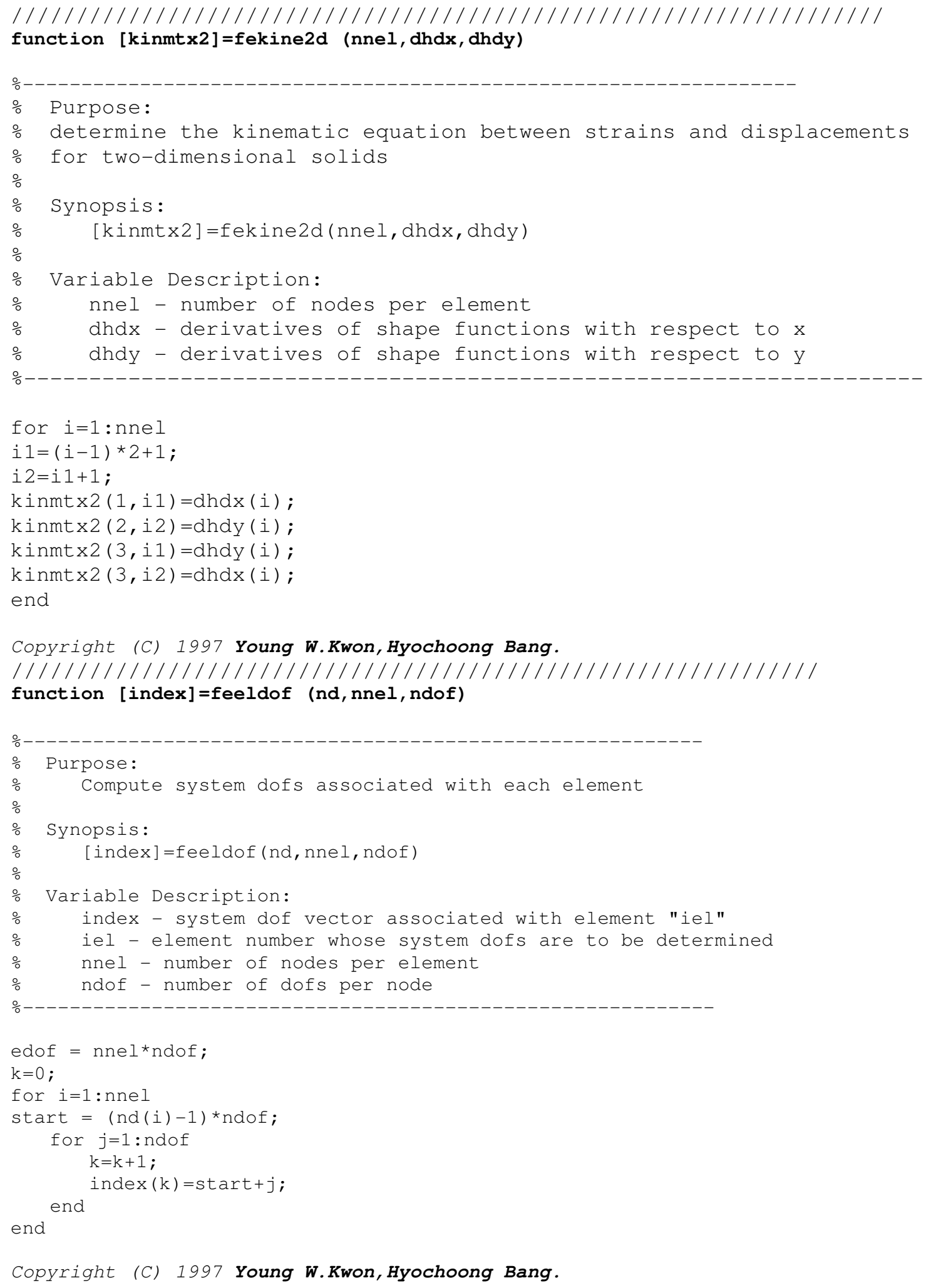




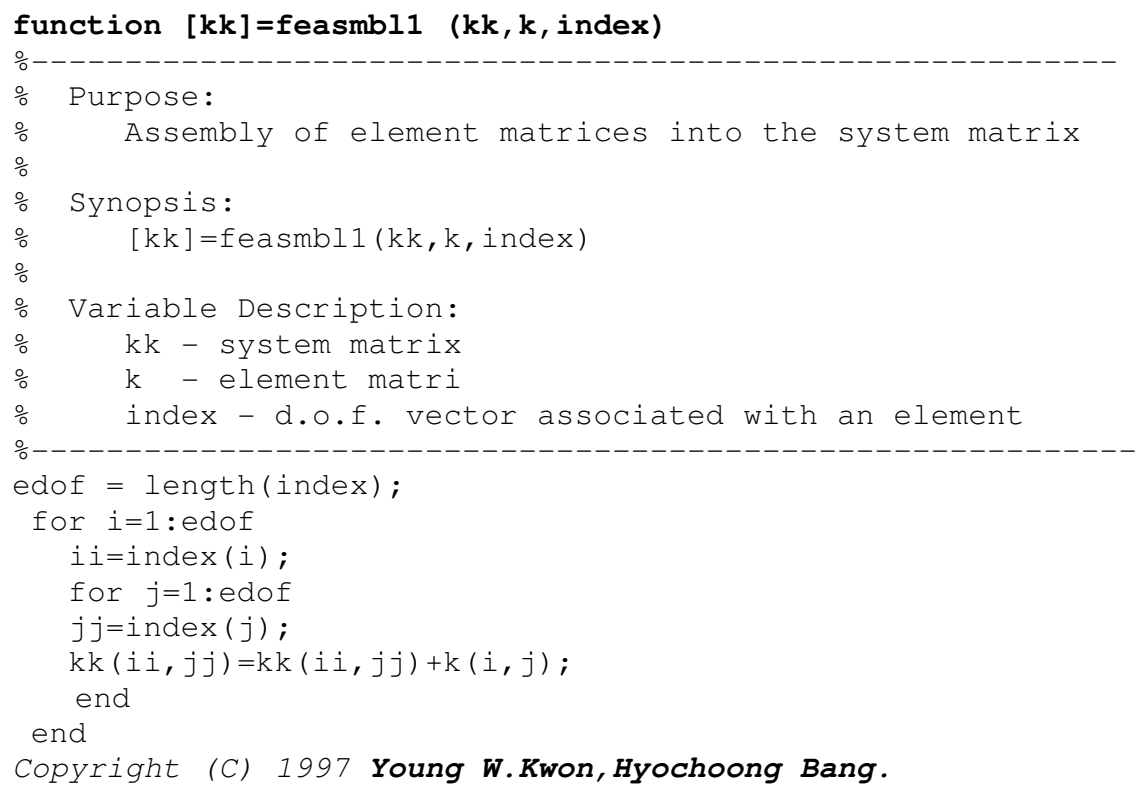

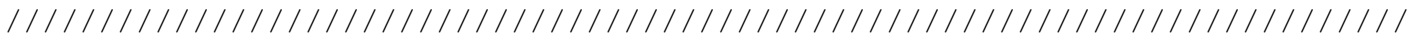

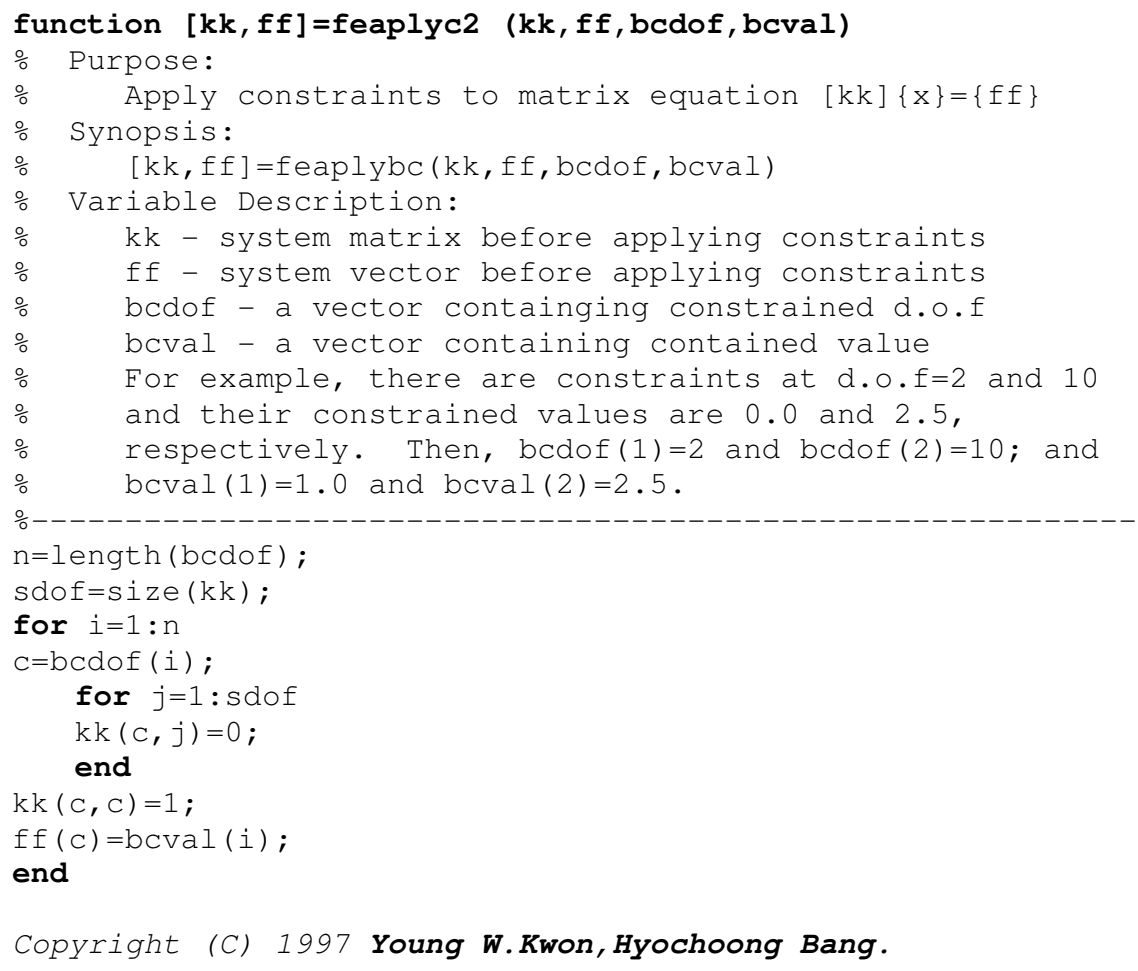


function $[D$, dwdp, dwdstrain, ecm $]=$ fajuste (gcoord, nodes, disp, a, b)

oobjetivo: Ajustar en la trayectoria estudiada, los corrimientos Raman con el oresultado numérico de deformaciones.

oVariables de entrada: gcoord y nodes, matrices de mallado; disp, vector de odesplazamientos nodales; a, radio horizontal de elipse; b, radio vertical de olipse.

oVariables de salida:D, diferencia cuadrada entre las medidas experimentales ode $d \square / d p$ y resultado numérico; dwdp, Valores numéricos de coeficientes de opresión (d $\left.\square / d p_{n u m}\right)$; dwdstrain, costantes de deformación potencial; ecm, error ocuadrático medio de ajustes.

oCarga los corrimientos Raman experimentales en la trayectoria estudiada응

load A1TOx.mat

obtención de deformaciones en la trayectoria estudiada

strainmef $1=$ coordenadaspathx (nodes, gcoord, a, b, disp, c) ;

oAjuste de corrimientos experimentales Raman con deformaciones numéricas.

[ecm, dwdstrain, dwdp] =ajustestrainx (strainmef1,ploteo, c) ;

$\mathrm{dwdp}=\mathrm{dwdp}{ }^{\prime}$

\% Cálculo de máxima diferencia cuadrática entre coeficiente d $\square / d p$ experimental oy numérico

$\mathrm{D}=\left(\max \left(\left[(\operatorname{dwdp}(1)-0.07)^{\wedge} 2,(\operatorname{dwdp}(2)-0.55)^{\wedge} 2,(\operatorname{dwdp}(3)-0.55)^{\wedge} 2,(\operatorname{dwdp}(4)-\right.\right.\right.$

$\left.\left.\left.0.11)^{\wedge} 2\right]\right)\right)^{\wedge} 0.5$;

end

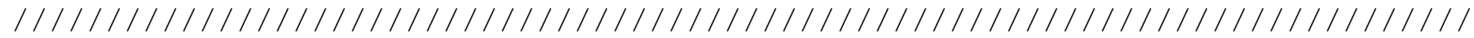

function strainmef=coordenadaspath (nodes, gcoord, a, b, disp, c)

oobjetivo: Extraer deformaciones de la trayectoria estudiada.

oVariables de entrada: gcoord y nodes, matrices de mallado; disp, vector de odesplazamientos nodales; a, radio horizontal de elipse; b, radio vertical de olipse; c, matriz con datos experimentales y coordenadas de trayectoria.

oVariables de salida: straimef, matriz que contiene deformaciones en la otrayectoria estudiada.

pathx $=\mathrm{c}(:, 1)$

epsilon=zeros $(3$, length $($ pathx $))$;

pathy=0 ;

deltay $=1 e-6 * a ; w=1$;

[nel, yor] =size (nodes);

for $w=1$ : length (pathx)

for $i e l=1:$ nel

$\mathrm{xi}=\operatorname{gcoord}($ nodes $(i e l, 1), 1)$;

$x d=\operatorname{gcoord}($ nodes $(i e l, 2), 1)$;

if gcoord(nodes(iel,2),2) < deltay

if $(\operatorname{pathx}(w)>=x i) \&(\operatorname{pathx}(w)<x d)$

$\mathrm{h}=\mathrm{xd}-\mathrm{xi}$;

$\mathrm{s}=-1$;

$l=p a t h x(w)-x i$;

$r=-(h-2 \star l) / h$;

disp $0=$ disp ;

else

epsilon $(:, w)=$ fun6 ( nodes, gcoord, dispo, iel, r, s) ;

end

else

end 


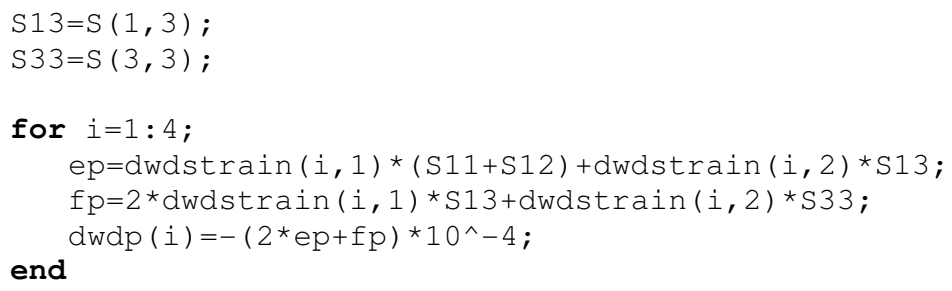


function $[p, t]=d i s t m e s h 2 d(f d, f h, h 0, b b o x, p f i x$, varargin)

DISTMESH2D 2-D Mesh Generator using Distance Functions. 응 $\quad[P, T]=D I S T M E S H 2 D(F D, F H, H O, B B O X, P F I X, F P A R A M S)$

T: Triangle indices (NTX3)

FD: Distance function $d(x, y)$

FH: Scaled edge length function $h(x, y)$

HO: Initial edge length

BBOX: Bounding box [xmin, ymin; xmax, ymax]

PFIX: Fixed node positions (NFIXX2)

FPARAMS: Additional parameters passed to FD and FH

o Copyright (C) 2004-2012 Per-olof Persson. See COPYRIGHT.TXT for odetails.

dptol $=.02$

ttol $=0.002$;

Fscale=1.15;

deltat $=.2$;

geps $=.001 * h 0$

deps=sqrt (eps) *h0; densityctrlfreq=30;

1. Create initial distribution in bounding box (equilateral otriangles)

$[x, y]=$ meshgrid $(\operatorname{bbox}(1,1): h 0: \operatorname{bbox}(2,1)$, bbox $(1,2): h 0 * \operatorname{sqrt}(3) / 2: \operatorname{bbox}(2,2))$;

$x(2: 2:$ end,$:)=x(2: 2:$ end, $:)+h 0 / 2$;

$p=[x(:), y(:)]$

o Shift even rows

․ 2. Remove points outside the region, apply the rejection method

$p=p($ feval (fd, $p, \operatorname{varargin}\{:\})<g e p s,:)$;

ro=1. /feval (fh, p, varargin $\{:\}) .{ }^{\wedge} 2 ; \quad \frac{\circ}{0}$ Probability to keep point

$p=p(\operatorname{rand}(\operatorname{size}(p, 1), 1)<r 0 . / \max (r 0),:) ; \quad \frac{\text { Rejection method }}{}$

if isempty (pfix), p=setdiff(p,pfix, 'rows');

end $\frac{\circ}{0}$ Remove duplicated nodes

pfix=unique (pfix, 'rows'); nfix=size(pfix,1);

$p=[p f i x ; p]$;

을 Prepend fix points

$N=\operatorname{size}(p, 1)$;

N Number of points $N$

count $=0$;

pold=inf;

Clf, view(2), axis equal, axis off

For first iteration

while 1

count $=$ count +1

ㅇ. Retriangulation by the Delaunay algorithm

if $\max (\operatorname{sqrt}(\operatorname{sum}((p-p o l d) \cdot \wedge 2,2)) / h 0)>$ ttol pold $=p$;

$t=$ delaunayn $(p)$;

․ Any large movement?

pmid $=(p(t), 1)$

List of triangles

$t=t(f e v a l(f d, p m i d$, varargin $\{:\})<-g e p s,:)$; $\frac{\circ}{0}$ Keep interior triangles

\% 4. Describe each bar by a unique pair of nodes

bars $=[t(:,[1,2]) ; t(:,[1,3]) ; t(:,[2,3])] ;$ o Interior bars duplicated

bars=unique (sort (bars,2), 'rows'); $\quad \frac{\circ}{0}$ Bars as node pairs

․ 5. Graphical output of the current mesh

cla,patch ('vertices', p, 'faces', t, 'edgecol', 'k', 'facecol', [.8,.9, 1]);

drawnow

end

6. Move mesh points based on bar lengths $L$ and forces $F$

barvec $=$ p (bars $(:, 1),:)-p(\operatorname{bars}(:, 2),:)$;

$L=\operatorname{sqrt}\left(\operatorname{sum}\left(\right.\right.$ barvec $\left.\left.{ }^{\wedge}{ }^{2} 2,2\right)\right)$;

ㄴ.st of bar vectors

hbars=feval $(f h,(p(\operatorname{bars}(:, 1),:)+p(\operatorname{bars}(:, 2),:)) / 2, \operatorname{varargin}\{:\})$

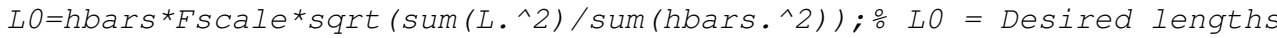

Density control - remove points that are too close

if mod(count, densityctrlfreq) $==0$ \& any $\left(L 0>2{ }^{\star} L\right)$ 


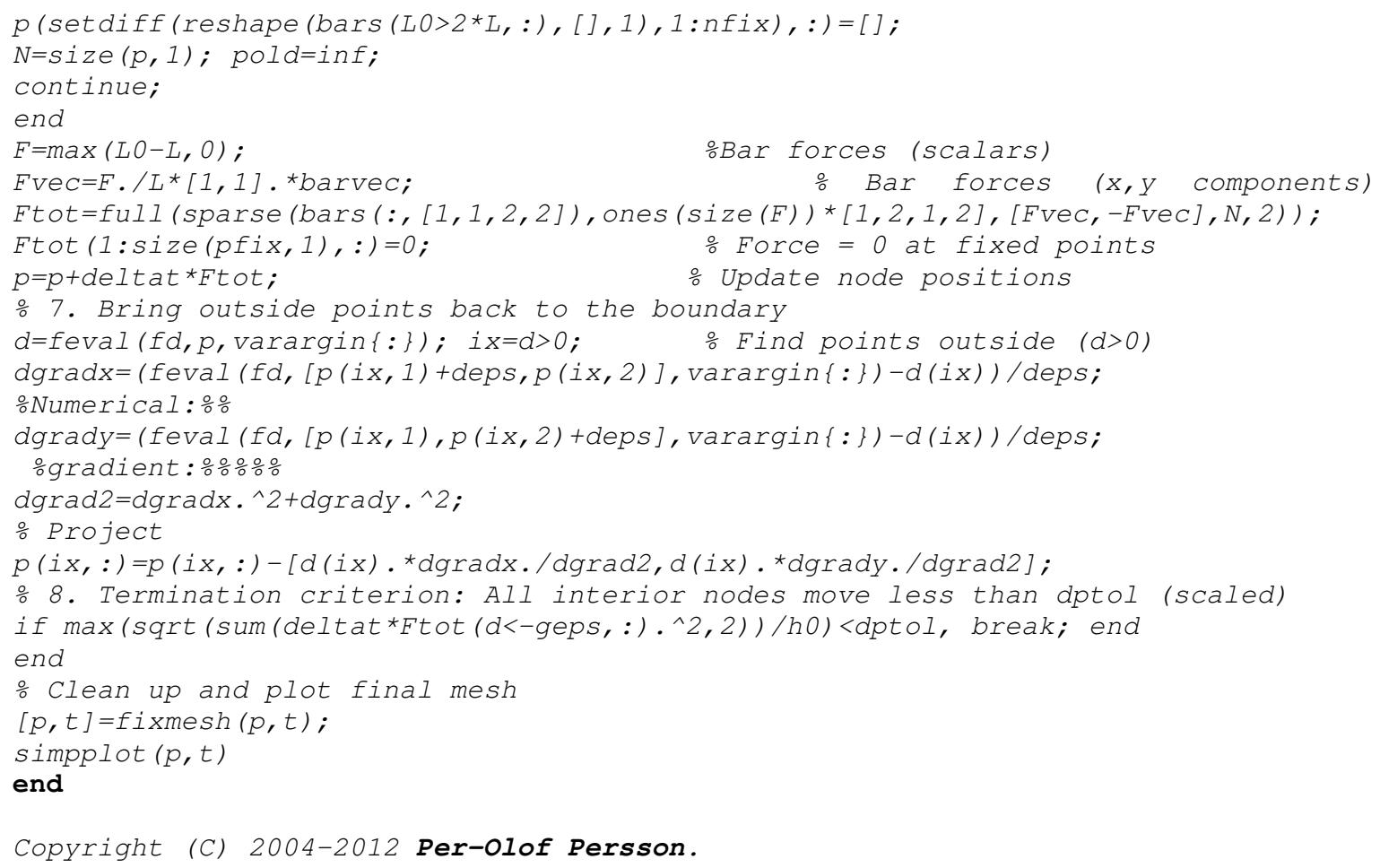

111111111111111111111111111111111111111111111111111111111111

function $[r, g$ coord, nodes, af, bf] $=\operatorname{selctr}(a, b)$

응 $\% \circ \% \%$

$\mathrm{ai}=[0.06,0.12,0.2: 0.1: 0.5,0.61,0.7: 0.1: 6]$;

bi=7*ones $(1$, length (ai));

ri=bi./ai;

$\mathrm{ro}=\mathrm{b} / \mathrm{a}$;

roi=ro*ones $(1$, length (ri));

delta=(abs (roi-ri));

deltamin=min (delta)

io=find (delta==deltamin);

if length(io) $>1$

i=io $(1)$;

else

i=io;

end

$\mathrm{k}=[$ 'gcnodes', num2str(i), '.mat']

load $(\mathrm{k})$;

$\mathrm{bf}=\mathrm{b}$;

$\mathrm{af}=\mathrm{b} / \mathrm{ri}$ (i);

ratio $=\mathrm{bf} / 7$;

$r=b f / a f$;

gcoord=ratio*gcoord;

end 
function [nodes, gcoord, disp]=codigo2 (parametros, gcoord, nodes)

\%०Objetivo: Función que calcula campo de deformaciones resultante de la \% $\%$ expansión de un cuarto de elipse, utiliza elementos triangulares de 3 nodos.

oVariables de entrada: vector parámetros de dimensiones 1x4; parámetros(1) y oparámetros(2), radio horizontal y vertical de elipse; parámetros(3) y oparámetros(4), expansiones horizontal y vertical; gcoord y nodes, matrices de omallado.

oVariables de salida: gcoord y nodes, matrices asociadas al mallado; disp, vector de desplazamientos nodales.

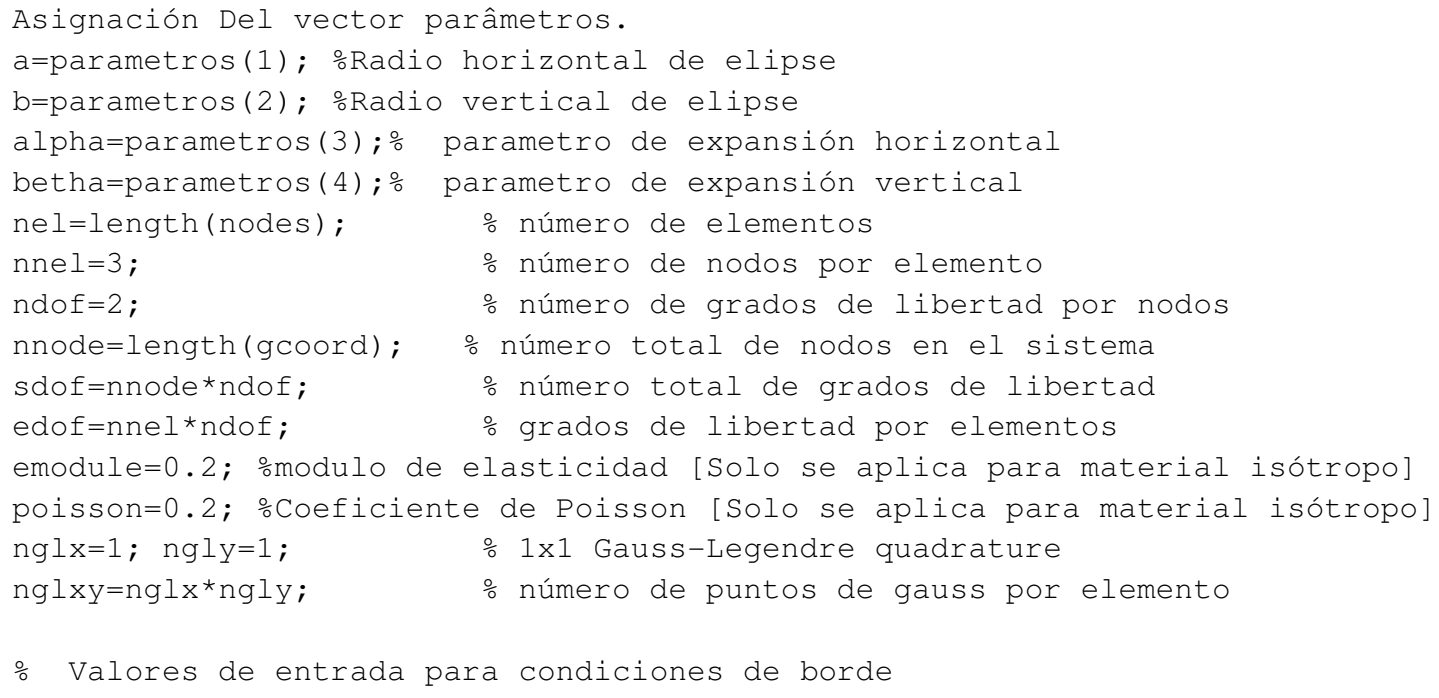




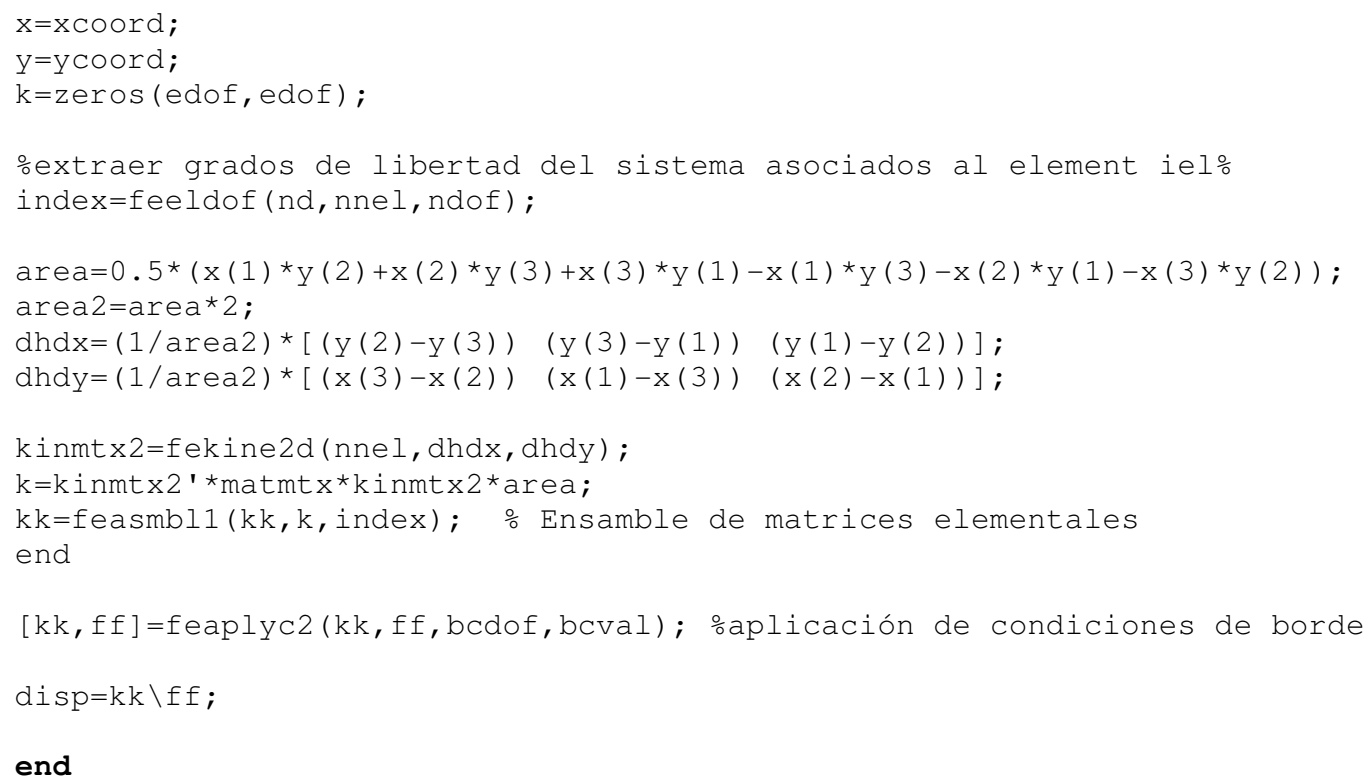




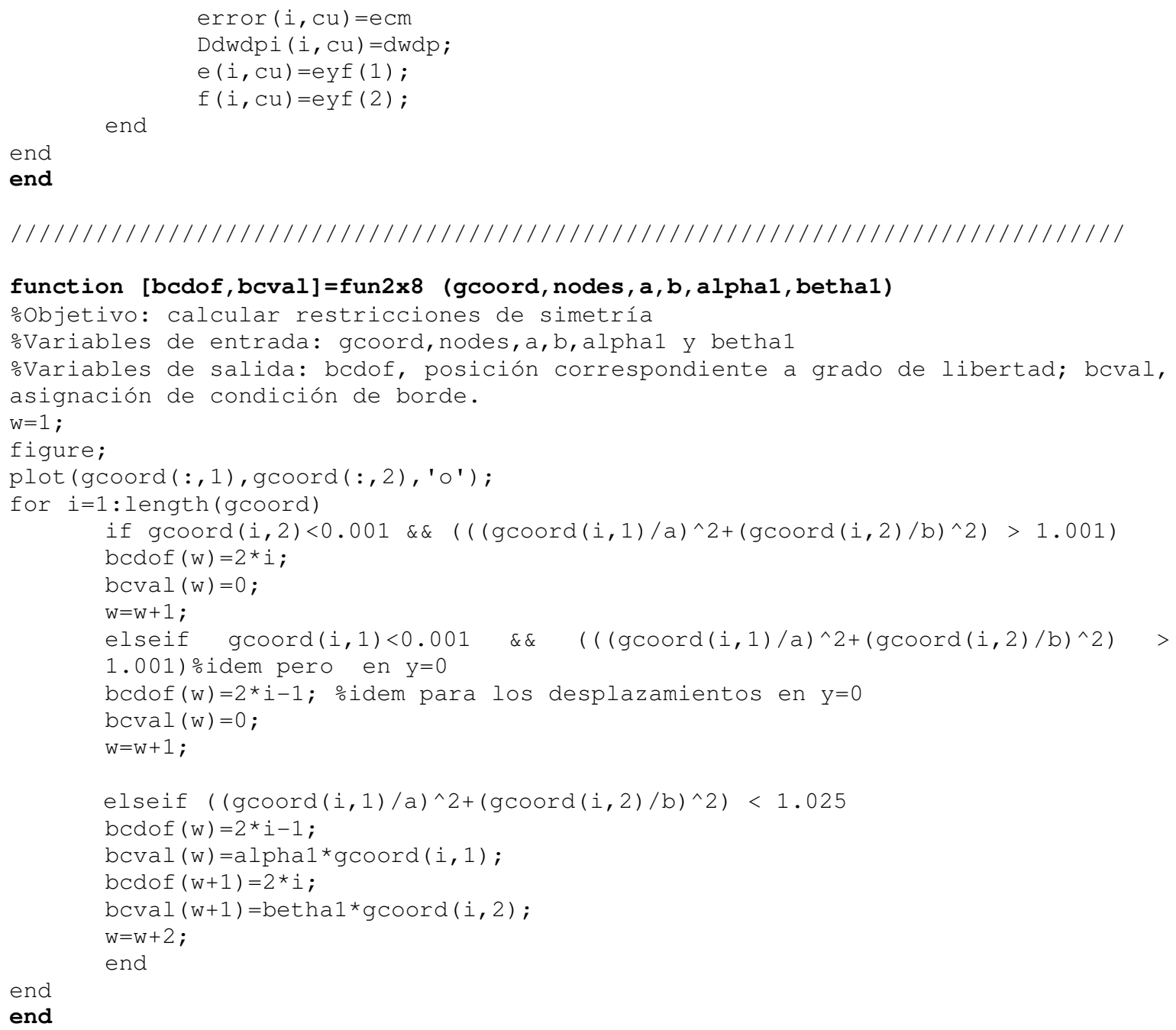




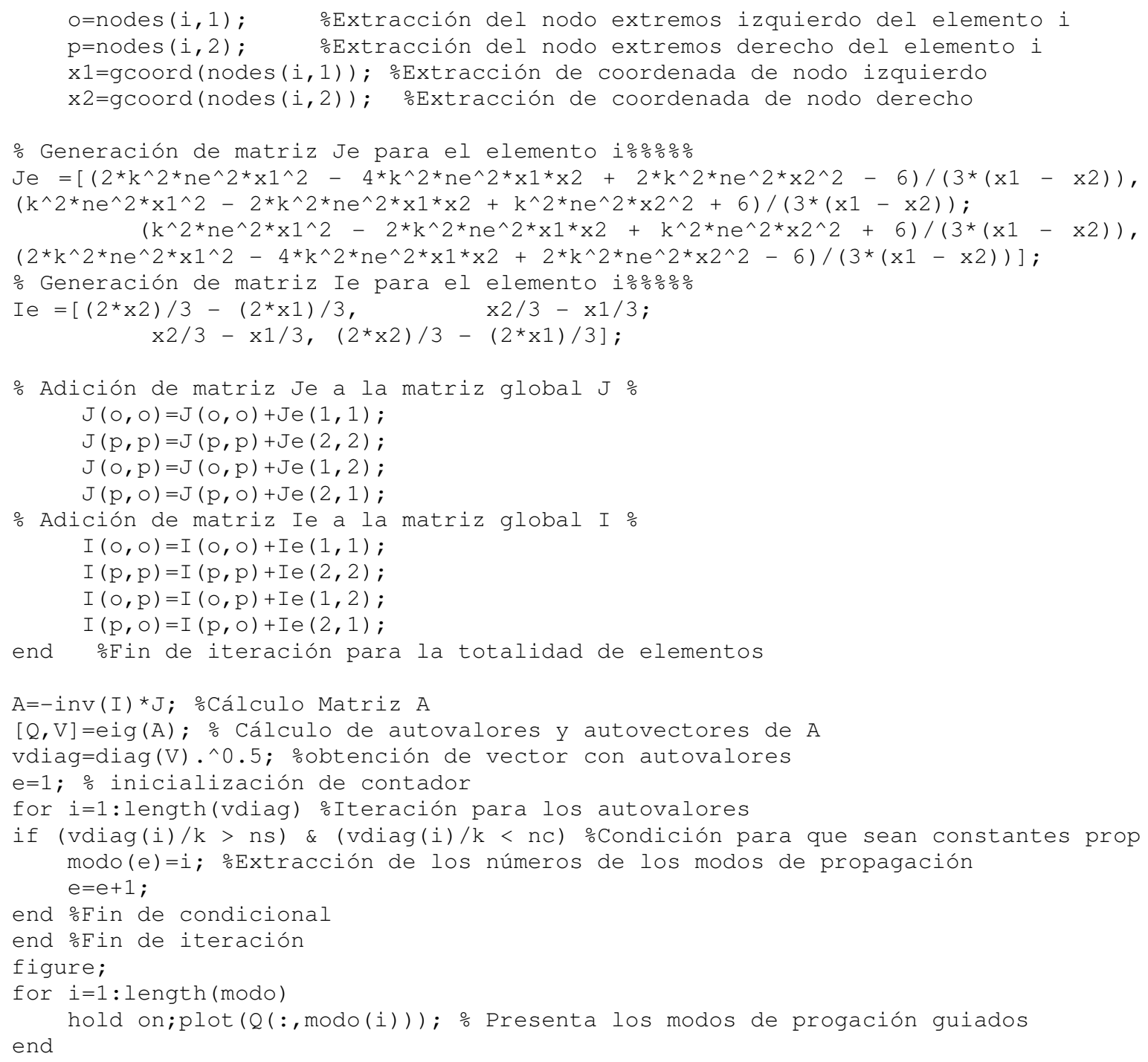


for $i=1$ :length (gcoord2)

$x i j=\operatorname{gcoord} 2(i, 1)$;

$y i j=\operatorname{gcoord} 2(i, 2)$;

if $\left(\left((x i j / a)^{\wedge} 2+(y i j / b)^{\wedge} 2\right)<1.01\right)$

ninodo $(i, 1)=2.30025-1 * 1 e-2$;

elseif $\left((y i j<0) \&\left(\left((x i j / a)^{\wedge} 2+(y i j / b)^{\wedge} 2\right)>1.01\right)\right)$

yij=-yij;

\% Determinación del elemento que contiene al nodo $i$.

iel=tsearch ( gcoord1 $(:, 1), \operatorname{gcoord}(:, 2), \operatorname{nodes} 1, x i j, y i j)$;

oCálculo de las coordenadas de cada esquina del elemento iel

$\mathrm{x} 1=\operatorname{gcoord} 1$ (nodes1 (iel, 1), 1);

$\mathrm{x} 2=\operatorname{gcoord} 1($ nodes $1($ iel, 2$), 1)$;

$\times 3=\operatorname{gcoord} 1($ nodes $1($ iel, 3$), 1)$;

y $1=$ gcoord1 (nodesl (iel, 1), 2);

y2= gcoord $1($ nodes $1($ iel, 2$), 2)$;

y3 $=$ gcoord 1 (nodes $1($ iel, 3$), 2)$;

dispel $=[\operatorname{disp}(2 *$ nodes $1(i e l, 1)-1) ; \operatorname{disp}(2 *$ nodes $1(i e l, 1)) ; \operatorname{disp}(2 *$ nodes $1(i e l, 2)-$

$1)$; disp $\left(2{ }^{\star}\right.$ nodesl $\left.(i e l, 2)\right)$; disp $\left(2{ }^{\star}\right.$ nodesl $\left.(i e l, 3)-1\right)$; disp $\left.(2 \star n o d e s 1(i e l, 3))\right]$;

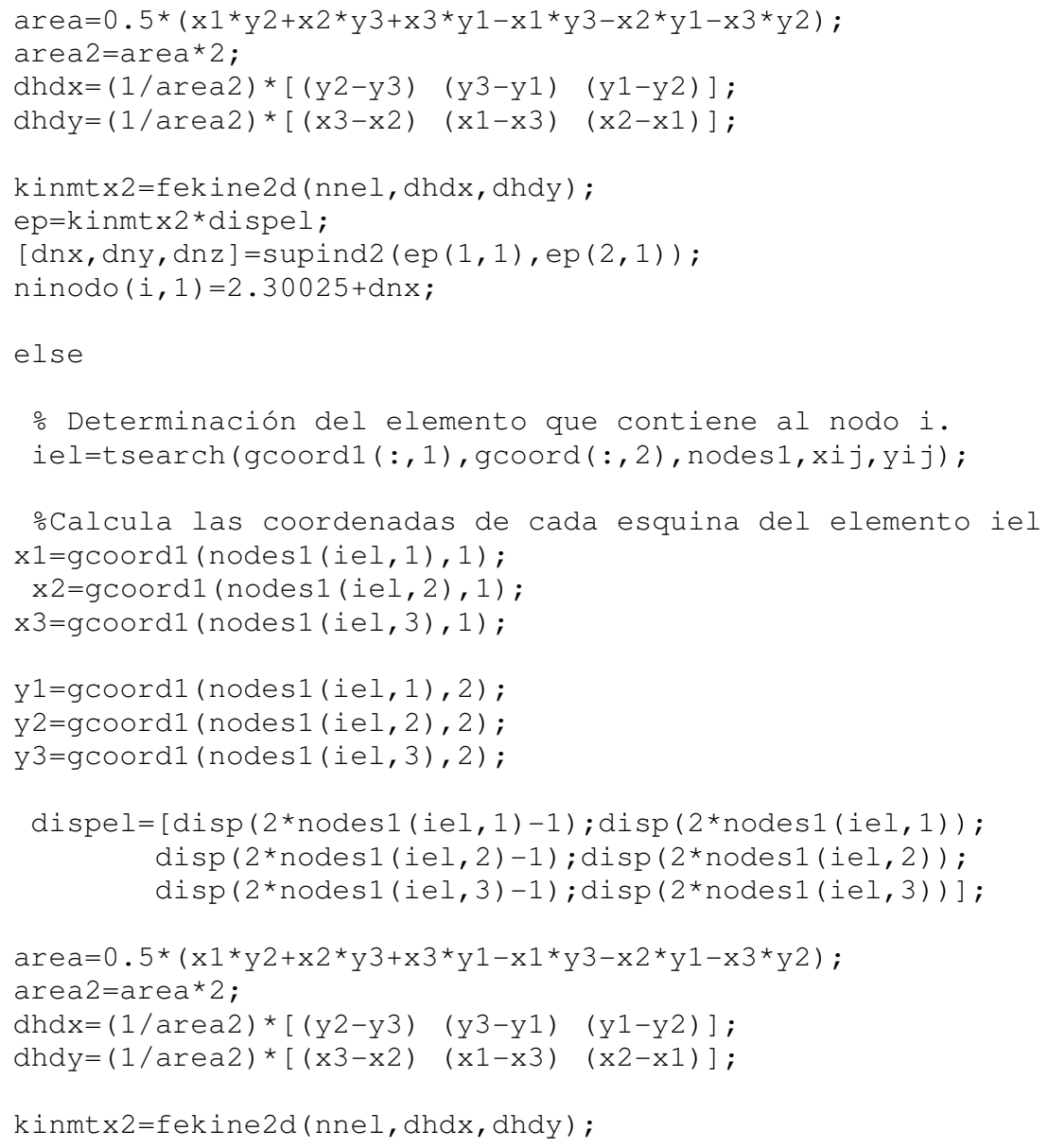




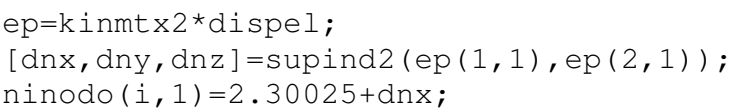

end

end

for $i=1:$ length (nodes 2$)$

niel $(i)=$ mean $([\operatorname{ninodo}(\operatorname{nodes} 2(i, 1)), \operatorname{ninodo}(\operatorname{nodes} 2(i, 2)), \operatorname{ninodo}(\operatorname{nodes} 2(i, 3))])$; end

$\mathrm{n}=\mathrm{niel}$;

$\operatorname{gcoord} 2=\operatorname{gcoord} 2 * 1 e-6$;

end

/////////////////////////////////////////////////////////////

function $[\mathrm{EE}]=$ codigo3 (gcoord2, nodes $2, \mathrm{n}, \mathrm{n} 0$, lambda)

objetivo: Calcular los modos guiados.

oVariables de entrada: gcoord, coordenadas de los nodos; nodes, oconectividad \%de los nodos; $n$, vector con valores de índice de orefracción promedio para \%cada elemento;

oVariable de salida: EE, Intesidad total de los modos de propagación ocorrespondiente a cada nodo;

$\mathrm{ns}=\mathrm{n} 0$

$\mathrm{nc}=\max (\mathrm{n})$;

$\mathrm{k}=2 * \mathrm{pi} / \mathrm{lambda}$;

J=zeros (length (gcoord), length (gcoord));

I=zeros (length (gcoord), length (gcoord));

for iel=1:length(nodes) Inicialización de iteración para cada elemento ne=n(iel); $\frac{\circ}{2}$ tracción de índice de refracción de element iel

Fil=nodes(iel,1); Oobtención del número global del nodo elemental 1 Fi2=nodes $(i e l, 2)$; $\%$ Idem para el nodo elemental 2

Fi3=nodes (iel,3); $\%$ Idem para el nodo elemental 3

$\mathrm{x} 1=\operatorname{gcoord}($ nodes $(\mathrm{iel}, 1), 1) ; \circ$ Coordenada $\mathrm{x}$ de nodo 1

$\times 2=$ gcoord (nodes $(i e l, 2), 1) ; \%$ Idem para nodo 2

$\times 3=\operatorname{gcoord}($ nodes $($ iel, 3$), 1) ; \%$ Idem para nodo 3

y $1=$ gcoord (nodes $(i e l, 1), 2) ;$ ocoordenada y de nodo 1

y2=gcoord (nodes $(i e l, 2), 2) ; \%$ Idem para nodo 2

y3 $=$ gcoord (nodes $($ iel, 3$), 2)$; $\%$ Idem para nodo 3

oCoordenada $x$ del centro geométrico del elemento iel.

$\mathrm{xg}=\left(\operatorname{gcoord}\left(\operatorname{nodes}\left(\mathrm{iel}_{1} 1\right), 1\right)+\operatorname{gcoord}(\operatorname{nodes}(\mathrm{iel}, 2), 1)+\operatorname{gcoord}(\operatorname{nodes}(\mathrm{iel}, 3), 1)\right) /$ 3 ;

oCoordenada $x$ del centro geométrico del elemento iel.

$\mathrm{yg}=\left(\operatorname{gcoord}\left(\operatorname{nodes}\left(\mathrm{iel}_{1} 1\right), 2\right)+\operatorname{gcoord}\left(\operatorname{nodes}\left(\mathrm{iel}_{1}, 2\right), 2\right)+\operatorname{gcoord}\left(\operatorname{nodes}\left(\mathrm{iel}_{1}, 3\right), 2\right)\right) /$ 3 ;

$\mathrm{Se}=0.5 *((\mathrm{x} 2-\mathrm{x} 1) *(\mathrm{y} 3-\mathrm{y} 1)-(\mathrm{x} 3-\mathrm{x} 1) *(\mathrm{y} 2-\mathrm{y} 1)) ;$ ․

- Constantes para el cálculo (Ver [Okamoto])

$\mathrm{Se} 1=\mathrm{Se}{ }^{*} \mathrm{xg} ; \mathrm{Se} 2=\mathrm{Se}{ }^{*} \mathrm{yg}$;

$\mathrm{Se} 3=3 / 4^{\star} \mathrm{xg} \mathrm{C}^{\star}{ }^{\star} \mathrm{Se}+\mathrm{Se}^{\star}\left(\mathrm{x} 1^{\wedge} 2+\mathrm{x} 2^{\wedge} 2+\mathrm{x} 3^{\wedge} 2\right) / 12 ;$

$\mathrm{Se} 4=3 / 4{ }^{*} \mathrm{yg}^{\wedge} 2^{\star} \mathrm{Se}^{\mathrm{S}} \mathrm{Se} e^{\star}\left(\mathrm{y} 1^{\wedge} 2+\mathrm{y} 2^{\wedge} 2+\mathrm{y} 3^{\wedge} 2\right) / 12 ;$

$\mathrm{Se} 5=3 / 4{ }^{*} \mathrm{yg}{ }^{*} \mathrm{xg}{ }^{*} \mathrm{Se}+\mathrm{Se}^{*}(\mathrm{y} 1 * \mathrm{x} 1+\mathrm{y} 2 * x 2+\mathrm{y} 3 * x 3) / 12$;

operaciones para determinar elemtentos de matriz elemental Je:

$\mathrm{Je}=\left[-1 / 12^{\star}\left(-6 * x 2^{\wedge} 2-6 * \times 3^{\wedge} 2-6 * \mathrm{y} 2^{\wedge} 2-\right.\right.$

$6^{\star} \mathrm{y} 3^{\wedge} 2+12^{\star} \mathrm{x} 2{ }^{\star} \mathrm{x} 3+\mathrm{k}^{\wedge} 2^{\star} \mathrm{n} \mathrm{e}^{\wedge} 2^{\star} \mathrm{x} 2^{\wedge} 2^{\star} \mathrm{y} 3^{\wedge} 2+\mathrm{k}^{\wedge} 2^{\star} \mathrm{n} \mathrm{e}^{\wedge} 2^{\star} \mathrm{y} 2^{\wedge} 2^{\star} \mathrm{x} 3^{\wedge} 2+\mathrm{k}^{\wedge} 2^{\star} \mathrm{ne} \mathrm{e}^{\wedge} 2^{\star} \mathrm{y} 3^{\wedge} 2^{\star} \mathrm{x} 1^{\wedge} 2+\mathrm{k}^{\wedge} 2^{\star} \mathrm{ne} \mathrm{e}^{\wedge} 2$

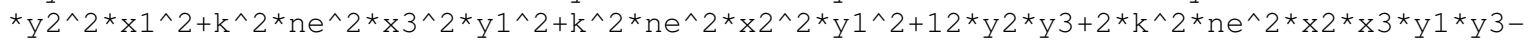




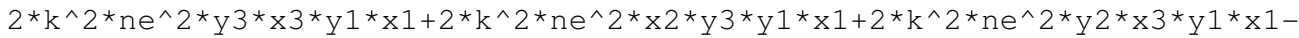

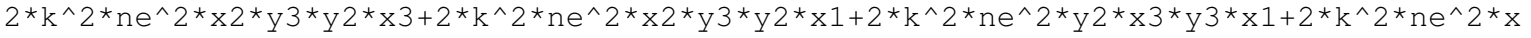

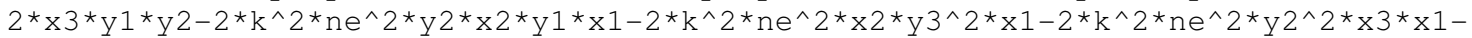

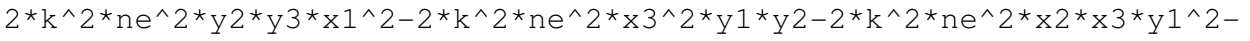

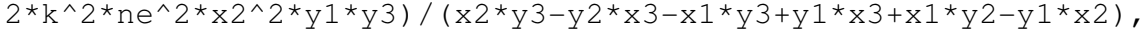

$1 / 24 *\left(12 * x 3^{\wedge} 2+12 * \mathrm{y} 3^{\wedge} 2+12 * x 1 * x 2-12 * x 1 * x 3-\right.$

$12^{\star} \mathrm{x} 2{ }^{\star} \mathrm{x} 3+\mathrm{k}^{\wedge} 2^{\star} \mathrm{n} \mathrm{e}^{\wedge} 2^{\star} \mathrm{x} 2^{\wedge} 2^{\star} \mathrm{y} 3^{\wedge} 2+\mathrm{k}^{\wedge} 2^{\star} \mathrm{n} \mathrm{e}^{\wedge} 2^{\star} \mathrm{y} 2^{\wedge} 2^{\star} \mathrm{x} 3^{\wedge} 2+\mathrm{k}^{\wedge} 2^{\star} \mathrm{ne} \mathrm{e}^{\wedge} 2^{\star} \mathrm{y} 3^{\wedge} 2^{\star} \mathrm{x} 1^{\wedge} 2+\mathrm{k}^{\wedge} 2^{\star} \mathrm{ne} e^{\wedge} 2^{\star} \mathrm{y} 2^{\wedge} 2^{\star} \mathrm{x}$

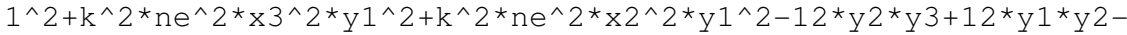

$12 * \mathrm{y} 1 * \mathrm{y} 3+2 * \mathrm{k} \wedge 2 * \mathrm{ne} e^{\wedge} 2 * x 2 * x 3 * \mathrm{y} 1 * \mathrm{y} 3-$

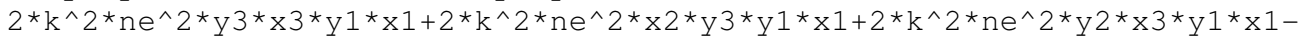

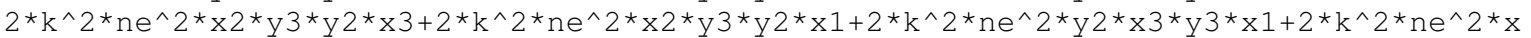

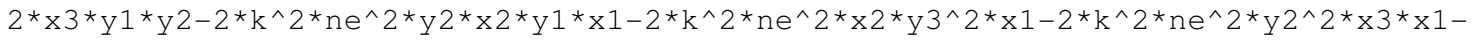

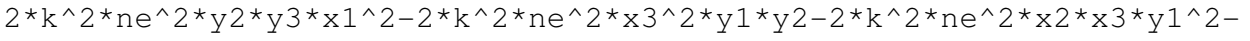

$\left.2 * k^{\wedge} 2 * n e^{\wedge} 2 * x 2 \wedge 2 * y 1 * y 3\right) /(x 2 * y 3-y 2 * x 3-x 1 * y 3+y 1 * x 3+x 1 * y 2-y 1 * x 2)$

$1 / 24 *\left(12 * x 2^{\wedge} 2+12^{\star} \mathrm{y} 2^{\wedge} 2-12 * x 1 * x 2+12^{\star} \times 1 * x 3-\right.$

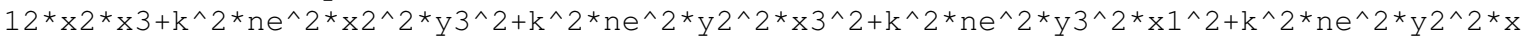

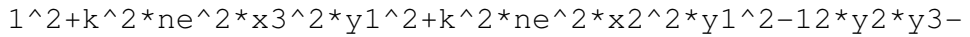

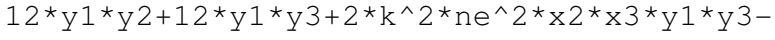

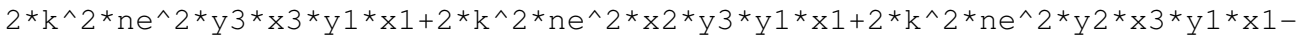

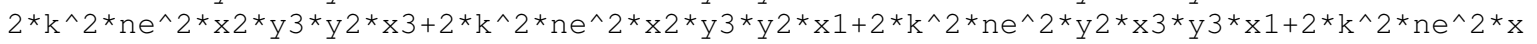

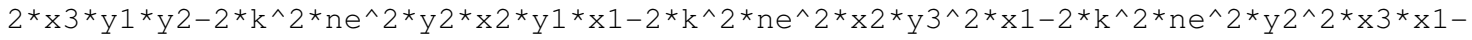

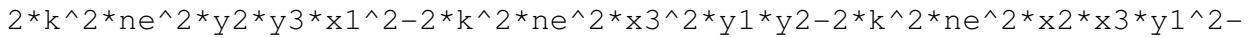

$\left.2 * \mathrm{k}^{\wedge} 2 \star \mathrm{n} \mathrm{e}^{\wedge} 2 * \mathrm{x} 22^{\wedge}{ }^{\star} \mathrm{y} 1 * \mathrm{y} 3\right) /(\mathrm{x} 2 * \mathrm{y} 3-\mathrm{y} 2 * \mathrm{x} 3-\mathrm{x} 1 * \mathrm{y} 3+\mathrm{y} 1 * \mathrm{x} 3+\mathrm{x} 1 * \mathrm{y} 2-\mathrm{y} 1 * \mathrm{x} 2) ;-$

$1 / 24 *\left(12 * x 3^{\wedge} 2+12^{*} \mathrm{y}^{\wedge} 2+12^{*} \mathrm{x} 1^{*} \mathrm{x} 2-12^{*} \mathrm{x} 1^{*} \times 3-\right.$

$12^{\star} \mathrm{x} 2{ }^{\star} \mathrm{x} 3+\mathrm{k}^{\wedge} 2^{\star} \mathrm{n} \mathrm{e}^{\wedge} 2^{\star} \mathrm{x} 2^{\wedge} 2^{\star} \mathrm{y} 3^{\wedge} 2+\mathrm{k}^{\wedge} 2^{\star} \mathrm{n} \mathrm{e}^{\wedge} 2^{\star} \mathrm{y} 2^{\wedge} 2^{\star} \mathrm{x} 3^{\wedge} 2+\mathrm{k}^{\wedge} 2^{\star} \mathrm{n} \mathrm{e}^{\wedge} 2^{\star} \mathrm{y} 3^{\wedge} 2^{\star} \mathrm{x} 1^{\wedge} 2+\mathrm{k}^{\wedge} 2^{\star} \mathrm{ne} e^{\wedge} 2^{\star} \mathrm{y} 2^{\wedge} 2^{\star} \mathrm{x}$ $1^{\wedge} 2+\mathrm{k}^{\wedge} 2^{\star} \mathrm{n} \mathrm{e}^{\wedge} 2^{\star} \mathrm{x} 3^{\wedge} 2^{\star} \mathrm{y} 1^{\wedge} 2+\mathrm{k}^{\wedge} 2^{\star} \mathrm{n} \mathrm{e}^{\wedge} 2^{\star} \mathrm{x} 2^{\wedge} 2^{\star} \mathrm{y} 1^{\wedge} 2-12^{\star} \mathrm{y} 2^{\star} \mathrm{y} 3+12^{\star} \mathrm{y} 1^{\star} \mathrm{y} 2-$

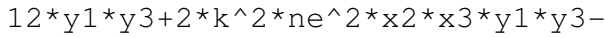

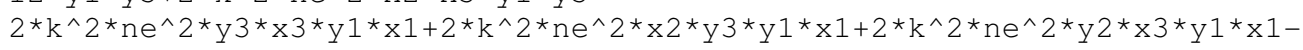

$2^{\star} \mathrm{k}^{\wedge} 2^{\star} \mathrm{n} \mathrm{e}^{\wedge} 2^{\star} \mathrm{x} 2^{\star} \mathrm{y} 3^{\star} \mathrm{y} 2^{\star} \mathrm{x} 3+2^{\star} \mathrm{k}^{\wedge} 2^{\star} \mathrm{n} \mathrm{e}^{\wedge} 2^{\star} \mathrm{x} 2^{\star} \mathrm{y}^{3}{ }^{\star} \mathrm{y} 2^{\star} \mathrm{x} 1+2^{\star} \mathrm{k}^{\wedge} 2^{\star} \mathrm{n} \mathrm{e}^{\wedge} 2^{\star} \mathrm{y} 2^{\star} \mathrm{x} 3{ }^{\star} \mathrm{y} 3^{\star} \mathrm{x} 1+2^{\star} \mathrm{k}^{\wedge} 2^{\star} \mathrm{n} \mathrm{e}^{\wedge} 2^{\star} \mathrm{x}$

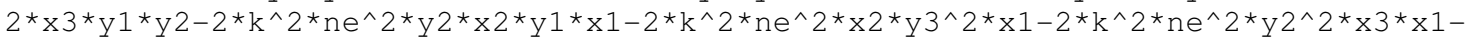

$2 * k^{\wedge} 2{ }^{*} n e^{\wedge} 2 * y 2 * y 3 * x 1 \wedge 2-2 * k^{\wedge} 2 * n e^{\wedge} 2 * x 3 \wedge 2 * y 1 * y 2-2 * k \wedge 2 * n e^{\wedge} 2 * x 2 * x 3 * y 1 \wedge 2-$

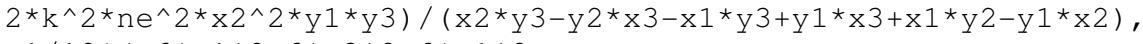

$-1 / 12^{*}\left(-6 * x 1^{\wedge} 2-6 * x 3^{\wedge} 2-6 * y 1^{\wedge} 2-\right.$

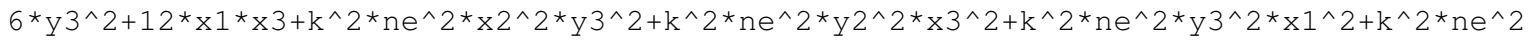
${ }^{\star} \mathrm{y} 2^{\wedge} 2^{\star} \mathrm{x} 1^{\wedge} 2+\mathrm{k}^{\wedge} 2^{\star} \mathrm{n} \mathrm{e}^{\wedge} 2^{\star} \mathrm{x} 3^{\wedge} 2^{\star} \mathrm{y} 1^{\wedge} 2+\mathrm{k}^{\wedge} 2^{\star} \mathrm{n} \mathrm{e}^{\wedge} 2^{\star} \mathrm{x} 2^{\wedge} 2^{\star} \mathrm{y} 1^{\wedge} 2+12{ }^{\star} \mathrm{y} 1^{\star} \mathrm{y} 3+2^{\star} \mathrm{k}^{\wedge} 2^{\star} \mathrm{n} e^{\wedge} 22^{\star} \mathrm{x} 2{ }^{\star} \mathrm{x} 33^{\star} \mathrm{y} 1^{\star} \mathrm{y} 3-$

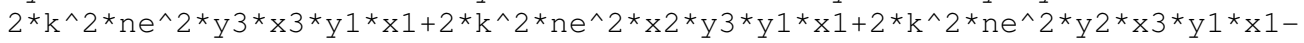

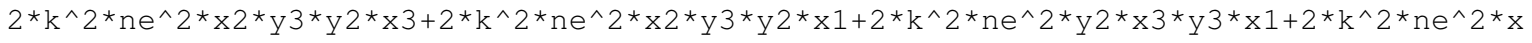

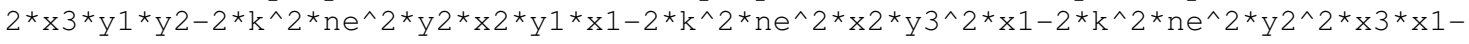

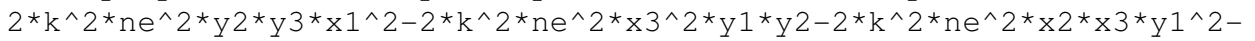

$\left.2^{\star} \mathrm{k}^{\wedge} 2^{\star} \mathrm{n} \mathrm{e}^{\wedge} 2^{\star} \mathrm{x} 2^{\wedge} 2^{\star} \mathrm{y} 1^{\star} \mathrm{y} 3\right) /\left(\mathrm{x} 2{ }^{\star} \mathrm{y} 3-\mathrm{y} 2^{\star} \times 3-\mathrm{x} 1{ }^{\star} \mathrm{y} 3+\mathrm{y} 1{ }^{\star} \mathrm{x} 3+\mathrm{x} 1^{\star} \mathrm{y} 2-\mathrm{y} 1^{\star} \mathrm{x} 2\right),-$

$1 / 24 *\left(12 * x 1^{\wedge} 2+12^{*} \mathrm{y} 1^{\wedge} 2-12^{*} \times 1 * x 2-\right.$

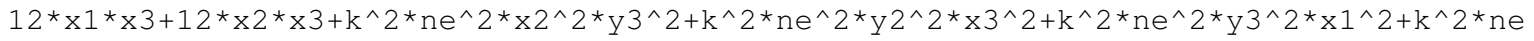
${ }^{\wedge} 2^{\star} \mathrm{y} 2^{\wedge} 2^{\star} \mathrm{x} 11^{\wedge} 2+\mathrm{k}^{\wedge} 2^{\star} \mathrm{n} \mathrm{e}^{\wedge} 2^{\star} \mathrm{x} 3^{\wedge} 2^{\star} \mathrm{y} 1^{\wedge} 2+\mathrm{k}^{\wedge} 2^{\star} \mathrm{n} \mathrm{e}^{\wedge} 2^{\star} \mathrm{x} 22^{\wedge} 2^{\star} \mathrm{y} 1^{\wedge} 2+12^{\star} \mathrm{y} 2{ }^{\star} \mathrm{y} 3-12^{\star} \mathrm{y} 1^{\star} \mathrm{y} 2-$

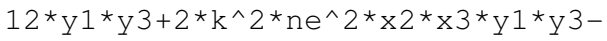

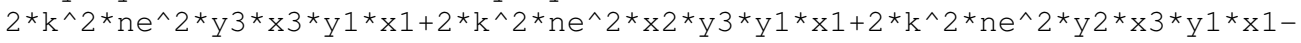

$2^{\star} \mathrm{k}^{\wedge} 2^{\star} \mathrm{n} \mathrm{e}^{\wedge} 2^{\star} \mathrm{x} 2{ }^{\star} \mathrm{y} 3^{\star} \mathrm{y} 2^{\star} \mathrm{x} 3+2^{\star} \mathrm{k}^{\wedge} 2^{\star} \mathrm{n} \mathrm{e}^{\wedge} 2^{\star} \mathrm{x} 2{ }^{\star} \mathrm{y} 3^{\star} \mathrm{y} 2^{\star} \mathrm{x} 1+2{ }^{\star} \mathrm{k}^{\wedge} 2^{\star} \mathrm{n} \mathrm{e}^{\wedge} 2^{\star} \mathrm{y} 2^{\star} \mathrm{x} 3{ }^{\star} \mathrm{y} 3{ }^{\star} \mathrm{x} 1+2^{\star} \mathrm{k}^{\wedge} 2^{\star} \mathrm{n} e^{\wedge} 2^{\star} \mathrm{x}$

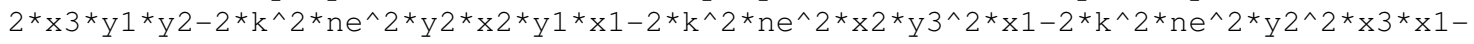

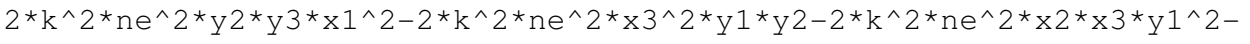

$\left.2 * k^{\wedge} 2 * n e^{\wedge} 2 * x 2 \wedge 2 * y 1 * y 3\right) /(x 2 * y 3-y 2 * x 3-x 1 * y 3+y 1 * x 3+x 1 * y 2-y 1 * x 2) ;-$

$1 / 24 *\left(12 * x 2 \wedge 2+12 * \mathrm{y} 22^{\wedge} 2-12 * x 1 * x 2+12 * x 1 * x 3-\right.$

$12^{\star} \mathrm{x} 22^{\star} \mathrm{x} 3+\mathrm{k}^{\wedge} 2^{\star} \mathrm{n} \mathrm{e}^{\wedge} 2^{\star} \mathrm{x} 2^{\wedge} 2^{\star} \mathrm{y} 3^{\wedge} 2+\mathrm{k}^{\wedge} 2^{\star} \mathrm{n} e^{\wedge} 2^{\star} \mathrm{y} 2^{\wedge} 2{ }^{\star} \mathrm{x} 3^{\wedge} 2+\mathrm{k}^{\wedge} 2^{\star} \mathrm{n} \mathrm{e}^{\wedge} 2^{\star} \mathrm{y} 3^{\wedge} 2^{\star} \mathrm{x} 1^{\wedge} 2+\mathrm{k}^{\wedge} 2^{\star} \mathrm{n} e^{\wedge} 2^{\star} \mathrm{y} 2^{\wedge} 2^{\star} \mathrm{x}$

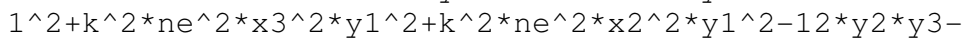

$12 * y 1 * y 2+12 * y 1 * y 3+2 * k^{\wedge} 2 * n e^{\wedge} 2 * x 2 * x 3 * y 1 * y 3-$

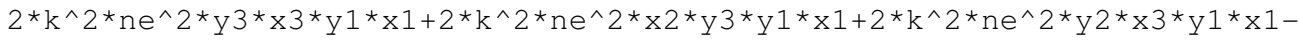

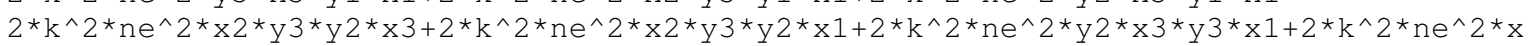

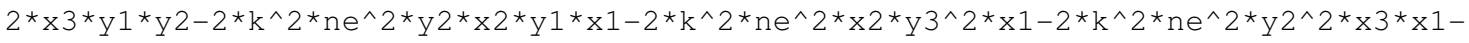

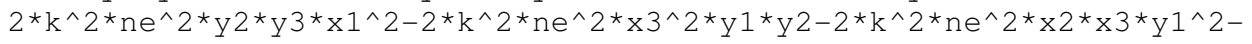

$\left.2 * k^{\wedge} 2 * n e^{\wedge} 2 * x 2 \wedge 2 * y 1 * y 3\right) /(x 2 * y 3-y 2 * x 3-x 1 * y 3+y 1 * x 3+x 1 * y 2-y 1 * x 2),-$

$1 / 24 *\left(12 * x 1^{\wedge} 2+12 * y 1^{\wedge} 2-12 * x 1 * x 2-\right.$ 


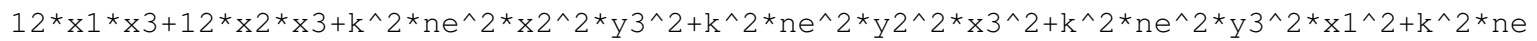

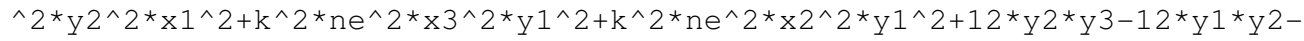

$12 * \mathrm{y} 1 * \mathrm{y} 3+2 * \mathrm{k} 2^{\star} 2 \mathrm{ne}^{\wedge} 2 * \mathrm{x} 2 * \mathrm{x} 3{ }^{*} \mathrm{y} 1{ }^{*} \mathrm{y} 3-$

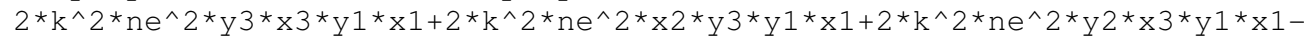

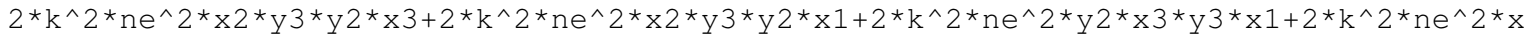

$2^{\star} \mathrm{x} 3^{\star} \mathrm{y} 1^{\star} \mathrm{y} 2-2^{\star} \mathrm{k}^{\wedge} 2^{\star} \mathrm{n} \mathrm{e}^{\wedge} 2^{\star} \mathrm{y} 2{ }^{\star} \mathrm{x} 22^{\star} \mathrm{y} 1^{\star} \mathrm{x} 1-2^{\star} \mathrm{k}^{\wedge} 2^{\star} \mathrm{n} \mathrm{e}^{\wedge} 2^{\star} \mathrm{x} 22^{\star} \mathrm{y} 3^{\wedge} 2^{\star} \mathrm{x} 1-2^{\star} \mathrm{k}^{\wedge} 2^{\star} \mathrm{n} \mathrm{e}^{\wedge} 2^{\star} \mathrm{y} 2^{\wedge} 2^{\star} \mathrm{x} 3^{\star} \mathrm{x} 1-$

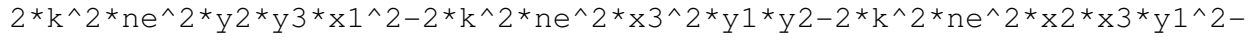

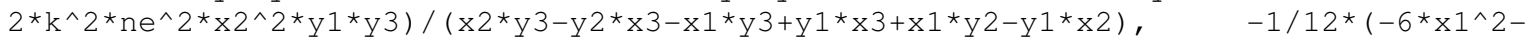
$6 * x 2^{\wedge} 2-6 * y 1 \wedge 2-$

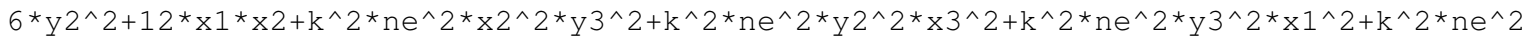

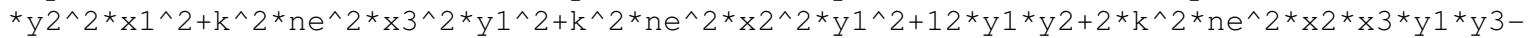
$2{ }^{*} \mathrm{k}^{\wedge} 2 \mathrm{ne}^{\wedge} 2{ }^{*} \mathrm{y} 3{ }^{*} \mathrm{x} 3{ }^{*} \mathrm{y} 1{ }^{*} \mathrm{x} 1+2{ }^{*} \mathrm{k} 2^{*} \mathrm{n} \mathrm{e}^{\wedge} 2{ }^{*} \mathrm{x} 2{ }^{*} \mathrm{y} 3{ }^{*} \mathrm{y} 1{ }^{*} \mathrm{x} 1+2{ }^{*} \mathrm{k} 2^{*} \mathrm{n} e^{\wedge} 2{ }^{*} \mathrm{y} 2{ }^{*} \mathrm{x} 3{ }^{*} \mathrm{y} 1{ }^{*} \mathrm{x} 1-$

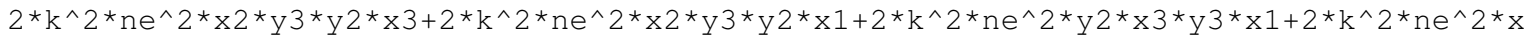

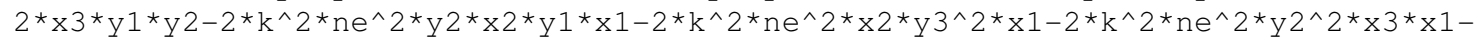

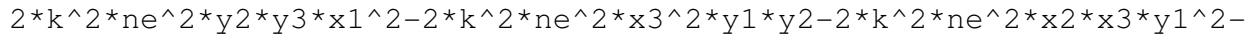

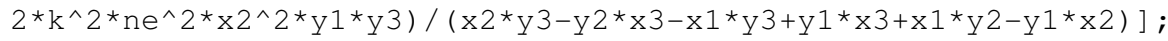

ooperaciones para obtener elementos de matriz Ie:

$\mathrm{Ie}=[1 / 12 * x 2 * y 3-1 / 12 * y 2 * x 3-1 / 12 * x 1 * y 3+1 / 12 * y 1 * x 3+1 / 12 * x 1 * y 2-1 / 12 * y 1 * x 2$,

$1 / 24 * x 2 * y 3-1 / 24 * y 2 * x 3-1 / 24 * x 1 * y 3+1 / 24 * y 1 * x 3+1 / 24 * x 1 * y 2-1 / 24 * y 1 * x 2, \quad 1 / 24 * x 2 * y 3-$

$1 / 24 * \mathrm{y} 2 * \mathrm{x} 3-1 / 24{ }^{*} \mathrm{x} 1 * \mathrm{y} 3+1 / 24 * \mathrm{y} 1 * \mathrm{x} 3+1 / 24 * \mathrm{x} 1 * \mathrm{y} 2-1 / 24{ }^{*} \mathrm{y} 1{ }^{*} \mathrm{x} 2 ; 1 / 24 * \mathrm{x} 2 * \mathrm{y} 3-1 / 24{ }^{*} \mathrm{y} 2{ }^{*} \mathrm{x} 3-$

$1 / 24 * x 1 * y 3+1 / 24 * y 1 * x 3+1 / 24 * x 1 * y 2-1 / 24 * y 1 * x 2,1 / 12 * x 2 * y 3-1 / 12 * y 2 * x 3-$

$1 / 12 * x 1 * y 3+1 / 12 * y 1 * x 3+1 / 12 * x 1 * y 2-1 / 12 * y 1 * x 2,1 / 24 * x 2 * y 3-1 / 24 * y 2 * x 3-$

$1 / 24 * x 1 * y 3+1 / 24 * y 1 * x 3+1 / 24 * x 1 * y 2-1 / 24 * y 1 * x 2 ; \quad 1 / 24 * x 2 * y 3-1 / 24 * y 2 * x 3-$

$1 / 24 * x 1 * y 3+1 / 24 * y 1 * x 3+1 / 24 * x 1 * y 2-1 / 24 * y 1 * x 2,1 / 24 * x 2 * y 3-1 / 24 * y 2 * x 3-$

$1 / 24 * x 1 * y 3+1 / 24 * y 1 * x 3+1 / 24 * x 1 * y 2-1 / 24 * y 1 * x 2,1 / 12 * x 2 * y 3-1 / 12 * y 2 * x 3-$

$1 / 12 * x 1 * y 3+1 / 12 * y 1 * x 3+1 / 12 * x 1 * y 2-1 / 12 * y 1 * x 2]$

oEnsamble de matrices elementales Je y Ie para obtener las matrices globales J \%y I.

$\mathrm{J}(\mathrm{Fi1}, \mathrm{Fil})=\mathrm{J}(\mathrm{Fi1}, \mathrm{Fil})+\mathrm{Je}(1,1)$;

$\mathrm{J}(\mathrm{Fi1}, \mathrm{Fi2})=\mathrm{J}(\mathrm{Fi1}, \mathrm{Fi2})+\mathrm{Je}(1,2)$;

$\mathrm{J}(\mathrm{Fi1}, \mathrm{Fi3})=\mathrm{J}(\mathrm{Fi1}, \mathrm{Fi3})+\mathrm{Je}(1,3)$;

$\mathrm{J}(\mathrm{Fi2}, \mathrm{Fi1})=\mathrm{J}(\mathrm{Fi2}, \mathrm{Fi1})+\mathrm{Je}(2,1)$;

$\mathrm{J}(\mathrm{Fi2}, \mathrm{Fi2})=\mathrm{J}(\mathrm{Fi2}, \mathrm{Fi2})+\mathrm{Je}(2,2)$;

$\mathrm{J}(\mathrm{Fi2}, \mathrm{Fi3})=\mathrm{J}(\mathrm{Fi2}, \mathrm{Fi3})+\mathrm{Je}(2,3)$;

$\mathrm{J}(\mathrm{Fi3}, \mathrm{Fi1})=\mathrm{J}(\mathrm{Fi3}, \mathrm{Fi1})+\mathrm{Je}(3,1)$;

$\mathrm{J}(\mathrm{Fi3}, \mathrm{Fi2})=\mathrm{J}(\mathrm{Fi3}, \mathrm{Fi2})+\mathrm{Je}(3,2)$;

$\mathrm{J}(\mathrm{Fi3}, \mathrm{Fi3})=\mathrm{J}(\mathrm{Fi3}, \mathrm{Fi3})+\mathrm{Je}(3,3)$;

$\mathrm{I}(\mathrm{Fi1}, \mathrm{Fi1})=\mathrm{I}(\mathrm{Fi1}, \mathrm{Fi1})+\mathrm{Ie}(1,1)$;

$\mathrm{I}(\mathrm{Fi1}, \mathrm{Fi2})=\mathrm{I}(\mathrm{Fi1}, \mathrm{Fi2})+\operatorname{Ie}(1,2)$;

$\mathrm{I}(\mathrm{Fi1}, \mathrm{Fi3})=\mathrm{I}(\mathrm{Fi1}, \mathrm{Fi3})+\operatorname{Ie}(1,3)$;

$\mathrm{I}(\mathrm{Fi2}, \mathrm{Fi1})=\mathrm{I}(\mathrm{Fi2}, \mathrm{Fi1})+\mathrm{Ie}(2,1)$;

$\mathrm{I}(\mathrm{Fi2}, \mathrm{Fi2})=\mathrm{I}(\mathrm{Fi2}, \mathrm{Fi2})+\mathrm{Ie}(2,2)$;

$\mathrm{I}(\mathrm{Fi2}, \mathrm{Fi3})=\mathrm{I}(\mathrm{Fi2}, \mathrm{Fi3})+\operatorname{Ie}(2,3)$;

$\mathrm{I}(\mathrm{Fi3}, \mathrm{Fi1})=\mathrm{I}(\mathrm{Fi3}, \mathrm{Fi1})+\operatorname{Ie}(3,1)$;

$\mathrm{I}(\mathrm{Fi3}, \mathrm{Fi2})=\mathrm{I}(\mathrm{Fi3}, \mathrm{Fi2})+\operatorname{Ie}(3,2)$;

$\mathrm{I}(\mathrm{Fi3}, \mathrm{Fi3})=\mathrm{I}(\mathrm{Fi3}, \mathrm{Fi3})+\operatorname{Ie}(3,3)$;

end ․ㅡㄹ de iteración sobre cada elemento

$A=-i n v(I) * J$; $\frac{\circ}{0}$ Cálculo de matriz $A$

$[\mathrm{V}, \mathrm{D}]=$ eig $(\mathrm{A}) ;$ o Cálculo de autovalores y autovectores de matriz A

\% Obtención del vector vdiag que contiene los oautovalores de A

vdiag=diag $(D) \cdot \wedge 0.5$;

$\mathrm{e}=1$;

for $i=1$ :length(vdiag) oIteración para todos los autovalores

- Condición para definir si es oun modo guiado

if $(\operatorname{vdiag}(\mathrm{i}) / \mathrm{k}>\mathrm{ns}) \&(\operatorname{vdiag}(\mathrm{i}) / \mathrm{k}<\mathrm{nc})$ 


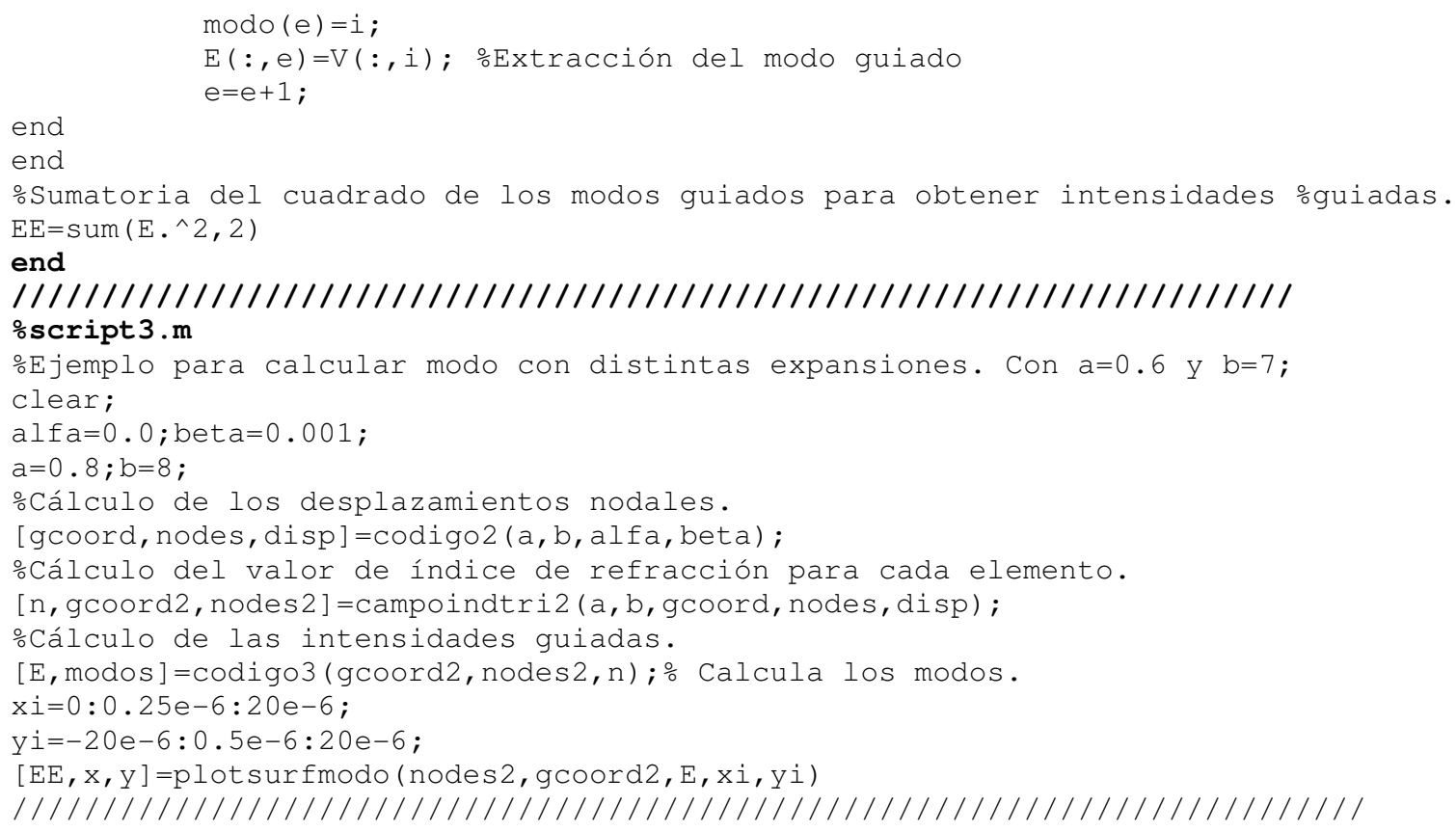





\section{Bibliografía}

[A81]E. Anastassakis, Inelastic light scattering in the presence of uniaxial stresses, J. Raman Spectrosc., 10, 64 (1981). [ABQM]Manual de software comercial ABAQUS. http://abaqus.me.chalmers.se/v6.8/books/usb/default.htm, [ANI74]S.I.Anisimov,B.L.Kapeliovich,T.L.Perelman, Electron-emission from surface metals induced by ultrashort laser pulses.Zh.Eksp.Teor. Fiz. 66,776 (1974).

[BA08] S. Basu, A. Zhou and M. W. Barsoum, Reversible dislocation motion under contact loading in LiNbO3 single crystal, J. Mater. Res. 23, 1334(2008).

[BA96]K.J. Bathe, FiniteElement Procedures,Prentice Hall,U.S.A., 1996.

[BELL06]Y.Bellouard, T.Colomb, C.Depeursinge, M.Dugan, A.A.Said, P.Bado, Nanoindentation and birefringence measurements on fused silica specimen exposed to low-energy femtosecond pulses, Opt. Express 14, 8360 (2006).

[BEN09]A.Benayas, D.Jaque, B.McMillen, K.P.Chen. High repetition rate UV ultrafast laser inscription of buried channel waveguides in Sapphire: fabrication and fluorescence imaging via ruby R lines, Opt. Express 17, 10076 (2009).

[BER09]S.Berweger and M.B. Raschke, Polar phonon mode selection rules in tip-enhanced Raman scattering. J. Raman Spectrosc., 40, 1413(2009).

[BHAR05]V. R. Bhardwaj, E. Simova, P. B. Corkum, D.M.Rayner, C. Hnatovsky, R. S. Taylor,B.Schreder, M. Kluge, and J. Zimmer, Femtosecond laser-induced refractive index modification in multicomponent glasses, J. of Appl. Phys. 97, $083102(2005)$

[BHA05]S.Bhagavat and I. Kao, Nanoindentation of lithium niobate: hardness anisotropy and pop-in phenomenon, Mat. Sci. and Eng. A 393, 327,(2005).

(http://www.sciencedirect.com/science/article/pii/S0921509304013462)

[BIA13]D. Biasetti, E. Neyra, J. R. Vázquez de Aldana, L. Roso and G. A. Torchia, Buried waveguides in Nd:YLF crystals obtained by femtosecond laser writing under double line approach, Appl. Phys. A, 110, March 2013, 595 (2013).

[BO03]R. W. Boyd, Nonlinear Optics (Elsiever Science, USA, 2003).

[BO12] A.Borz, V.Schulz,Computational Optimization of Systems Governed by Partial Differential Equations, edited by SIAM, 2012.

[BRI76]R. J. Briggs and A. K. Ramdas, Piezospectroscopic study of the Raman spectrum of cadmium sulfide, Phys. Rev. B 13, 5518(1976).

[BRES11]Lena Bressel, D.Ligny, C. Sonneville, V. Martinez, V. Mizeikis, R.Buividas and S. Juodkazis, Femtosecond laser induced density changes in $\mathrm{GeO} 2$ and $\mathrm{SiO} 2$ glasses: fictive temperature effect, Opt. Mat. Express 1, 605 (2011).

[BUL84]C.H.Bulmer, Characteristics of Ti-indiffused waveguides in MgO-doped LiNbO3, Electron. Lett. 20, 902 (1984).

[BUR07]J.Burgoff, S. Nolte, A. Tünnermann, Origins of waveguiding in femtosecond laser-structured LiNbO3, Appl. Phys. A 89, 127 (2007).

[BURHA07]J. Burgoff, H. Hartung, S. Nolte, A. Tünnerman, Structural properties of femtosecond laser-induced modifications in LiNbO3, Appl. Phys. A 86, 165 (2007).

[BURGRE07]J. Burghoff, C. Grebing, S. Nolte, and A. Tunnermann, Waveguides in lithium niobate fabricated by focused ultrashort laser pulses, Appl. Surf. Sci.253, 7899 (2007).

[CHA07]E. W. V. Chaves, Mecánica de medios continuos: conceptos básicos, (Centro Internacional de Métodos Numéricos en Ingeniería (CIMNE), Barcelona, 2007).

[DA96]K. M. Davis, K. Miura, N. Sugimoto, and K. Hirao, Writing waveguides in glass with a femtosecond laser, Opt. Lett. 21, 1729 (1996).

[FERR05]P. Ferraro, L.Aiello, S. De Nicola, A.Finizio, R. Osellame, N. Chiodo, V. Maselli, G. Cerullo and P. Laporta, Interferometric method for measuring the refractive index profile of optical waveguides directly written in glass substrates by femtosecond laser, Nano- and Micro-Metrology, edited by Heidi Ottevaere, Peter DeWolf, Diederik S. Wiersma, Proceedings of SPIE Vol. 5858 (SPIE, Bellingham, WA, 2005).

[FI07]A. C. Fischer-Cripps, Introduction to Contact Mechanics, Mechanical Engeniering Series, Springer, Australia, 2007. 
[FI84]M. Filho, V. Lemos, F. Cerdeira, Pressure dependence of LiNbO3 and LiTaO3, J. Raman Spectrosc. 15, 367 (1984).

[FY88]G.W. Fynn, G. W. Fynn, W.J.A. Powell, Cuting and polishing optical and electronic materials, A. Hilger, 1988.

[FLE86]G.R.Fleming, 1986, Chemical Applications of Ultrafast Spectroscopy, (Oxford University Pres, New York).

[GA06]R. Gattas, Femtosecond-laser interactions with transparent materials: applications in micromachining and supercontinuum generation, $\mathrm{PhD}$ thesis, Harvard University, Cambridge, Massachusetts, 2006.

[GA12]E. Gamaly, Femtosecond Laser-matter Interactions: Theory, Experiments and Applications. (Contemporary Physics, Volume 53, Issue 6, 2012).

[GIB82]L.J. Gibson and M.F. Ashby, The Mechanics of Three-Dimensional Cellular Materials, Proc. R. Soc. Lond. A. 382, 43 (1982).

[HE82]H. R. Hertz, Ueber die Beruehrung elastischer Koerper (On Contact Between Elastic Bodies), in Gesammelte Werke (Collected Works), Vol. 1, Leipzig, Germany, 1895.

[HEN09]O.Henneberg, R.Menzel, R.Elsner, D. Korn, J. Siebenmorgen, and G. Huber, X-Ray Diffraction Measurements of a Femtosecond-Laser Written Waveguides in an YAG Single Crystal, The European Conference on Lasers and Electro-Optics,OSA, Germany, CM_P2 (2009).

[HO]Matti Hotokka,Lecture Notes, XI.Raman Spectroscopy, Department of Physical Chemistry, Abo Akademi University, http://users.abo.fi/mhotokka/mhotokka/lecturenotes/ms04.d/ms04-raman-eng.pdf.

[JA85]Jackel, J.L., Proton exchange in MgO-doped LiNbO3, Electron. Lett. 21, 509 (1985).

[JAY83]A. Jayamaran. Diamond anvil cell and high-pressure physical investigations. Rev. Mod. Phys. 55, 65 (1983).

[JAY86]A. Jayaraman and A. A. Ballman, Effect of pressure on the Raman modes of LiNbO3 and LiTaO3, J. of App. Phys. 60, 1208 (1986).

[KIM97] S.C. Kim, B.M. Jin, J. Kim, Study of MgO-substitution in LiNbO3 single-crystals by using micro-probe Raman-Spectroscopy, J. Korean Phys. Soc. 31, 689(1997).

[KON10]P.Kongsuwan, S.Vukelic, Y.L.Yao and H. Wang, Characterization of Morphology and Mechanical Properties

of Glass Interior Irradiated by Femtosecond Laser, J. Manuf. Sci. Eng. 132, 041009(2010).

[KWON97] Y.Kwon and H.Bang, The Finite Element Method using MATLAB, C.R.C., U.S.A, 1997.

[LA] http://www.lambdaphoto.co.uk/pdfs/Inrad_datasheet_LNB.pdf

[LAB01] http://www.horiba.com/scientific/products/raman-spectroscopy/software/functionality/

[LAB02]http://www.newport.com/images/webDocuments-EN/images/12126.PDF

[LAB03] https://www.horiba.com/fileadmin/uploads/Scientific/Documents/Raman/labrambro.pdf

[LED04]H.Ledbetter a, H. Ogi, N. Nakamura, Elastic, anelastic, piezoelectric coefficients of monocrystal lithium niobate, Mechanics of Materials 36, 941 (2004).

[LEN07]K. Lengyel, L. Koacs, A. Peter, K. Polgar, G. Corradi, P. Bourson, The effect of Mg doping on the Raman spectra of LiNbO3 crystals. Phys. Stat. Sol (c) 4, 847 (2007).

[LIN03]G. Lifante, Integrated Photonics: Fundamentals (John Wiley \& Sons, London, 2003).

[MA13]C.K. Maiti, T.K. Maiti, Strain-Engineered MOSFETs, CRC Press, 2013.

[MAR86]Martinez, O. E., Grating and prism compressor in the case of finite beam size, JOSA B 3, 929 (1986).

[MI06]H. Misawa and S. Juodkazis, 3D Laser Microfabrication: Principles and Applications (Wiley-VCH, Weinheim, 2006).

[MO11]Tesis doctoral de D.F. Moreau, Conformación de haces láser de pulsos ultracortos para la optimización de la escritura de guias de onda en dieléctricos, Universidad Autónoma de Madrid, 2011.

[MOU00]R. Mouras, M.D. Fontana, P. Bourson, A. V. Postnikov, Lattice site of Mg ion in LiNbO 3 crystal determined by Raman spectroscopy. J. Phys. Condens. Matter 12, 5053(2000).

[MY09]B.G. Mytsyk, S. Anatoliy, S. Andrushchak, N. M. Demyanyshyn,Y. P. Kost, A. V. Kityk, P. Mandracci and W. Schranz, Piezo-optic coefficients of Mgo-doped LiNbO3 crystals, App. Opt. 48, 1904 (2009).

[NOBUR04] S. Nolte, J. Burghoff, M. Will, and A. Tünnermann, Femtosecond writing of high quality waveguides inside phosphate glasses and crystalline media using a bifocal approach, Commercial and Biomedical Applications of Ultrafast Lasers IV Proceedings, SPIE 5340, 1640, CA(USA), (2004).

[OBER98] P. Oberson, B. Gisin, B. Huttner and N. Gisin, Refracted near-field measurements of refractive index and geometry of silica-on-silicon integrated optical waveguides, Appl. Opt. 37, 7268 (1998).

[OKA05] K. Okamoto, Fundamentals of Optical Waveguides, Elsiever, U.S.A, 2005.

[OLI04]W. C. Oliver, G. M. Pharr, Measurement of hardness and elastic modulus by instrumented indentation: Advances in understanding and refinements to methodology, Journal of Materials Research, 19, 3 (2004).

[OSE12]R. Osellame, G. Cerullo, R. Ramponi, Femtoseond laser micromachining (Springer-Verlag, Berlin, 2012).

[PA06]M.N. Palatnikov, I.V. Biryukova, N.V. Sidorov, A.V. Denisov, V.T. Kalinnikov, P.G.R. Smith, V.Ya. Shur, Growth and concentration dependencies of rare-earth doped lithium niobate single crystals, J. of Crystal Growth 291 390 (2006). 
[PE13]G.Pezzotti, H. Hagihara, W. Zhu, Quantitative investigation of Raman selection rules and validation of the secular equation for trigonal LiNbO3, J. Phys. D: Appl. Phys. 46, 145103 (2013).

[PER04]P.O.Persson, G. Strang, A Simple Mesh Generator in MATLAB. SIAM Review, 46, pp. 329, 2004.

[POU03]B. Poumellec, L. Sudrie, M. Franco, B. Prade, A. Mysyrowicz, Femtosecond laser irradiation stress induced in pure sílica, Opt. Express 11, 1070 (2003).

[QIN01]L. Qin, K.L. Teo, Z.X. Shen, C.S. Peng, J.M. Zhou, Raman scattering of Ge/Si dot superlattices under hydrostatic pressure, Phys. Rev. B 64 (2001) 075312.

[QUI09]R.Quispe-Siccha, E.V. Mejía-Uriarte, M. Villagrán-Muniz, D. Jaque, J. García Solé, F. Jaque, R. Y. Sato-Berru, E. Camarillo, J. Hernández, H. Murrieta, The effect of $\mathrm{Nd}$ and $\mathrm{Mg}$ doping on the micro-Raman spectra of LiNbO3 singlecrystals. J. Phys.: Condens. Matter 21, 145401(2009).

[RA01]P. Rai-Choudhury. MEMS \& MOEMS: technology and applications, SPIE Ed.USA (2001).

[RI04]M.Richardson, A.Zoubir, C.Rivero, C. Lopez, L.Petit, and K.Richardson, Femtosecond laser micro-structuring and refractive index modification applied to laser and photonic devices, Micromachining Technology for Micro-Optics and Nano-Optics II, 5347 (2004).

[RO08]A. Ródenas, A.H.Nejadmalayeri, D.Jaque, P. Herman. Confocal Raman imaging of optical waveguides in LiNbO3 fabricated by ultrafast high-repetition rate laser-writing, Opt Express. 16(18):13979 (2008).

[RO09] A. Ródenas, L. M. Maestro, M. O. Ramírez, G. A. Torchia, L. Rosso, F. Chen, and D. Jaque, Anisotropic lattice changes in femtosecond laser inscribed Nd3+:MgO:LiNbO3 optical waveguides, J. Appl. Phys. 106, 013110 (2009).

[RO09_2]A. Ródreducitenas, Direct femtosecond laser writing of $3 d$ photonic structures in rare-earth doped lithium niobate, Tesis doctoral, Universidad Autónoma de Madrid, 2009.

[RYU12]S.Ryu, Q.Zhao, M.Hecker, H.Son, K.Byun, Micro-Raman spectroscopy and analysis of near-surface stresses in silicon around through-silicon vias for three-dimensional interconnects, J. Appl. Phys. 111, 063513 (2012);

[SA13]S.Sanna, A. Riefer, S. Neufeld, W. G. Schimdt, G. Berth, M. Rüsing, A. Widhalm, and A. Zrenner, Vibrational Fingerprints of LiNbO3-LitaO3 Mixed Crystals, Ferroelectrics, 447, 63 (2013).

[SAN]J.C. del C. Sánchez, Elasticidad, Escuela Técnica Superior de Ingenieros Industriales de Valladolid. http://www.eis.uva.es/reic/Elas Web/directorio.htm

[SCHA01]C.B.Schaffer, Interaction of femtosecond laser pulses with transparent materials, Ph.D. thesis. Harvard University, Cambridge, Massachusetts, 2001.

[STRI85]D.Strickland, G. Mourou. Compression of amplified chirped optical pulses. Opt. Commun. 56, 219 (1985).

[SVE11]V. Sverdlov, Strain-Induced Effects in Advanced MOSFETs, Springer-Verlag Wien, Germany (2011).

[SZA06]A.Szameit, J.Burghoff, T.Pertsch, S.Nolte and A. Tünnermann, Two-dimensional soliton in cubic fs laser written waveguide arrays in fused silica, Opt. Express 14, 6055 (2006).

[TAY03] R. S. Taylor, C. Hnatovsky, E. Simova, D. M. Rayner, M. Mehandale, V. R. Bhardwaj and P. B. Corkum, Ultra-high resolution index of refraction profiles of femtosecond laser modified silica structures, Opt. Express 11, 775 (2003).

[TE71]V.J. Tekippe, A.K. Ramdas, A piezospectroscopic study of the Raman spectrum of $\alpha$ - quartz". Physics Letters A, 35, 143(1971).

[TE73]V. J. Tekippe, A. K. Ramdas, Sergio Rodriguez, Piezospectroscopic Study of the Raman Spectrum of alphaQuartz, Phys. Rev. B, 8, 706 (1973).

[TI96]TCT Ting, Anisotropic elasticity: Theory and applications, Oxford University, U.S.A, 1996.

[TO14]X.C.Tong, Advanced Materials for Integrated Optical waveguides, Springer Series in Advanced Microelectronics 46(2014).

[VA01]Tesis doctoral de C. Vasile, Ab-initio lattice dynamics in LiNbO3 and LiTaO3, University of Osnabrück, 2001.

[VU08]S.Vukelic, B.Gao, S. Ryu, Y. L.Yao, Structural modification of amorphous fused silica under femtosecond laser irradiation. Procedings of the 2008 International Manufacturing Science and Engineering Conference. MSEC2008, Illinois, USA,2008.

[VO10]W.Voigt, Lehrbuch der Kristallphysik, Lipzig, 560 (1910).

[WILL05]M. Will, J. Burghoff, S. Nolte, and A. Tünnermann, Detailed investigations on femtosecond induced modifications in crystalline quartz for integrated optical applications, in Commercial and Biomedical Applications of Ultrafast Lasers IV Proceedings, edited by J. Neev, C. B. Schaffer, A. Ostendorf,and S. Nolte (SPIE, 2005), Vol. 5714, pp. 261-270.

[WO02]K.K.Wong, Properties of lithium niobate, published by INSPECT, London, United Kingdom (2002).

[WO96]L. De Wolf, Micro-Raman spectroscopy to study local mechanical stress in silicon integrated circuits, Semicond. Sci. Technol. 11, 139(1996).

[YA08]F.Yang and J.C.M.Li, Micro and Nano Mechanical Testing of Materials and Devices, Springer, USA, 2008.

[YAN08] W. Yang, Femtosecond Laser Writing in Transparent Material, Ph.D. Thesis, University of Southampton, Southampton, 2008. 